\title{
The Role of the E3 Ubiquitin Ligase Cdh1-APC in Axon Growth in the Mammalian Brain
}

\author{
Dissertation \\ for the award of the degree \\ "Doctor rerum naturalium" \\ of the Georg-August-Universität Göttingen \\ Submitted by

\section{Madhuvanthi Kannan} \\ Born in Chennai, India
}

Göttingen, July 2012

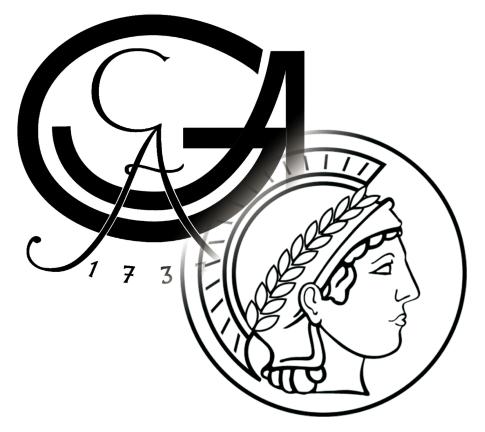


Member of the Thesis Committee (Reviewer): Dr. Judith Stegmüller Molecular and Cellular Neurobiology, Max Planck Institute of Experimental Medicine, Göttingen

Member of the Thesis Committee (Reviewer): Dr. Oliver Schlüter Molecular Neurobiology,

European Neuroscience Institute, Göttingen

Member of the Thesis Committee: Prof. Dr. Gregor Bücher Blumenbach Institute, Department of Developmental Biology, Georg-August-Universität, Göttingen

Date of Oral Examination: August 22, 2012 
I hereby confirm that this dissertation, "The Role of the E3 Ubiquitin Ligase Cdh1-APC in Axon Growth in the Mammalian Brain", was prepared on my own and with no other sources or aids than quoted. 


\section{Acknowledgements}

"The vision of the leader is the hope of the pack"-Anonymous. True to this, my supervisor Dr. Judith Stegmüller has been the sole drive to my finish line. Not just a brainbehind but Judith has also been a great mentor, a huge source of inspiration and the kind of leader who will bank on you during rough tides and help you get forward. I am greatly indebted to her for all that she has taught me and for four years of incessant support in the lab.

I am very grateful to my thesis committee members Prof. Gregor Bücher and Dr. Oliver Schlüter for their comments and criticisms that honed my study. I am also thankful to the members of my extended committee Prof. Andreas Wodarz, Prof. Anastassia Stoykova and Dr. Till Marquardt for consenting to evaluate my thesis and defense.

I thank the GGNB scientific coordinator Prof. Michael Hörner for his valuable pointers and the GGNB staff for help with administrative work.

I wish to thank our collaborator Prof. Takanobu Nakazawa, University of Tokyo, Japan for sharing his reagents for the p250GAP project and Dr. Miso Mitkovski, MPI-EM for helping me with confocal microscopy and imaging. The animal facility at MPI-EM needs a special mention for taking care of our Cdh1 colony and I wish to thank them for prompt emails. Thanks go to all the non-scientific staff of MPI-EM for making my life easy at work.

Thanks to Jan Höber and Diana Urrego Blanco for contributing to this work during their rotations. Huge thanks to Nicola, Mayur, Annika, Anna, Siv, David and Chaitali for making my $\mathrm{PhD}$ a very pleasant and memorable experience. Big thanks to Shih-Ju who has been a great 'buddy' and with whom I've shared several indelible moments over coffee and at the gym.

Getting here would have been very hard but for the continued support of Ganesh. He has been a great push during the tough times and has made me believe in myself every time I stumbled. I am grateful to him for being there.

Behind the success of every person there's a secret. My secret is the boundless support of Amma, Appa and Paati. Indeed, there's no success without the three of them and I hope to make them proud someday. I just cannot thank them enough! 


\section{Abbreviations}

AA amino acid

AMPA alpha-amino-3-hydroxy-5-methyl-4-isoxazole-propionic acid

ANOVA analysis of variance

APC anaphase promoting complex

APS ammonium peroxydisulphate

ATP adenosine 5'-triphosphate

BDNF brain derived neurotrophic factor

bHLH basic helix-loop-helix

BME basal medium eagle

BMP bone morphogenetic protein

BSA bovine serum albumin

${ }^{\mathbf{0}} \mathbf{C} \quad$ degree Centigrade/Celsius

CA constitutively active

cAMP 3'-5' cyclic adenosine monophosphate

Cdc20 cell division cycle protein 20

Cdc42 cell division cycle protein 42

Cdh1 cell division cycle 20 homolog 1

cDNA complementary deoxyribonucleic acid

CNS central nervous system

CREB 3'-5' cyclic adenosine monophosphate response element binding protein

CSPG chondroitin sulphate proteoglycan

$\mathbf{C}(\mathbf{t}) \quad$ threshold cycle

DBM destruction box mutant

D-box destruction box

DIV day in vitro

DMEM Dulbecco's modified eagle medium

DN dominant negative

DNA deoxyribonucleic acid

DRG dorsal root ganglion

DTT dithiothreitol

E embryonic day

EDTA Ethylenediaminetetraacetic acid 


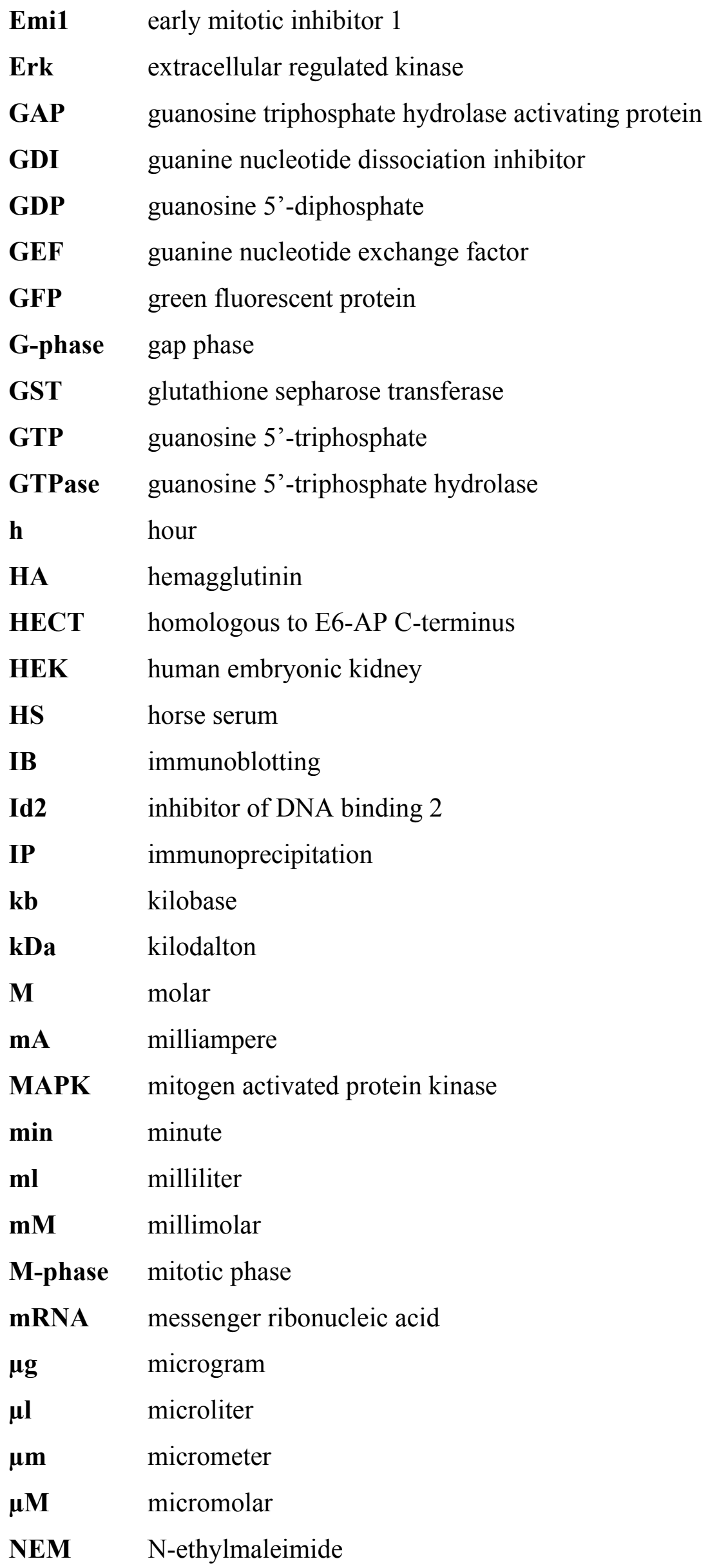




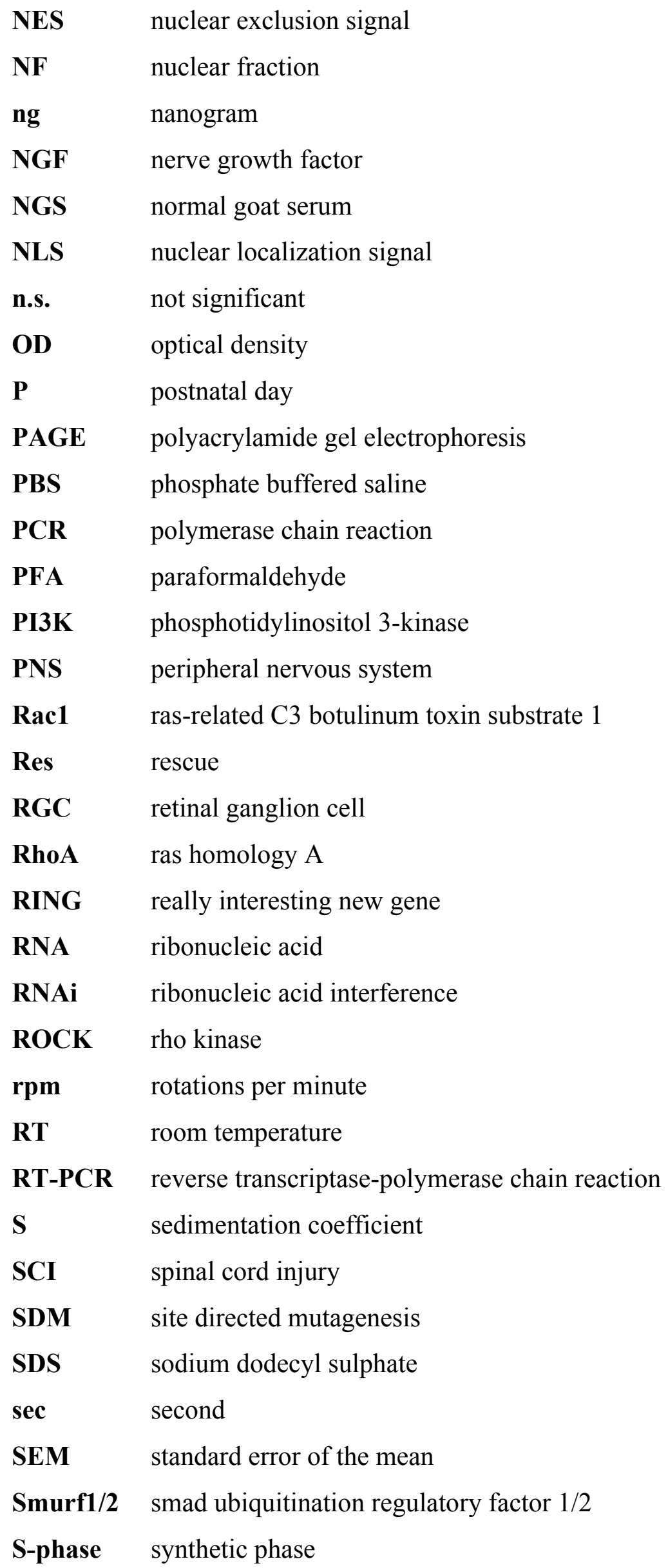


TEMED tetramethylethylenediamine

TPR tetratricopeptide repeat

Trk tropomyosin kinase

U unit

$\mathbf{v} / \mathbf{v} \quad$ volume-to-volume

W week

WD Wallerian degeneration

WT wild-type

w/v weight-to-volume 


\section{$\underline{\text { Nucleotide }}$ Single letter code}

$\begin{array}{ll}\text { Adenine } & \text { A } \\ \text { Guanine } & \text { G } \\ \text { Cytosine } & \text { C } \\ \text { Thymine } & \text { T } \\ \text { Uracil } & \text { U }\end{array}$

\begin{tabular}{|c|c|c|}
\hline Amino Acid & Abbreviation & Single letter code \\
\hline Alanine & Ala & $\mathrm{A}$ \\
\hline Arginine & Arg & $\mathrm{R}$ \\
\hline Asparagine & Asn & $\mathrm{N}$ \\
\hline Aspartic acid & Asp & $\mathrm{D}$ \\
\hline Cysteine & Cys & $\mathrm{C}$ \\
\hline Glutamic acid & Glu & $\mathrm{E}$ \\
\hline Glutamine & Gln & Q \\
\hline Glycine & Gly & $\mathrm{G}$ \\
\hline Histidine & His & $\mathrm{H}$ \\
\hline Isoleucine & Ile & I \\
\hline Leucine & Leu & $\mathrm{L}$ \\
\hline Lysine & Lys & $\mathrm{K}$ \\
\hline Methionine & Met & M \\
\hline Phenylalanine & Phe & $\mathrm{F}$ \\
\hline Proline & Pro & $\mathrm{P}$ \\
\hline Serine & Ser & S \\
\hline Threonine & Thr & $\mathrm{T}$ \\
\hline Tryptophan & $\operatorname{Trp}$ & $\mathrm{W}$ \\
\hline Tyrosine & Tyr & $\mathrm{Y}$ \\
\hline Valine & Val & $\mathrm{V}$ \\
\hline Unspecified & & $X$ \\
\hline
\end{tabular}




\section{Contents}

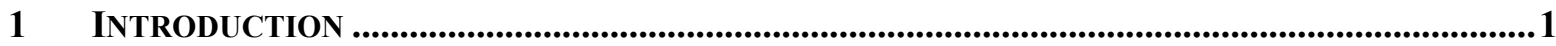

1.1 Extrinsic pathways of axonal morphogenesis and axon regeneration ..................................... 1

1.1.1 Wiring the brain: Extrinsic cascades in axon growth ................................................ 1

1.1.2 Fetters in axon regeneration: Glial inhibition of CNS regeneration ................................

1.2 Ubiquitination as a means of cell-intrinsic control of neuronal morphogenesis and axon

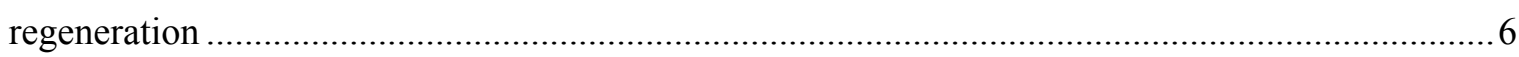

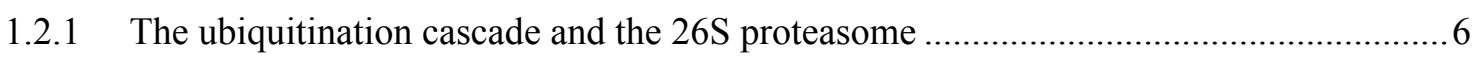

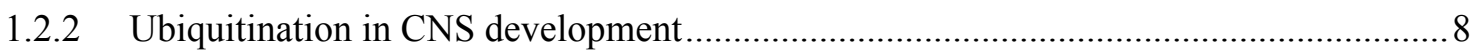

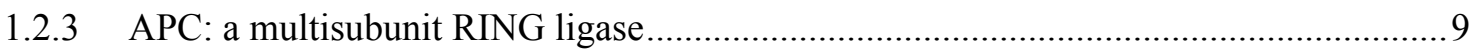

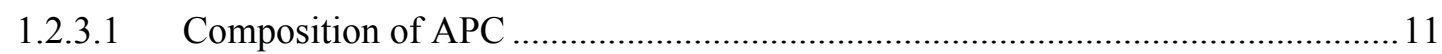

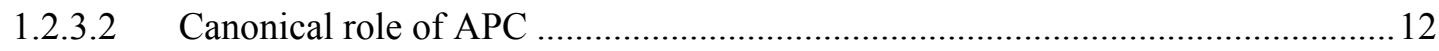

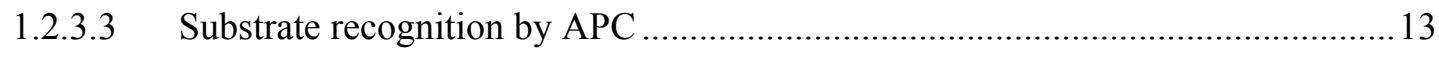

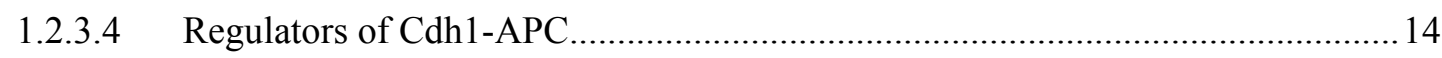

1.2.3.5 Cdh1-APC: Emerging roles in neuronal development and regeneration ................14

1.3 Overcoming extrinsic inhibition by manipulating intrinsic growth capacity ......................... 17

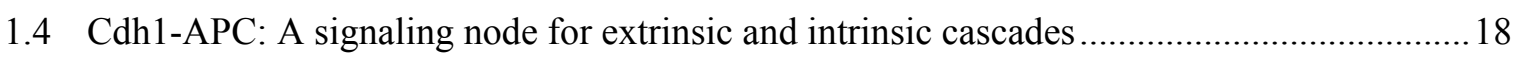

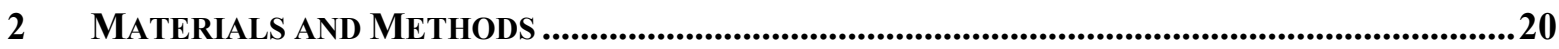

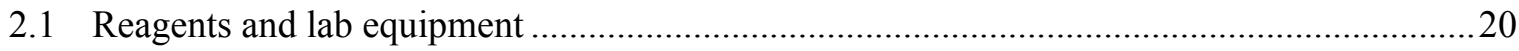

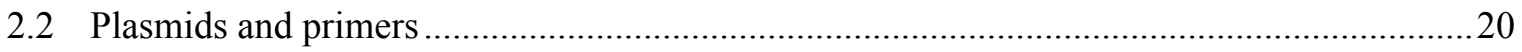

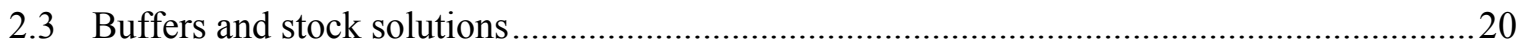

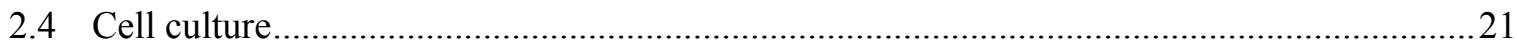

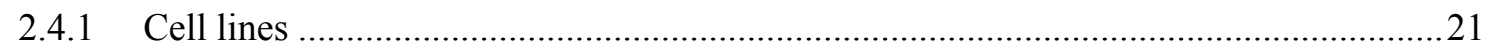

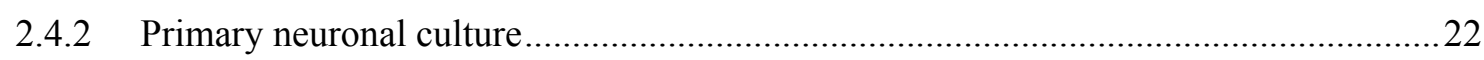

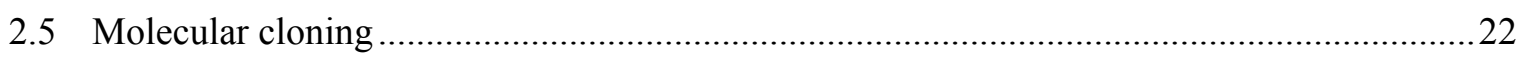

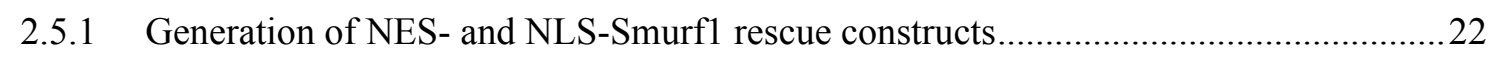

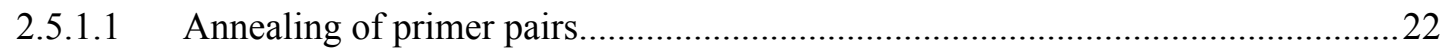

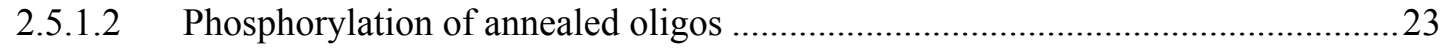

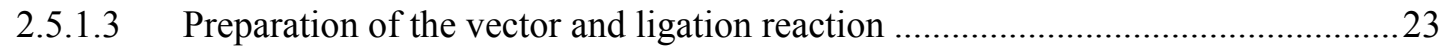

2.5.1.4 Bacterial transformation and selection of positive clones.....................................23

2.5.2 Generation of Myc-ubiquitin-p250GAP and Myc-p250GAP-ubiquitin fusion constructs 24

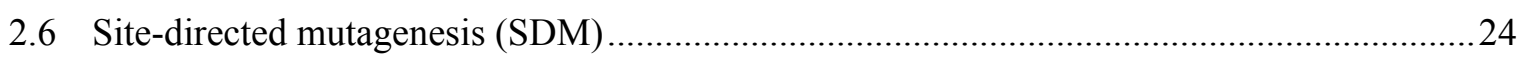

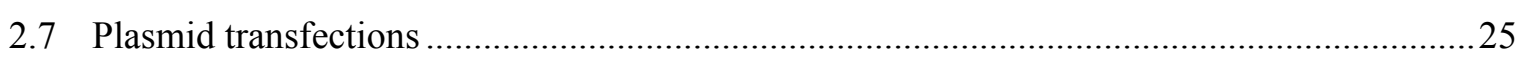

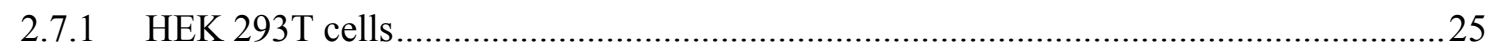




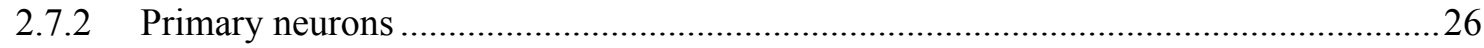

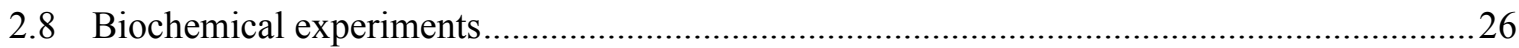

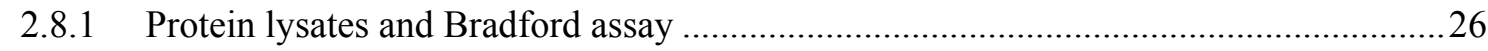

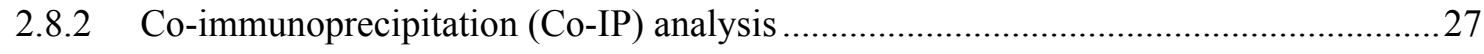

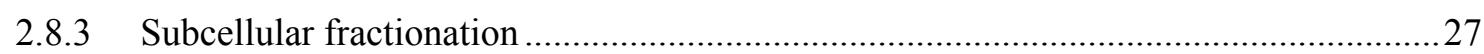

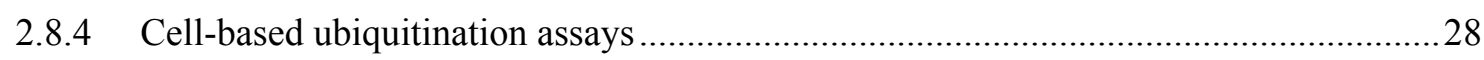

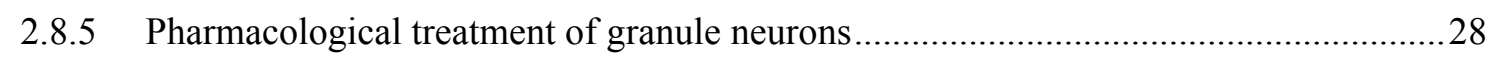

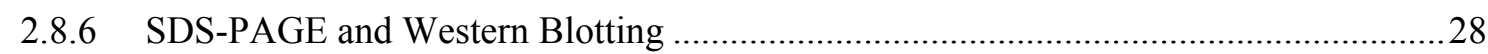

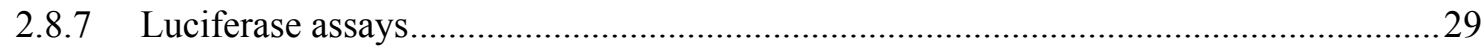

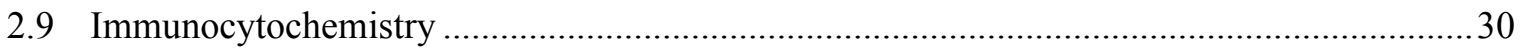

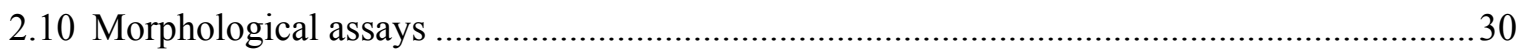

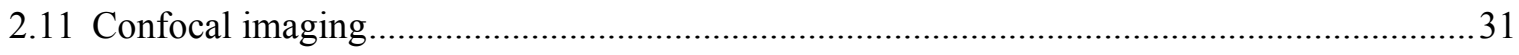

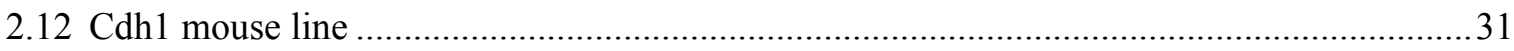

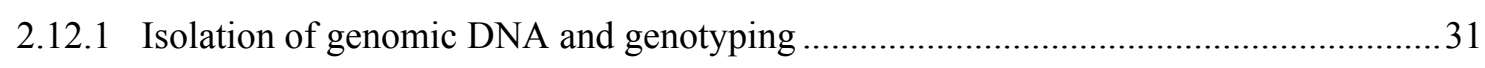

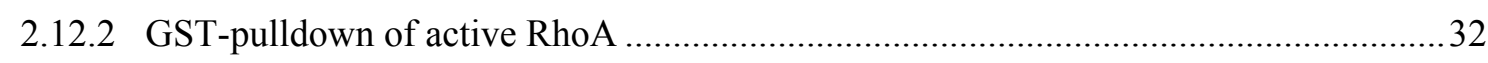

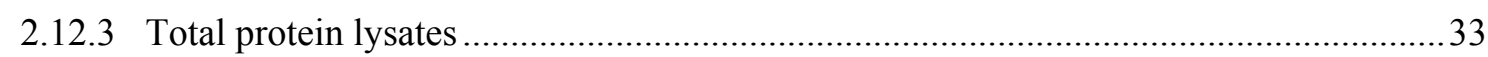

2.12.4 RNA isolation, cDNA synthesis and quantitative RT-PCR ............................................33

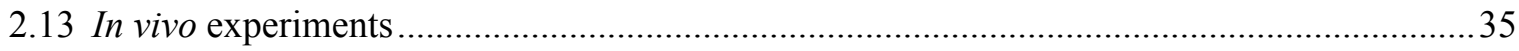

2.13.1 In vivo electroporation of cerebella of postnatal rat pups ...............................................

2.13.2 Isolation of cerebella, fixation and immunohistochemistry on cryo-sections ................35

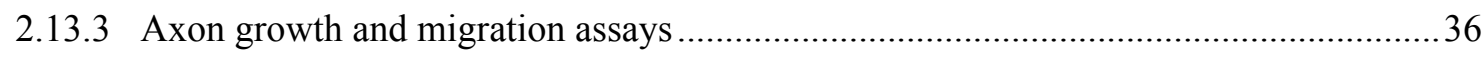

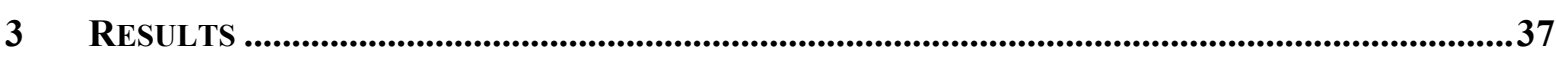

3.1 Growth-promoting signaling programs antagonize Cdh1-APC-inhibition of axon growth ...37

3.1.1 Inhibition of PI3-kinase signaling inhibits Cdh1 knockdown-induced axonal growth in vitro

3.1.2 Inhibition of MAP-kinase signaling significantly reduces axon length in Cdh1 knockdown neurons .39

3.2 Cdh1-APC exerts control on axon growth by stimulating growth-inhibitory cytoskeletal Rho

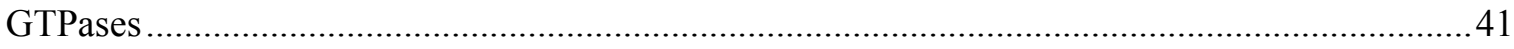

3.2.1 RhoA activity reverses Cdh1 knockdown-stimulated axon growth...............................41

3.2.2 Interfering with Rac1 activity impairs axon length in Cdh1 knockdown neurons ........42

3.2.3 Active Cdc42 significantly reduces axon length under Cdh1 knockdown conditions ..43

3.3 Cdh1 interacts with Smurf1, a HECT ubiquitin ligase that degrades RhoA, in the control of axon growth....

3.3.1 Smurf1, a brain-abundant HECT ligase, is a novel interactor of Cdh1 .44

3.3.2 Smurfl is degraded in a proteasome-dependent manner and its protein levels are induced in Cdh1-heterozygotes 
3.3.3 Smurf1 is poly-ubiquitinated and targeted for degradation by Cdh1-APC .48

3.3.4 Two D-boxes close to its C-terminus mediate the interaction of Smurf1 with Cdh1 ....50

3.3.5 Smurf1 double D-box mutant exhibits greater stability and reduced affinity for Cdh1 as compared to the wild-type protein 51

3.3.6 Smurfl enhances axon growth independently of the neuronal cell type 53

3.3.7 Smurfl acts downstream of Cdh1 in a linear pathway in the control of axon growth...57

3.3.8 The double D-box mutant of Smurf1 exhibits a gain-of-function in the regulation of axon growth.

3.3.9 Smurf1 promotes axon growth downstream of Cdh1-APC by degrading RhoA ..........59

3.3.10 Smurf1 DBM3/4 protects neurons from the growth inhibitory effects of myelin 62

3.4 p250GAP, a Rho GTPase activating protein, promotes axon growth downstream of Cdh1-

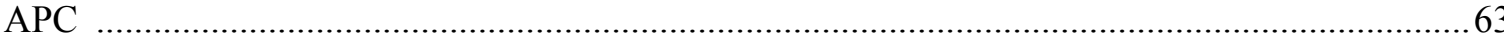

3.4.1 p250GAP, a brain-enriched RhoGAP is a novel interactor of Cdh1 …........................63

3.4.2 Knockdown of p250GAP reduces axon growth in cerebellar granule neurons ..............65

3.4.3 p250GAP is not degraded in a proteasome-dependent manner and its protein levels do not accumulate in the Cdh1-heterozygotes

3.4.4 p250GAP undergoes non-proteolytic ubiquitination at its C-terminus ..........................68

3.4.5 C-terminally ubiquitinated p250GAP is stable but fails to promote axon growth.........70

3.4.6 p250GAP regulates axon growth downstream of Cdh1-APC ......................................71

3.4.7 p250GAP acts in shared pathway together with Smurf1 in axon growth regulation.....72

3.5 p250GAP and Smurf1 control axon growth and migration in the developing cerebellum in vivo 74

3.5.1 Knockdown of p250GAP or Smurf1 in the developing cerebellum causes a dramatic failure of granule neurons to migrate to the internal granule layer.....

3.5.2 p250GAP or Smurf1 knockdown distorts the development and elongation of granule neuron parallel fibers in the early postnatal rat cerebellum .76

4 DISCUSSION .78

4.1 MAPK and PI3K signaling antagonize Cdh1-inhibition of axon growth...............................78

4.2 Cdh1-APC controls axon growth by modulating cytoskeletal proteins .................................79

4.3 E3 ligase Smurf1: a novel Cdh1 substrate that potentiates axon growth................................ 81

4.4 Smurf1-degradation of RhoA is central to Cdh1-APC/Smurf1 pathway .................................8 84

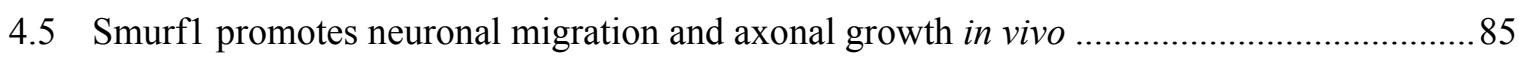

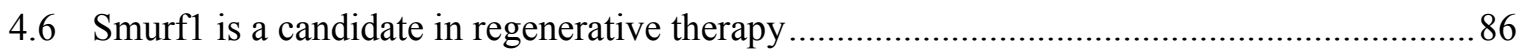

4.7 The Cdh1-APC/Smurf1/RhoA pathway: scope for further research .......................................87

4.8 GAPs are candidate mediators of Cdh1-dependent RhoA regulation ...................................... 89

4.9 Growth-promoting p250GAP undergoes activity-modifying ubiquitination .........................90

4.10 p250GAP is indispensible for neuronal development in vivo................................................91 
4.11 Smurf1 and p250GAP: a potential cooperative control of RhoA .........................................91

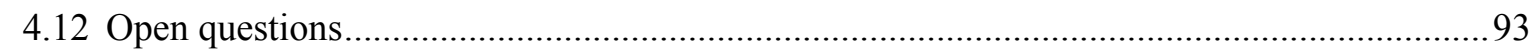

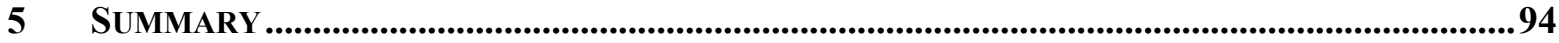

6 REFERENCES

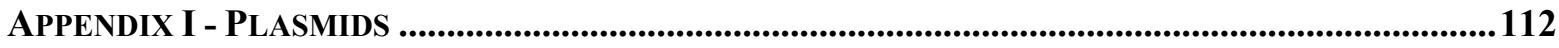

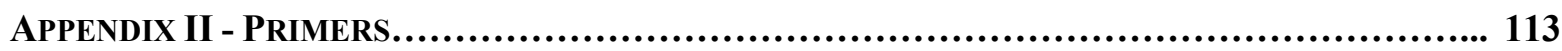

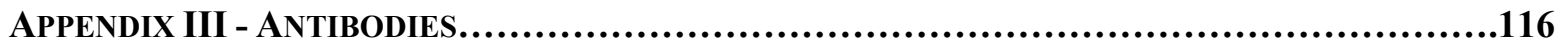

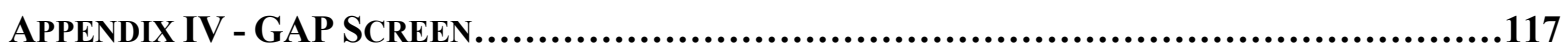

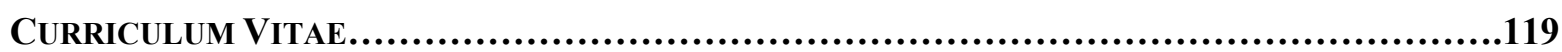




\section{Introduction}

A fascinating aspect of the human brain is the high order of connectivity arising from the thousands of synapses of each of one hundred billion neurons. Despite such complexity, precise wiring is achieved by an ordered cascade of molecular events that controls phenomena ranging from neuritogenesis to neuronal differentiation. Axons, the presynaptic processes of a neuron carry all of the vital information for the brain's processing and their role is underscored by their varied morphology. Studying the molecular control of axonal morphogenesis thus becomes a requisite to decoding the structure and function of the brain.

\subsection{Extrinsic pathways of axonal morphogenesis and axon regeneration}

Axonal morphogenesis depends on an intricate orchestration of extrinsic and intrinsic events. Differentiation of a neuron begins with the development of fine F-actin protrusions called 'filopodia' which engorge microtubules to define a discrete neuronal structure called the "axon growth cone" (Cajal, 1890; Goldberg and Burmeister, 1986). The growth cone is a highly motile sensor, which is steered in response to guidance cues and trophic factors in its environment and eventually finds its postsynaptic target. Interstitial axon branching and axon pruning, followed by dendritic development also under the surveillance of extrinsic factors, further ensure precise neuronal connectivity (Kalil et al., 2000; Low and Cheng, 2006).

As with axonal development, axon regeneration following central nervous system (CNS) injury is also heavily influenced by the extracellular environment. Notably myelin, rich in growth inhibitory proteins, has been extensively studied as an extrinsic suppressor of axon growth and regeneration (McKerracher et al., 1994; Mukhopadhyay et al., 1994; Schwab, 1993).

This section discusses the major extrinsic players that modulate axonal growth and regeneration in the CNS.

\subsubsection{Wiring the brain: Extrinsic cascades in axon growth}

The discovery of the neurotrophins, nerve growth factor (NGF), brain-derived neurotrophic factor (BDNF), neurotrophin 3 (NT3) and neurotrophin 4 (NT4) (Barde et al., 1982; Hallbook et al., 1991; Levi-Montalcini and Hamburger, 1951; Maisonpierre et al., 1990) paved way to a better understanding of the secreted factors that support neuronal growth and survival. 
Neurotrophins mediate their effects by binding to transmembrane receptors, the tropomyosin kinases (Trks) (Huang and Reichardt, 2001; Kaplan et al., 1991a; Klein et al., 1991). A variety of adaptors bind activated Trks allowing trophic signaling to be integrated with several intracellular cascades, which contribute to the biological functions elicited by neurotrophins (MacDonald et al., 2000).

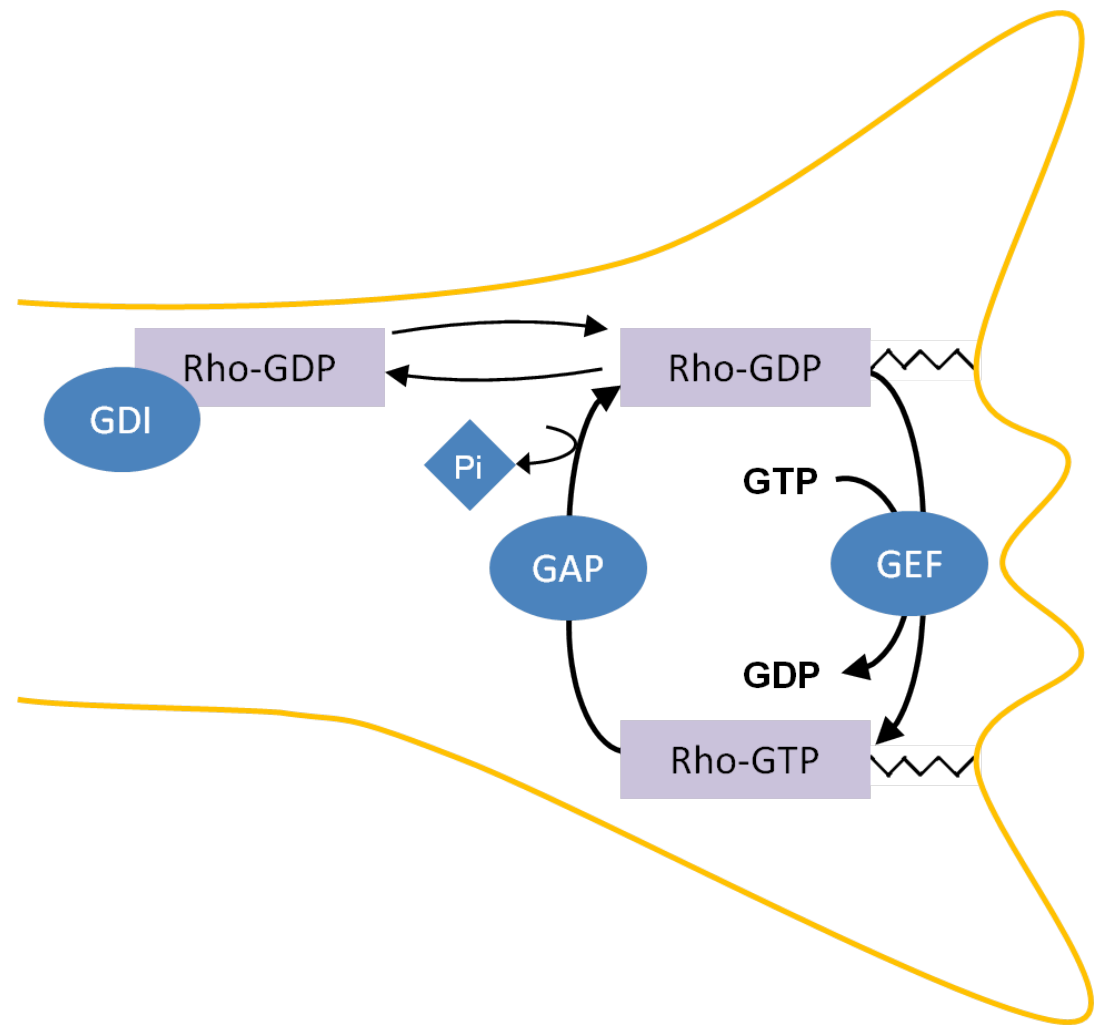

Schematic 1A. Regulation of Rho GTPases. The switch between the activity states of Rho GTPases is mediated by three classes of regulatory proteins, GEFs, GAPs and GDIs. Such tight regulation of Rho activity is crucial for the modulation of cytoskeletal dynamics in growth cone steering and axon extension.

The small Rho GTPases Rho, Rac and Cdc42 are modulators of cytoskeletal dynamics (Dickson, 2001; Govek et al., 2005; Luo, 2000) and primary effectors of neurotrophin signaling in axonal growth. In response to trophic stimuli, activated Trks bind to one of the GTPase regulatory proteins, guanine nucleotide exchange factors (GEFs), GTPase activating proteins (GAPs) or guanine nucleotide dissociation inhibitors (GDIs) to affect Rho GTPase activity (Miyamoto et al., 2006; Nakamura et al., 2002; Namekata et al., 2010). GEFs activate Rho GTPases by exchanging GDP for GTP, while GAPs facilitate inactivation by triggering GTP hydrolysis. GDIs regulate nucleotide exchange in concert with cytosol-membrane redistribution crucial for Rho GTPase activity (Luo, 2000) (Schematic 1A). While neurotrophin signaling activates Rac1 and $\mathrm{Cdc} 42$, the 'positive regulators' of axon and neurite growth, it triggers the inactivation of the 'negative regulator' RhoA. 
Additionally, neurotrophin-activated Trks may also stimulate direct phosphotidylinositol 3-kinase (PI3K)-binding to and activation of Rac1 and Cdc42 (Aoki et al., 2004; Nusser et al., 2002; Sarner et al., 2000). Activated Rac1 in turn, inhibits RhoA to promote neurite growth in neuronal cell lines and axon specification in primary neurons (Da Silva et al., 2003; Nusser et al., 2002). Once activated, the Rho GTPases recruit multiple downstream effectors to modulate cytoskeletal rearrangements for axonal morphogenesis. RhoA, Rac1 and Cdc42 effectors are illustrated in Schematic 1B.

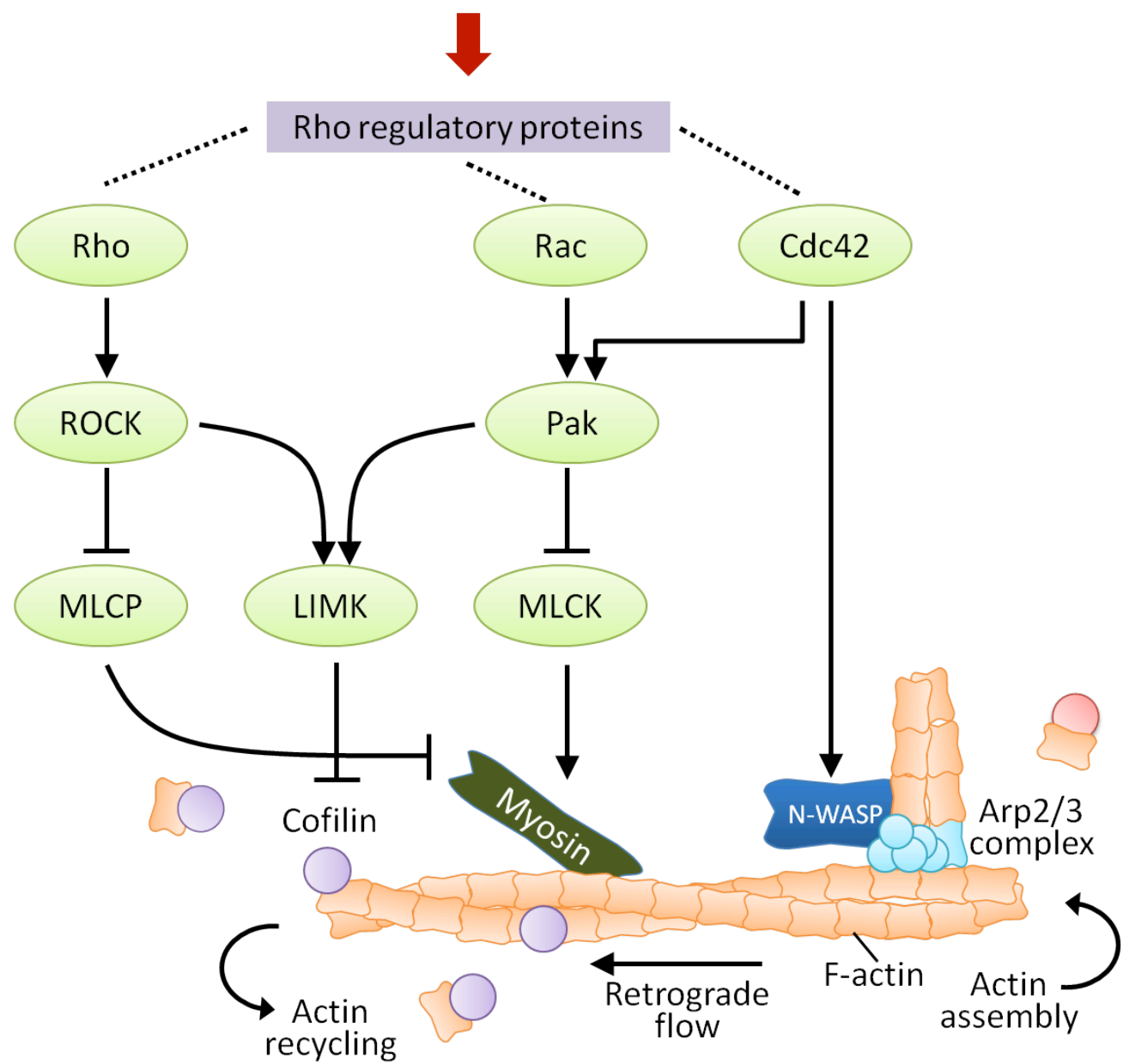

Schematic 1B. Effectors of Rho, Rac and Cdc42 in neurite and axon growth. Rho-GTP promotes neurite retraction by activating Rho kinase (ROCK). ROCK in turn, inhibits myosin light chain phosphatase (MLCP) to cause contraction of acto-myosin filaments, and activates Lim kinase (LIMK) to inhibit cofilin, shutting down actin recycling. Activated $\mathrm{Rac}$ and $\mathrm{Cdc} 42$ enhance neurite growth by binding to the effectors $\mathrm{p} 21$-activated kinase (PAK) and neuronal Wiscott Aldrich syndrome protein (N-WASP). While PAK inhibits myosin light chain kinase (MLCK) and myosin phosphorylation thereby reducing actomyosin contractility, N-WASP induces actin assembly. Additionally, PAK inhibits LIMK to locally inactivate cofilin and permit actin redistribution within the growth cone. Red arrow indicates extrinsic signaling. Modified from Dickson, 2001.

Aside from Rho GTPases, neurotrophins activate mitogen activated protein kinase (MAPK) and PI3K pathways in enhancing neurite and axon growth (Arevalo and $\mathrm{Wu}, 2006$; Atwal et al., 2000) (Schematic 1C). Phosphorylated MAPK induces cAMP-dependent progrowth transcriptional activity (Lonze and Ginty, 2002), while activated PI3K directly binds 
cytoskeletal proteins to regulate actin and microtubule dynamics for local axon assembly (Cosker and Eickholt, 2007; Goold and Gordon-Weeks, 2004).

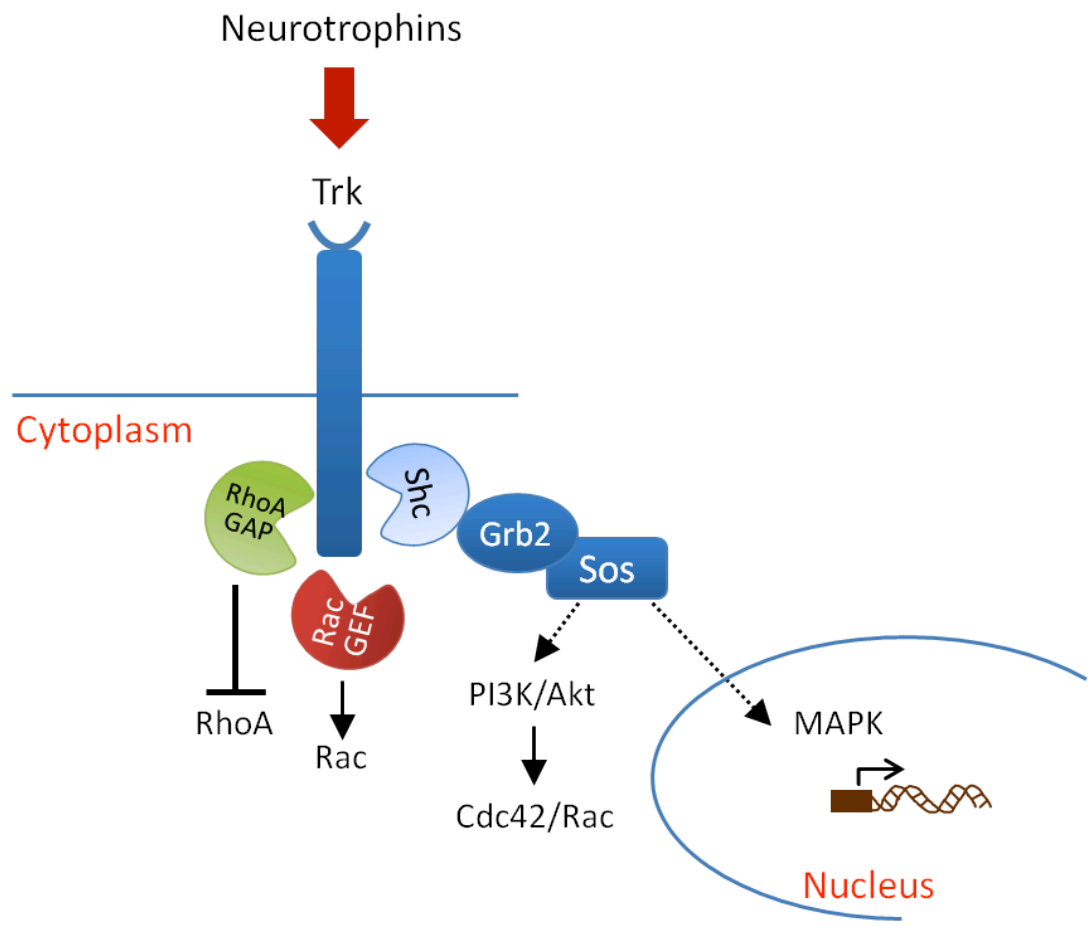

Schematic 1C. Neurotrophin signaling in neurite and axon growth. In response to neurotrophin signaling, the adaptors Shc/Grb2 together with a Ras GEF, Sos bind activated Trks and trigger intracellular cascades of the MAP and PI3 kinases. Besides, Rho regulatory proteins dock on phosphorylated tyrosine residues on Trks to modulate Rho GTPase activity.

\subsubsection{Fetters in axon regeneration: Glial inhibition of CNS regeneration}

The adult CNS and peripheral nervous system (PNS) respond differently to axon injury. While CNS axons have a very limited regenerative capacity, PNS neurons are regenerationcompetent. The basis for PNS regeneration after injury is rapid Wallerian degeneration ${ }^{*}$ (WD) supported by a quick breakdown and clearance of myelin debris by Schwann cells and macrophages at the injury site. In contrast, WD in CNS is delayed owing to the inability of oligodendrocytes to clear myelin, prolonging the exposure of the injured axon to an inhibitory environment (Vargas and Barres, 2007).

Such inhibitory milieu in CNS injury is composed of at least two extrinsic factors: (i) myelin, a lipid-rich component of oligodendrocytes that normally provides an insulating

\footnotetext{
* WD: The cellular and molecular events promoting axon degeneration and myelin clearance after injury
} 
ensheathment of neurons and (ii) glial scar, principally consisting of astrocytic aggregates, that forms at sites of lesion, both of which are enriched in growth-inhibitory molecules (Yiu and He, 2006).

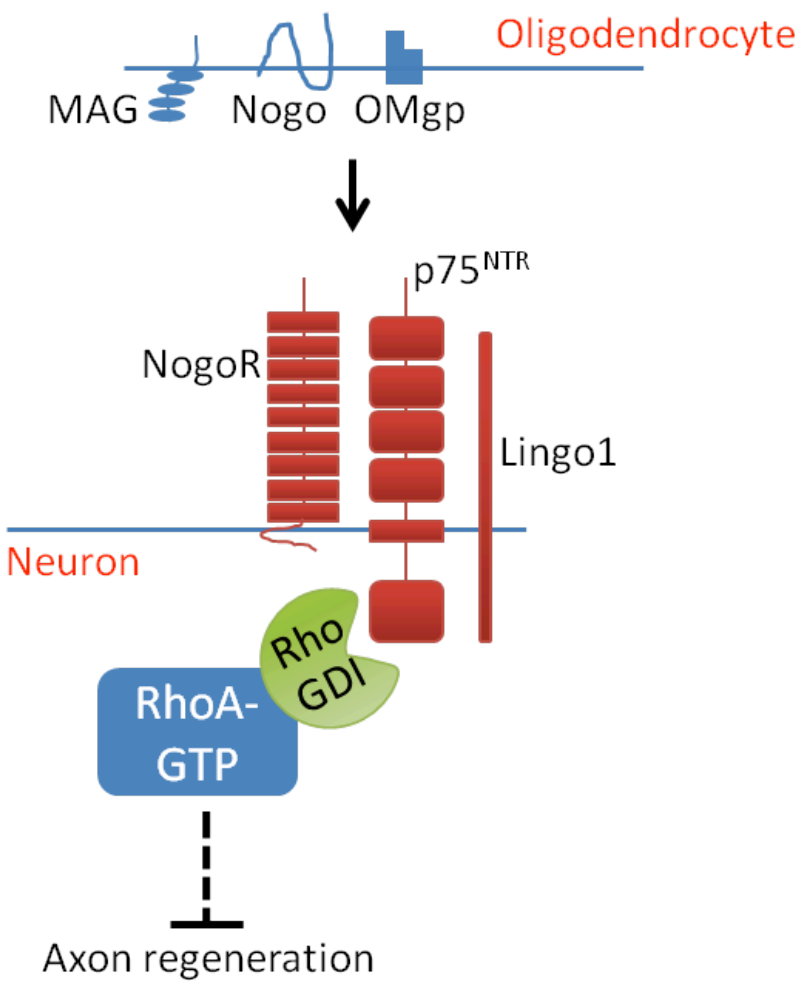

Schematic 1D. Myelin inhibition of axon regeneration. Myelin proteins Nogo, MAG and OMgp bind to the neuronal Nogo/p $75^{\mathrm{NTR}} /$ Lingo-1 tripartite receptor and induce growth cone collapse by activating Rho and its effector, ROCK.

Antibodies to myelin extracts led to the identification of the principal inhibitory component in myelin, later termed Nogo (Caroni and Schwab, 1988; Chen et al., 2000; GrandPre et al., 2000; Prinjha et al., 2000). Of the three isoforms, A, B and C, Nogo-A is the most CNS-abundant (Huber et al., 2002). Nogo-A associates with the brain-specific neuronal receptor, Nogo-R and ectopic expression of Nogo- $\mathrm{R}$ in neurons is sufficient to confer responsiveness to Nogo and mediate its inhibitory effects in axonal extension (Fournier et al., 2001). Follow-up studies identified other inhibitory proteins in myelin including myelin associated glycoprotein (MAG) and oligodendrocyte myelin glycoprotein (OMgp), all of which exert their effects by binding to Nogo-R (Yiu and He, 2006). In addition, Nogo receptor which lacks internal signal transducing activity is part of a receptor complex consisting of p75 neurotrophin receptor $\left(\mathrm{p} 75^{\mathrm{NTR}}\right)$ and Lingo-1 co-receptors that promote neuronal responses to myelin (Mi et al., 2004; Wang et al., 2002) (Schematic 1D). 
Glial scar is also rich in axon growth inhibitory factors, namely chondroitin sulphate proteoglycans (CSPGs), which are sulphated glycosaminoglycans released by reactive astrocytes at sites of injury. Importantly, both myelin- and CSPG-inhibition evoke neuronal responses by activating Rho GTPase and its effector, Rho kinase (ROCK). Selective inhibition of Rho using Clostridium botulinum $\mathrm{C} 3$ toxin promotes in vivo axon regeneration after optic nerve crush or spinal cord injury (Dergham et al., 2002; Lehmann et al., 1999), and enhances axon growth of retinal ganglion neurons on glial scar tissue in vitro (Monnier et al., 2003). Rho GTPase is thus, an unambiguous downstream component of extrinsic inhibition whose activation is enabled in part, by the Nogo co-receptor $75^{\mathrm{NTR}}$ (McGee and Strittmatter, 2003; Schwab, 2004; Yamashita and Tohyama, 2003; Yiu and He, 2006). Once activated, the Rho effector ROCK triggers a molecular cascade that eventually activates cofilin, an actindepolymerizing factor, to destabilize the actin cytoskeleton and induce growth cone collapse (Hsieh et al., 2006).

\subsection{Ubiquitination as a means of cell-intrinsic control of neuronal morphogenesis and axon regeneration}

Besides heavily relying on the environment, orchestration of neuronal morphogenesis also depends on cell-autonomous mechanisms for sustained effects on intracellular signaling. There is only a very recent revelation of intrinsic processes in axonal growth control, although extrinsic players viz. trophic factors have been extensively characterized.

Posttranslational modification (PTF) is a major program of intrinsic axon growth control besides epigenetic and transcriptional regulation of gene expression (Kawabe and Brose, 2011; Liu et al., 2012; Moore and Goldberg, 2011; Trakhtenberg and Goldberg, 2012). Following their synthesis, proteins may be chemically modified by the addition of functional groups such as phosphate, acetate, lipids or carbohydrates to change their fate, function or activity. Ubiquitination is one such PTF that refers to the covalent attachment of ubiquitin, a 76-amino acid protein, to substrates for various downstream events. There is a recent surge of research addressing ubiquitination as an intrinsic regulatory mechanism in neuronal development (Kawabe and Brose, 2011).

\subsubsection{The ubiquitination cascade and the $26 \mathrm{~S}$ proteasome}

The discovery of the mechanics of ubiquitination dates back to the 1980s when Avram Hershko, Aaron Ciechanover and Irwin Rose resolved the three-step ubiquitin-conjugation cascade. Ubiquitination soon came to be acknowledged as a 'ubiquitous' posttranslational 
modification that marks proteins for various cellular processes including proteasomal turnover, quality control, transcriptional activation, sorting, and functional modification (Hicke, 2001; Welchman et al., 2005). Ubiquitination is undisputedly, an emerging intrinsic regulatory mechanism crucial to axonal morphogenesis and regeneration.

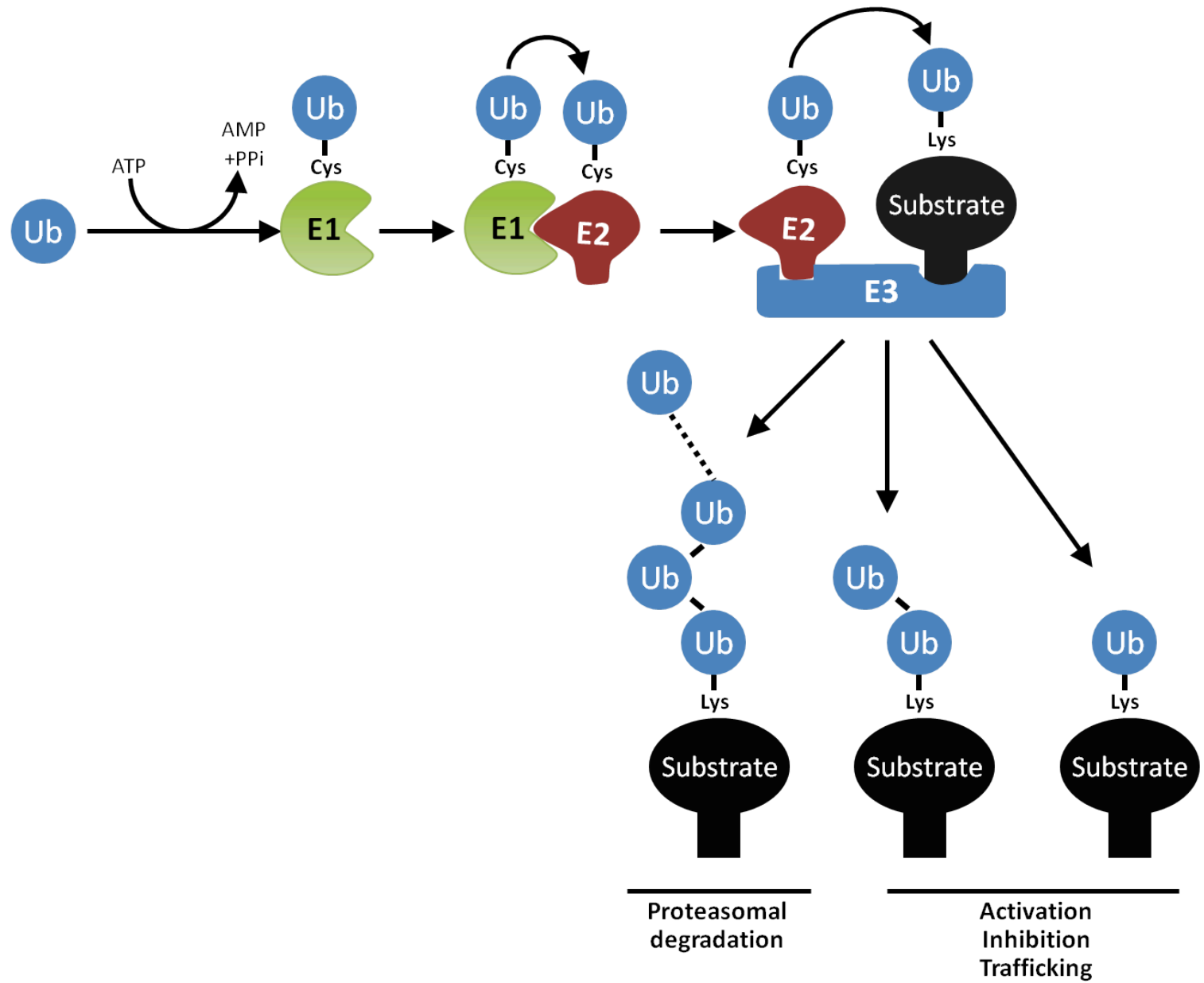

Schematic 1E. Ubiquitination cascade. Ubiquitination of proteins involves at least three enzymes that facilitate covalent attachment of ubiquitin to substrates. Protein modification thus, marks substrates for proteasomal degradation and other downstream events.

Ubiquitination initiates with ATP-dependent activation of ubiquitin (Ub). This step is catalyzed by the first enzyme of the cascade, E1 or the ubiquitin-activating enzyme. Subsequently, the activated ubiquitin is transferred to E2, the ubiquitin-conjugating enzyme following which, Gly76 of ATP-ubiquitin is conjugated to a lysine residue of a substrate protein by an amide-isopeptide bond. This last step is mediated by the E3 ubiquitin ligase (Hershko and Ciechanover, 1998). Using this mechanism, substrates may be mono-, di-, multior poly-ubiquitinated, the latter being the preferred modification for proteasomal turnover (Schematic 1E). Chain extension (poly-ubiquitination) may require an accessory factor, E4 (Koegl et al., 1999). 
The E3 ligases outnumber E1 and E2 enzymes several fold and the large repertoire ( 600) of E3 enzymes facilitate ubiquitination of an even larger number of substrates. The nature of the internal Ub-Ub lysine linkages in poly-ubiquitin chains provides additional information for further processing of substrates. A K48-linkage specific poly-ubiquitination, for instance, is indicative of proteasomal turnover (Chau et al., 1989).

Under most circumstances, poly-ubiquitination destines substrates for protein degradation by the $26 \mathrm{~S}$ proteasome. In eukaryotes, this organelle is a large multicatalytic protease that consists of a $20 \mathrm{~S}$ catalytic core $(\mathrm{CP})$ and a $19 \mathrm{~S}$ regulatory particle $(\mathrm{RP})$. The $\mathrm{CP}$ is a barrel-shaped structure made up of a stack of four heptameric rings (Groll et al., 1997). The proteolytically active subunits face the inner side of the 'barrel' to prevent non-specific degradation in the cell. The RP is one of the activator particles of the $\mathrm{CP}$ and possesses ubiquitin receptors for ubiquitinated-substrate recognition. By virtue of its deubiquitinase (DUB) and ATPase activity, the RP deubiquitinates and actively unfolds substrates and eventually slides them through a narrow channel that leads to the inner side of the CP (Groll et al., 2000; Smith et al., 2005; Verma et al., 2002; Yao and Cohen, 2002). The CP sequentially degrades substrates into short peptides in an ATP-dependent process (Kisselev et al., 1999). The peptides and ubiquitin are recycled.

\subsubsection{Ubiquitination in CNS development}

The role of ubiquitination in CNS function and disease is underscored by a multitude of studies that correlate pathological conditions with defective ubiquitination. Intense ubiquitin immunoreactivity in intracellular aggregates in Alzheimer's (AD) and Parkinson's (PD) disease brains points to a ubiquitin-proteasome system (UPS) dysfunction or an attempt of the UPS to clear the inclusions in neurodegeneration. Besides, aberrant E3 enzyme activity is a common feature of neurological disorders. To name a few, CHIP, Parkin and UBE3A are all E3 ligases whose mutations are associated with the pathology of PD, AD and the neurodevelopmental disorder, Angelman syndrome, respectively (Bingol and Sheng, 2011).

Posttranslational modification by ubiquitin is a recurrent theme in neurobiology that is gleaned from different aspects of neuronal development including neurogenesis, neuronal polarization, axon initiation and elongation, neuronal migration, dendritic development, axonal and dendritic pruning, synapse formation and synapse elimination (Kawabe and Brose, 2011). In the following text, I will summarize a few of the major findings in the context of axon and dendritic development. 
Components of the cytoskeleton are subject to regulation by ubiquitination in axon and dendritic growth. The RING ligase Rnf6 ubiquitinates the Rho/Rac effector LIM kinase 1 (LIMK1) at axon growth cones and inhibits axon outgrowth in hippocampal neurons in a proteasome-dependent manner (Tursun et al., 2005).

Smad ubiquitin regulatory factor-1 (Smurf1), a Homologous to E6-AP C-terminus (HECT) E3 ligase switches preference in degrading its two substrates, the polarity protein Par6 and RhoA in response to neurotrophins. Phosphorylation of Smurf1 enhances its preference of RhoA and local protein turnover of the Rho GTPase promotes axon extension (Cheng et al., 2011).

The HECT ligase neural precursor cell expressed developmentally down-regulated-4 (Nedd4) is known to mediate UPS-degradation of phosphatase and tensin homolog (PTEN), a negative regulator of PI3K signaling, to promote axonal branching in the frog (Drinjakovic et al., 2010). Rap2 GTPase is another substrate of Nedd4 contemporarily identified to regulate dendritic growth in the mouse (Kawabe et al., 2010). Here, Nedd4 catalyzes mono- or diubiquitination of Rap2 not to trigger its degradation but to inhibit the interaction of the small GTPase with its effector, Traf2 and Nck interacting kinase (TNIK). This stimulates dendritic growth and arborization (Kawabe et al., 2010).

A key intrinsic regulator of neuronal development is the multimeric RING E3 ligase anaphase promoting complex (APC). Originally identified as an essential regulator of cell cycle, the APC is now known to have various functions in postmitotic neurons, where it is abundantly expressed (Gieffers et al., 1999). The following section will address in detail, the role of APC in the CNS with an emphasis on Cdh1-APC, Cdh1 being one of the co-activators of the core.

\subsubsection{APC: a multisubunit RING ligase}

E3 ligases are determinants of substrate specificity and undoubtedly, crucial participants of ubiquitination. The E3 enzymes are classified into two main groups, based on their gross structure and catalytic activity, namely RING and HECT ligases. RING ligases contain an E2-binding RING domain and substrate-recognition elements, either on the same polypeptide (monomeric) or on different subunits (multimeric) (Weissman, 2001) (Schematic 1F). The HECT ligases contain in addition to the characteristic C-terminal HECT domain, substrate-binding motifs e.g. WW (PY-binding) and other domains such as C2 (phospholipidbinding) and RLD (chromatin and RanGTPase-binding). Functionally, HECT ligases are 
different from RING as they contain a catalytic Cys in their HECT domain that acts as an acceptor of ubiquitin from E2s. The ligase thus, engages in ubiquitin-intermediate formation before transferring ubiquitin to the substrate. In contrast, RING ligases passively mediate ubiquitin transfer by juxtaposing substrate and the ubiquitin-E2 complex (Rotin and Kumar, 2009).
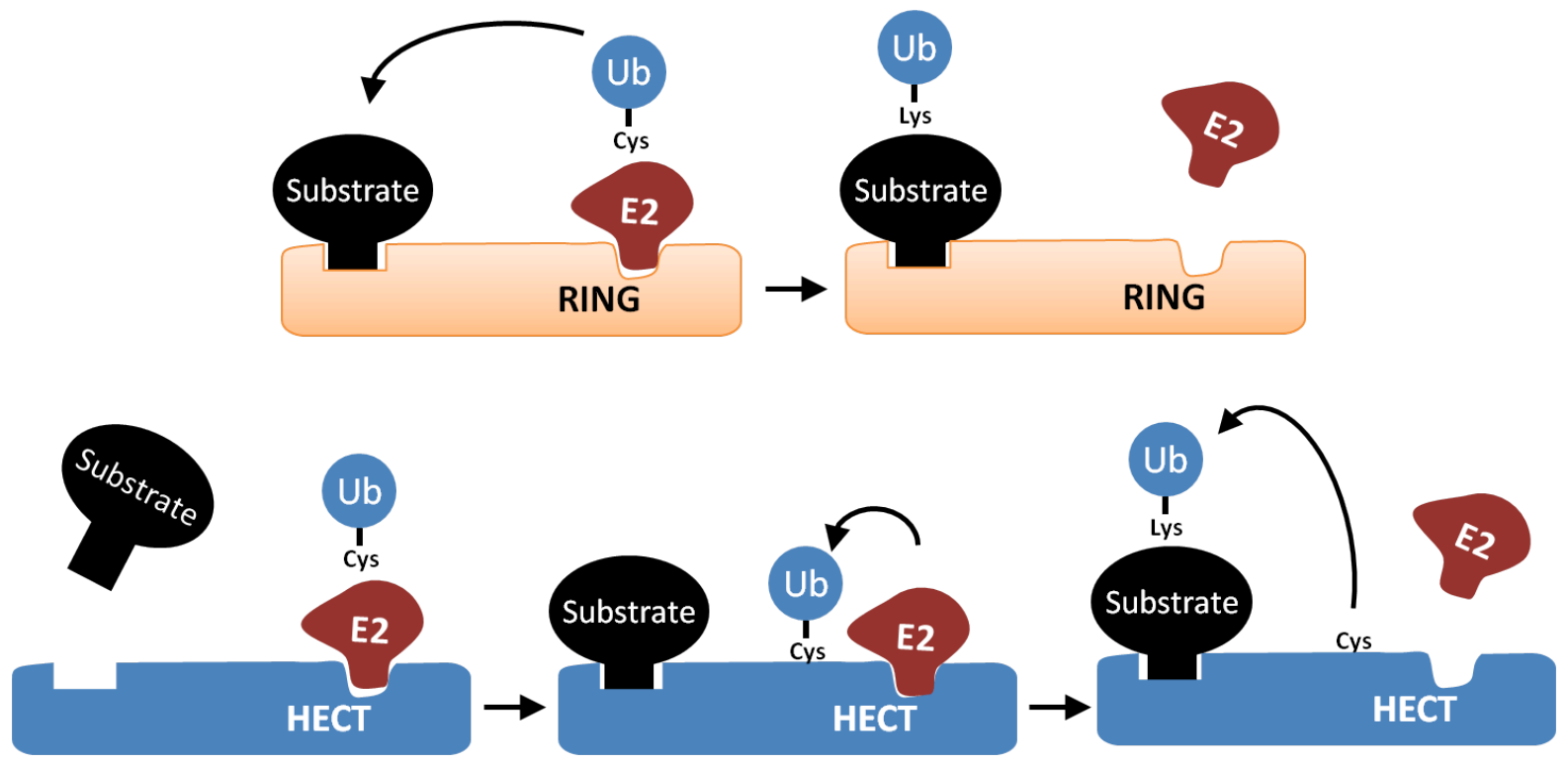

Schematic 1F. Mode of action of RING and HECT ligases. While RING ligases act as a scaffold to facilitate transfer of ubiquitin from E2 to substrate, HECT ligases harbor a catalytic Cys that acts as a ubiquitin acceptor and thus, actively participate in protein ubiquitination.

A third group of ligases called U-box E3s consist of fewer known enzymes with a RING domain-like U-box. Some theories suggest that U-box ligases behave as E4s. The prototypical U-box ligase C-terminus of Hsc70 interacting protein (CHIP) is involved in the clearance of misfolded proteins in certain pathological conditions (Cyr et al., 2002).

The anaphase promoting complex is a multisubunit RING E3 ligase that was first discovered to potentiate proteolytic degradation of cyclin B, in cell cycle regulation (Irniger et al., 1995; King et al., 1995). APC consists of at least 11 core subunits (Table 1A) but on its own has little activity requiring an association with one of its activators, Cdc20 (also called fizzy, fzy) or Cdc20 homolog 1 (Cdh1 also called fizzy-related, fzr) (Harper et al., 2002). 


\begin{tabular}{|c|c|c|}
\hline$\underline{\text { APC Subunit }}$ & Motifs & Function \\
\hline Apc1 & & $\begin{array}{l}\text { Bridges substrate-recognition and catalytic core } \\
\text { subcomplexes }\end{array}$ \\
\hline Apc2 & $\begin{array}{l}\text { Cullin } \\
\text { homology }\end{array}$ & $\begin{array}{l}\text { Scaffold, together with APC11 constitutes catalytic } \\
\text { core }\end{array}$ \\
\hline Apc3/Cdc27 & TPR repeats & Binds activators Cdh1, Cdc20 by their IR tails \\
\hline Apc4 & & Binds catalytic core and inhibits non-specific activity \\
\hline Apc5 & & Binds catalytic core and inhibits non-specific activity \\
\hline Apc6/Cdc16 & TPR repeats & \\
\hline Cdc26 & TPR repeats & Accessory subunit, stabilizes TPR subcomplex \\
\hline Apc $8 / \mathrm{Cdc} 23$ & TPR repeats & \\
\hline Apc10/Doc1 & $\begin{array}{l}\text { Doc domain, } \\
\text { IR tail }\end{array}$ & $\begin{array}{l}\text { Participates in substrate-recognition together with } \\
\text { activator }\end{array}$ \\
\hline Apc11 & RING finger & Binds E2s, constitutes catalytic core \\
\hline $\operatorname{Cdc} 20$ & $\begin{array}{l}\text { WD40 repeats, } \\
\text { IR tail }\end{array}$ & APC activator, substrate-binding, specificity factor \\
\hline Cdh1 & $\begin{array}{l}\text { WD40 repeats, } \\
\text { IR tail }\end{array}$ & APC activator, substrate-binding, specificity factor \\
\hline Apc7 & TPR repeats & $\begin{array}{l}\text { Accessory subunit, binds activators Cdh1, Cdc } 20 \text { by } \\
\text { their IR tails }\end{array}$ \\
\hline
\end{tabular}

Table 1A. APC subunits, their motifs and functions.

\subsubsection{Composition of APC}

APC2 and APC11 constitute the "catalytic core" and the heterodimer is sufficient to bind to E2s and stimulate non-specific ubiquitination (Gmachl et al., 2000; Leverson et al., 2000; Tang et al., 2001). APC1, 4 and 5 which bind APC2 and APC11 to make an APC subcomplex likely inhibit the non-specific activity of the core. This necessitates activator- binding for APC activation. Cdc27/APC3, Cdc16/APC6, APC7 and Cdc23/APC8 are tetratricopeptide repeat (TPR) polypeptides that exist as homo-dimers in the "substrate-recognition competent" sub-complex. Here, Cdc27 and APC7 directly associate with the conserved C-terminal 'IR tail' of the activators Cdh1 or Cdc20 (Vodermaier et al., 2003). Another member of the catalytic 
core, Doc1/APC10 also possesses an IR tail. While both Cdh1 and Cdc20 enable substrate interaction by means of C-terminal WD repeats (Kraft et al., 2005), APC10 also participates in

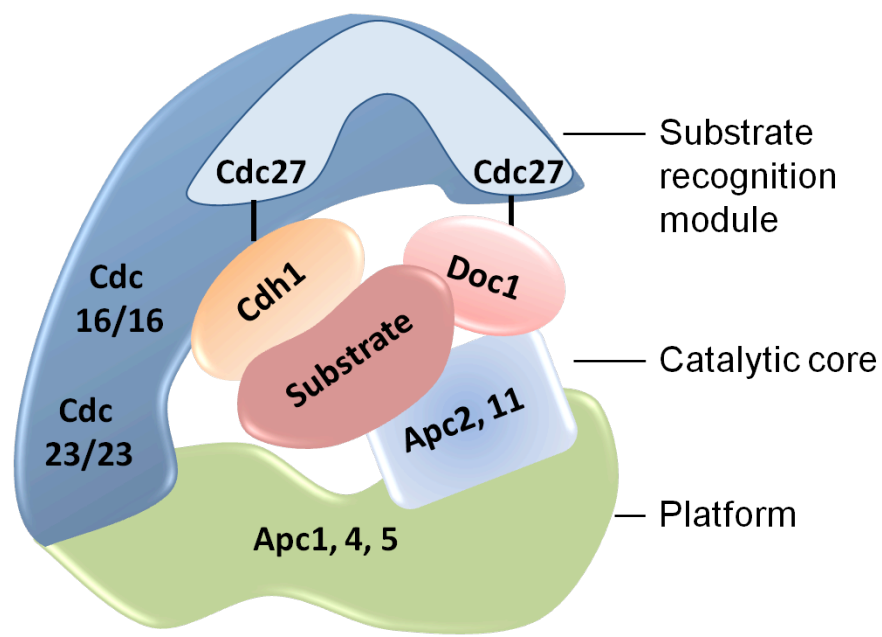

Schematic 1G. Structure of APC. The substrate-recognition subcomplex comprising TPR proteins and activator connect to the catalytic core Apc2 and Apc11 by virtue of a 'platform' of Apc1, Apc4 and Apc5 subunits. The TPR protein Cdc27 binds both activator, and Doc1 of the catalytic core. The latter proteins both contribute to substrate recognition. Modified from Foe and Toczyski, 2011.

substrate recognition together with the activators (da Fonseca et al., 2011; Thornton et al., 2006; Vodermaier et al., 2003). Using high-resolution cryo-electron microscopy, a pseudoatomic model of the APC was recently proposed (da Fonseca et al., 2011; Schreiber et al., 2011) and the exact placement of the different subunits was resolved (Schematic 1G).

\subsubsection{Canonical role of APC}

APC was first discovered for its role in degrading mitotic cyclins. Cdc20 and Cdh1 activate APC at two distinct phases of the cell cycle. Cdc20-APC activity is detected at the onset of anaphase where it is implicated in the destruction of securin, to relieve the inhibition on proteolysis essential for sister chromatid separation (Hauf et al., 2001; Uhlmann et al., 1999), and Clb5, an S-phase cyclin (Shirayama et al., 1999). Cdh1 is activated and binds APC towards late mitosis (Jaspersen et al., 1999; Visintin et al., 1998) when cyclin-dependent kinase (Cdk) activity is low. By promoting the proteolysis of Cdc20 itself (Fang et al., 1998; Prinz et al., 1998) and of the mitotic cyclin Clb2 (Schwab et al., 1997), Cdh1-APC signals mitotic exit. Cdh1-APC activity continues into the G1 phase where Cdh1 mediates the destruction of the bulk of mitotic cyclins and prevents premature cell progression into $\mathrm{S}$ phase. Thus, the switch from $\mathrm{Cdc} 20$ to $\mathrm{Cdh} 1$ allows APC to regulate cyclin/Cdk levels and ensure a successful anaphase and subsequent mitotic exit. 


\subsubsection{Substrate recognition by APC}

The two activators of the APC, Cdh1 and Cdc20 confer most of the substrate specificity to the RING ligase. Their interaction with substrates is facilitated by the presence of 'signature motifs' or degrons on target sequences as described below.

The first indication of a conserved motif in substrates that signals APC binding and proteolysis came from B-cyclins, which harbor a 9-amino acid (AA) sequence $\underline{\mathrm{RXX}} \underline{\mathrm{LXXXX}} \underline{\mathrm{N}}$ at their $\mathrm{N}$-terminus with at least 3 highly conserved residues. This was termed the "destruction box" (D-box), fusion of which can trigger proteolysis of an unrelated protein (Glotzer et al., 1991). Eventually, it came to be known that A and B-type cyclins differ slightly in the respective D-boxes in that the last Asn is only weakly conserved in cyclin A (King et al., 1996). This led to the generalization that the D-box has a consensus $\underline{R} X X \underline{L}$ motif with or without a third conserved Asn, five residues downstream.

Some years later, a second degenerate motif, the "KEN" box was identified (Pfleger and Kirschner, 2000). Analysis of deletion mutants of human Cdc20, a Cdh1-APC target that lacks a D-box, led to the identification of a 100-AA N-terminal peptide that exhibits a wildtype profile in ubiquitination assays. Subsequently, the degron was pinned down to a KEN sequence at position 97, mutation of which completely blocks ubiquitination as well as recognition by Cdh1-APC. As with the D-box, the KEN motif is also transposable to unrelated proteins (Pfleger and Kirschner, 2000). D-box and KEN box are signature elements on both Cdh1 and Cdc20 substrates (Glotzer et al., 1991; Hendrickson et al., 2001; Jacobs et al., 2001; Sczaniecka et al., 2008; Zur and Brandeis, 2001).

Additional rare unique motifs have been identified on Cdh1 substrates. Aurora-A (AurA), a mitotic kinase and a Cdh1 substrate, contains a third kind of recognition motif the A-box. The sequence comprises a conserved serine (Ser53) that is phosphorylated during M-phase. A hyperphosphorylation mutant (S53D) evades Cdh1-dependent destruction of Aur-A and potentially interferes with mitotic exit (Littlepage and Ruderman, 2002; Littlepage et al., 2002). A CRY degron with the consensus CRYXPS is another Cdh1-recognition motif identified in mammalian Cdc20 (Reis et al., 2006).

Some substrates require multiple degrons for proper APC recognition and degradation (Burton and Solomon, 2001; Hendrickson et al., 2001; Zur and Brandeis, 2001). The D and KEN motifs may also function as bipartite recognition signals for Cdh1 and Cdc20 (Burton and Solomon, 2001), as is observed for the budding yeast cell cycle kinase Hsl1. Hsl1 contains a 
D-box, essential for its interaction with Cdc20 and a KEN box that associates with Cdh1. Disrupting any of the motifs can stabilize the protein. Also, not all proteins with a Cdh1 or Cdc20 recognition motif are potential APC substrates. This is supported by the identification of 'pseudosubstrate inhibitors' of Cdh1 viz. Emil and Acm1, both of which harbor D- and/or KEN boxes that bind Cdh1 and interfere with bona fide substrate-recruitment (Miller et al., 2006; Ostapenko et al., 2008).

\subsubsection{Regulators of Cdh1-APC}

Phosphorylation is the principal mode of regulation of Cdh1-APC. In budding yeast, mitotic Cdk1 (Cdc28) together with G1/S-phase cyclins phosphorylate and inactivate Cdh1 in the S and M phases (Jaspersen et al., 1999; Zachariae et al., 1998). Phosphorylation of Cdh1 triggers nuclear export thereby dissociating Cdh1 from the APC core (Jaquenoud et al., 2002). In late mitosis, when Cdk1 levels drop, dephosphorylation by protein phosphatase Cdc14 activates Cdh1 (Jaspersen et al., 1999).

In cortical neurons, Cdh1 is inhibited by phosphorylation at Ser-40, Thr-121 and Ser163 by Cdk5 upon a glutamate/N-methyl D-aspartate (NMDA)-excitotoxic stimulus (Almeida et al., 2005; Maestre et al., 2008). Such an inhibitory phosphorylation depletes the nuclear pool of Cdh1 and leads to an accumulation of the substrate cyclin B1. The latter mediates NMDA receptor (NMDAR)-dependent neuronal excitotoxicity and apoptotic death (Almeida et al., 2005).

Besides phosphorylation, Cdh1 levels are subject to auto-regulation by proteolysis. Cdh1-APC catalyzes its own ubiquitination during G1 and G0 phases of the cell cycle for selfdestruction. Two D-boxes at its N-terminus appear to mediate its degradation, mutations of which block Cdh1-ubiquitination and significantly stabilize the protein (Listovsky et al., 2004).

Other regulators include the early mitotic inhibitor-1 (Emi1), which acts as a 'pseudosubstrate' to inhibit Cdh1-APC. Emil accumulates in late G1 phase and promotes S-phase entry by directly binding Cdh1 and inactivating it (Hsu et al., 2002; Reimann et al., 2001).

\subsubsection{Cdh1-APC: Emerging roles in neuronal development and regeneration}

The observation by Jan-Michael Peters and colleagues that Cdh1-APC is active in postmitotic neurons of the brain (Gieffers et al., 1999) set the stage for a new era of research in the APC field, which was until then confined to cell cycle regulation. 
In their pioneering study, Konishi et al. uncovered a crucial role for Cdh1-APC in axon growth and cerebellar patterning in the rodent brain (Konishi et al., 2004). Inhibiting Cdh1 levels either by a Cdh1 RNAi or by expression of the APC inhibitor Emil causes a significant increase in axonal length in primary neurons as compared to control suggesting that $\mathrm{Cdh} 1$ is an inhibitor of axonal growth in postmitotic neurons. Additionally, Cdh1 also controls patterning of granule neurons in vivo, given that Cdh1 knockdown causes parallel fiber defasciculation in the molecular layer of the cerebellum (Konishi et al., 2004).

Subsequently SnoN, a Ski/Sno family transcriptional co-regulator, was identified as a substrate of Cdh1-APC in axon growth control (Stegmuller et al., 2006). Unlike Cdh1, SnoN promotes axon growth in postmitotic neurons. Ubiquitination of SnoN by Cdh1 in a D-box dependent manner stimulates SnoN degradation and contributes to Cdh1 inhibition of process growth. Besides, loss of SnoN in vivo results in defective parallel fiber development. Further, the Cdh1-APC/SnoN pathway intercalates with TGF- $\beta$ signaling (Stegmuller et al., 2008). Knockdown of Smad2, an effector of TGF- $\beta$ pathway produces a similar long axon phenotype as Cdh1 RNAi and this effect is antagonized by SnoN RNAi. While Smad2 and Cdh1 act in concert upstream of SnoN, Ccd1, an actin-binding protein, regulates axon growth downstream of SnoN (Ikeuchi et al., 2009), along a multi-levelled pathway of axon growth regulation.

In the same year that SnoN emerged as a Cdh1-substrate, inhibitor of DNA-binding-2 (Id2), an inhibitor of bHLH transcription factors, was discovered as a second Cdh1-target in axon growth regulation (Lasorella et al., 2006). Id2, highly unstable in neurons, contains a canonical D-box and is a direct substrate of Cdh1. Id2 D-box mutant (Id2 DBM) is markedly stabilized and is a strong stimulator of axonal growth (Lasorella et al., 2006). It is not known whether SnoN and Id2 act in independent pathways downstream of Cdh1-APC in the control of axonal morphogenesis. The identification of multiple substrates of Cdh1-APC in axon growth bolsters the concept of multimodal regulation of cellular events by E3 ligases.

The fact that Cdh1-APC acts as an intrinsic brake in controlling axon growth, persuaded researchers to foresee a putative role for $\mathrm{Cdh} 1$ in axon regeneration. In line with this hypothesis, Cdh1 knockdown stimulates axon growth on myelin (Konishi et al., 2004), a major inhibitor of axon regeneration after injury (McKerracher et al., 1994; Mukhopadhyay et al., 1994; Schwab, 2004). Also, the Cdh1-target Id2 inhibits expression of anti-axonal growth genes including NogoR and prevents activation of RhoA (Lasorella et al., 2006), a crucial downstream component of myelin signaling (Dergham et al., 2002; Lehmann et al., 1999). 
In 2011, a study demonstrated Id2 DBM-induced axon regeneration after spinal cord injury (SCI), shedding light on a role for $\mathrm{Cdh} 1$ in regenerative therapy (Yu et al., 2011). Id2 DBM overexpressing dorsal root ganglion (DRG) neurons grow into the sites of lesion after T10 spinal cord hemisection. Moreover, Id2 DBM expression greatly reduces axonal dieback in ascending sensory fibers after SCI.

\begin{tabular}{|c|c|c|}
\hline$\underline{\text { Substrate }}$ & Function & References \\
\hline SnoN & Inhibition of axonal growth & $\begin{array}{l}\text { (Stegmuller et al., } \\
\text { 2006) }\end{array}$ \\
\hline Id2 & $\begin{array}{l}\text { Inhibition of axonal growth, } \\
\text { axon regeneration }\end{array}$ & $\begin{array}{l}\text { (Lasorella et al., 2006; } \\
\text { Yu et al., 2011) }\end{array}$ \\
\hline Liprin- $\alpha$ & Control of synaptic size and synaptic activity & $\begin{array}{l}\text { (van Roessel et al., } \\
\text { 2004) }\end{array}$ \\
\hline GluR1 & $\begin{array}{l}\text { Control of GLR-1 glutamate receptor abundance in } \\
\text { C.elegans, EphA4-dependent down-regulation of } \\
\text { GluR1 density and synaptic strength in rodents }\end{array}$ & $\begin{array}{l}\text { (Fu et al., 2011; Juo } \\
\text { and Kaplan, 2004) }\end{array}$ \\
\hline Unknown & $\begin{array}{l}\text { Late-long term potentiation (L-LTP), } \\
\text { memory and cognitive function }\end{array}$ & $\begin{array}{l}\text { (Kuczera et al., 2011; } \\
\text { Li et al., 2008) }\end{array}$ \\
\hline Cyclin B & Pro-survival & (Almeida et al., 2005) \\
\hline $\begin{array}{l}\text { Fasciclin } 2 \\
\text { (putative) }\end{array}$ & Glial migration in Drosophila & $\begin{array}{l}\text { (Silies and Klambt, } \\
\text { 2010) }\end{array}$ \\
\hline Loco & Glia differentiation in Drosophila & (Kaplow et al., 2008) \\
\hline Pfkfb3 & $\begin{array}{l}\text { Down-regulation of glycolysis; } \\
\text { protective against oxidative stress and apoptosis }\end{array}$ & $\begin{array}{l}\text { (Herrero-Mendez et } \\
\text { al., 2009; Rodriguez- } \\
\text { Rodriguez et al., } \\
\text { 2012) }\end{array}$ \\
\hline
\end{tabular}

Table 1B. Functions of Cdh1-APC in neuronal and glial development.

Aside from its role in axon growth and regeneration, Cdh1-APC is also recognized for other functions in neuronal and glial development and disease. Its involvement in regulating synapse size and homeostatic plasticity by targeting synaptic proteins is well known (Fu et al., 2011; Juo and Kaplan, 2004; van Roessel et al., 2004). Cdh1-APC participates in ephrindependent maintenance of homeostatic plasticity during chronic elevated activity in excitatory neurons. In response to ephrin signaling, Cdh1 ubiquitinates the AMPA receptor GluR1 for 
proteolytic degradation. The decreased density of surface GluR1 thereof, results in a reduced synaptic strength and helps restore homeostasis (Fu et al., 2011).

An emerging role for Cdh1-APC is in learning and memory. Cdh1 heterozygotes show defective late phase-long term potentiation (L-LTP) suggestive of cognitive decline (Li et al., 2008). Knockout mice that lack an essential APC-subunit APC2, exhibit impaired spatial memory, implicating a role for either Cdc20- or Cdh1-APC or both in hippocampus-dependent learning and memory (Kuczera et al., 2011). In addition, the APC2 knockouts are incapable of fear memory extinction, a feature of neuropsychiatric illnesses such as phobia and posttraumatic stress disorder (PTSD). This observation suggests a potential role of the ligase in the pathology of anxiety disorders (Kuczera et al., 2011).

Cdh1-APC is reported as a pro-survival factor in postmitotic neurons and its reduced expression is correlated with apoptosis after global cerebral ischemia (Almeida et al., 2005; Zhang et al., 2011). In protecting neurons from cell death, Cdh1 may serve to keep a check on cyclin B1 levels and prevent terminally differentiated neurons from a forced S-phase re-entry (Almeida et al., 2005).

Functions of Cdh1-APC in neuronal and glial development are listed in Table 1B.

\subsection{Overcoming extrinsic inhibition by manipulating intrinsic growth capacity}

The adult CNS has a very limited regenerative potential. Any effort of an injured neuron to sprout and regrow even in a permissive environment is extremely limited (Harel and Strittmatter, 2006; Liu et al., 2011; Yiu and He, 2006) suggesting an intrinsic inability of adult CNS neurons to sustain growth processes. This intrinsic loss of growth capacity occurs perinatally when neurons terminally differentiate (Chen et al., 1995; Goldberg et al., 2002).

Whether manipulating intrinsic growth state can rescue neurons from extrinsic inhibition is a long-standing question in regenerative therapy. However, the 'conditioning lesion' paradigm of spinal cord DRG neurons offers clues in support of the same. DRG neurons possess two axonal branches: a central branch incapable of regeneration that connects to the spinal cord, and a regeneration-competent peripheral branch that innervates peripheral targets. A conditioning lesion viz. a previous injury to the peripheral axon, predisposes the axotomized central branch to regenerative responses and stimulates growth on a peripheral nerve graft despite the nonpermissive environment (Neumann and Woolf, 1999). 
In a conditioning lesion, transcriptional programs are altered such that growthpromoting genes are activated and neuronal activity genes are down-regulated (Hoffman, 2010). Additionally, intracellular cAMP levels may be transiently elevated to facilitate cAMPresponse gene transcription and this appears to be crucial for axon growth on myelin and MAG (Neumann et al., 2002; Qiu et al., 2002). Taken together, directing the intrinsic state of the neuron enables axon regeneration even in the hostile environment of the CNS. In recent research, adult axon regeneration in vivo has been achieved by smothering intrinsic inhibition.

Null mice for zinc-finger transcription factor Kruppel like factor-4 (KLF-4), an intrinsic axon growth inhibitor, show robust RGC axon regeneration after optic nerve crush (Moore et al., 2009). Likewise, PTEN deletion in vivo enhances RGC axon growth across lesion after optic nerve injury (Park et al., 2008). PTEN is an inhibitor of mammalian target of rapamycin (mTOR), a potent stimulator of cap-dependent protein translation essential for cell growth (Guertin and Sabatini, 2005; Hoeffer and Klann, 2010). By activating mTOR pathway, PTEN loss potentiates re-growth after axotomy. A regenerative role for Cdh1-APC has been proposed recently (Yu et al., 2011). Id2 DBM overexpression is propitious for DRG axon recovery after spinal cord injury presumably because it can overcome intrinsic inhibition by Cdh1-APC (Lasorella et al., 2006; Yu et al., 2011).

\subsection{Cdh1-APC: A signaling node for extrinsic and intrinsic cascades}

It is clear that both extracellular and cell-autonomous processes affect axonal development and regeneration. Cdh1-APC that classically keeps a check on cell-cycle entry is abundantly expressed in postmitotic neurons and presents a cell-intrinsic leash on postmitotic growth (Gieffers et al., 1999; Konishi et al., 2004), characteristic of differentiation factors. In doing so, the E3 ligase arrests growth-promoting transcriptional programs involving SnoN and Id2 (Lasorella et al., 2006; Stegmuller et al., 2006). In the first instance, Cdh1-APC collaborates with TGF- $\beta$ signaling and together with regulatory Smads inhibits SnoN (Stegmuller et al., 2008). However, how Cdh1-APC interfaces with extrinsic growthpromoting trophic factors and growth-inhibitory molecules in regulating neuronal development is yet elusive.

As with axonal development, axon re-growth after injury is also subject to tight regulation. The adverse environment in the CNS represents a major curb in axon regeneration (Yiu and He, 2006). This is exacerbated by an intrinsic growth inability in mature neurons 
owing to an accumulation of inhibitory factors such as Cdh1-APC. Indeed relieving such an inhibition potentiates axon growth on nonpermissive substrates (Konishi et al., 2004).

Thus, the first aim of my project is to study how Cdh1-APC in regulating axon growth, intercalates with trophic stimuli by careful analyses of potential cross-talks between the E3 ligase and the effectors of trophic signaling namely MAPK, PI3K and small Rho GTPases. The second aim of my project is to identify downstream players in Cdh1-APC cascade that may circumvent myelin inhibition and address a direct effect of Cdh1-APC on the activity of RhoA, the key cellular component of extrinsic inhibition (McGee and Strittmatter, 2003; Schwab, 2004; Yiu and He, 2006). 


\section{Materials and Methods}

\subsection{Reagents and lab equipment}

Chemicals used in this study were purchased from Applichem (Darmstadt), Merck (Darmstadt), Roth (Karlsruhe) or Sigma Aldrich (Steinheim) unless otherwise indicated. Cell culture media and supplements were ordered from GIBCO $^{\circledR}$, Life Technologies ${ }^{\mathrm{TM}}$ (Darmstadt). Enzymes were purchased from either New England Biolabs (Frankfurt am Main) or Fermentas (St. Leon Rot). DNA and protein ladders were also from Fermentas.

Micropipettes were bought from Gilson (Limburg-Offheim) and other plasticware were from Eppendorf (Hamburg), Sarstedt AG (Nürnbrecht), Becton Dickinson (Heidelberg) or Greiner Bio-One (Frickenhausen). Nucleospin ${ }^{\circledR}$ Xtra Midi/Maxi EF kits for plasmid DNA preparations were purchased from Macherey-Nagel (Düren).

Hardware such as power packs and thermocycler were ordered from Biometra, (Göttingen), heating blocks and centrifuges from Eppendorf, rocker and shaker from Heidolph (Schwabach) and electrophoretic apparatus from Bio-RAD (Munich).

\subsection{Plasmids and primers}

Plasmids and primers used in this study are listed in Appendices 1 and 2.

\subsection{Buffers and stock solutions}

HHGN: 1x HBSS (Gibco), 2.5 mM HEPES pH 7.5, 35 mM glucose, 4 mM NaHCO 3 Annealing buffer: $100 \mathrm{mM} \mathrm{CH}{ }_{3} \mathrm{COOK}, 30 \mathrm{mM}$ HEPES-KOH, $2 \mathrm{mM}\left(\mathrm{CH}_{3} \mathrm{COO}\right)_{2} \mathrm{Mg}$

2x HBSS: 50 mM HEPES pH 7.05-7.11, $10 \mathrm{mM} \mathrm{KCl,} 280 \mathrm{mM} \mathrm{NaCl}, 15 \mathrm{mM}$ glucose, $1.5 \mathrm{mM}$ $\mathrm{Na}_{2} \mathrm{HPO}_{4}$

BME+insulin: $35 \mathrm{mM}$ glucose, $1 \mathrm{X}$ PSG, $10 \mu \mathrm{g} / \mathrm{ml}$ insulin in BME

Triton ${ }^{\circledR}$ X-100 lysis buffer: $150 \mathrm{mM} \mathrm{NaCl}, 50 \mathrm{mM}$ Tris-HCl, $1 \mathrm{mM}$ EDTA, $1 \%$ Triton ${ }^{\circledR} \mathrm{X}$ 100

Co-IP buffer: $150 \mathrm{mM} \mathrm{NaCl}, 20 \mathrm{mM}$ Tris-HCl pH 7.4, 1mM EDTA, 1\% Nonidet P-40, 10\% glycerol 
RIPA buffer: $150 \mathrm{mM} \mathrm{NaCl}, 50 \mathrm{mM}$ Tris- $\mathrm{HCl} \mathrm{pH} 7.5,1 \%$ sodium deoxycholate, $1 \%$ Nonidet P-40, 0.1\% SDS

SDS-sample buffer: $300 \mathrm{mM}$ Tris- $\mathrm{HCl} \mathrm{pH}$ 6.8, 10\% SDS, 50\% glycerol, 25\% $\beta$-mercapto ethanol, $0.05 \%$ bromophenol blue

Buffer A: $10 \mathrm{mM}$ HEPES, $10 \mathrm{mM} \mathrm{KCl}, 0.1 \mathrm{mM}$ EDTA, $0.1 \mathrm{mM}$ EGTA, $0.5 \mathrm{mM}$ phenylmethylsulphonyl fluoride (PMSF)*, $1 \mathrm{mM}$ dithiothrietol (DTT)*, $5 \mu \mathrm{g} / \mathrm{ml}$ aprotinin*

Buffer A+: Buffer A, 0.1\% Nonidet P-40

Buffer C: 20 mM HEPES pH 7.9, 400 mM NaCl, 1 mM EDTA, 1 mM EGTA, 1 mM PMSF*, $1 \mathrm{mM} \mathrm{DTT}^{*}, 5 \mu \mathrm{g} / \mathrm{ml}$ aprotinin*

Lower buffer: $1.5 \mathrm{M}$ Tris $\mathrm{pH} 8.8,0.4 \% \mathrm{SDS}$

Upper buffer: $0.5 \mathrm{M}$ Tris $\mathrm{pH} 6.8,0.4 \%$ SDS

Running buffer: $125 \mathrm{mM}$ Tris, $1.25 \mathrm{M}$ glycine, $0.5 \%$ SDS

Transfer buffer: $48 \mathrm{mM}$ Tris, $39 \mathrm{mM}$ glycine, $0.0375 \%$ SDS, 20\% methanol

10X PBS pH 7.4: $1.37 \mathrm{M} \mathrm{NaCl}, 14.7 \mathrm{mM} \mathrm{KCl}, 78.1 \mathrm{mM} \mathrm{Na}_{2} \mathrm{HPO}_{4}, 26.8 \mathrm{mM} \mathrm{KH}_{2} \mathrm{PO}_{4}$

PBST: 1x PBS, $0.1 \%$ Tween-20

Mounting medium: 50\% glycerol, 5\% N-propyl gallate in PBS

Genotyping buffer: $200 \mathrm{mM} \mathrm{NaCl}, 10 \mathrm{mM}$ Tris $\mathrm{pH}$ 8.0, $10 \mathrm{mM}$ EDTA, 0.5\% SDS

2x TAE pH 8.5: $80 \mathrm{mM}$ Tris-acetate, 2 mM EDTA

2x YT (1000 ml): $16 \mathrm{~g}$ tryptone, $10 \mathrm{~g}$ yeast extract, $5 \mathrm{~g} \mathrm{NaCl}$

* added fresh before use

\subsection{Cell culture}

\subsubsection{Cell lines}

All cell culture work was performed under sterile conditions in a safety cabinet (HERAsafe $^{\circledR}$, Thermo Scientific). HEK 293T cells were cultured in DMEM (GIBCO ${ }^{\circledR}$, Life Technologies $^{\mathrm{TM}}$, Catalog no. 11960) supplemented with 10\% FCS and $2 \mathrm{mM}$ penicillin, 
streptomycin and glutamine (PSG). Cultures were maintained at $37^{\circ} \mathrm{C}$ and $5 \% \mathrm{CO}_{2}$ in a $\mathrm{CO}_{2^{-}}$ incubator (HERAsafe ${ }^{\circledR}$, Thermo Scientific, Bonn). Cells were passaged in $10 \mathrm{~cm}$ dishes or 6well plates when 90-95\% confluent. The cells were washed in PBS and incubated with $1 \mathrm{ml}$ of trypsin-EDTA at $37^{\circ} \mathrm{C}$ for $5 \mathrm{~min}$. The enzymatic reaction was arrested by adding cold DMEM containing serum. The cell suspension was centrifuged at $4^{\circ} \mathrm{C}$ at $800 \mathrm{rpm}$ for $5 \mathrm{~min}$. The supernatant was discarded and the cell pellet was resuspended in $5 \mathrm{ml}$ of fresh medium. The cell suspension was plated at 1:5 dilution on a $10 \mathrm{~cm}$ plate ('rescue') or 6-well plates appropriate for transfection.

\subsubsection{Primary neuronal culture}

Cerebellar granule neurons were isolated from postnatal Wistar rats (postnatal day 6 or P6) as described previously (Bilimoria and Bonni, 2008), and maintained in BME (GIBCO ${ }^{\circledR}$, Life Technologies ${ }^{\mathrm{TM}}$, Catalog no. 41010) supplemented with 10\% calf serum (Hyclone Laboratories, Logan, UT), $25 \mathrm{mM} \mathrm{KCl}$ and $2 \mathrm{mM}$ PSG. Neurons were plated on polyornithinecoated plates or glass coverslips, 30 million cells per 6-well plate and 24 million cells per 24well plate. For morphological analyses, 20 million cells were plated per 24 -well plate and kept in 'conditioned medium' or with glucose, PSG and $10 \mu \mathrm{g} / \mathrm{ml}$ insulin referred to as 'BME+insulin'. At P6+1 day in vitro (DIV 1), neurons were treated with $10 \mu \mathrm{M}$ of the mitotic inhibitor cytosine $\beta$-D-arabinofuranoside to prevent proliferation of non-neuronal cells and at DIV 3, with $25 \mathrm{mM}$ glucose to replenish the carbon source.

For myelin coating, polyornithine-coated glass coverslips were incubated with 40 $\mu \mathrm{g} / \mathrm{ml}$ of myelin (isolated from the brains of 3-6 months old mice) in PBS overnight at $4^{\circ} \mathrm{C}$. Purified myelin was kindly provided by Mostafa Bakhti, Simons Lab, Max Planck Institute of Experimental Medicine (MPI-EM), Göttingen.

Cortical neuron culture was performed as described previously (Bhakar et al., 2002).

\subsection{Molecular cloning}

\subsubsection{Generation of NES- and NLS-Smurf1 rescue constructs}

\subsubsection{Annealing of primer pairs}

Primers for the generation of nuclear exclusion signal (NES) and nuclear localization signal (NLS)-Smurf1 rescue constructs are listed in Appendix 2. The HA-NLS-Smurf1 and HA-NES-Smurf1 rescue plasmids were constructed by introducing two tandem repeats of SV40 large T-antigen NLS and HIV-1 Rev nuclear export signal (Yoneda et al., 1999), 
respectively, between EcoRI and KpnI sites of pCMV5-HA-Smurf1-Res plasmid. Additionally, the internal NES in Smurfl (Tajima et al., 2003) was mutated (I612A, L614A) in the HA-NLS-Smurf1 rescue plasmid as described in section 2.6.

Sense and antisense primer pairs were diluted to a final concentration of $2 \mu \mathrm{M}$ each in a $50 \mu \mathrm{l}$ volume of annealing buffer. Annealing of primer pairs was performed using the following program on a PCR block:

\begin{tabular}{lll}
\hline \multicolumn{1}{c}{ Step } & \multicolumn{1}{c}{ Temperature } & Time [min:sec] \\
\hline \hline Denaturation & $95^{\circ} \mathrm{C}$ & $04: 00$ \\
\hline Annealing & $72^{\circ} \mathrm{C}$ & $10: 00$ \\
\hline Slow cool to $25^{\circ} \mathrm{C}$ & $0.1^{\circ} \mathrm{C} / \mathrm{sec}$ & -- \\
\hline
\end{tabular}

\subsubsection{Phosphorylation of annealed oligos}

$20 \mu \mathrm{l}$ of the annealed oligos was mixed with 10x kinase buffer, $10 \mathrm{U}$ polynucleotide kinase and ATP at a final concentration of $1 \mathrm{mM}$ in a total volume of $50 \mu \mathrm{l}$ and the reaction was incubated at $37^{\circ} \mathrm{C}$ for $30 \mathrm{~min} .5 \mu \mathrm{l}$ of this reaction was used for ligation.

\subsubsection{Preparation of the vector and ligation reaction}

$3 \mu \mathrm{g}$ of pCMV5-HA-Smurf1-Res plasmid was digested with KpnI and EcoRI and purified using Nucleospin ${ }^{\circledR}$ Extract II Kit (Macherey-Nagel). The digested vector was treated with calf intestinal phosphatase for $1 \mathrm{~h}$ at $37^{\circ} \mathrm{C}$ for dephosphorylation of 5 ' phosphate groups. Following another column-purification, the digested vector was eluted in $40 \mu 1$ deionized water. $0.5 \mu \mathrm{l}$ of the eluate was then used in a $20 \mu \mathrm{l}$ volume ligation reaction together with $5 \mu 1$ phosphorylated insert, 5 U T4 DNA ligase and 10x ligase buffer. Ligation was performed overnight at $16^{\circ} \mathrm{C}$.

\subsubsection{Bacterial transformation and selection of positive clones}

Chemically-competent E.coli DH5 $\alpha$ cells were transformed with the ligation reaction. Positive clones were identified by digesting mini-preparations of plasmid DNA (Nucleospin ${ }^{\circledR}$ Plasmid QuickPure, Macherey-Nagel) with SacI and AgeI. A shift in the 700 bp band indicates presence of the insert. 


\subsubsection{Generation of Myc-ubiquitin-p250GAP and Myc-p250GAP-ubiquitin fusion constructs}

A 768 bp KpnI-PshAI fragment (fragment A) at the N-terminus of p250GAP was PCR amplified from pCDNA3-6xMyc-p250GAP-3'UTR. This was ligated in pGEM $^{\circledR} T$ Easy vector (Promega, Mannheim) according to the manufacturer's instructions. The PstI site in the vector was mutated by site-directed mutagenesis as described below. Next, human ubiquitin was PCR amplified from a pCMV5-HA-ubiquitin plasmid. The PCR product was cloned into the PstI site of fragment A, the PstI site is found 7 nucleotides upstream of the p250GAP ATG. The ubiquitin-fragment A in pGEMT Easy was sub-cloned in the original vector pCDNA3-6xMycp250GAP-3'UTR using KpnI and PshAI to obtain the Myc-ubiquitin-p250GAP construct.

To generate Myc-p250GAP-ubiquitin fusion construct, a 1916 bp C-terminal fragment (fragment B) was PCR amplified from pCDNA3-6xMyc-p250GAP-3'UTR using BsrG1 and AflII primers. This was introduced into pGEMT-Easy vector. Using site-directed mutagenesis, the p250GAP stop codon within fragment B was mutated and replaced with a HpaI site. Human ubiquitin was amplified from the pCMV5-HA-ubiquitin plasmid using HpaI primers. A stop codon was included in the reverse primer between the ubiquitin-annealing region and HpaI site. The PCR product was cloned in the HpaI site in fragment B. Fragment B now containing ubiquitin at the C-terminus of p250GAP was re-inserted into the original vector using BsrG1 and AflII. All primers, plasmids and their source are listed in Appendices 1 and 2.

Vector digestion and dephosphorylation, ligation and bacterial transformation at all steps were performed as described in sections 2.5.1.3 and 2.5.1.4.

\subsection{Site-directed mutagenesis (SDM)}

Primers for SDM were designed as per the guidelines in the QuickChange ${ }^{\circledR}$ SiteDirected Mutagenesis Kit (Stratagene, Waldbronn). A list of primers used in the generation of Smurf1 rescue construct, p250GAP and Smurf1 D-box mutants, Smurf1 NES mutant, RhoA K6,7R and p250GAP-ubiquitin fusion constructs can be found in Appendix 2. The sample reaction was prepared as indicated below: 


\begin{tabular}{ll}
\hline Component & Volume $(\mu \mathrm{l})$ \\
\hline \hline 10x Pfu buffer (Fermentas) & 5.00 \\
\hline DNA template (20ng) & $\mathrm{x}$ \\
\hline $10 \mu \mathrm{M}$ sense primer & 1.25 \\
\hline $10 \mu \mathrm{M}$ antisense primer & 1.25 \\
\hline $25 \mathrm{mM}$ dNTP mix & 0.40 \\
\hline $2.5 \mathrm{U}$ Pfu DNA polymerase (Fermentas) & 1.00 \\
\hline Final volume (deionized water) & 50.0 \\
\hline
\end{tabular}

SDM was performed using the following program on a PCR block:

\begin{tabular}{llll}
\hline Segment & Cycles & Temperature & Time [min:sec] \\
\hline \hline $\mathbf{1}$ & 1 & $95^{\circ} \mathrm{C}$ & $00: 30$ \\
\hline $\mathbf{2}$ & 18 & $95^{\circ} \mathrm{C}$ & $00: 30$ \\
\cline { 3 - 4 } & & $55^{\circ} \mathrm{C}$ & $01: 00$ \\
\cline { 3 - 4 } & & $72^{\circ} \mathrm{C}$ & $02: 00 / \mathrm{kb}$ plasmid \\
\hline $\mathbf{3}$ & 1 & $72^{\circ} \mathrm{C}$ & $05: 00$ \\
\hline
\end{tabular}

The reaction was quick-chilled on ice for $2 \mathrm{~min}$. The PCR product was digested at $37^{\circ} \mathrm{C}$ for 30 min with $1 \mu \mathrm{l}$ of DpnI to cleave the methylated non-mutated parental DNA template. 1 $\mu \mathrm{l}$ of the digested sample was used to transform competent DH5 $\alpha$ cells. Positive clones were identified by sequencing mini-preparations of plasmid DNA.

\subsection{Plasmid transfections}

\subsubsection{HEK 293T cells}

HEK 293T cells were transfected using the modified calcium phosphate method (Sambrook and Russell, 2006). Cells were 60-70\% confluent at the time of transfection. To prepare the calcium phosphate-DNA co-precipitate for one well of a 6-well plate, 0.2-1.0 $\mu \mathrm{g}$ of plasmid DNA together with a GFP expression plasmid in $90 \mu \mathrm{l}$ deionized water was mixed with $10 \mu \mathrm{l}$ of $2.5 \mathrm{M} \mathrm{CaCl}_{2}$. The calcium phosphate-DNA solution was mixed with an equal volume of $2 x$ HBSS at RT. The solution was mixed well by swirling with a pipette tip and pipetting up and down and then incubated at RT for $5 \mathrm{~min}$. The DNA precipitate was then added to the medium above the cell monolayer. The plate was gently rocked to mix the 
medium and incubated at $37^{\circ} \mathrm{C}$ in a humidified incubator for 2-4 days until biochemical analyses. Transfection efficiency was monitored by checking for GFP fluorescence using a Nikon Eclipse TS100 epifluorescence microscope (Nikon, Tokyo).

\subsubsection{Primary neurons}

Primary granule neurons were transfected at DIV 0 ( $8 \mathrm{~h}$ after plating) for morphological assays or at DIV 3 for biochemical analyses using the modified calcium phosphate method detailed. Conditioned medium on the neurons was collected in a $50 \mathrm{ml}$ tube prior to transfection and stored at $37^{\circ} \mathrm{C}$. The neurons were washed twice with pre-warmed DMEM. Fresh DMEM was added and the plate was placed in a humidified $37^{\circ} \mathrm{C}$ incubator and the neurons were serum-starved for 45 min. During this time, plasmid DNA precipitate was prepared as described above. For morphological assays, neurons on $12 \mathrm{~mm}$ coverslips were transfected with a total of $2 \mu \mathrm{g}$ plasmid together with $0.3 \mu \mathrm{g}$ plasmid encoding $\mathrm{BCL}_{\mathrm{XL}}$, an antiapoptotic protein, and less than $10 \%$ of a GFP expression plasmid. The expression of $\mathrm{BCL}_{\mathrm{XL}}$ has little or no effect on axon length (Konishi et al., 2004). After 45 min of serum starvation, the precipitate was added to the medium and the neurons were incubated at $37^{\circ} \mathrm{C}$ for another 12-18 min. The serum-free medium was aspirated and the neurons were washed twice with DMEM and replaced with conditioned medium or BME+insulin (where indicated). The transfected neurons were continued to be incubated at $37^{\circ} \mathrm{C}$ until fixation at DIV 3/DIV 4 for morphological assays or until cell lysis at DIV 6.

The transfection ensures that about $85 \%$ of the GFP-positive neurons co-express two other plasmids.

\subsection{Biochemical experiments}

\subsubsection{Protein lysates and Bradford assay}

To prepare whole cell lysates of HEK 293T or primary neurons, the cells were washed once with PBS and lysed in Triton ${ }^{\circledR}$ X-100 lysis buffer with freshly added protease inhibitors ( $1 \mu \mathrm{g} / \mathrm{ml}$ of leupeptin, aprotinin, pepstatin and $1 \mathrm{mM} \mathrm{DTT})$. Lysates were incubated on ice for $30 \mathrm{~min}$ and then centrifuged at $13,000 \mathrm{rpm}$ at $4^{\circ} \mathrm{C}$ for $5 \mathrm{~min}$. The supernatant (total protein) was transferred to fresh $1.5 \mathrm{ml}$ microcentrifuge tubes and subjected to Bradford assay.

The Bradford reagent (Bio-RAD) was diluted 1:5 in PBS. 3, 6, 9 and $12 \mu \mathrm{g} / \mathrm{ml}$ of BSA served as standards. The lysate was diluted 1:500 or 1:1000 in the Bradford solution. OD $_{595}$ was measured using a spectrophotometer (GE Healthcare, Munich) and a calibration curve was 
plotted using the $\mathrm{OD}_{595}$ values for the standards. The concentration of the diluted sample protein was estimated from its $\mathrm{OD}_{595}$ value based on the calibration curve and then multiplied by the dilution factor to obtain the concentration of the undiluted protein. Typically, 30-50 $\mu \mathrm{g}$ of total protein was used for expression analysis and $800 \mu \mathrm{g}-1 \mathrm{mg}$ of protein for coimmunoprecipitation or cell-based ubiquitination assays.

\subsubsection{Co-immunoprecipitation (Co-IP) analysis}

For exogenous Co-IP assays, HEK 293 T cells were cultured on 6-well plates (2 wells per condition). After 2-3 days of transfection, cells were lysed in $150 \mu \mathrm{l} /$ well of Co-IP buffer with freshly added protease inhibitors. For endogenous Co-IP, total brain lysates (in Co-IP buffer) were obtained from P9 wild-type mice. Total protein was estimated as described above. Equal amounts of protein $(800 \mu \mathrm{g}-1 \mathrm{mg})$ from different conditions were incubated with $0.8-$ $1.0 \mu \mathrm{g}$ of first antibody for $4 \mathrm{~h}$ at $4{ }^{\circ} \mathrm{C}$ in a tumbler. $5 \%$ of the lysate was boiled in SDS-sample buffer at $95^{\circ} \mathrm{C}$ and served as the input. Protein A Sepharose ${ }^{\mathrm{TM}}$ (GE Healthcare) beads were washed twice with Co-IP buffer and then resuspended in an equal volume of Co-IP buffer. 20 $\mu l$ of equilibrated beads was added to the lysate after $4 \mathrm{~h}$ incubation with the first antibody. The sample was left in a tumbler at $4^{\circ} \mathrm{C}$ for another $1 \mathrm{~h}$ following which it was briefly spun down $(10,000 \mathrm{rpm}, 30 \mathrm{sec})$ and the supernatant was discarded. The protein-bound beads were washed with $500 \mu \mathrm{l}$ of Triton ${ }^{\circledR} \mathrm{X}-100$ buffer, spun again and washed another two times to remove any unbound protein. For more stringent washes, RIPA buffer was used. The beads were subjected to a final wash with PBS. The sample was spun down, supernatant removed and the bound protein was eluted by boiling in $30 \mu \mathrm{l}$ of SDS-sample buffer at $95^{\circ} \mathrm{C}$ for $5 \mathrm{~min}$. The sample was analyzed by SDS-PAGE and Western blotting.

\subsubsection{Subcellular fractionation}

Granule neurons were cultured on $6 \mathrm{~cm}$ plates and 2-3 plates were used for subcellular fractionation. Neurons were washed once with PBS and lysed in a total of $400 \mu$ of cold Buffer A with freshly added protease inhibitors (0.5 mM PMSF, 5-10 $\mu \mathrm{g} / \mathrm{ml}$ aprotinin and 5 mM N-ethyl maleimide (NEM)). The lysate was collected in a $1.5 \mathrm{ml}$ microcentrifuge tube and placed on ice for 15 min following which it was finely homogenized using a dounce tissue grinder. The lysate was then centrifuged at $2000 \mathrm{rpm}$ for $5 \mathrm{~min}$ at $4^{\circ} \mathrm{C}$. The supernatant (postnuclear supernatant fraction) was transferred to a fresh tube. The pellet was resuspended in Buffer $\mathrm{A}+$ and centrifuged as before. The pellet was dissolved in $50 \mu \mathrm{l}$ of Buffer $\mathrm{C}$ and incubated on a tumbler for $15 \mathrm{~min}$ at $4^{\circ} \mathrm{C}$. The sample was then spun at $14,000 \mathrm{rpm}$ for $20 \mathrm{~min}$ 
and the supernatant (nuclear fraction) was transferred to a fresh tube. Both subcellular fractions were analyzed by SDS-PAGE and Western blotting. Besides the protein of interest, the fractions were also probed for appropriate controls for nuclear ( $\mathrm{SnoN}$ ) and post-nuclear supernatant (14-3-3 $\beta)$ fractions.

\subsubsection{Cell-based ubiquitination assays}

Cell-based ubiquitination assays were performed using lysates of HEK 293T cells cultured on 6-well plates ( 2 wells per condition). The cells were lysed 2 days after transfection in $150 \mu \mathrm{l} /$ well of RIPA buffer with fresh protease inhibitors and $10 \mathrm{mM}$ of the deubiquitinase inhibitor NEM. Total protein was estimated using Bradford assay. After saving 5\% lysate for input, equal amounts of protein from different conditions were incubated with the first antibody in a tumbler for $2 \mathrm{~h}$ at $4^{\circ} \mathrm{C} .20 \mu \mathrm{l}$ of Protein A Sepharose ${ }^{\mathrm{TM}}$ beads equilibrated with RIPA buffer was added to the tubes and the samples were continued to be rotated for another $45 \mathrm{~min}$. The samples were briefly spun down (10,000 rpm, $30 \mathrm{sec})$ and washed as described above (Section 2.8.2). The bound protein was eluted by boiling in SDS-sample buffer. The samples were analyzed using SDS-PAGE and Western blotting. The membrane was probed using a mouse monoclonal anti-ubiquitin antibody (Santa Cruz, Heidelberg).

\subsubsection{Pharmacological treatment of granule neurons}

For axon assays involving inhibition of PI3K activity, granule neurons were treated with $20 \mu \mathrm{M}$ LY294002 (Cell Signaling, Frankfurt am Main) at DIV 1 for $48 \mathrm{~h}$, following which they were fixed and subjected to immunocytochemistry. For MEK inhibition, neurons were treated with $5 \mu \mathrm{M}$ U0126 (Calbiochem ${ }^{\circledR}$, Merck Millipore) at DIV 1 for $48 \mathrm{~h}$ until fixation.

For proteasome inhibition, neurons were treated with $5 \mu \mathrm{M}$ lactacystin (Calbiochem) or vehicle or $10 \mathrm{nM}$ bortezomib (LC Laboratories, Woburn, MA) for $10 \mathrm{~h}$ at DIV 2. For exogenous proteins, neurons were transfected at DIV 3 with HA-Smurf1 WT or the Smurf1 Dbox mutants (DBM1-5) together with a GFP expression plasmid and treated with lactacystin (or vehicle) at DIV 6. Lysates were prepared in Triton ${ }^{\circledR}$ X-100 buffer and equal amounts of total protein were analyzed by SDS-PAGE and Western blotting.

\subsubsection{SDS-PAGE and Western Blotting}

Glass plates and gel-casting apparatus were purchased from Bio-RAD. Denaturing poly-acrylamide gels were prepared as outlined below: 


\begin{tabular}{lccccc}
\hline \multicolumn{1}{c}{$\mathbf{2 x}$} & \multicolumn{3}{c}{ Separating Gel (ml) } & Stacking gel (ml) \\
\hline \multicolumn{1}{c}{$\%$ v/v } & $\mathbf{7 . 5}$ & $\mathbf{8}$ & $\mathbf{1 0}$ & $\mathbf{1 2}$ & $\mathbf{3 . 9}$ \\
\hline \hline $\mathbf{3 0 \%}$ Acrylamide & 3.75 & 4.00 & 5.00 & 6.00 & 0.65 \\
\hline Lower buffer & & $-----3.75------$ & 1.25 \\
\hline Water & 7.5 & 7.25 & 6.25 & 5.25 & 3.05 \\
\hline APS & $-----50-60 \mu 1-----$ & $30 \mu 1$ \\
\hline TEMED & $------5-6 \mu 1-----$ & $2-3 \mu 1$ \\
\hline
\end{tabular}

$7.5,8,10$ or $12 \%$ separating gels were used depending on the molecular size of the protein/s of interest. The samples were subjected to electrophoresis at a constant current of 40 $\mathrm{mA}$ per gel until the sample front reached the end of the separating gel. Protein transfer was performed using the wet-blot system onto a nitrocellulose membrane for $90 \mathrm{~min}$ at a constant amperage of $250 \mathrm{~mA}$. The transfer apparatus was disassembled and the membrane was blocked with $4 \% \mathrm{w} / \mathrm{v}$ non-fat milk in PBST for $15 \mathrm{~min}$ at RT. After a quick wash with PBST, the membrane was incubated with appropriate primary antibodies, diluted in PBST $(+3 \%$ BSA, $+0.2 \% \mathrm{w} / \mathrm{v} \mathrm{NaN}_{3}$ ), on a rocker for $1 \mathrm{~h}$ at $\mathrm{RT}$ or overnight at $4{ }^{\circ} \mathrm{C}$. Primary antibodies used for immunoblotting are listed in Appendix 3. Following incubation with primary antibody, the membrane was washed three times with PBST, each time for $10 \mathrm{~min}$ on a rocker and subsequently incubated with HRP-conjugated anti-mouse or anti-rabbit secondary antibody (Dianova, Hamburg), diluted 1:5000 in 4\% milk in PBST. Following 30 min incubation with the secondary antibody at RT, the membrane was washed another 3 times with PBST. About $400 \mu 1$ of each of ECL ${ }^{\mathrm{TM}}$ Western blotting substrate and enhancer solutions (Thermo Scientific, Bonn) were mixed and applied onto the membrane. The membrane was dried and sealed in fresh wrap and placed in a photographic film cassette. An X-ray film exposed to the chemiluminescent reaction for $5 \mathrm{~min}, 3 \mathrm{~min}$ and 30 seconds, was fixed and developed in a dark room to visualize the protein bands of interest. Exposure time was varied depending on the intensity of the bands.

\subsubsection{Luciferase assays}

HEK 293T cells cultured on 24-well plates (3 wells per condition) were transfected with Renilla-Smurf1 WT or Renilla-Smurf1 DBM3/4 expression plasmid together with SV40 firefly luciferase (pGL3 basic promoter) expression plasmid that served as the internal control. After 2 days of transfection, cells were lysed in $100 \mu 1 /$ well of passive lysis buffer (Promega). 
The lysates were rocked at RT for $20-30 \mathrm{~min}$, spun down at $14,000 \mathrm{rpm}$ at $4{ }^{\circ} \mathrm{C}$ for another 10 min and the supernatant was transferred to fresh tubes. $30 \mu \mathrm{l}$ of the sample was applied in triplicates to a 96-well plate. A dual luciferase assay was performed in collaboration with Dr. Moritz Rossner, MPI-EM, Göttingen.

\subsection{Immunocytochemistry}

The standard protocol for GFP staining of transfected neurons is described below. Neurons on cover slips were washed twice with PBS and fixed with 4\% PFA in PBS for 10 min. All steps were performed at RT. Neurons were permeabilized with $0.4 \% \mathrm{v} / \mathrm{v}$ Triton ${ }^{\circledR} \mathrm{X}$ 100 in PBS for $10 \mathrm{~min}$. After two PBS washes, neurons were incubated with blocking buffer (BME+10\% HS) for $30 \mathrm{~min}$. Cover slips were carefully transferred to a $20 \mathrm{~cm}$ petri dish covered with aluminium foil onto a wet Whatmann paper. Rabbit polyclonal anti-GFP antibody (Life Technologies ${ }^{\mathrm{TM}}$ ) in blocking buffer was applied to the cells and incubated for 1 h. The cover slips were washed and incubated with Cy2 or Alexa 488-conjugated anti-rabbit antibody (Dianova) in blocking buffer. After 30 min incubation, the cover slips were washed again with PBS. The cells were counterstained with dye bisbenzimide Hoechst 33258 for 5 min. The cover slips were briefly washed with distilled water and mounted onto mounting medium on glass slides. Immunostained neurons were observed under a Nikon Eclipse Ti epifluorescence microscope and subjected to axon growth assays.

For Smurf1 immunostaining, neurons were subjected to immunocytochemistry using a mouse monoclonal Smurfl antibody (Sigma Aldrich) as described previously (Cheng et al., 2011). Active RhoA, total RhoA and HA immunostaining were performed according to the manufacturer's instructions using mouse monoclonal antibodies to RhoA-GTP (NewEast Biosciences, King of Prussia, PA), RhoA (Abcam) and HA (Covance), respectively. For endogenous p250GAP staining, granule neurons were fixed and permeabilized in ice-cold methanol for $10 \mathrm{~min}$ at $-20^{\circ} \mathrm{C}$. Neurons were blocked in $10 \%$ NGS in PBS. Rabbit antip250GAP antiserum (version 2f-1, Dr. Takanobu Nakazawa, Tokyo) diluted to $2 \mu \mathrm{g} / \mathrm{ml}$ in $1.5 \%$ NGS in PBS was applied to the cells and incubated overnight at $4{ }^{\circ} \mathrm{C}$. The neurons were washed and incubated with secondary antibody in $1.5 \%$ NGS and proceeded with counterstaining as described above.

\subsection{Morphological assays}

Axon growth assays were performed as described previously (Konishi et al., 2004; Stegmuller et al., 2006). Images of GFP-positive neurons were captured by a blinded observer 
using the green channel with a 20x objective. Axon growth was analyzed by measuring the axonal (longest process) lengths using ImageJ software. The lengths were obtained in pixels and converted to $\mu \mathrm{m}(316$ pixels $=100 \mu \mathrm{m})$. Statistical analyses (t-test or ANOVA) were performed using the Graph Pad Prism 5.0c software. The mean values were plotted and error bars represent mean + SEM.

\subsection{Confocal imaging}

RhoA/GFP staining in hippocampal neurons was visualized using a Leica TCS SP2 confocal microscope with a $63 x$ oil objective. To evaluate RhoA and GFP intensity, the mean grey values at regions of interest were estimated using ImageJ software.

\subsection{Cdh1 mouse line}

The Cdh1 gene trap mouse line was kindly provided by Pumin Zhang (Baylor College of Medicine, Texas, USA). Owing to embryonic lethality of Cdh1 knockouts, all experiments were carried out in the Cdh1-heterozygotes $\left(\mathrm{Cdh}^{+/-}\right)$and the wildtype $\left(\mathrm{Cdh} 1^{+/+}\right)$littermates.

\subsubsection{Isolation of genomic DNA and genotyping}

\begin{tabular}{ll}
\hline Component & Volume $(\boldsymbol{\mu l})$ \\
\hline \hline $5 \mathrm{x}$ GoTaq ${ }^{\circledR}$ buffer (Promega) & 4.00 \\
\hline DNA template (10ng) & $\mathrm{x}$ \\
\hline $10 \mu \mathrm{M}$ sense primer (wildtype/mutant) & 1.00 \\
\hline $10 \mu \mathrm{M}$ antisense primer (wildtype/mutant) & 1.00 \\
\hline $2.5 \mathrm{mM}$ dNTP mix & 2.10 \\
\hline GoTaq ${ }^{\circledR}$ DNA polymerase (Promega) & 0.10 \\
\hline Final volume (deionized water) & 20.0 \\
\hline
\end{tabular}

Tail pieces from Cdh1 colony littermates were incubated with $200 \mu 1 /$ tail tip of genotyping buffer and proteinase $\mathrm{K}(200 \mu \mathrm{g} / \mathrm{ml})$ overnight at $55^{\circ} \mathrm{C}$. The tubes were flicked to resuspend the DNA and spun at 13,000 rpm for $5 \mathrm{~min}$. The supernatant was transferred to fresh tubes and precipitated with $500 \mu \mathrm{l}$ of isopropanol. The samples were spun again and the DNA pellet was resuspended in $\mathrm{H}_{2} \mathrm{O}$ and re-precipitated with 0.1 volume of $3 \mathrm{M} \mathrm{CH}_{3} \mathrm{COONa}(\mathrm{pH}$ 5.5 ) and 2.5 volumes of absolute ethanol. Following centrifugation at full speed for $10 \mathrm{~min}$, the DNA pellet was washed with $70 \%$ ethanol and resuspended in $100 \mu 1$ of $\mathrm{H}_{2} \mathrm{O} .10 \mathrm{ng}$ of 
genomic DNA was used for genotyping. Two PCR reactions were set up for each sample, one using wild-type and the other using mutant primer pairs (Appendix 2), as tabulated above.

The following program was used for the PCR:

\begin{tabular}{|c|c|c|c|}
\hline Segment & Cycles & Temperature & Time [min:sec] \\
\hline Initial denaturation & 1 & $95^{\circ} \mathrm{C}$ & $05: 00$ \\
\hline Denaturation & 30 & $95^{\circ} \mathrm{C}$ & $00: 30$ \\
\hline Annealing & & $48^{\circ} \mathrm{C}$ & $00: 45$ \\
\hline Extension & & $72^{\circ} \mathrm{C}$ & $01: 00$ \\
\hline Final extension & 1 & $72^{\circ} \mathrm{C}$ & $05: 00$ \\
\hline
\end{tabular}

The wild-type and mutant reactions were analyzed on a $1.5 \% \mathrm{w} / \mathrm{v}$ agarose gel. Agarose was boiled in $2 \mathrm{x}$ TAE to which $5 \mu \mathrm{l}$ (per $100 \mathrm{ml}$ of buffer) of ethidium bromide was added. The samples were subjected to electrophoresis at a constant voltage and the PCR fragments were visualized under a UV transilluminator. $\mathrm{Cdh} 1^{+/+}$genotype produced a single $500 \mathrm{bp}$ band in the wildtype reaction while the $\mathrm{Cdh}^{+/-}$genotype displayed a 500 bp band in the wildtype and $600 \mathrm{bp}$ band in the mutant reaction.

\subsubsection{GST-pulldown of active RhoA}

Pulldown of active RhoA was performed using recombinant GST-Rhotekin immobilized on glutathione sepharose 4B beads (GE Healthcare). Recombinant bacterial glycerol stocks of pGEX2T-GST-Rhotekin were kindly provided by Pontus Aspenstroem, Ludwig Institute for Cancer Research, Sweden. To purify the GST-tagged effectors, $300 \mathrm{ml}$ of $2 \mathrm{xYT}$ was inoculated with recombinant bacteria and bacterial growth was followed by measuring $\mathrm{OD}_{600}$. Soon after bacterial growth reached the log phase $\left(\mathrm{OD}_{600}=0.5\right)$, recombinant protein expression was induced by adding $0.5 \mathrm{mM}$ isopropyl thiogalactopyranoside (IPTG) to the culture medium. The cultures were continued to be shaken at $37^{\circ} \mathrm{C}$ until bacteria reached the decline phase $\left(\mathrm{OD}_{600}=1.6-1.8\right)$. The bacteria were spun down at $4000 \mathrm{rpm}$ for $30 \mathrm{~min}$ and the pellet was lysed in $15 \mathrm{ml}$ of ice-cold Co-IP buffer. The lysates were sonicated ( 3 bursts of $30 \mathrm{sec}$ each, $40 \%$ power) and then, centrifuged at 13,000 rpm for $30 \mathrm{~min}$ at $4^{\circ} \mathrm{C}$. Recombinant GST-tagged proteins contained in the supernatant were purified by incubating the protein lysates with glutathione sepharose beads for $4 \mathrm{~h}$ at $4^{\circ} \mathrm{C}$ followed by several washes with wash buffer $(50 \mathrm{mM}$ Tris base, $5 \mathrm{mM}$ EDTA, protease inhibitors) to remove unbound protein. To assay active RhoA in wild-type and Cdh1- 
heterozygotes, brain lysates from week 10 (W10) mice were prepared in Co-IP buffer with freshly added protease inhibitors. The lysates were incubated with GST-Rhotekin beads at $4{ }^{\circ} \mathrm{C}$ for $2 \mathrm{~h}$. Following three washes with Co-IP buffer and one wash with PBS, the beads-bound active RhoGTPases were eluted by boiling in SDS-Laemelli buffer and subjected to SDSPAGE and Western blotting.

\subsubsection{Total protein lysates}

Cortices, cerebella and hippocampi from $\mathrm{Cdh}^{+/+}$and $\mathrm{Cdh}^{+/-}$mice at P12, W14 and W16 were homogenized in Triton ${ }^{\circledR} \mathrm{X}-100$ buffer with freshly added protease inhibitors using a tissue blender. The tissue lysates were spun down and the supernatant was subjected to Bradford assay. Equal amounts of total protein were used for SDS-PAGE and Western blotting.

\subsubsection{RNA isolation, cDNA synthesis and quantitative RT-PCR}

RNA was isolated from cerebella of P12 and W16 Cdh1 $1^{+/+}$and $\mathrm{Cdh} 1^{+/-}$littermates using the phenol-chloroform extraction method. Tissue lysates were prepared in TRIzol ${ }^{\circledR}$ reagent (Life Technologies ${ }^{\mathrm{TM}}$ ). To this, 0.2 volume of chloroform was added under a fume hood and the tubes were vortexed briefly and incubated on ice for $15 \mathrm{~min}$. The samples were centrifuged at $14,000 \mathrm{rpm}$ for $15 \mathrm{~min}$ at $4^{\circ} \mathrm{C}$. The aqueous upper phase containing RNA was carefully transferred to fresh tubes avoiding the cloudy DNA interphase. RNA was precipitated with an equal volume of isopropanol and the tubes were spun down at full speed for $15 \mathrm{~min}$ at $4^{\circ} \mathrm{C}$. The supernatant was discarded and the pellet was washed with $1 \mathrm{ml}$ of $70 \%$ ethanol (prepared in freshly autoclaved water). After centrifuging at 14,000 rpm for $10 \mathrm{~min}$, the pellet was airdried at RT and resuspended in $100 \mu 1$ of autoclaved water. RNA concentration was measured $\left(\mathrm{A}_{260} / \mathrm{A}_{280}\right)$ and the samples were diluted to $100 \mathrm{ng} / \mu 1$ for $\mathrm{cDNA}$ synthesis.

cDNA synthesis was performed using SuperScript ${ }^{\mathrm{TM}}$ III First-Strand Synthesis System (Life Technologies ${ }^{\mathrm{TM}}$ ) according to manufacturer's instructions. $10 \mu 1$ of RNA template was used and the reaction was primed by oligo(dT) $)_{20}$ and random hexamers.

For quantitative RT-PCR, primers for mouse Smurfl were designed using PrimerBLAST (Appendix 2) Primer sequences to internal controls Gapdh (Oligo ID \#14539 and $\# 14540$ ) and $\beta$-actin (Oligo ID \#9146 and \#9147) were retrieved from the primer database, AGCT Lab, MPI-EM, Göttingen. The cDNA samples were diluted 1:10 in water. For ten reactions, the master mix was prepared as shown below: 


\begin{tabular}{lc}
\hline Component & Volume $(\mu \mathrm{l})$ \\
\hline \hline SYBR Green (Applied Biosystems) & 50.00 \\
\hline $10 \mu \mathrm{M}$ sense primer & 0.10 \\
\hline $10 \mu \mathrm{M}$ antisense primer & 0.10 \\
\hline $\mathrm{H}_{2} \mathrm{O}$ & 10.00 \\
\hline
\end{tabular}

$4 \mu 1$ of the cDNA sample was pipetted into the wells of a 96-well plate, and then $6 \mu 1$ of the master mix was added. The sample was mixed by pipetting. Each condition was represented in triplicates and reactions were set up using Smurfl primers and separately using primers for Gapdh or $\beta$-actin internal controls. For each target gene, a non-template control was included. The plate was spun briefly at $1400 \mathrm{rpm}$ and subjected to quantitative RT-PCR using Light Cycler R480 (Roche Applied Science, Mannheim). The Light Cycler R480 Relative Quantification Software was used to run the following program:

\begin{tabular}{llll}
\hline \multicolumn{1}{c}{ Segment } & Cycles & Temperature & Time [min:sec] \\
\hline \hline Denaturation & 1 & $95^{\circ} \mathrm{C}$ & $05: 00$ \\
\hline Amplification & 30 & $95^{\circ} \mathrm{C}$ & $00: 10$ \\
\cline { 3 - 4 } & & $60^{\circ} \mathrm{C}$ & $00: 30$ \\
\cline { 2 - 4 } & & $72^{\circ} \mathrm{C}$ & $00: 01$ \\
\hline Cooling & 1 & $40^{\circ} \mathrm{C}$ & $00: 30$ \\
\hline
\end{tabular}

Results were analyzed using the 'absolute quantification' method and the threshold cycle $[\mathrm{C}(\mathrm{t})]$ values were exported as an excel file. The $\Delta \Delta \mathrm{C}(\mathrm{t})$ method was used whereby, Smurf1 expression was normalized to that of Gapdh or $\beta$-actin and mutant expression was further normalized to the control group. Expression was calculated as the 'delog' of the $\Delta \Delta \mathrm{C}(\mathrm{t})$ value.

$$
\text { Relative mRNA levels }=\frac{\left(\frac{\text { mRNA levels of target gene }}{\text { mRNA levels of internal control }}\right)}{\text { mean of wildtype }\left(\frac{\text { mRNA levels of target gene }}{\text { mRNA levels of internal control }}\right)}
$$




\subsection{In vivo experiments}

\subsubsection{In vivo electroporation of cerebella of postnatal rat pups}

In vivo electroporation was performed as described previously (Konishi et al., 2004). A plasmid DNA-containing solution in PBS (4 $\mu \mathrm{l} /$ animal) together with $0.3 \%$ fast green was injected into the cerebellar cortex of P4 rat pups using a $25 \mu \mathrm{l}$ syringe (Hamilton, Bonaduz, Switzerland) with a 30 -guage needle. $16 \mu \mathrm{g}$ of the U6-CMV-EGFP or p250GAP RNAi/Smurf1 RNAi-CMV-EGFP plasmid and $4 \mu \mathrm{g}$ Bcl- $\mathrm{x}_{\mathrm{L}}$ expression plasmid were injected per animal. Following DNA injection, animals were subjected to repeated electric pulses (5 pulses of 155$170 \mathrm{~V}$ for $50 \mathrm{~ms}$ with intervals of $950 \mathrm{~ms}$ ) and allowed to recover on a heating pad. Electroporated pups were returned to the mother until P9.

\subsubsection{Isolation of cerebella, fixation and immunohistochemistry on cryo-sections}

At P9, the electroporated pups were sacrificed and the cerebella were collected in HHGN dissection medium. GFP-positive cerebella were washed with PBS and fixed in 4\% PFA by rocking overnight at $4^{\circ} \mathrm{C}$. They were again washed with PBS and submerged in $30 \%$ $\mathrm{w} / \mathrm{v}$ sucrose in PBS and kept upright at $4^{\circ} \mathrm{C}$ for 3-4 $\mathrm{h}$ until the cerebella sank to the bottom. The cerebella were washed again and embedded in plastic moulds (on dry ice) in embedding medium, consisting of equal volumes of OCT (Tissue Tek, Sakura, Torrance, CA) and 30\% sucrose in PBS. The embedded cerebella were frozen at $-80^{\circ} \mathrm{C}$.

$40 \mu \mathrm{m}$ coronal sections of cerebella were prepared in a cryostat (Leica). Sections were collected on poly-lysine coated glass slides (Macherey-Nagel). Slides were allowed to dry and then stored at $-80^{\circ} \mathrm{C}$ until immunostaining.

Prior to immunostaining, frozen slides were briefly thawed. All steps were performed at RT unless stated otherwise. Sections were washed twice with PBS, each time for $10 \mathrm{~min}$, by rocking. For blocking, the slides were incubated with blocking buffer containing $10 \%$ NGS, $2 \%$ BSA, $0.5 \%$ Triton $^{\circledR}$ X-100 in PBS for 30 min. Primary antibody, mouse monoclonal antiGFP (Santa Cruz), was diluted 1:200 in blocking buffer, added to the slides and incubated overnight at $4{ }^{\circ} \mathrm{C}$. On the next day, the slides were washed three times with PBS on a rocker, 10 min per wash. The sections were then incubated for $2 \mathrm{~h}$ with Cy2-conjugated anti-mouse antibody (Dianova) diluted 1:1000 also in blocking buffer. The slides were washed three times with PBS. After counterstaining with Hoechst for $5 \mathrm{~min}$, sections were again washed twice with PBS. The slides were dried briefly, mounting medium was applied onto the slides and the 
sections were covered by carefully placing glass cover slips over the mounting medium. Images of GFP-positive neurons were captured by a blinded observer using the green channel, with a $20 x$ or $10 x$ objective.

\subsubsection{Axon growth and migration assays}

Migration and morphological assays in vivo were performed as described before (Konishi et al., 2004; Stegmuller et al., 2006). The location of transfected neurons in the cerebellum was assessed as either external granule layer (EGL)/molecular layer (ML) or internal granule layer (IGL). Since the ascending axon cannot always be visualized owing to technical challenges (sectioning), the parallel fiber index (correlation of granule neurons to parallel fibers) was determined by counting the number of cells and parallel fibers independently in a set of sections. Typically, a correlation of about $90 \%$ was obtained.

Additionally, axon morphology was examined and neurons were scored based on whether they had long and thin parallel fibers as in the control condition, or short and thick processes. For arbitrary quantification of axonal lengths, the axons in the control condition were measured as long as they could be traced in a given section. This was assumed as the minimum average length of control axons. Shorter axons in the test conditions however, were measured to their entirety.

Statistical analyses (ANOVA) were performed using the Graph Pad Prism 5.0c software. The mean values were plotted and error bars represent mean +SEM. 


\section{Results}

\subsection{Growth-promoting signaling programs antagonize Cdh1-APC- inhibition of axon growth}

Neuronal morphogenesis is tightly regulated by extrinsic factors including growth factors, guidance cues and extracellular matrix molecules. Neurotrophin binding to Trk receptors activates the receptor kinase domain which in turn, facilitates docking of adaptor proteins to the activated receptor (Huang and Reichardt, 2003; Kaplan et al., 1991b). This enables interplay between neurotrophin signaling and intracellular signaling cascades which comprise Ras/extracellular regulated kinase (ERK, also called mitogen activated protein kinase or MAPK), phosphotidylinositol 3-kinase (PI3K)/Akt and downstream pathways involving the small Rho GTPases (Patapoutian and Reichardt, 2001). Activated PI3K and MAPK are associated with axon growth stimulating programs downstream of neurotrophin signaling (Huang and Reichardt, 2001). Notably, the neurotrophin receptor TrkB enhances axon growth in sympathetic neurons by activating MAPK and PI3K signaling by virtue of its She domain (Atwal et al., 2000). Whether intrinsic Cdh1-APC pathway intercalates with extrinsic neurotrophin signaling in axonal morphogenesis was the first question that I sought to address in my study. Thus, I systematically searched for potential cross-talks between axon growth stimulating cascades involving PI3K and MAPK and the Cdh1-APC pathway in a set of functional assays.

\subsubsection{Inhibition of PI3-kinase signaling inhibits Cdh1 knockdown-induced axonal growth in vitro}

To test for a possible cross-talk between the PI3K signaling pathway and Cdh1-APC regulated axon growth control, I first assessed the levels of phospho-Akt, a downstream effector of PI3K in neuronal lysates from wild-type $\mathrm{Cdh}^{+/+}$and $\mathrm{Cdh} 1$-heterozygous $\mathrm{Cdh}^{+/-}$ mice. Owing to their embryonic lethality, the $\mathrm{Cdh}^{-/}{ }^{-/}$mice were not available for analyses ( $\mathrm{Li}$ et al., 2008). Lysates of cerebellar granule neurons isolated from postnatal day 5 (P5) wild-type and Cdh1-heterozygous mice were immunoblotted with antibodies against $\mathrm{Cdh} 1$ and SnoN, the latter is a known target of Cdh1-APC (Stegmuller et al., 2006; Stroschein et al., 2001; Wan et al., 2001). Expectedly, I found a decrease in Cdh1 and an increase in SnoN protein in the $\mathrm{Cdh}^{+/}$as compared to wild-type mice. Next, the lysates were probed for total and phospho- 
Akt. Global levels of neither phospho-Akt nor total Akt are affected in the Cdh1 heterozygotes as compared to wild-type (Figure 3.1).
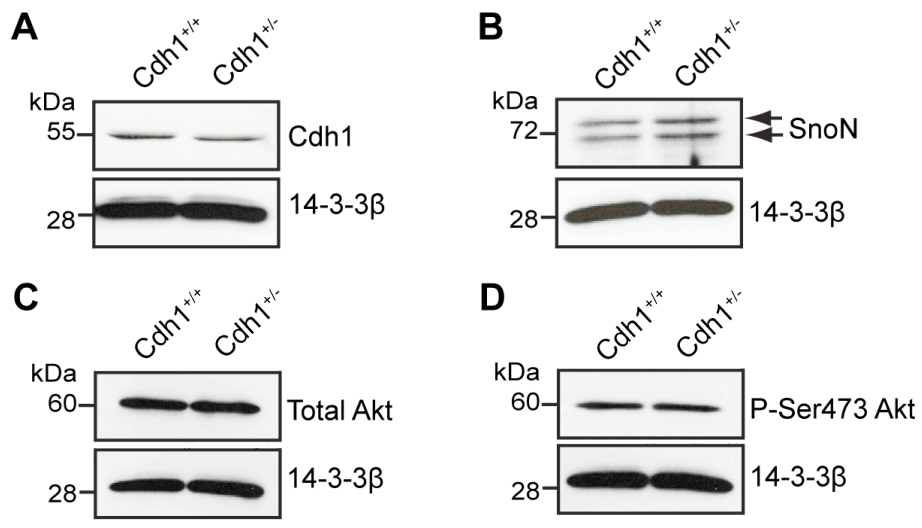

Figure 3.1 Global PI3K activity is not affected in $\mathrm{Cdh1}^{+/-}$mice. Lysates of cultured granule neurons from $\mathrm{Cdh}^{+/+}$and $\mathrm{Cdh}^{+/-}$mice were subjected to immunoblotting with A. Cdh1, B. SnoN, C. total Akt or D. phosphoSer473 Akt antibody. 14-3-3 $\beta$ served as the loading control.

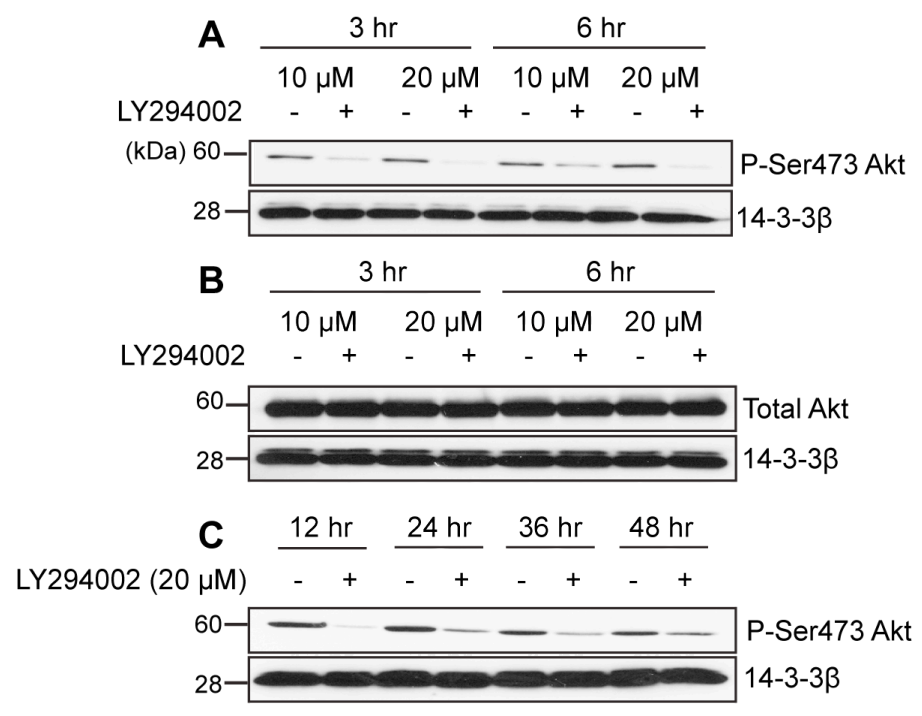

Figure 3.2 Pharmacological inhibition of PI3K pathway. Lysates of cerebellar granule neurons treated with 10 or $20 \mu \mathrm{M}$ LY294002 for 3 and $6 \mathrm{~h}$ were subjected to immunoblotting using A. phospho-Ser473 Akt or B. total Akt antibody. 14-3-3 $\beta$ served as the loading control. C. Lysates of granule neurons treated with $20 \mu \mathrm{M}$ LY294002 for 12, 24, 36 and $48 \mathrm{~h}$ were immunoblotted using the phospho-Ser473 Akt and 14-3-3 $\beta$ antibodies.

To test whether PI3K signaling is active under Cdh1 knockdown in vitro, I adopted a pharmacological approach. LY294002 (LY) is a reversible inhibitor of PI3K. Different concentrations of the drug were tested on cultured cerebellar granule neurons and PI3K activity was assessed by probing for phosphorylated Akt (Figure 3.2). $20 \mu \mathrm{M}$ LY effectively inhibits PI3K activity and hence, results in a reduced phosphorylation of Akt in granule neurons. 


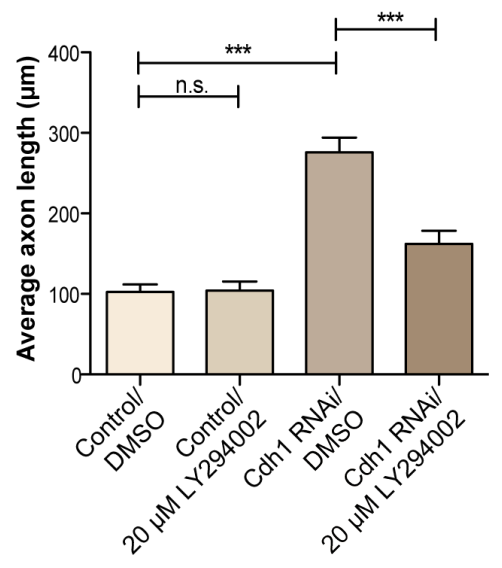

Figure 3.3 Inhibition of PI3K signaling affects Cdh1 knockdown-induced axon growth. Cerebellar granule neurons were transfected $8 \mathrm{~h}$ after plating (day in vitro $0=$ DIV 0) with control vector U6 or the Cdh1 RNAi plasmid together with GFP and $\mathrm{BCL}_{\mathrm{XL}}$ plasmids and maintained in $\mathrm{BME}+$ insulin. At DIV 1, neurons were treated with $20 \mu \mathrm{M}$ LY294002 or vehicle for $48 \mathrm{~h}$. At DIV 3, neurons were subjected to immunocytochemistry using the GFP antibody. Axonal length was measured in GFP-positive transfected neurons using ImageJ software. A total of 292 neurons was measured (ANOVA, $* * * \mathrm{p}<0.0001$, n.s. $=$ not significant, values indicate mean + SEM).

Next, granule neurons were transfected at day in vitro 0 (DIV 0) with a control vector or Cdh1 RNAi plasmid together with plasmids encoding GFP and $B C L_{X L}$, a pro-survival protein. The neurons were treated with $20 \mu \mathrm{M}$ LY or vehicle at DIV 1 for 48 hours, then fixed and immunostained with the GFP antibody. In axon growth assays, Cdh1 knockdown causes a significant increase in axon length as compared to control (Konishi et al., 2004). While vehicle has no effect on Cdh1 knockdown enhanced axon growth, I observed a significant decrease in axonal length in Cdh1 knockdown granule neurons upon treatment with the PI3K inhibitor, LY (Figure 3.3) suggesting that the Cdh1 knockdown axon phenotype is modulated by PI3K signaling. Taken together, the results implicate active PI3K signaling in axonal growth associated with Cdh1 knockdown in vitro.

\subsubsection{Inhibition of MAP-kinase signaling significantly reduces axon length in Cdh1 knockdown neurons}

To test whether MAPK activity is induced under Cdh1 deficiency, I assessed the levels of phospho-Erk1/2 in neuronal lysates from $\mathrm{Cdh}^{+/+}$and $\mathrm{Cdh}^{+/-}$mice. Erk1/2 is a MEK effector that is phosphorylated upon activation of the MAPK cascade. Granule neuron lysates were probed for total and phospho-Erk1/2. Granule neurons from $\mathrm{Cdh}^{+/-}$mice exhibit a consistent increase in phospho but not total Erk1/2 levels as compared to those of wild-type littermates (Figure 3.4). In a drug-based morphological assay, I examined MAPK activity in Cdh1 knockdown neurons. Transfected neurons treated with vehicle or U0126, a highly selective inhibitor of MEK1/2, were analysed for axonal lengths. The results of the assays 
showed no effect of the drug on axonal lengths in control neurons but a significant decrease in axon length in Cdh1 knockdown granule neurons treated with the MEK inhibitor as compared to vehicle treatment (Figures 3.5 and 3.6). Taken together, the results implicate MAPK signaling in Cdh1-regulated axonal growth.
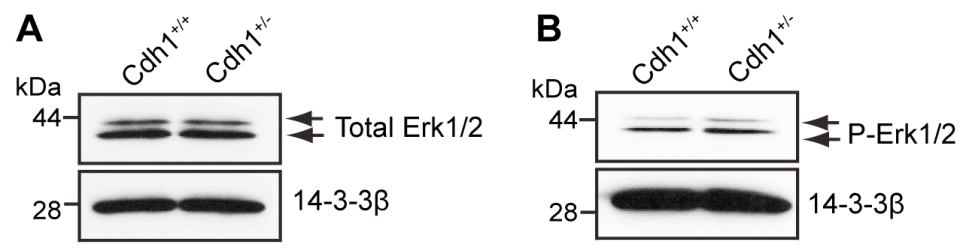

Figure 3.4 Phospho-Erk is induced in $\mathrm{Cdh}^{+/-}$mice. Lysates of cultured granule neurons from $\mathrm{Cdh} 1^{+/+}$and $\mathrm{Cdh}^{+/-}$mice were subjected to immunoblotting with A. phospho or B. total Erk1/2 antibody. 14-3-3 $\beta$ served as the loading control.

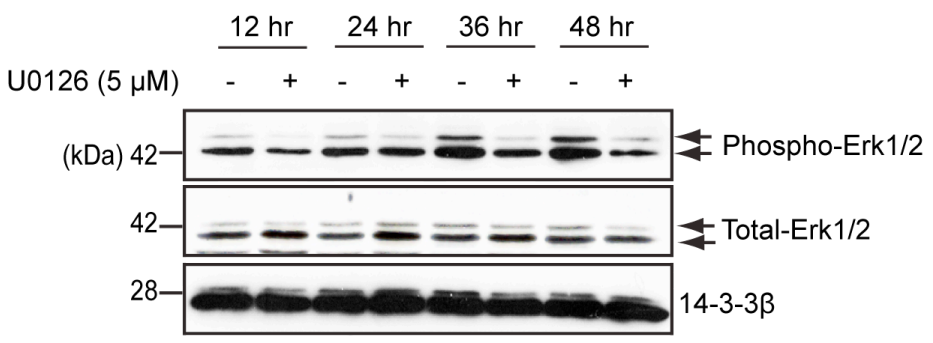

Figure 3.5 Pharmacological inhibition of MAPK signaling. Lysates of cerebellar granule neurons treated with 5 $\mu \mathrm{M} \mathrm{U} 0126$ for $12,24,36$ and $48 \mathrm{~h}$ were subjected to immunoblotting using phospho or total Erk1/2 antibodies. $14-3-3 \beta$ served as the loading control.

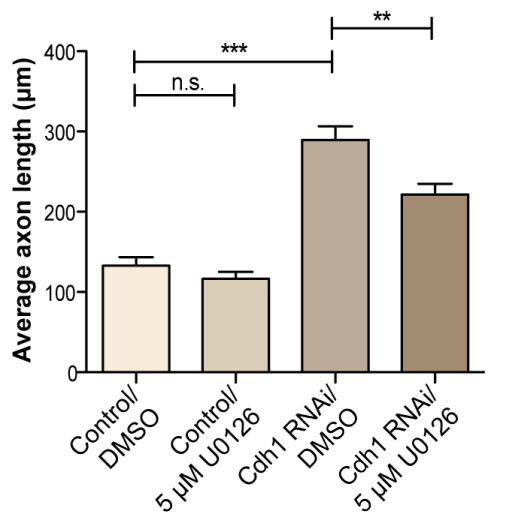

Figure 3.6 MAPK inhibition reduces axon growth under Cdh1 knockdown. Granule neurons were transfected at DIV 0 with control vector U6 or the Cdh1 RNAi plasmid as described in Figure 3.3. At DIV 1, neurons were treated with $5 \mu \mathrm{M}$ U0126 or vehicle for $48 \mathrm{~h}$. At DIV 3, neurons were analyzed as in Figure 3.3. A total of 445 neurons was measured (ANOVA, ${ }^{* * *} \mathrm{p}<0.0001, * * \mathrm{p}<0.05$, n.s. $=$ not significant, values indicate mean + SEM). 


\subsection{Cdh1-APC exerts control on axon growth by stimulating growth- inhibitory cytoskeletal Rho GTPases}

Neurite outgrowth and axon extension require a dynamic cytoskeleton. Rho GTPases have emerged as key players in cytoskeletal reorganization and microtubule orientation and stabilization, which are the major events that occur during neuritogenesis and growth cone steering (Dickson, 2001; Govek et al., 2005). This prompted me to investigate the role of the Rho GTPases RhoA, Rac1 and Cdc42 in Cdh1-APC-mediated axon growth suppression.

\subsubsection{RhoA activity reverses Cdh1 knockdown-stimulated axon growth}
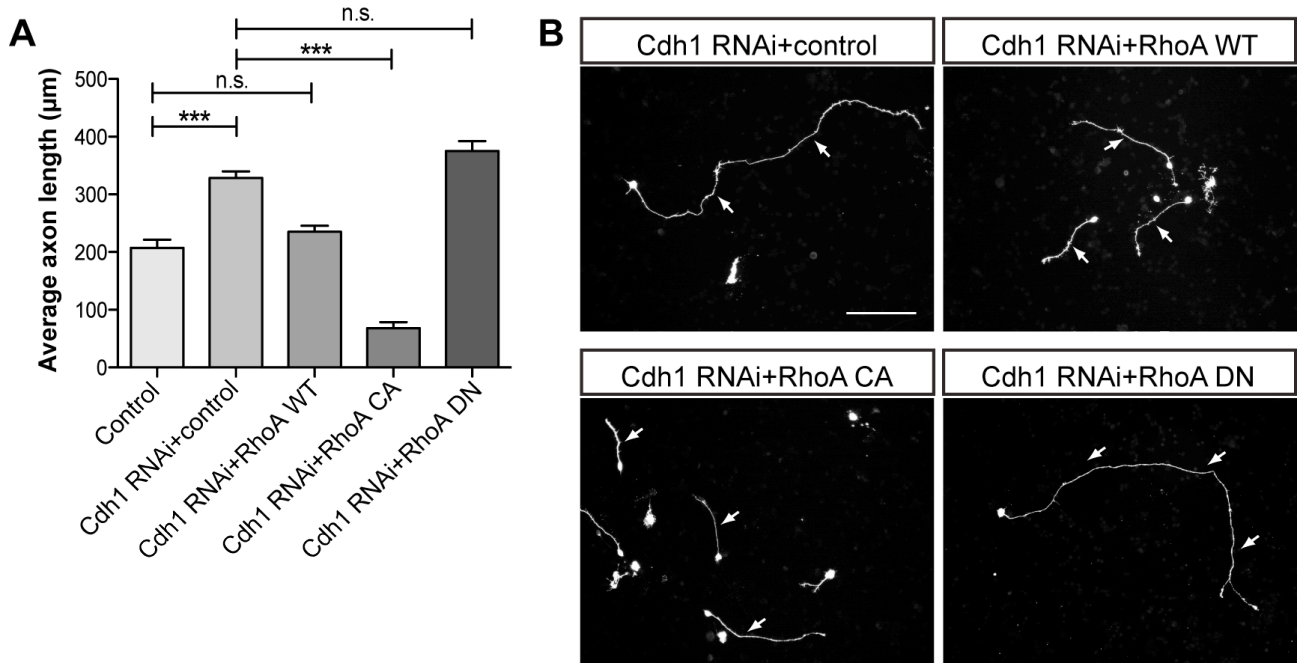

Figure 3.7 RhoA inhibits Cdh1 knockdown-induced axon growth. A. Granule neurons were transfected at DIV 0 with control vector U6 or the Cdh1 RNAi plasmid together with empty vector pCEFL, the plasmid expressing wild-type RhoA (RhoA WT), constitutively active RhoA (RhoA CA) or dominant negative RhoA (RhoA DN). At DIV 3, neurons were analyzed as in Figure 3.3. A total of 443 neurons was measured (ANOVA, $* * * p<0.0001$, n.s. $=$ not significant, values indicate mean + SEM). B. Representative images of transfected neurons in A. Scale bar equals $100 \mu \mathrm{m}$. Arrows indicate axons.

To study the role of active RhoA in Cdh1 knockdown-mediated axon elongation, granule neurons were transfected at DIV 0 with the control vector, or the Cdh1 RNAi plasmid together with the empty vector, wild-type (WT), constitutively active (CA) or dominant negative (DN) forms of RhoA (Coso et al., 1995). Again, Cdh1-knockdown enhances axon length as compared to control neurons. However, co-expression of RhoA WT significantly reduces axonal length in the Cdh1 knockdown neurons and rescues the phenotype to control levels. This effect is further accentuated by RhoA CA while the expression of RhoA DN has little or no effect on axon growth under Cdh1-knockdown (Figure 3.7).

In further axon growth assays, the effects of the aforementioned versions of RhoA on axon growth were examined. This experiment (Figure 3.8) was performed by Shih-Ju Lee, 
Stegmüller Lab, Max Planck Experimental Medicine (MPI-EM), Göttingen. While RhoA WT in Cdh1 knockdown neurons counteracts the long-axon phenotype and restores axon length to control levels, RhoA WT on its own has no significant effect on axon growth (Figure 3.8). Similarly, RhoA DN has no effect on axon growth in Cdh1 knockdown neurons which already display the long-axon phenotype, but on its own, RhoA DN is strongly growth-promoting, suggesting that the effect of RhoA WT or DN expression does not add to the Cdh1 knockdown phenotype but instead, that RhoA operates downstream of Cdh1-APC in a linear pathway in the control of granule neuron axon outgrowth. Taken together, the results suggest that RhoA activity controls axon length downstream of Cdh1-APC.

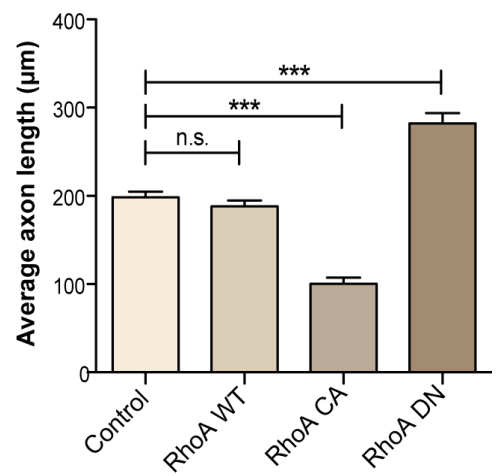

Figure 3.8 RhoA is axon growth-inhibiting. Granule neurons were transfected at DIV 0 with control vector pCEFL or plasmids encoding RhoA WT, RhoA CA or RhoA DN. At DIV 3, neurons were analyzed as in Figure 3.3. A total of 717 neurons was measured (ANOVA, $* * * p<0.0001$, n.s. $=$ not significant, values indicate mean +SEM). The experiment was performed by Shih-Ju Lee, Stegmüller Lab, MPI-EM, Göttingen.

\subsubsection{Interfering with Rac1 activity impairs axon length in Cdh1 knockdown neurons}
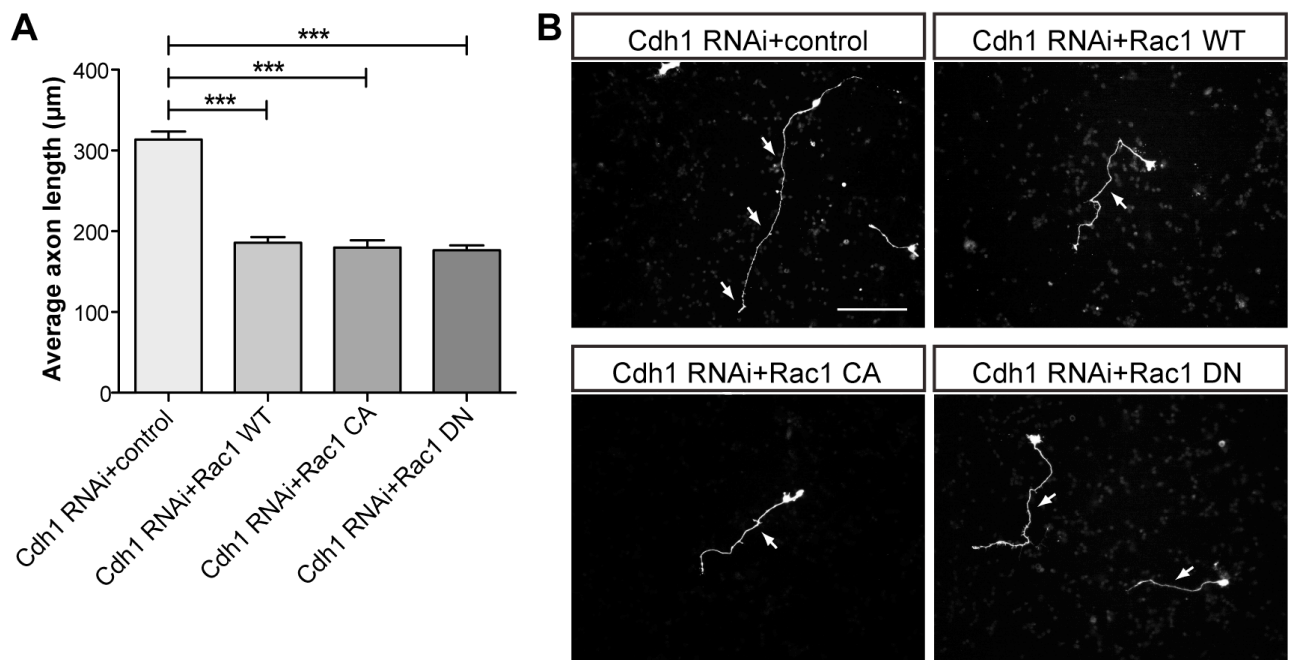

Figure 3.9 Rac1 affects Cdh1 knockdown-induced axon growth. Granule neurons were transfected at DIV 0 with the Cdh1 RNAi plasmid together with empty vector pCEFL, the plasmid expressing wild-type Rac1 (Rac1 WT), constitutively active Rac1 (Rac1 CA) or dominant negative Rac1 (Rac1 DN). At DIV 3, neurons were analyzed as in Figure 3.3. A total of 760 neurons was measured (ANOVA, $* * * p<0.0001$, values indicate mean + SEM). 
To determine whether Rac1 GTPase acts in concert with Cdh1 knockdown in axon growth suppression, I carried out a similar experiment as described in section 3.2.1. Expression of wild-type (Rac1 WT), constitutively active (Rac1 CA) or dominant negative (Rac1 DN) forms of Rac1 together with Cdh1 RNAi plasmid results in a significant decrease in axon length in all three conditions as compared to control Cdh1 knockdown neurons (Figure 3.9). The result is consistent with other studies (Kuhn et al., 1998; Luo et al., 1996), which reported axon growth defects whenever Rac1 activity is manipulated.

\subsubsection{Active Cdc42 significantly reduces axon length under Cdh1 knockdown conditions}

A

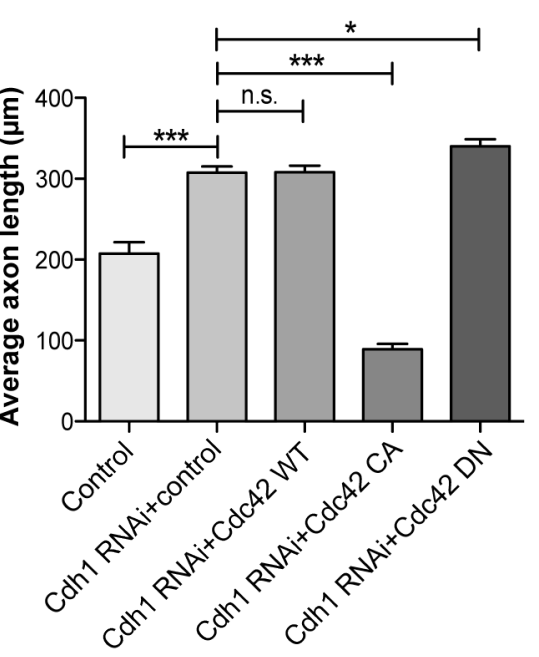

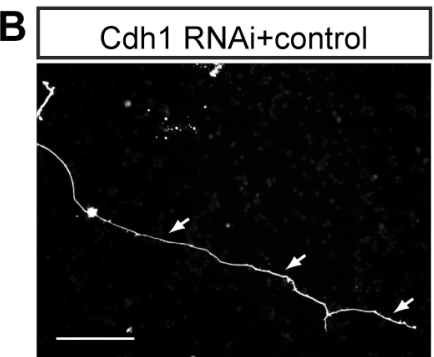

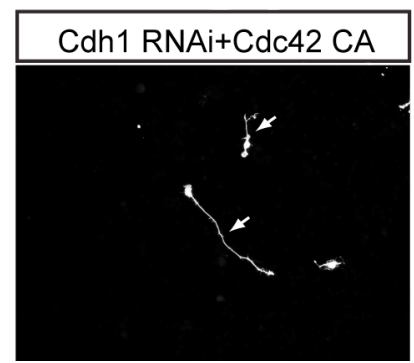

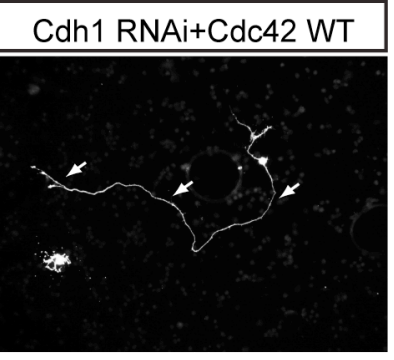

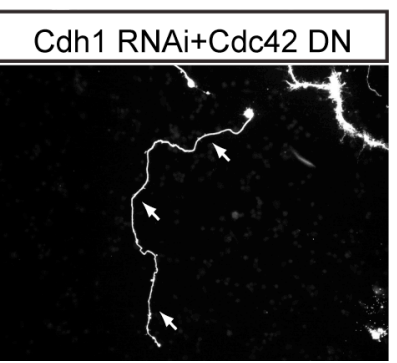

Figure 3.10 Cdc42 activity inhibits Cdh1 knockdown-induced axon growth. A. Granule neurons were transfected at DIV 0 with control vector U6 or the Cdh1 RNAi plasmid together with empty vector pCEFL, the plasmid expressing wild-type Cdc42 (Cdc42 WT), constitutively active Cdc42 (Cdc42 CA) or dominant negative Cdc42 (Cdc42 DN). At DIV 3, neurons were analyzed as in Figure 3.3. A total of 1538 neurons was measured $\left(\right.$ ANOVA, $* * * p<0.0001,{ }^{*} p<0.05$, n.s. $=$ not significant, values indicate mean + SEM). B. Representative images of transfected neurons in A. Scale bar equals $100 \mu \mathrm{m}$. Arrows indicate axons. The experiment was reproduced by Jan Hoeber, Bachelor's student, Stegmüller Lab, MPI-EM, Göttingen.

Next, granule neurons were transfected with control, or Cdh1 RNAi plasmid together with empty vector, wild-type (WT), constitutively active (CA) or dominant negative (DN) Cdc42 expression plasmids. While Cdc42 CA counteracts the Cdh1 knockdown induced axon elongation, Cdc42 WT displays no and Cdc42 DN a slightly enhancing effect on axon growth (Figure 3.10). The data indicate that active Cdc42 annuls the Cdh1 knockdown phenotype while inactive Cdc42 has a growth-promoting effect. Cdc42 WT shows little effect on axon growth suggestive of active Rho GTPase Activating Proteins (RhoGAPs) present in granule neurons that rapidly inactivate Rho GTPases overexpressed in the neurons. 


\subsection{Cdh1 interacts with Smurf1, a HECT ubiquitin ligase that degrades RhoA, in the control of axon growth}

The finding that Cdh1 knockdown overrides myelin inhibition of axon growth (Konishi et al., 2004) together with my preliminary analyses of growth-promoting and growth-inhibitory pathways that potentially intersect the Cdh1-APC pathway of axon growth regulation raised the possibility of a cross-talk between RhoA signaling and Cdh1-APC-regulated axon growth. RhoA has been extensively studied as a key downstream component of myelin signaling (McGee and Strittmatter, 2003; Schwab, 2004; Yiu and He, 2006). However given that RhoA operates downstream of Cdh1 in axon growth control and that both proteins have an inhibitory effect on axon growth, I reasoned that RhoA is unlikely to be a direct target of the E3 ligase, Cdh1-APC. Hence, I adopted a candidate approach to identify proteins that negatively regulate RhoA, including the RhoA GAPs and the E3 ligase Smurf1 to identify a novel Cdh1 substrate that may act in the Cdh1-APC/RhoA pathway of axon growth regulation.

\subsubsection{Smurf1, a brain-abundant HECT ligase, is a novel interactor of Cdh1}
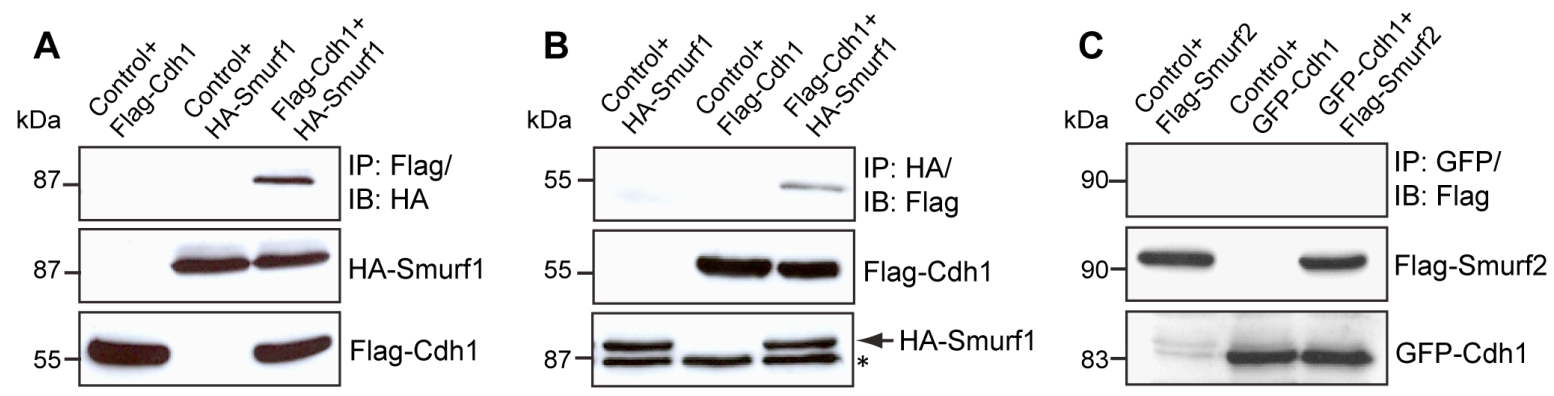

Figure 3.11 Smurf1 interacts with Cdh1 in heterologous cells. A. Lysates of 293T cells transfected with empty vector pCMV5 and Flag-Cdh1 or HA-Smurf1 expression plasmids, or both Flag-Cdh1 and HA-Smurf1 plasmids were subjected to immunoprecipitation with the Flag antibody and immunoblotting with the HA antibody. B. Reciprocal co-immunoprecipitation of A. Arrow and asterisk indicate HA-Smurfl and non-specific bands, respectively. C. Lysates of 293 T cells transfected with GFP and Flag-Smurf2 expression plasmids, GFP-Cdh1 plasmid and empty vector pCMV5, or both GFP-Cdh1 and Flag-Smurf2 plasmids were subjected to immunoprecipitation with the GFP antibody followed by immunoblotting with the Flag antibody.

Smurf1, a homologous to E6-AP C-terminus (HECT) E3 ubiquitin ligase, is wellacclaimed for targeting RhoA for proteasomal degradation (Wang et al., 2003). In addition, recent reports have identified a role for Smurf1 in promoting neurite outgrowth and axon initiation (Bryan et al., 2005; Cheng et al., 2011). I hence asked whether Smurfl takes part in the Cdh1-APC/RhoA pathway of control of axonal morphogenesis.

First, I explored a potential interaction of exogenous Smurf1 and Cdh1 in 293T cells by coimmunoprecipitation assays. Lysates of $293 \mathrm{~T}$ cells transfected with control or HA-Smurf1 
and Flag-Cdh1 expression plasmids were subjected to immunoprecipitation with the HA antibody followed by immunoblotting with Flag antibody, or vice versa. Additionally, I tested the interaction between Flag-tagged Smurf2, a Smurf1 homolog and GFP-Cdh1. I found that Cdh1 specifically interacts with Smurf1 but not with Smurf2 (Figure 3.11). To demonstrate the interaction in vivo, I first established that a Cdh1 polyclonal antibody immunoprecipitates Cdh1 together with the APC subunit Cdc27 (Figure 3.12A). The polyclonal antibody or preimmune serum was then used in co-immunoprecipitation analysis to precipitate $\mathrm{Cdh} 1$ from mouse brain lysates, followed by immunoblotting with the Smurfl antibody. Smurf1 associates with Cdh1 in the mouse brain (Figure 3.12B). These experiments identify the E3 ligase Smurf1 as a novel interactor of Cdh1.

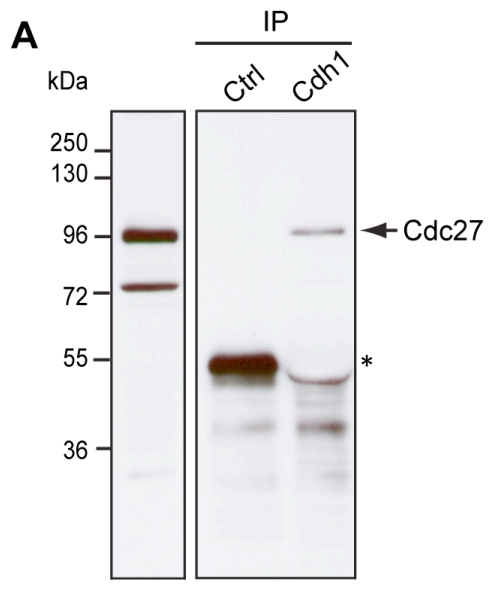

IB: Cdc27

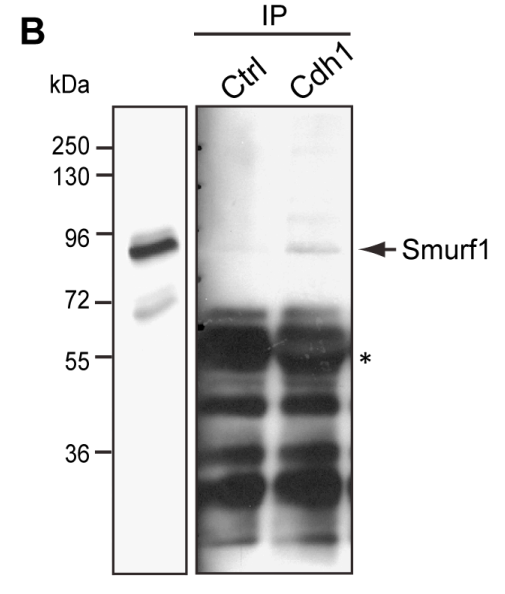

IB: Smurf1

Figure 3.12 Smurf1 interacts with Cdh1 in the mouse brain. P9 mouse brain lysates were subjected to immunoprecipitation using control serum or the Cdh1 antibody followed by immunoblotting with A. Cdc 27 or B. Smurf1 antibody. Arrow indicates Cdc27 or Smurf1 and asterisk indicates $\operatorname{IgG}_{\mathrm{H}}$.

Next, Smurfl expression was evaluated in brain and non-neuronal tissues isolated from P20 mouse. Tissue lysates were immunoblotted with the Smurf1 antibody and the internal control 14-3-3 $\beta$. Smurf1 expression is abundant in the cortex, cerebellum and hippocampus (Figure 3.13A). The temporal expression pattern of Smurf1 was assessed in brain tissues from rats at different ages and high levels of Smurf1 expression are noticeable in early development followed by a steep decrease in protein levels in later postnatal ages (P14-P30) and adulthood (Figure 3.13B-D). Smurf1 expression pattern is suggestive of a role for Smurf1 in dynamic growth promoting events in early development. 

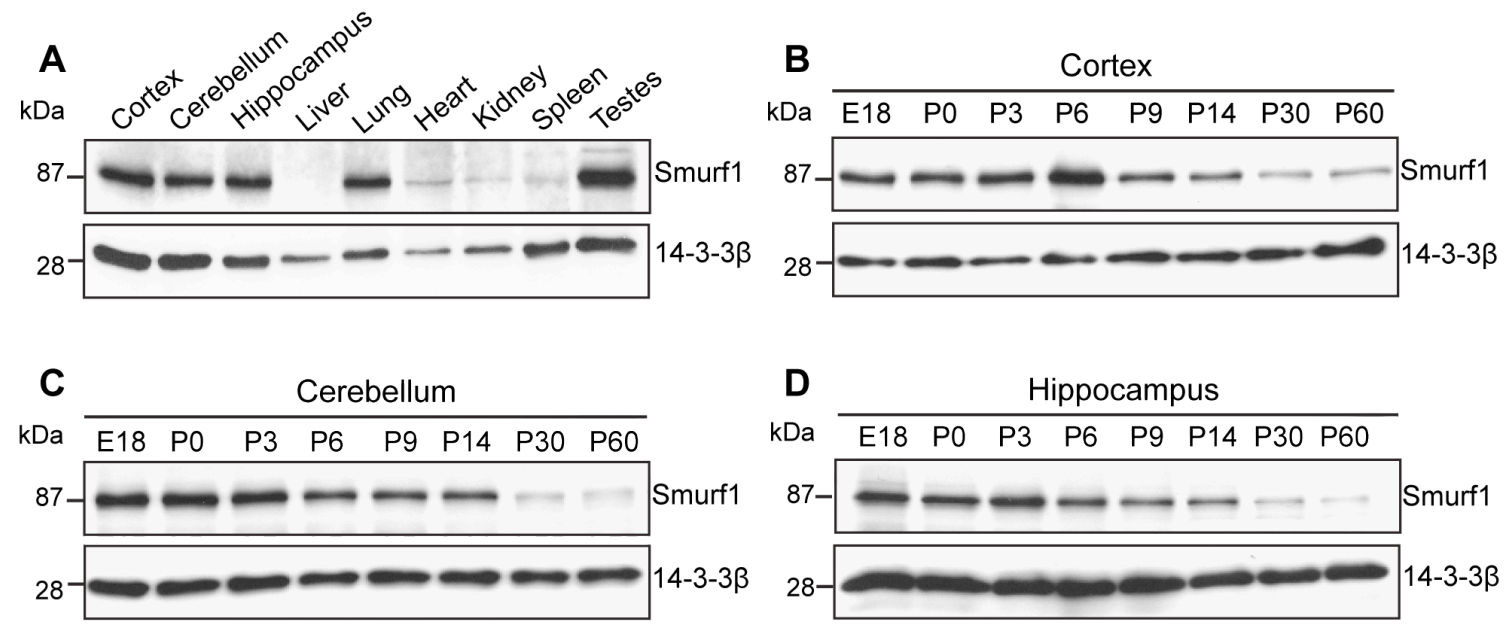

Figure 3.13 Smurf1 is brain-abundant. A. Lysates of indicated tissues collected from wild type P20 mouse were subjected to immunoblotting using the Smurf1 antibody. 14-3-3 $\beta$ served as loading control. B. Cortices, C. Cerebella or D. Hippocampi were collected from wild type rats at the indicated days and lysates were subjected to immunoblotting using the Smurf1 antibody. 14-3-3 $\beta$ served as loading control. $\mathrm{E}=$ embryonic, $\mathrm{P}=$ postnatal.

A
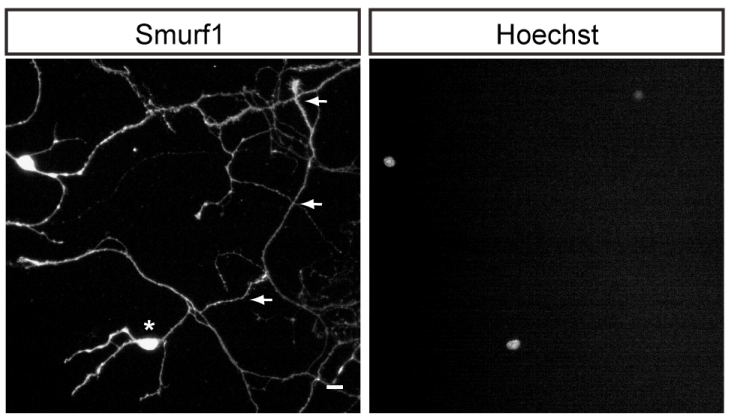

B

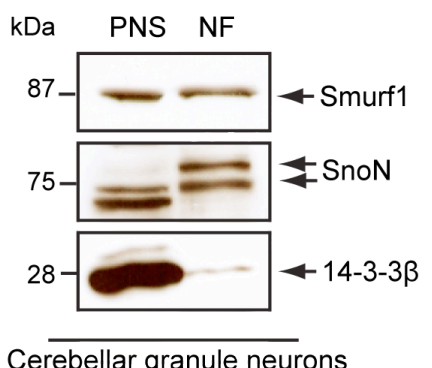

C

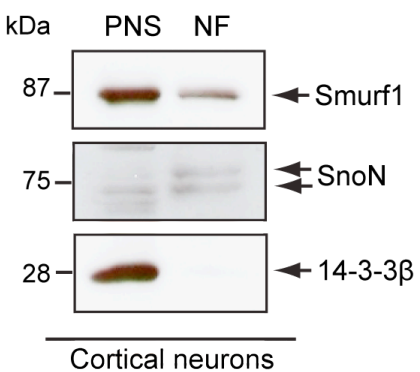

Figure 3.14 Smurf1 is a nucleocytoplasmic protein. A. Granule neurons at DIV 2 were subjected to immunocytochemistry with the Smurfl antibody and the nuclear dye bisbenzimide Hoechst 33258. Arrows and asterisk indicate axons and soma, respectively. Scale bar equals $10 \mu \mathrm{m}$. B. Cerebellar granule neurons or C. cortical neurons were subjected to subcellular fractionation. Nuclear (NF) and postnuclear supernatant (PNS) fractions were immunoblotted using Smurf1, SnoN and 14-3-3 $\beta$ antibodies. SnoN and 14-3-3 $\beta$ served as controls for NF and PNS, respectively. Subcellular fractionation was performed by Dr. Judith Stegmüller, MPI-EM, Göttingen.

To identify the subcellular localization of Smurf1, cultured granule neurons were subjected to immunocytochemistry at DIV 2 using a mouse monoclonal Smurfl antibody. Smurf1 expression is evident in the soma, cytoplasm and all along the processes (Figure 3.14A). In addition, cerebellar granule neurons and cortical neurons were subjected to 
subcellular fractionation analysis. Subcellular fractionation was performed by Dr. Judith Stegmüller, MPI-EM, Göttingen. Neuronal lysates were grossly fractionated into postnuclear and nuclear fractions each of which was immunoblotted with the Smurf1, SnoN and 14-3-3 $\beta$ antibodies. The latter two served as positive controls for nuclear and cytoplasmic fractions, respectively. Smurfl is present both in nuclear and postnuclear supernatant fractions (Figure 3.14B, C). The results concur with previous findings that Smurf1 binds Smads and shuttles between the nucleus and cytoplasm to regulate TGF- $\beta$ signaling (Inoue and Imamura, 2008; Tajima et al., 2003).

\subsubsection{Smurf1 is degraded in a proteasome-dependent manner and its protein levels are induced in Cdh1-heterozygotes}

Smurf1 interacts with the E3 ligase Cdh1-APC and is thus a potential substrate of Cdh1-APC destined for degradation. First, I asked if Smurf1 is degraded in a proteasomedependent manner. To test this, granule neurons were treated with lactacystin, a selective inhibitor of the $26 \mathrm{~S}$ proteasome, or vehicle as control. Lysates of neurons were subjected to immunoblotting using the Smurf1 antibody. I found that Smurf1 is sensitive to proteasome inhibition and accumulates upon lactacystin treatment (Figure 3.15).

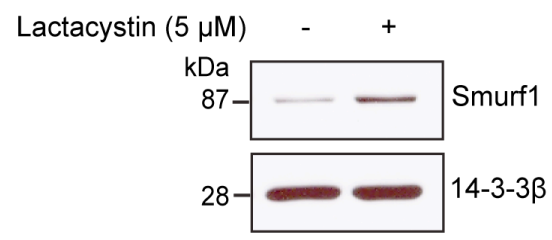

Figure 3.15 Smurf1 undergoes proteasomal degradation. Lysates of granule neurons treated with vehicle or 5 $\mu \mathrm{M}$ lactacystin for $9 \mathrm{~h}$ were subjected to immunoblotting using the Smurf1 antibody. 14-3-3 $\beta$ served as the loading control.

Further, I explored whether Smurf1 expression responds to reduced Cdh1-APC activity, as would be expected for a bona fide Cdh1 target. Smurfl protein expression was analyzed in brain lysates from $\mathrm{Cdh}^{+/+}$and $\mathrm{Cdh}^{+/-}$mice at different ages. Smurfl protein levels are induced in the Cdh1 heterozygotes (Figure 3.16A). To exclude enhanced Smurfl gene transcription in the $\mathrm{Cdh}^{+/-}$mice as the cause for increased protein levels, I performed quantitative RT-PCR to test Smurf1 mRNA levels in brain tissue from the respective ages. However, I did not detect any significant differences in Smurf1 mRNA levels between the two groups (Figure 3.16B). Collectively, my data are indicative of Cdh1-dependent posttranslational regulation of Smurfl. 

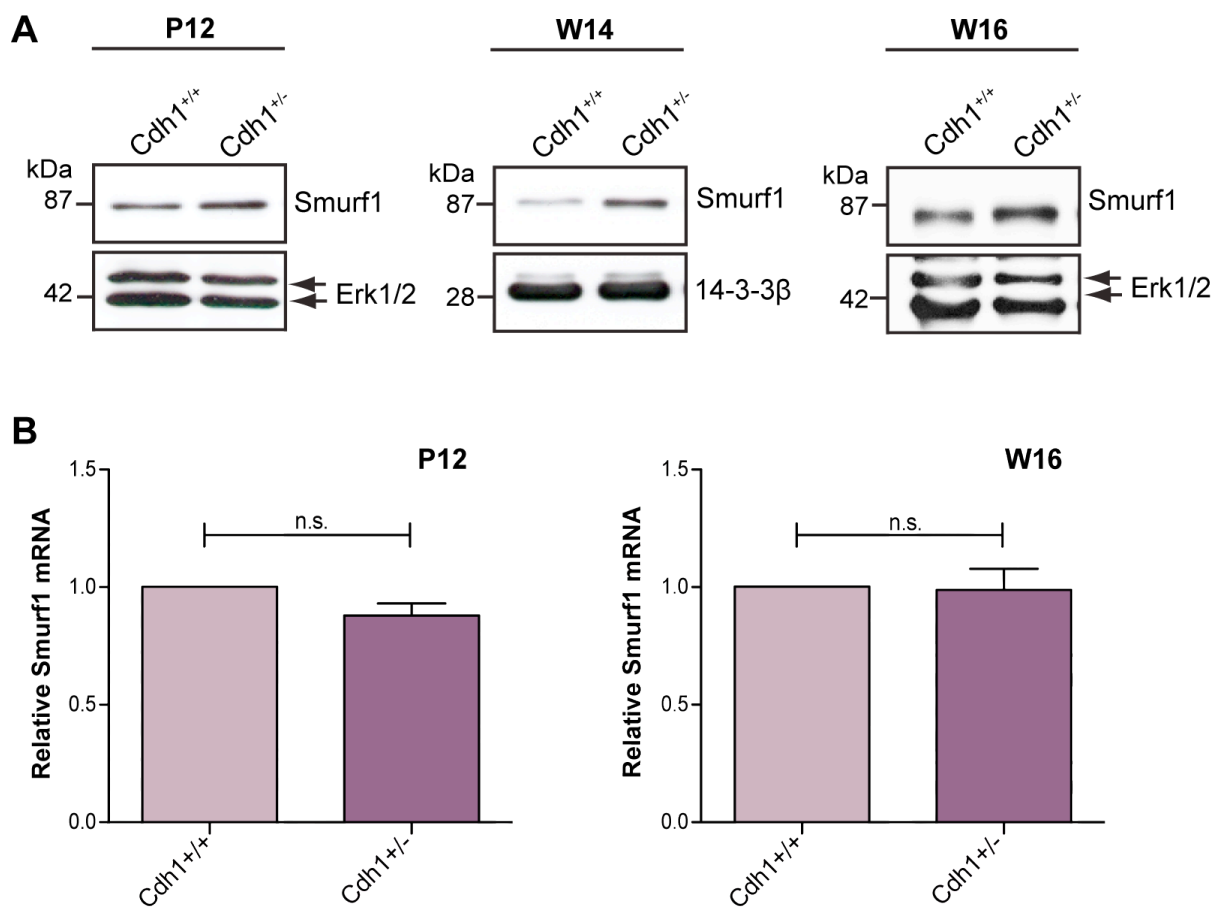

Figure 3.16 Smurf1 protein is induced in Cdh1 ${ }^{+/-}$mice. A. Cerebellar lysates of P12, W14 (week 14) and W16 (week 16) $\mathrm{Cdh}^{+/+}$and $\mathrm{Cdh}^{+/-}$mice were immunoblotted using the Smurf1 antibody. 14-3-3 $\beta$ and Erk1/2 served as loading control. B. Total mRNA was isolated from cerebella of P12 and W16 $\mathrm{Cdh}^{+/+}$and Cdh1 $1^{+/-}$mice and subjected to quantitative RT-PCR using Smurf1-specific primers. Smurf1 expression was normalized to GAPDH (P12) and $\beta$-actin (W16) (t-test, $\mathrm{p}>0.05, \mathrm{n}=8, \mathrm{P} 12$ and $\mathrm{n}=4, \mathrm{~W} 16)$.

\subsubsection{Smurf1 is poly-ubiquitinated and targeted for degradation by Cdh1-APC}

As a key confirmatory experiment to establish Smurf1 as a target of Cdh1-APC, I performed cell-based ubiquitination assays of Smurf1 upon overexpression of wild-type (WT) or a hyperphosphomimetic mutant (9D) of Cdh1 in $293 \mathrm{~T}$ cells. Cdh1 9D has been previously described as a loss-of-function mutant that fails to bind to the APC core (Huynh et al., 2009). As an initial experiment, I established that Smurf1 binds equally well to Cdh1 WT and Cdh1 9D (Figure 3.17A). Next, I transfected 293T cells with HA-Smurf1 expression plasmid together with control, Cdh1 WT or Cdh1 9D plasmids and immunoprecipitated the lysates with HA antibody and immunoblotted with a ubiquitin antibody. In the ubiquitination assay, I found that while Smurf1 is poly-ubiquitinated under control conditions, coexpression of Cdh1 WT stimulates its ubiquitination. This however, is not the case with Cdh1 9D (Figure 3.17B). 

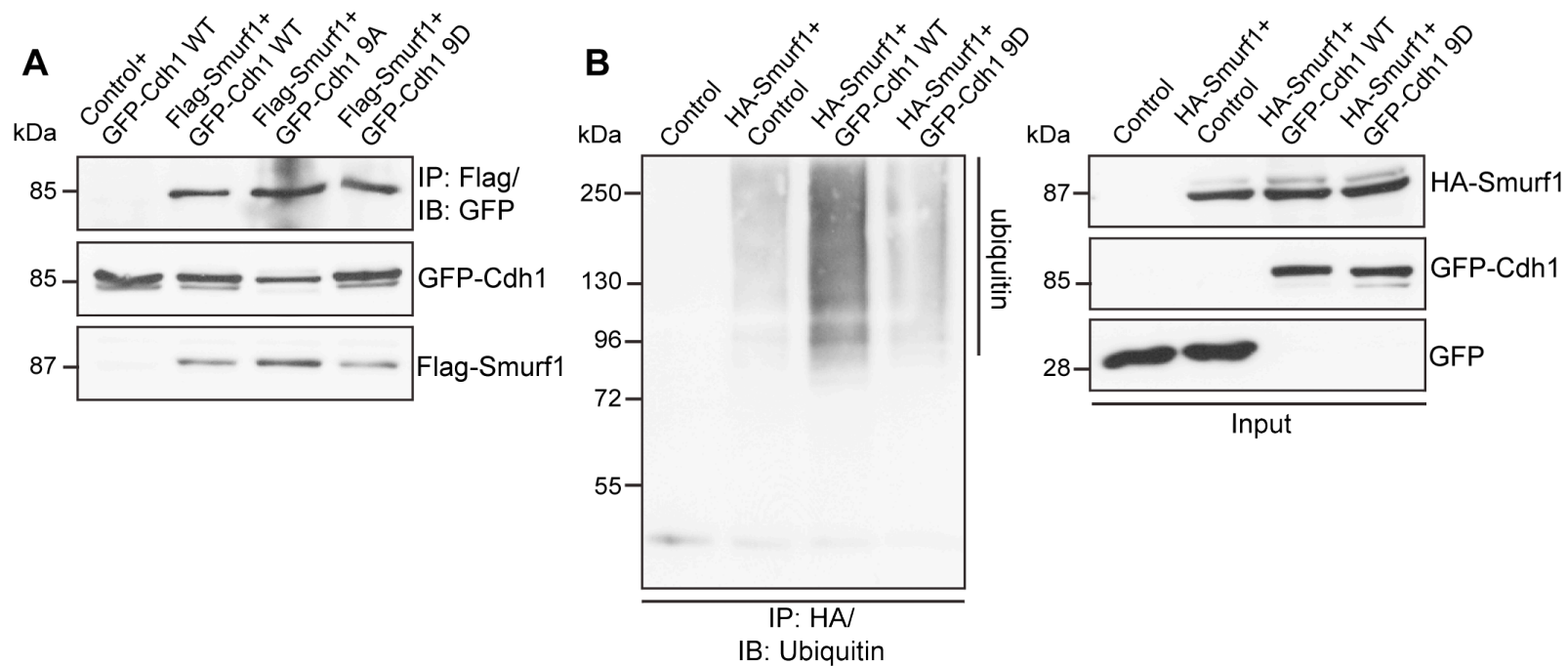

Figure 3.17 Smurf1 is poly-ubiquitinated by Cdh1-APC. A. Lysates of 293T cells transfected with control pCMV5 and GFP-Cdh1, or Flag-Smurf1 expression plasmid together with GFP-tagged Cdh1 wild type (WT), Cdh1 9A (hypophosphorylated form) or Cdh1 9D (hyperphosphorylated form) plasmid were subjected to immunoprecipitation with the Flag antibody followed by immunoblotting with the GFP antibody. B. Lysates of 293T cells transfected with control vectors pCMV5 and pEGFP, or HA-Smurf1 WT together with pEGFP, Cdh1 WT or Cdh1 9D expression plasmids, were subjected to immunoprecipitation with the HA antibody followed by immunoblotting with the ubiquitin antibody.
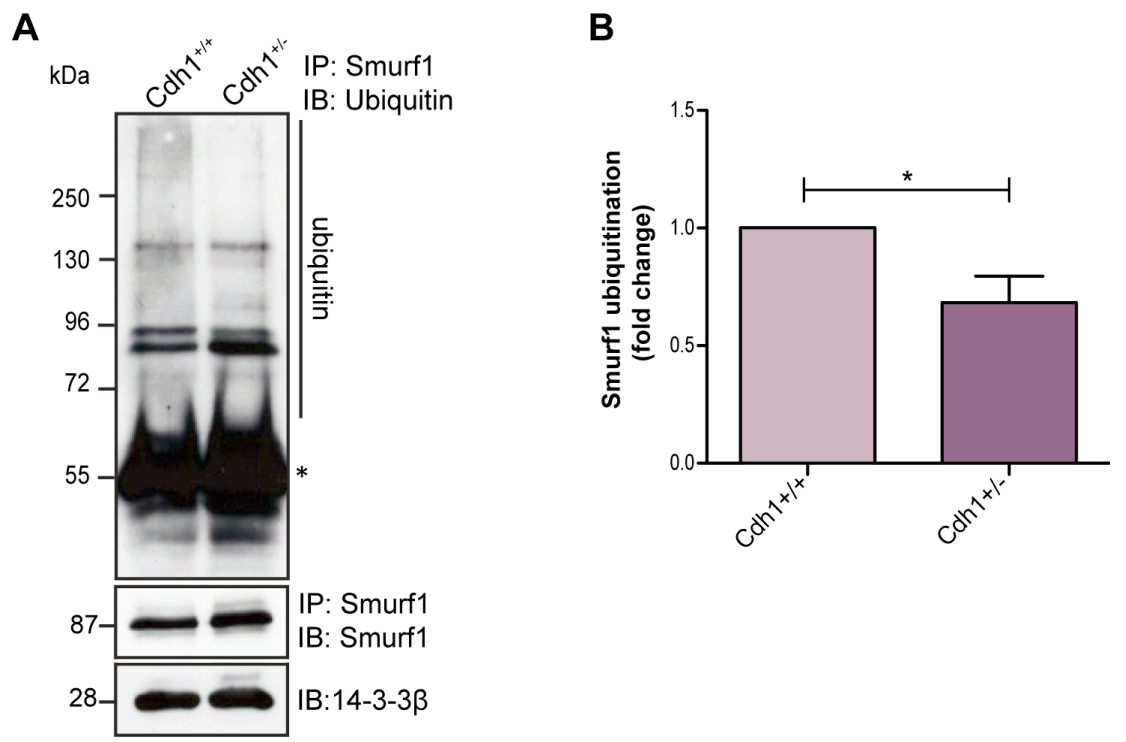

Figure 3.18 Smurf1 is poly-ubiquitinated by Cdh1-APC in vivo. A. Total brain lysates of W15 (week 15) $\mathrm{Cdh}^{+/+}$and $\mathrm{Cdh}^{+/-}$mice were subjected to immunoprecipitation with the Smurfl antibody and immunoblotting using the ubiquitin antibody. B. Intensity of Smurf1 ubiquitination was quantified and normalized to that of 14-3$3 \beta$ using ImageJ software (t-test, ${ }^{*} \mathrm{p}<0.05, \mathrm{n}=6$ pairs).

Additionally, I performed ubiquitination assays using brain lysates from adult Cdh1+/+ and Cdh1+/- mice. Lysates were immunoprecipitated using a Smurf1 antibody and immunoblotted with the ubiquitin antibody. Smurf1 ubiquitination is slightly but consistently 
reduced in Cdh1+/- brains as compared to wild-type (Figure 3.18). In essence, the results uncover the Cdh1-dependent ubiquitination of Smurf1.

\subsubsection{Two D-boxes close to its C-terminus mediate the interaction of Smurf1 with Cdh1}

In order to map the domain in Smurf1 that is crucial for its interaction with Cdh1, the human Smurfl amino acid sequence was screened for potential Cdh1-recognition motifs. These include the D-box, KEN box and A-box that were originally identified in the Cdh1 targets Cyclin B, Cdc20 and Aurora-A, respectively (Glotzer et al., 1991; Littlepage and Ruderman, 2002; Pfleger and Kirschner, 2000). Sequence analysis revealed that human Smurf1 has five potential D-boxes that share the consensus $\underline{\operatorname{RXX}} \underline{\mathrm{XXX}} \underline{\mathrm{N}}$ ) (Schematic 3A). Notably,
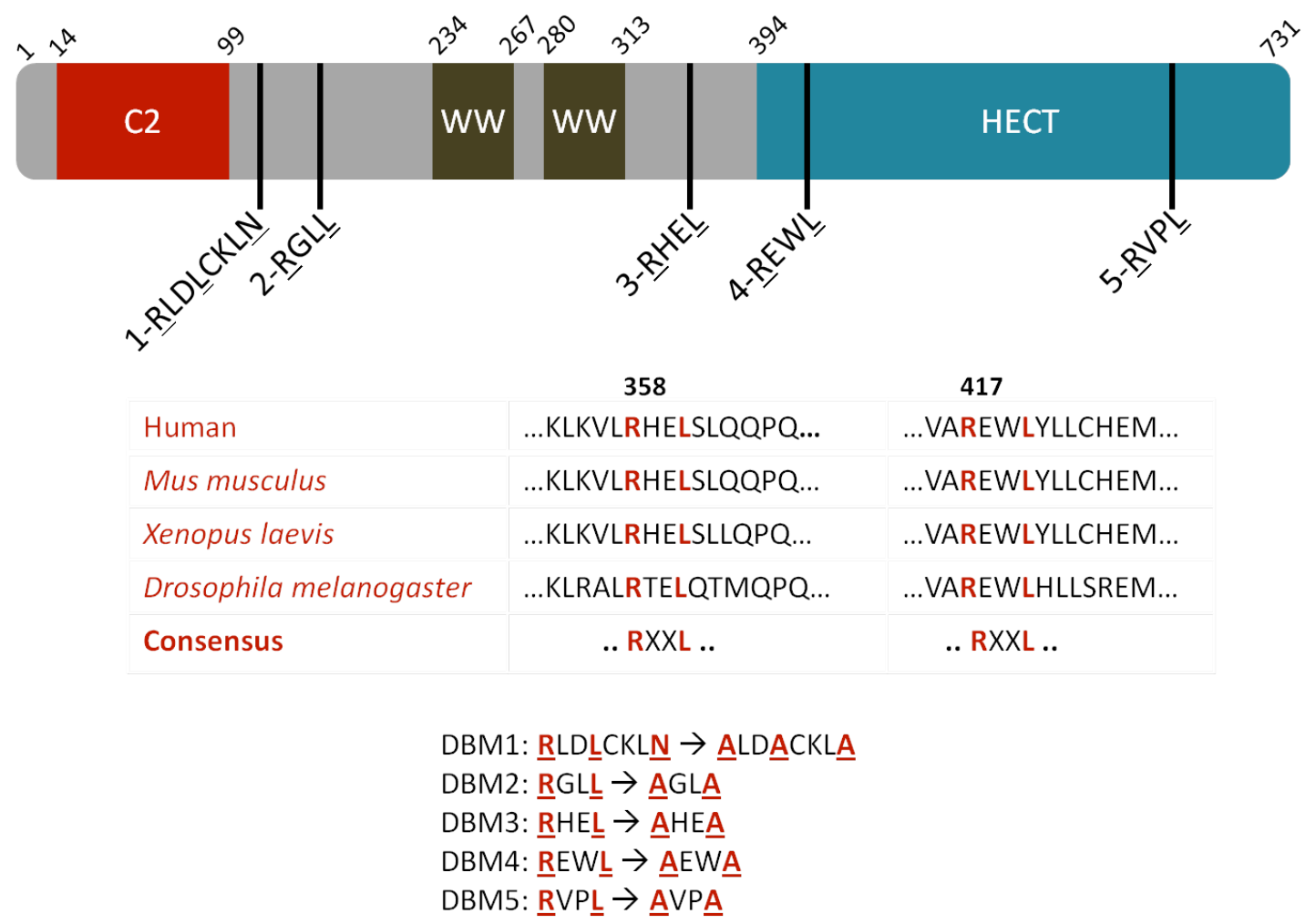

Schematic 3A. Putative D-boxes in Smurf1. Upper panel, Human Smurf1 comprises of 731 amino acids and contains an N-terminal C2 domain, two WW repeats and a C-terminal HECT domain. Smurfl harbors five putative D-boxes in its sequence. Of these, the C-terminal D-boxes 3, 4 and 5 are conserved from human to Drosophila. Lower panel, for generation of the respective D-box mutants, the conserved residues $\mathrm{R}$ and $\mathrm{L}$ were mutated to alanine.

three of the five D-boxes are conserved from human down to fly. Previous studies have reported a loss of or reduced Cdh1-binding upon mutation of the substrate D-box (Lasorella et al., 2006; Stegmuller et al., 2006; Stroschein et al., 2001). In several instances, D-box mutants exhibit a gain-of-function owing to decreased Cdh1-dependent substrate turnover (Lasorella et al., 2006; Stegmuller et al., 2006). Hence, I carried out site-directed mutagenesis to 
individually mutate all five potential D-boxes and generate the respective putative D-box mutants 1-5 (DBMs 1-5) (Schematic 3A).

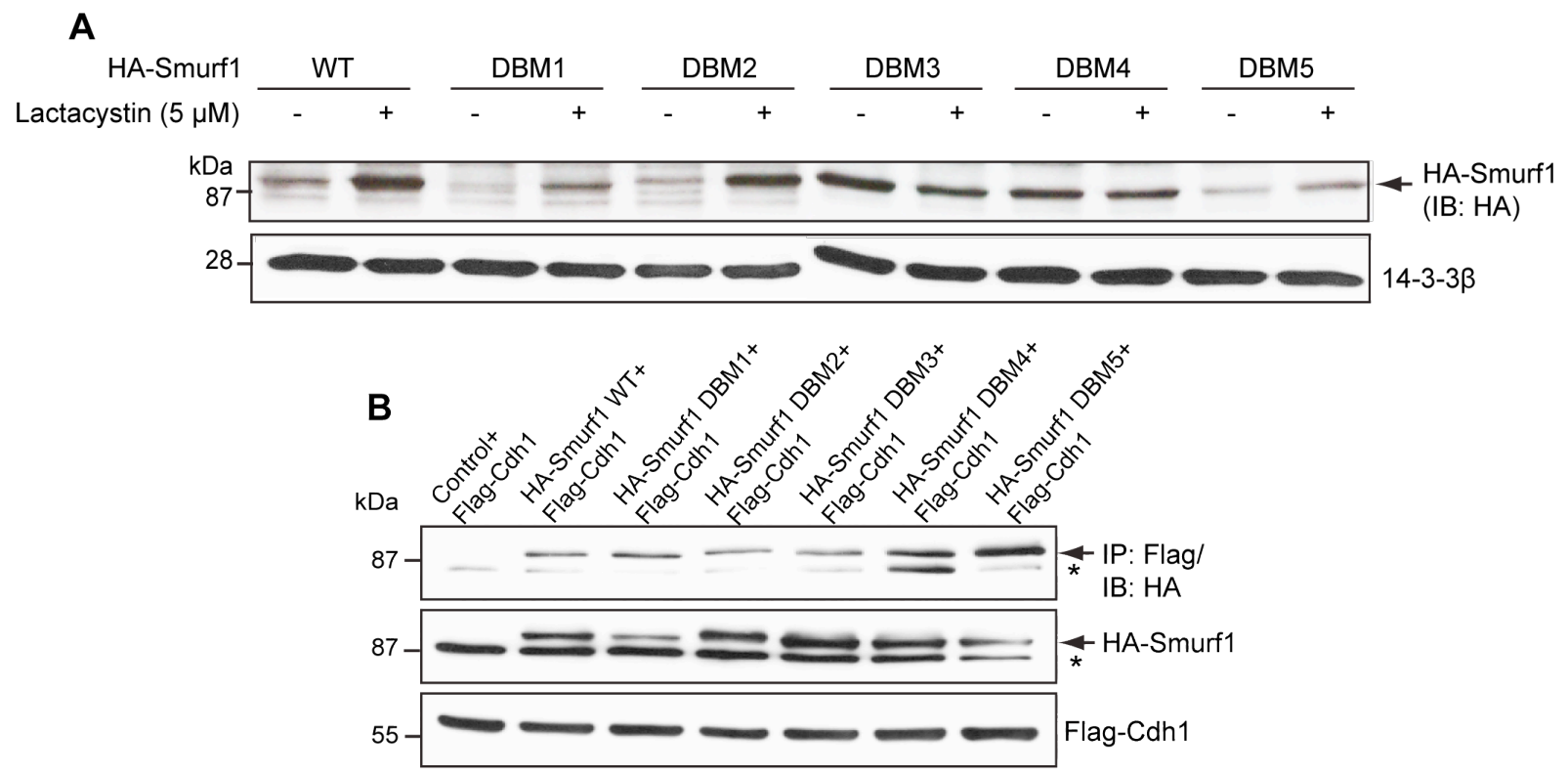

Figure 3.19 Smurf1 DBMs 3 and 4 are unresponsive to proteasomal inhibition. A. Granule neurons were transfected with HA-tagged wild-type Smurf1 or the 5 potential Smurf1 D-box mutants (DBM1-5) and treated with vehicle or $5 \mu \mathrm{M}$ lactacystin. After $9 \mathrm{~h}$, lysates were collected and immunoblotted using the HA antibody. 143-3 $\beta$ served as loading control. B. Lysates of 293T transfected with Flag-Cdh1 together with control pCMV5, HASmurf1 WT or HA-Smurf1 DBM1-5 plasmids were subjected to immunoprecipitation with the Flag antibody followed by immunoblotting with the HA antibody. Arrow and asterisk indicate HA-Smurf1 and non-specific bands, respectively.

To examine the stability of these DBMs, granule neurons were transfected at DIV 3 with plasmids encoding HA-tagged wild-type Smurf1 (Smurf1 WT) or the five potential D-box mutants Smurf1 DBM1, DBM2, DBM3, DBM4 and DBM5, and at DIV 6, subjected to lactacystin treatment. The lysates were then analysed by immunoblotting using the HA antibody. Besides wild-type Smurf1, the mutants Smurf1 DBM1, DBM2 and DBM5 accumulate upon proteasome inhibition. On the contrary, Smurf1 DBM3 and DBM4 fail to respond to lactacystin treatment but instead, show a high baseline expression for the same amount of lysate probed (Figure 3.19A). My observations suggest that mutations of the putative D-boxes 3 and 4 stabilize Smurfl possibly due to decreased Cdh1-dependent turnover.

\subsubsection{Smurf1 double D-box mutant exhibits greater stability and reduced affinity for}

Cdh1 as compared to the wild-type protein

To determine whether mutations of the putative Smurfl D-boxes 3 and 4 affect their binding affinity to Cdh1, I tested the interaction of each of the Smurf1 DBMs with Cdh1. 293T cells were transfected with a Flag-Cdh1 plasmid together with HA-tagged wild-type or Smurf1 
DBM1-5 expression plasmids. Lysates were subjected to immunoprecipitation using the Flag antibody followed by immunoblotting with HA antibody. However, I found no decrease in the strength of interaction of any of the DBMs with Cdh1 as compared to that of Smurf1 WT with Cdh1 (Figure 3.19B). Hence, I went on to generate the double D-box mutant plasmid Smurf1 DBM3/4 and expressed it together with a Cdh1 plasmid in 293T cells. Coimmunoprecipitation analysis revealed a conspicuous reduction in the binding affinity of the double mutant to Cdh1 (Figure 3.20A).
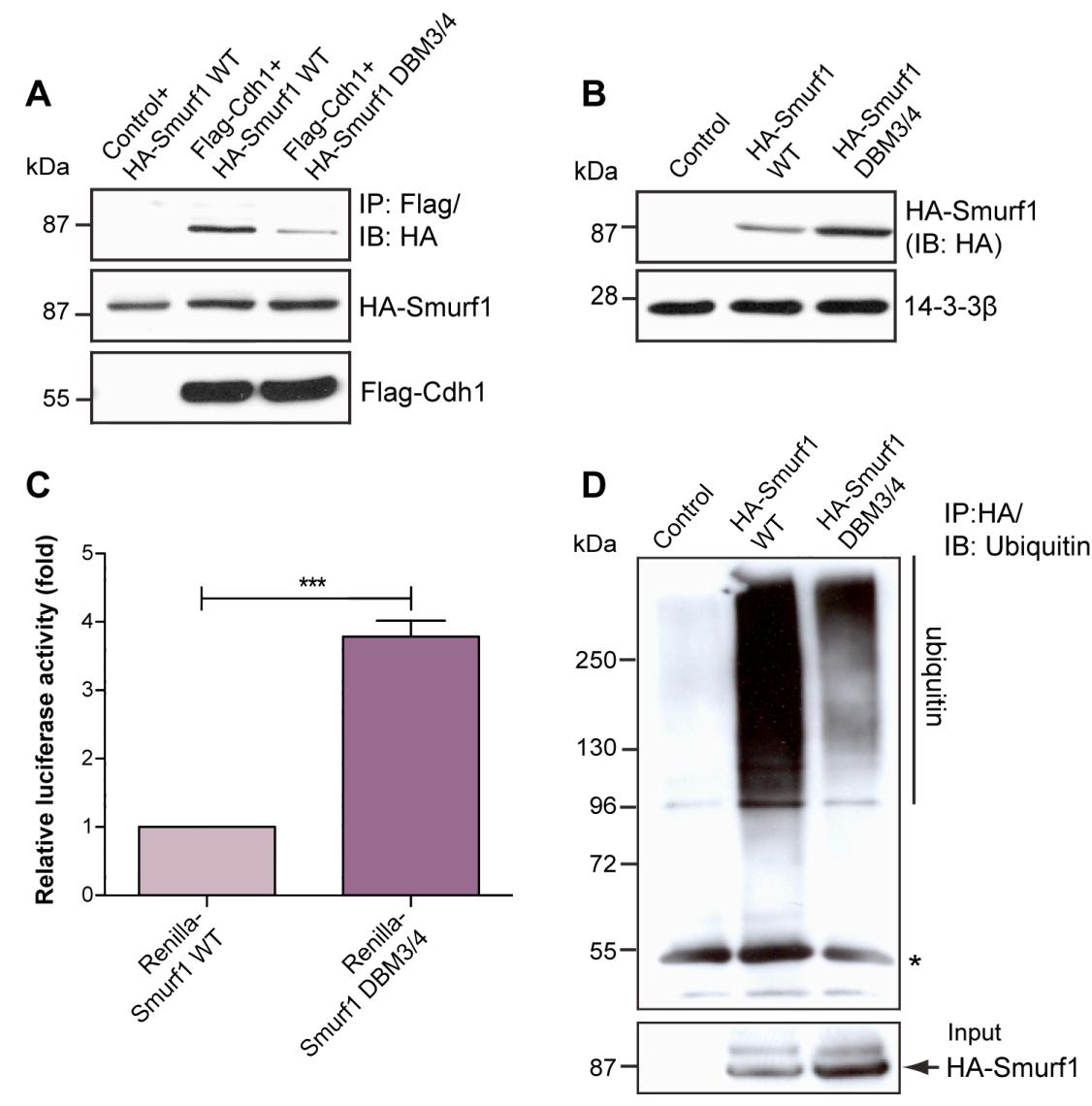

Figure 3.20 Smurf1 DBM3/4 is stabilized. A. Lysates of $293 \mathrm{~T}$ cells transfected with a plasmid encoding HASmurf1 WT and empty vector pCMV5 or the Flag-Cdh1 plasmid, or HA-Smurf1 DBM3/4 and Flag-Cdh1 plasmids were subjected to immunoprecipitation using the Flag antibody followed by immunoblotting with the HA antibody. B. Lysates of 293 T transfected with equal amounts of control pCMV5, HA-Smurf1 WT or HASmurf1 DBM3/4 plasmid were subjected to immunoblotting with the HA antibody. 14-3-3 $\beta$ served as loading control. C. 293T cells transfected with Renilla-Smurf1 WT or Renilla-Smurf1 DBM3/4 expression plasmid together with the SV40 firefly luciferase (pGL3 promoter) plasmid, the latter to serve as internal control for transfection efficiency, were subjected to luciferase assay (t-test, $\mathrm{p}<0.0001, \mathrm{n}=8$ ). Luciferase assays were performed by Dr. Judith Stegmüller, MPI-EM, Göttingen. D. Lysates of 293T transfected with control vector pCMV5, HA-Smurf1 WT or HA-Smurf1 DBM3/4 plasmids were subjected to immunoprecipitation using the HA antibody followed by immunoblotting using the ubiquitin antibody. Asterisk indicates $\operatorname{IgG}_{\mathrm{H}}$.

To corroborate the increase in Smurfl stability as the result of the double D-box mutations, Smurf1 DBM3/4 expression was tested first by immunoblotting using 293T cell lysates and in luciferase assays. 293T cells transfected with equal amounts of HA-tagged 
Smurf1 WT and Smurf1 DBM3/4 plasmids were lysed and probed for protein expression using the HA antibody. HA-Smurf1 DBM3/4 is dramatically stabilized as compared to Smurf1 WT (Figure 3.20B). Granule neurons transfected with Renilla-Smurf1 WT or Renilla-Smurf1 DBM3/4 together with internal control SV40 firefly luciferase plasmid were subjected to dual luciferase assay. Luciferase assay was performed by Dr. Judith Stegmüller, MPI-EM, Göttingen. Renilla-Smurf1 DBM3/4 exhibits a three-fold increase in activity as compared to Renilla-Smurf1 WT (Figure 3.20C).

Additionally, I transfected 293T cells with control, HA-tagged Smurf1 WT or Smurf1 DBM3/4 plasmids and subjected the lysates to immunoprecipitation using the HA antibody followed by immunoblotting with the ubiquitin antibody. The results of the cell-based ubiquitination assay revealed that Smurf1 DBM3/4 is ubiquitinated to a much lesser extent than the WT (Figure 3.20D). To sum up, the data imply Smurf1 D-boxes 3 and 4 as recognition motifs in Cdh1-dependent modulation of Smurf1 stability.

\subsubsection{Smurf1 enhances axon growth independently of the neuronal cell type}
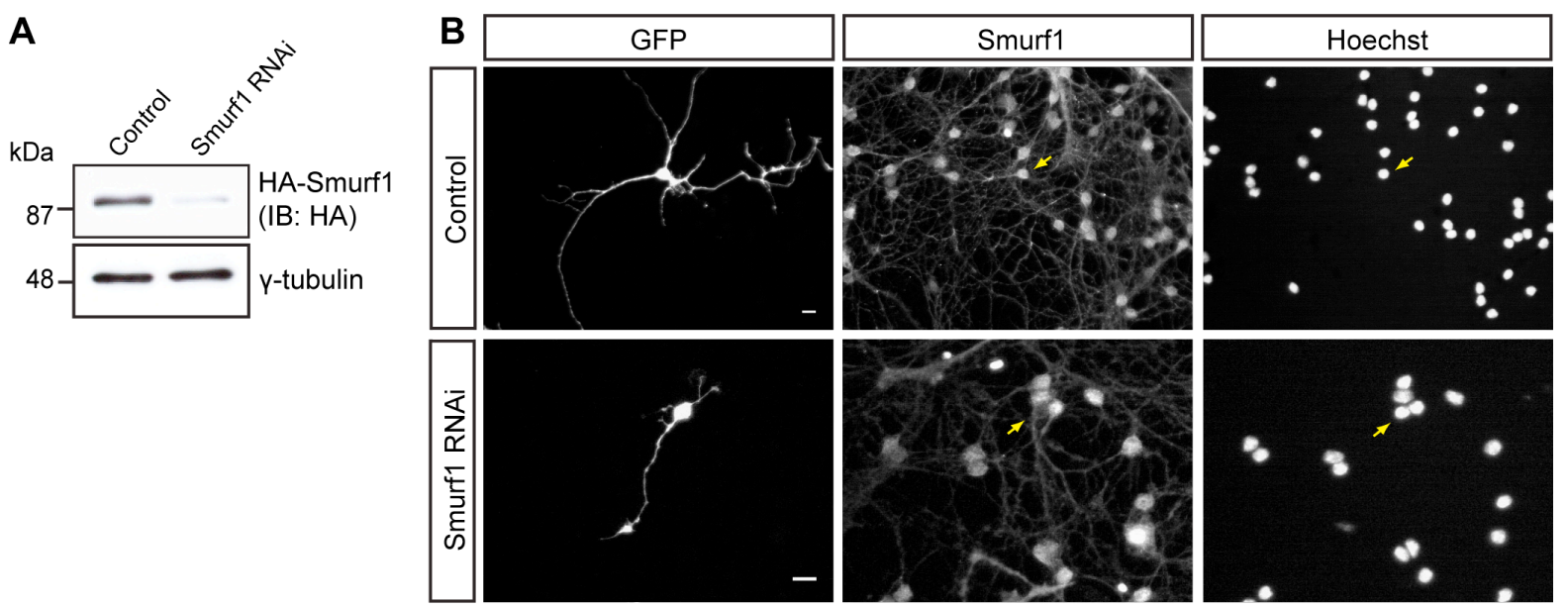

Figure 3.21 Validation of Smurf1 RNAi plasmid. A. 293T cells were transfected with a plasmid encoding HASmurf1 together with control vector U6 or the Smurf1 RNAi plasmid. Lysates were subjected to immunoblotting using the HA antibody. $\gamma$-tubulin served as the loading control. B. Granule neurons were transfected with control vector U6 or the Smurf1 RNAi plasmid together with a plasmid encoding farnesylated GFP and subjected to immunocytochemistry with the Smurf1 and the GFP antibodies. Arrows in representative images indicate control transfected Smurf1-positive neurons and Smurf1 knockdown neurons. Scale bar equals $10 \mu \mathrm{m}$.

Recent work ascribes a role for Smurfl in the regulation of neurite outgrowth and axon initiation (Cheng et al., 2011; Sato and Heuckeroth, 2008). My results propose a Cdh1dependent protein turnover of Smurf1 and provoke questions concerning the involvement of Smurf1 in axon growth regulation in the Cdh1-APC/RhoA cascade. To resolve the function of 
Smurf1 in axon growth, a Smurf1 RNAi plasmid was generated based on a previously described target region (Boyer et al., 2006) and Smurfl knockdown was validated in heterologous cells. 293T cells were transfected with Smurf1 WT together with control or Smurf1 RNAi plasmid. The lysates were analyzed for Smurf1 levels. Smurfl RNAi induces a dramatic knockdown of Smurf1 WT (Figure 3.21A). The RNAi plasmid was further tested for robust knockdown of endogenous Smurf1 in granule neurons (Figure 3.21B). About $76 \%$ of GFP-positive neurons are Smurf1-negative in the knockdown condition as compared to none in the control.
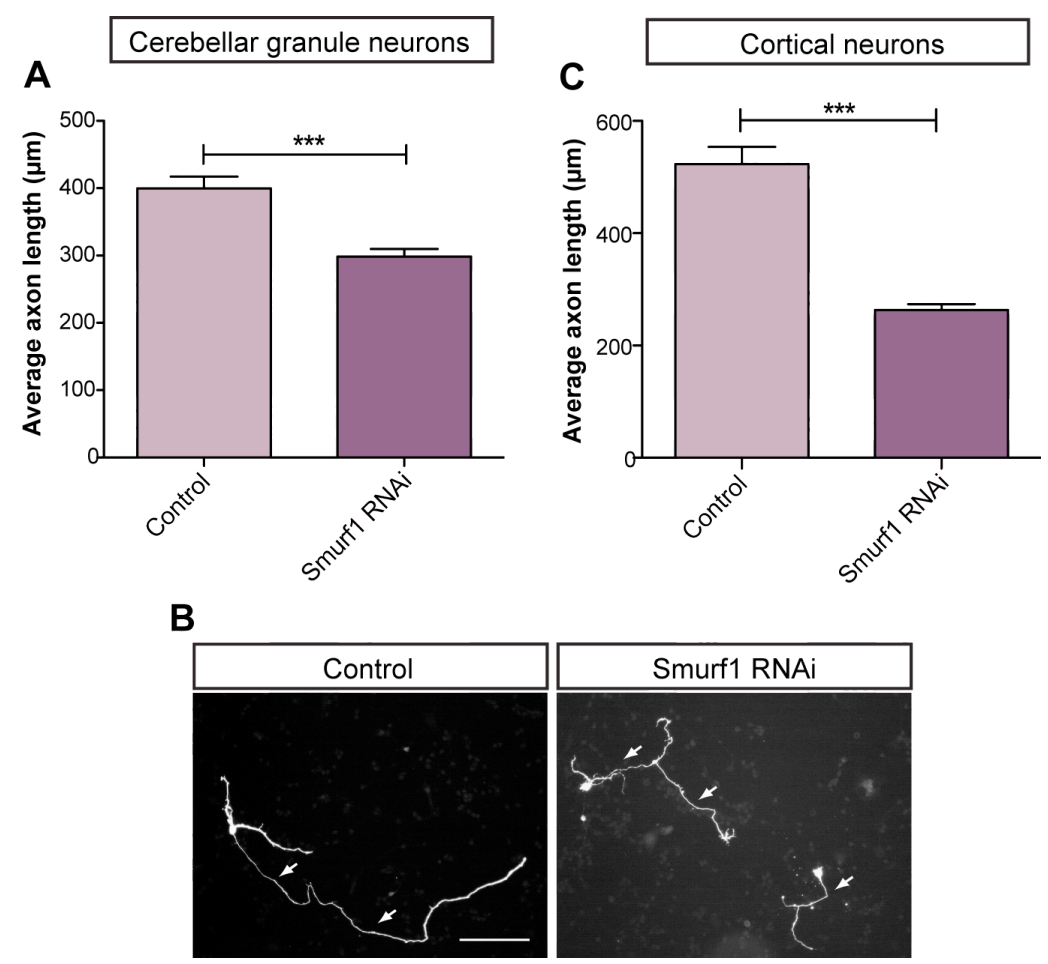

Figure 3.22 Smurf1 promotes axon growth. A. Cerebellar granule neurons were transfected at DIV 0 with control vector U6 or the Smurf1 RNAi plasmid together with the GFP and BCL $\mathrm{XL}_{\mathrm{XL}}$ plasmids and maintained in conditioned medium. At DIV 4, neurons were analyzed as described in Figure 3.3. A total of 384 neurons was measured (t-test, $* * * \mathrm{p}<0.0001$, values indicate mean + SEM). B. Representative images of transfected neurons in A. Scale bar equals $100 \mu \mathrm{m}$. Arrows indicate axons. C. Cortical neurons were transfected at DIV 1 with the control vector U6 or the Smurf1 RNAi plasmid. At DIV 4, neurons were analyzed as in A. A total of 292 neurons was measured (t-test, $* * * \mathrm{p}<0.0001)$. Cortical neuron axon assays were performed by Shih-Ju Lee, Stegmüller Lab, MPI-EM, Göttingen.

Next, cultured granule neurons were transfected at DIV 0 with either control or the Smurf1 RNAi plasmid and subjected to axon growth assays at DIV 4. Axon length measurements revealed that Smurf1 knockdown neurons possess significantly shorter axons than control neurons, an effect that recurs also in cortical neurons (Figure 3.22). Cortical neuron axon assays were performed by Shih-Ju Lee, Stegmüller Lab, MPI-EM, Göttingen. These experiments suggest an axon growth-promoting function for Smurfl independent of 
neuronal cell type. To attribute the short-axon phenotype specifically to Smurf1 knockdown and to rule out off-target effects of the hairpin, a Smurf1 rescue plasmid (Smurf1-Res) was generated in a way that the full length Smurfl contains four silent mutations in the RNAi target region. The rescue construct was subsequently tested in $293 \mathrm{~T}$ cells for its ability to evade the knockdown effect (Figure 3.23A). Next, in rescue assays, granule neurons were transfected with empty vectors only, or the Smurf1 RNAi plasmid together with empty vector or Smurf1 rescue plasmid. Expression of Smurf1-Res efficiently restores axonal length to basal levels ensuring the specificity of the Smurf1 RNAi phenotype (Figure 3.23B, C).
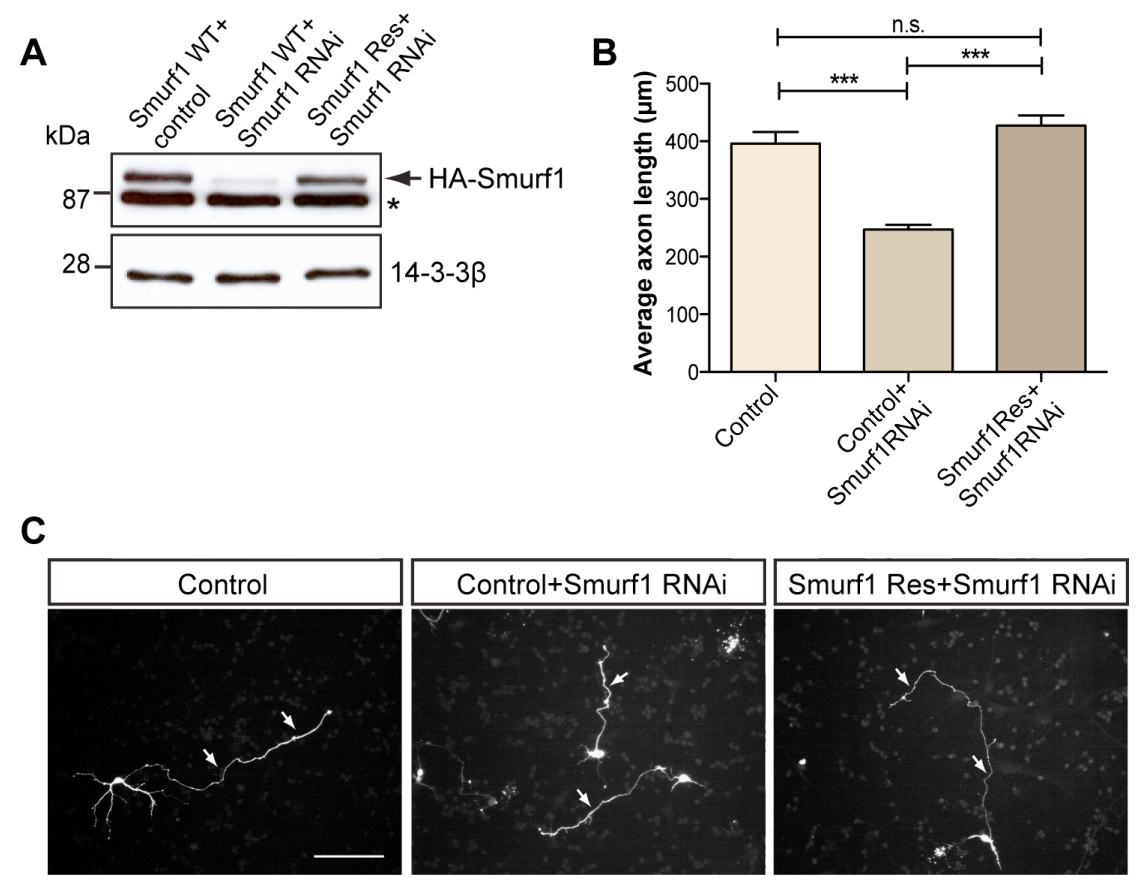

Figure 3.23 Smurf1 rescue plasmid reverts the Smurf1 knockdown phenotype. A. Lysates of 293T cells transfected with HA-tagged Smurf1 WT plasmid and empty vector U6, or Smurf1 RNAi plasmid and HA-Smurf1 WT or HA-Smurf1 rescue (Smurf1-Res) plasmid were immunoblotted using the HA antibody. 14-3-3 $\beta$ served as loading control. Asterisk indicates non-specific band. B. Cerebellar granule neurons were transfected at DIV 0 with control vector pCMV5 and U6, or Smurf1 RNAi plasmid together with empty vector pCMV5 or Smurf1-Res plasmid and maintained in conditioned medium. At DIV 4, neurons were analyzed as in Figure 3.3. A total of 425 neurons was measured (ANOVA, $* * * p<0.0001$, values indicate mean + SEM). C. Representative images of transfected neurons in B. Scale bar equals $100 \mu \mathrm{m}$. Arrows indicate axons.

Further, to test whether the axon growth-promoting effect of Smurf1 is dependent on its nuclear and/or cytosolic localization, NES-Smurf1 and NLS-Smurf1 rescue plasmids (HANES-Smurf1-Res and HA-NLS-Smurf1-Res, respectively) were generated by appending a nuclear exclusion signal (NES) or nuclear localization signal (NLS) to the N-terminus of the Smurf1 rescue sequence. The NES-Smurf1 and NLS-Smurf1 rescue plasmids were first transfected in $293 \mathrm{~T}$ cells and granule neurons and tested for the intended localization by immunocytochemistry using the HA antibody (Fig 3.24A, B). These plasmids were 

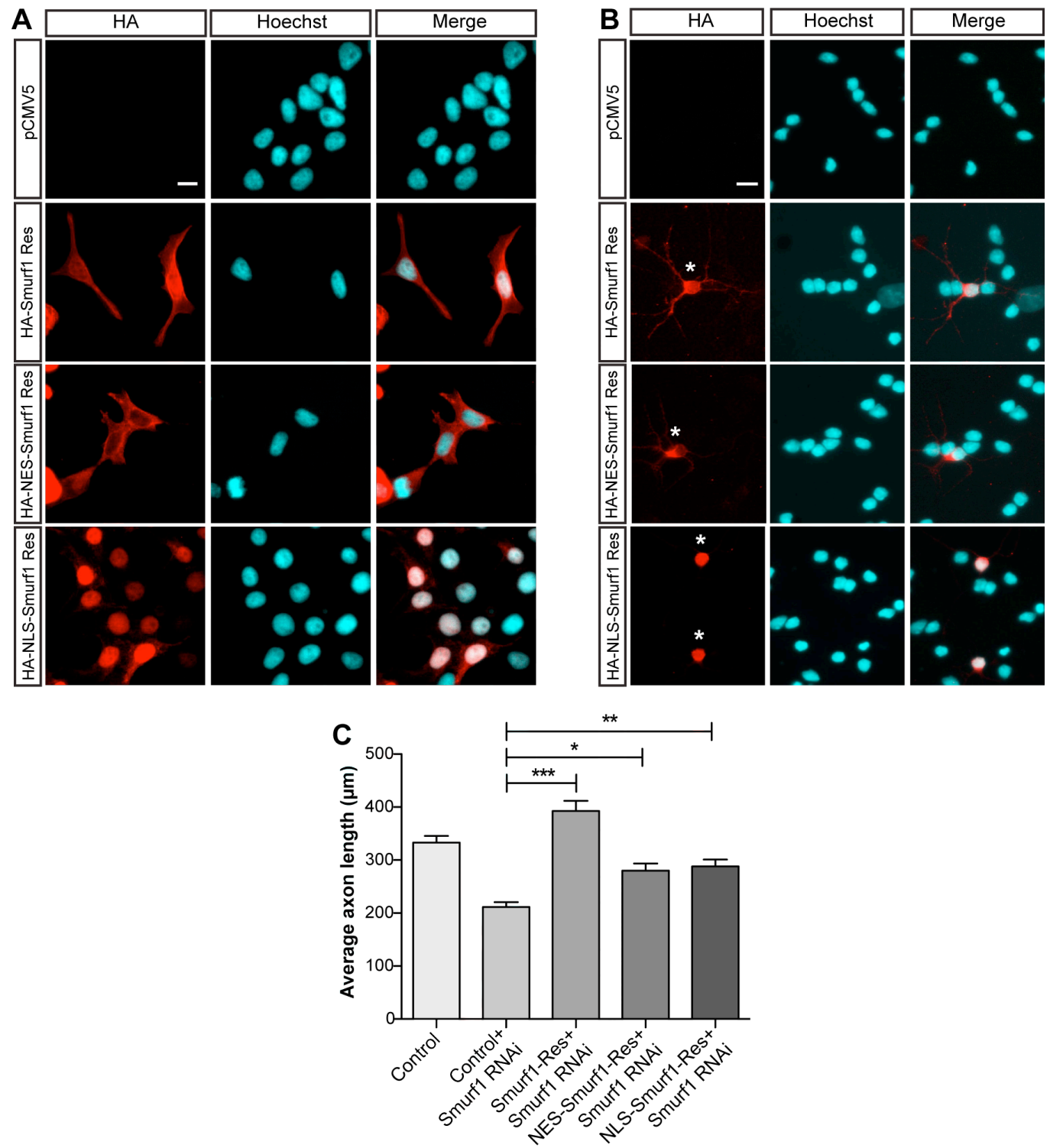

Figure 3.24 Nuclear and cytoplasmic localization of Smurf1 are essential for axon growth. A. 293T cells transfected with control vector pCMV5, HA-Smurf1-Res, HA-NES-Smurf1-Res or HA-NLS-Smurf1-Res plasmid were subjected to immunocytochemistry using the HA antibody and Hoechst 33258. Scale bar equals $10 \mu \mathrm{m}$. B. Granule neurons transfected at DIV 1 with control vector pCMV5, HA-Smurf1-Res, HA-NES-Smurf1-Res or HA-NLS-Smurf1-Res plasmid were fixed at DIV 3 and subjected to immunostaining using the HA antibody and Hoechst 33258. Asterisks indicate soma. Scale bar equals $10 \mu \mathrm{m}$. C. Granule neurons transfected with control vectors U6 and pCMV5, Smurf1 RNAi and pCMV5 plasmid, or Smurf1 RNAi plasmid together with Smurf1-Res, NES-Smurf1-Res or NLS-Smurf1-Res expression plasmid, were maintained in conditioned medium and analyzed at DIV 4 as described in Figure 3.3. A total of 1076 neurons were measured (ANOVA, ${ }^{* * *} p<0.0001,{ }^{* *} p<0.001$, $* \mathrm{p}<0.05)$.

successively used in rescue assays. At DIV 0, granule neurons were transfected with empty vectors only, empty vector and Smurf1 RNAi plasmid, or Smurf1 RNAi plasmid together with either Smurf1-Res, NES-Smurf1-Res or NLS-Smurf1-Res and axon assays were performed after subjecting the neurons to immunocytochemistry at DIV 4. While Smurf1-Res completely restores the axon length of the Smurf1 knockdown neurons to control conditions, NESSmurf1-Res or NLS-Smurf1-Res produces only a partial rescue effect (Figure 3.24C). Thus, 
both nuclear and cytoplasmic localization of Smurf1 are crucial to its role in axon growth regulation.

\subsubsection{Smurf1 acts downstream of $\mathrm{Cdh} 1$ in a linear pathway in the control of axon growth}

As an axon growth-promoting protein whose stability is controlled by Cdh1-APC, I postulated that Smurfl operates in a linear pathway downstream of Cdh1-APC in neuronal function. To test this hypothesis, I carried out epistasis analysis in which granule neurons transfected with control, Cdh1 RNAi or Smurf1 RNAi plasmid alone or both Cdh1 and Smurf1 RNAi plasmids were subjected to axon growth assays. While Cdh1 knockdown enhances and Smurf1 knockdown inhibits axon growth, knockdown of both Cdh1 and Smurf1 results in a significant decrease in axon length (Figure 3.25). The double knockdown phenotype resembles that of Smurf1 knockdown alone suggesting that the Smurf1 RNAi phenotype overrules the induction of axon growth mediated by Cdh1 knockdown. In other words, my data indicate that Smurf1 operates downstream of Cdh1 in axon growth regulation.
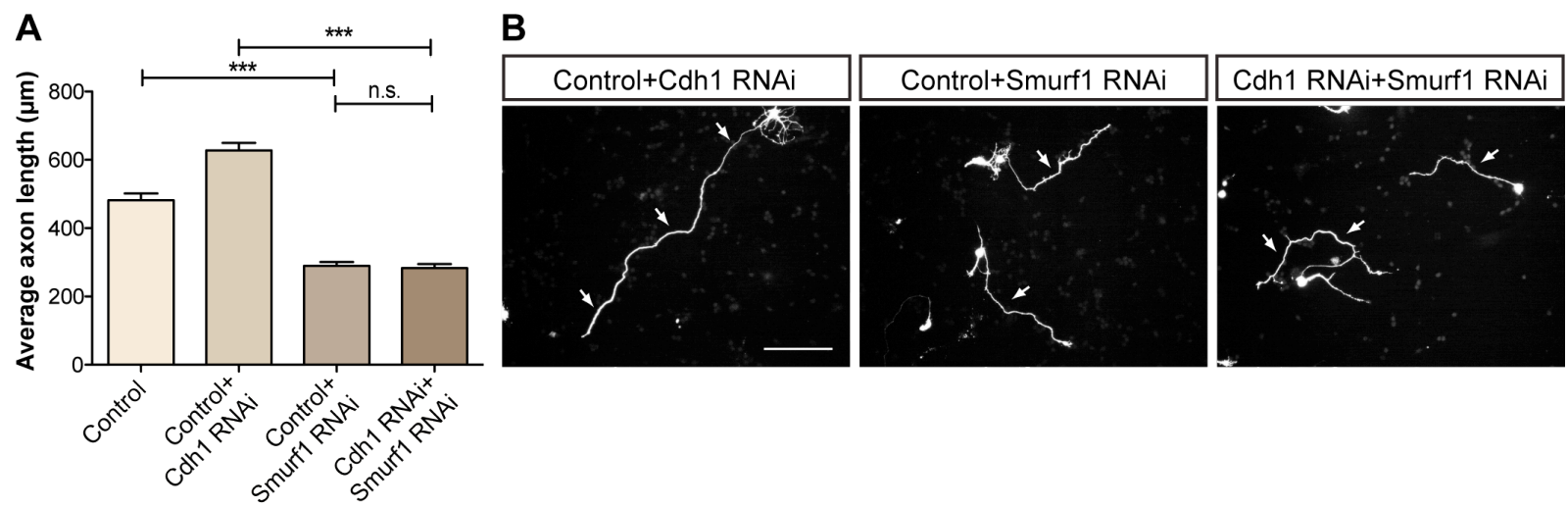

Figure 3.25 Smurf1 promotes axon growth downstream of Cdh1-APC. A. Neurons transfected with control U6, U6 and Cdh1 RNAi, or U6 and Smurf1 RNAi or both Cdh1 RNAi and Smurf1 RNAi plasmids were maintained in conditioned medium and analyzed at DIV 4 as described in Figure 3.3. A total of 666 neurons was measured (ANOVA, $* * * p<0.0001$, values indicate mean + SEM). B. Representative images of transfected neurons in A. Scale bar equals $100 \mu \mathrm{m}$. Arrows indicate axons.

The transcription factors, SnoN and Id2 have been previously described as targets of Cdh1-APC in the control of axon growth (Lasorella et al., 2006; Stegmuller et al., 2006). Thus, I asked whether the Cdh1-APC/Smurf1 pathway involves either or both of the two known Cdh1 targets. To test if Smurf1 operates in a linear pathway together with SnoN and/or Id2, I performed the following epistasis experiments. Granule neurons were transfected with control plasmids, Smurf1 RNAi plasmid or SnoN (or Id2) DBM plasmid or both of Smurf1 RNAi and SnoN (or Id2) DBM expression plasmids. Both SnoN DBM and Id2 DBM exhibit a strong 
axon growth-promoting effect as a result of enhanced stability (Lasorella et al., 2006; Stegmuller et al., 2006). Axon assays at DIV 4 showed that Smurfl knockdown markedly reduces and SnoN DBM or Id2 DBM significantly promotes axon growth as compared to control conditions. However, expression of both Smurf1 RNAi and SnoN DBM (or Id2 DBM) results in axons that resemble neither phenotype. The average axon length in this condition is not only significantly longer than that of the Smurf1 RNAi but also significantly shorter than that of the SnoN DBM (or Id2 DBM) neurons suggesting that neither phenotype is epistatic to the other (Figures 3.26). Collectively, Smurf1 operates downstream of Cdh1-APC in the control of axon growth but in a pathway independent of SnoN or Id2.

A

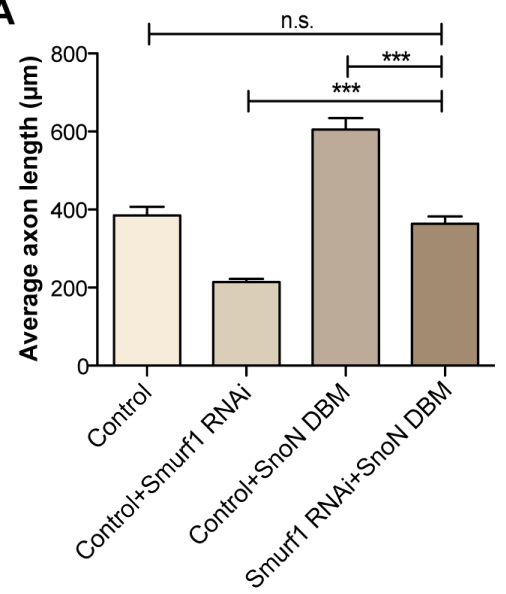

B

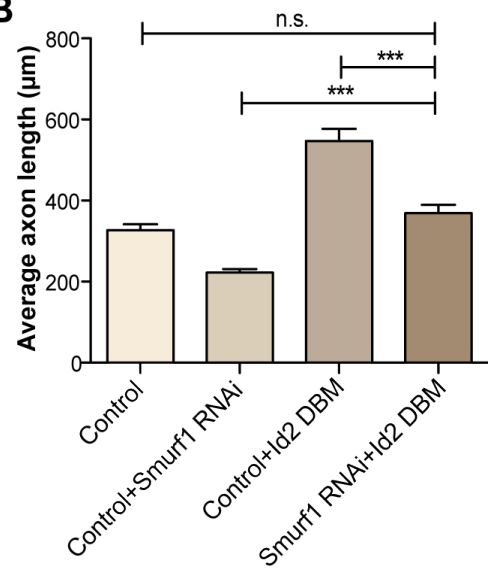

Figure 3.26 Smurf1 controls axon growth independent of SnoN or Id2. A. Neurons transfected with control pCMV5 and U6 or Smurf1 RNAi, U6 and SnoN DBM, or both SnoN DBM and Smurf1 RNAi plasmids were maintained in conditioned medium and analyzed at DIV 4 as described in Figure 3.3. A total of 510 neurons was measured (ANOVA, $* * * \mathrm{p}<0.0001$, n.s. $=$ non-significant, values indicate mean $+\mathrm{SEM}$ ). B. Neurons transfected with control pCDNA3 and U6 or Smurf1 RNAi, U6 and Id2 DBM, or both Id2 DBM and Smurf1 RNAi plasmids were maintained in conditioned medium and analyzed at DIV 4 as described in Figure 3.3. A total of 493 neurons were measured (ANOVA, $* * * p<0.0001$, n.s. $=$ non-significant, values indicate mean + SEM).

\subsubsection{The double D-box mutant of Smurf1 exhibits a gain-of-function in the regulation of axon growth}

Given that the Smurf1 double mutant Smurf1 DBM3/4 binds Cdh1 only weakly and exhibits a greater stability, I determined a potential gain-of-function effect of Smurf1 DBM3/4 in axon growth assays. Granule neurons were transfected at DIV 0 with control vector, Smurf1 WT or Smurf1 DBM3/4 plasmid and subjected to axon growth measurements at DIV 3. The results of the analyses revealed that Smurf1 DBM3/4 outperforms Smurf1 WT in stimulating axon growth. While Smurfl WT produces a barely significant increase in axon length compared to control neurons, Smurf1 DBM3/4 overexpression results in approximately two- 
fold longer axons than the control condition (Figure 3.27). In summary, Smurf1 DBM3/4 as a result of its enhanced stability potently stimulates axonal growth.

A

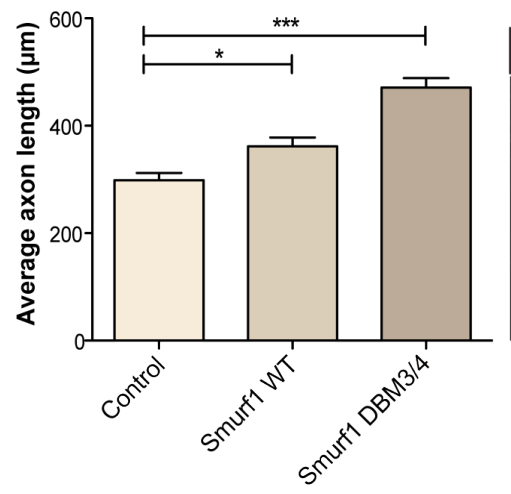

B

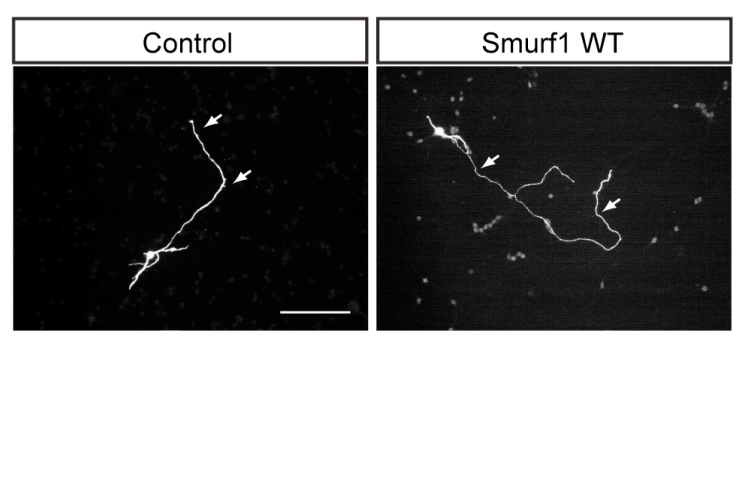

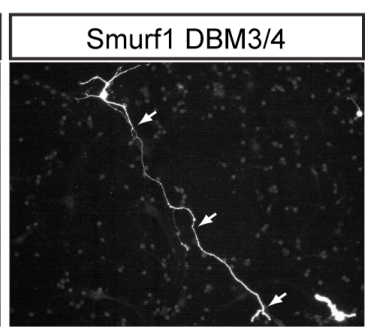

Figure 3.27 Smurf1 DBM3/4 exhibits a gain-of-function in axon growth. A. Neurons were transfected with control vector pCMV5, Smurf1 WT or Smurf1 DBM3/4 expression plasmid, maintained in conditioned medium and analyzed at DIV 3 as in Figure 3.3. A total of 617 neurons was measured (ANOVA, ${ }^{* * *} p<0.0001,{ }^{*} p<0.05$ values indicate mean + SEM). B. Representative images of transfected neurons in A. Scale bar equals $100 \mu \mathrm{m}$. Arrows indicate axons.

\subsubsection{Smurf1 promotes axon growth downstream of Cdh1-APC by degrading RhoA}

Recalling the foremost question of whether Smurfl is the relevant component of the Cdh1-APC/RhoA pathway of axon growth control as the regulator of RhoA, I next sought to address the significance of RhoA degradation by Smurfl in axon growth regulation downstream of Cdh1-APC. The amino acid sequence of RhoA harbors two evolutionarily conserved lysine residues, K6 and K7, key to Smurf1-dependent ubiquitination of the Rho GTPase. Mutation of the critical lysines has been shown to render RhoA refractory to degradation by Smurf1 (Ozdamar et al., 2005). Thus, a Smurf1-degradation resistant form of RhoA, RhoA K6,7R was generated by site-directed mutagenesis and used in axon assays together with Smurf1 DBM3/4.

Granule neurons were transfected with control vectors or Smurf1 DBM3/4 plasmid together with control vector, RhoA WT or RhoA K6,7R plasmids. Analyses revealed that RhoA WT significantly reduces axonal length in the Smurf1 DBM3/4 neurons and that the Smurf1-degradation resistant variant RhoA K6,7R abates axon growth in these neurons in an even more pronounced way (Figure 3.28A). Furthermore, this effect of RhoA is specific since overexpression of another Rho GTPase Cdc42 does not have any effect on Smurf1 DBM3/4induced axon growth (Figure 3.28B). In essence, Smurf1 exerts its axon growth promoting effect by regulating RhoA. To further place this finding in the Cdh1-APC/RhoA axis, a similar axon growth assay was performed, this time transfecting granule neurons with control vector, RhoA WT or RhoA K6,7R together with Cdh1 RNAi plasmid. Again, while RhoA WT 
significantly restores axon lengths in Cdh1 knockdown neurons to control conditions (as described in Section 3.2.1), RhoA K6,7R produces an even more dramatic inhibition of axon growth in these neurons (Figure 3.28C). Taken together, Smurfl degradation of RhoA is a crucial downstream event in the control of axon growth by Cdh1-APC.

A

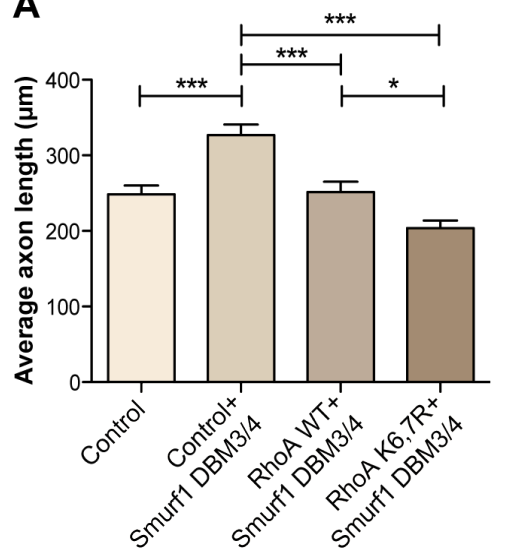

B

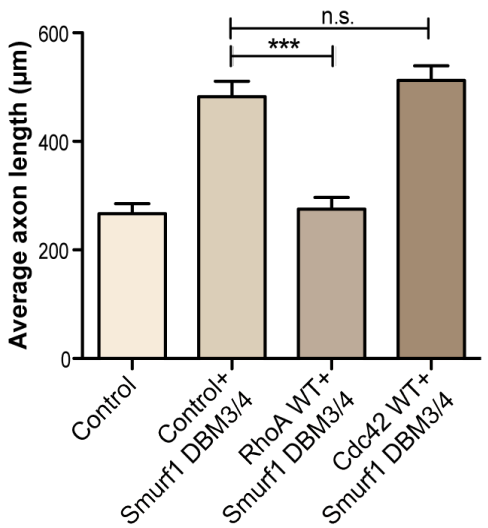

C

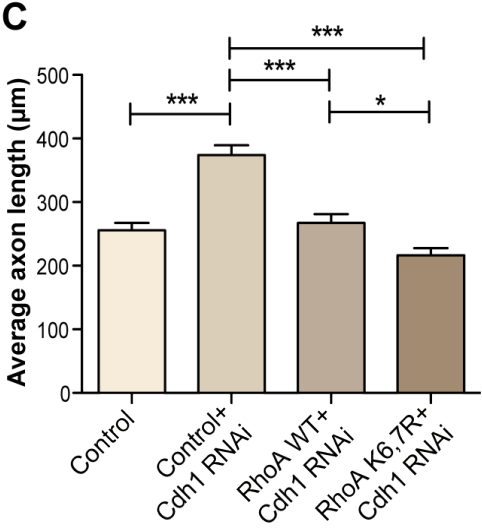

Figure 3.28 Smurf1 promotes axon growth by degrading RhoA. A. Neurons were transfected with control vectors pCMV5 and pCEFL, or Smurf1 DBM3/4 expression plasmid together with pCEFL or plasmids encoding RhoA WT or RhoA K6,7R, maintained in conditioned medium and analyzed at DIV 3 as described in Figure 3.3. A total of 605 neurons was measured (ANOVA, ${ }^{* * *} \mathrm{p}<0.0001,{ }^{*} \mathrm{p}<0.05$, values indicate mean +SEM). B. Granule neurons were transfected at DIV 0 with control vectors pCMV5 and pCEFL, or Smurf1 DBM3/4 expression plasmid together with pCEFL or plasmids encoding RhoA WT or Cdc42 WT, maintained in conditioned medium and analyzed at DIV 3 as in Figure 3.3. A total of 289 neurons was measured (ANOVA, $* * * \mathrm{p}<0.0001$, n.s. non-significant, values indicate mean $+\mathrm{SEM}$ ). C. Neurons were transfected with control vectors U6 and pCEFL, or Cdh1 RNAi plasmid together with pCEFL or plasmids encoding RhoA WT or RhoA K6,7R, maintained in conditioned medium and analyzed at DIV 3 as in Figure 3.3. A total of 646 neurons was measured (ANOVA, ${ }^{* * *} \mathrm{p}<0.0001,{ }^{*} \mathrm{p}<0.05$, values indicate mean + SEM).

To address whether Cdh1-deficiency affects global levels of total or active RhoA, brain lysates of adult $\mathrm{Cdh} 1+/+$ and $\mathrm{Cdh} 1+/-$ mice were subjected to immunoblotting using a RhoA antibody for total RhoA, and to a GST-pulldown assay using GST-Rhotekin sepharose beads followed by RhoA immunoblotting for active RhoA. Activated RhoA binds its effector Rhotekin (Reid et al., 1996) thus, GST-tagged Rhotekin specifically associates with RhoAGTP in pulldown assays (Ren et al., 1999; Sander et al., 1998). Neither total nor active RhoA levels are affected in the Cdh1+/- mice (Figure 3.29A) suggesting that global RhoA levels are not altered under Cdh1-deficiency. However, given that Smurfl levels are locally regulated in axon initiation (Cheng et al., 2011), I reasoned that RhoA levels may also be similarly locally modulated by Cdh1-APC. Thus, I transfected hippocampal neurons at DIV 2 with control vector or the Cdh1 RNAi plasmid together with GFP plasmid and at DIV 6 subjected the neurons to double-immunostaining using RhoA and GFP antibodies. After appropriate background subtraction, mean grey values of RhoA and GFP staining were obtained at axon 
tips and at a proximal region in the axon as reference. RhoA values were normalized to that of GFP in both regions of interest and further normalized to each other. The analysis revealed that total RhoA levels are significantly reduced at axon tips under Cdh1 knockdown as compared to control neurons (Figure 3.29B, C). In sum, a loss of Cdh1 reduces local but not global levels of RhoA.

A

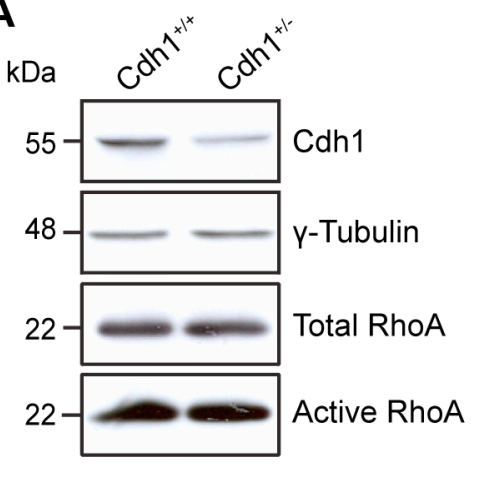

B

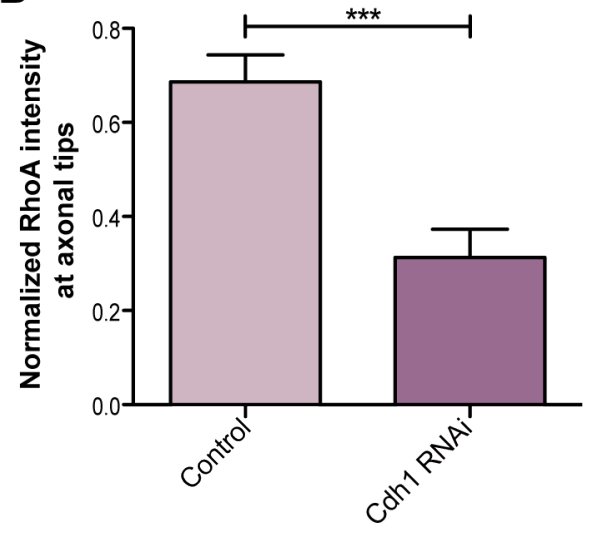

C
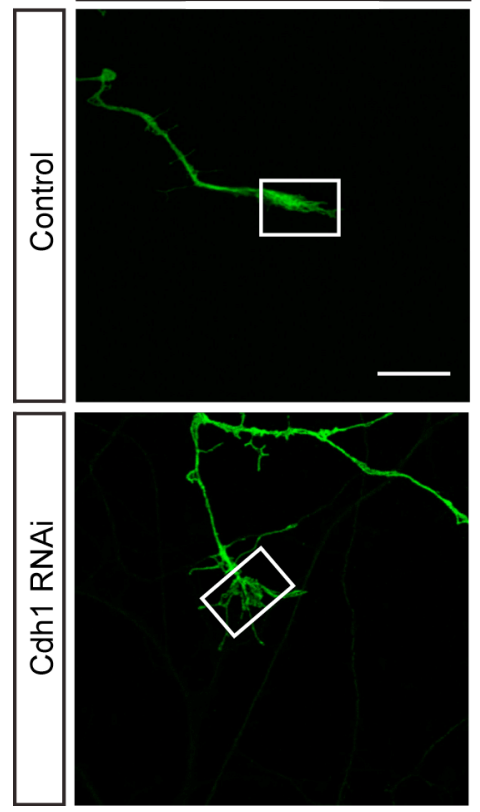
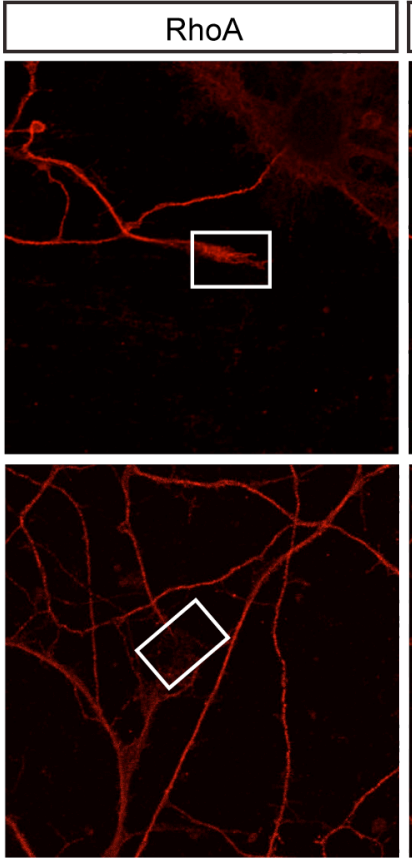

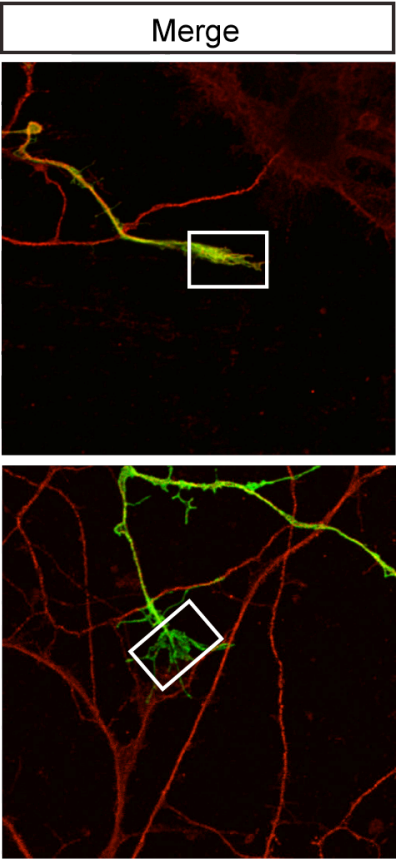

Figure 3.29 Cdh1 knockdown affects local RhoA levels. A. Cerebellar lysates of W10 (week 10) $\mathrm{Cdh}^{+/+}$and $\mathrm{Cdh}^{+/-}$mice were immunoblotted with the RhoA and Cdh1 antibodies. $\gamma$-tubulin served as loading control. For detection of active RhoA, the lysates were subjected to GST-pulldown using GST-Rhotekin conjugated beads and immunoblotted using the RhoA antibody. GST-pulldown was reproduced by Shih-Ju Lee, Stegmüller Lab, MPIEM, Göttingen. B. Hippocampal neurons transfected at DIV 2 with control U6 or Cdh1 RNAi plasmid together with the GFP plasmid were fixed at DIV 6 and subjected to double-immunostaining using the GFP and RhoA antibodies and Hoechst 33258. The intensity of RhoA staining at axon tips and axons was determined by the mean grey values and normalized to that of GFP. Normalized RhoA intensity at the axon tips was further normalized to that in the axons C. Representative images of transfected neurons in B. A total of 28 neurons was measured (t-test, $* * * p<0.0001$, values indicate mean $+\mathrm{SEM})$. Box indicates region of interest. Scale bar equals $5 \mu \mathrm{m}$. 


\subsubsection{Smurf1 DBM3/4 protects neurons from the growth inhibitory effects of myelin}

Myelin is a major extracellular growth inhibitory component of the nervous system that is implicated in the inhibition of CNS regeneration after injury and disease (McKerracher et al., 1994; Mukhopadhyay et al., 1994; Schwab et al., 1993). Myelin proteins exert their inhibitory effect by binding to the neuronal Nogo-66 receptor complex and eliciting downstream cascades that converge on the small GTPase RhoA (McGee and Strittmatter, 2003; Schwab, 2004; Yiu and $\mathrm{He}, 2006)$. It has been previously demonstrated that Cdh1 knockdown overrides myelin inhibition of axon growth (Konishi et al., 2004). Given that RhoA mediates the effect of Cdh1 in axon growth inhibition and that myelin-triggered growth inhibitory cascades meet on RhoA, I envisaged a role for Smurf1 DBM3/4, a Cdh1-degradation resistant mutant and a negative regulator of RhoA in counteracting myelin inhibition of axon growth.

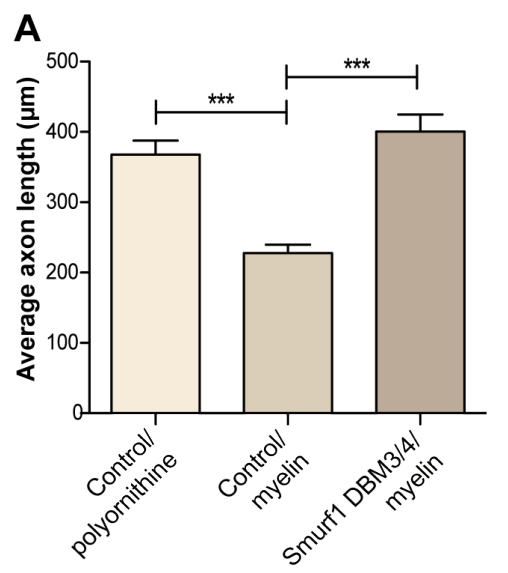

B
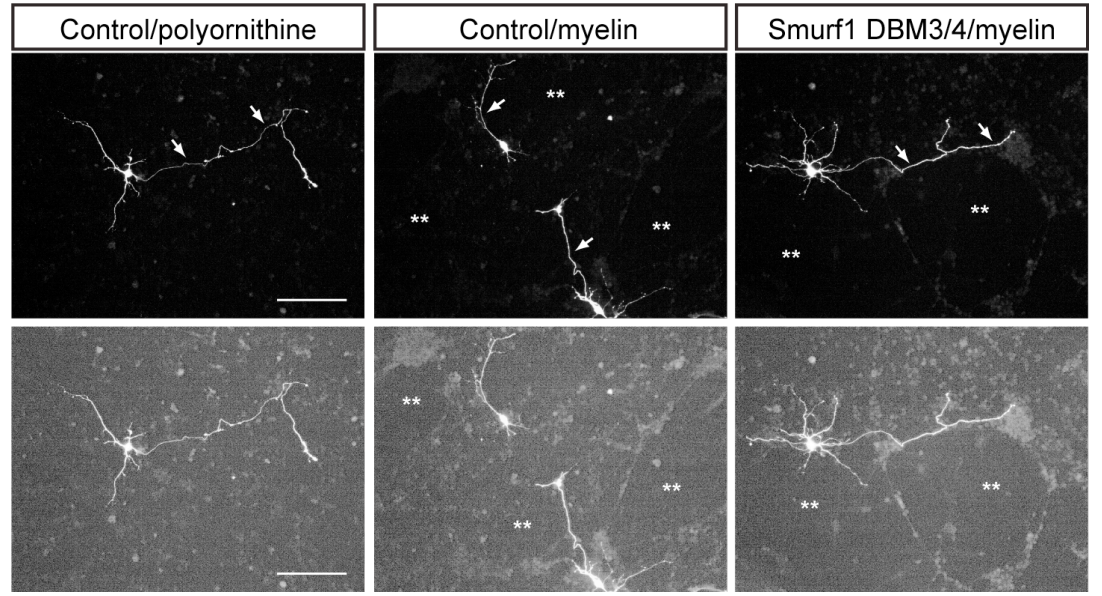

Figure 3.30 Smurf1 DBM3/4 promotes axon growth on myelin. A. Cerebellar granule neurons cultured on polyornithine or polyornithine+myelin (referred to as myelin) were transfected at DIV 0 with control pCMV5 or Smurf1 DBM3/4 plasmid, maintained in conditioned medium and analyzed at DIV 4 as in Figure 3.3. A total of 414 neurons was measured (ANOVA, $* * * p<0.0001$, values indicate mean + SEM). B. Upper panel, representative images of transfected neurons in A. Scale bar equals $100 \mu \mathrm{m}$. Arrows indicate axons. Lower panel, high contrast images showing myelin rich regions (indicated by asterisks) with low neuron density.

To test this hypothesis, granule neurons plated at DIV 0 on the permissive substrate polyornithine were transfected with the control vector pCMV5 and neurons plated on the inhibitory substrate myelin were transfected with either pCMV5 or Smurf1 DBM3/4. Axon assays were performed at DIV 4. The inhibitory effect of myelin on axon growth is evident at this stage since control neurons on myelin possess significantly shorter axons than those on polyornithine. Moreover, neurons on myelin tend to be strongly repelled by myelin-rich regions and grow in clusters of neuronal cell bodies. However, this inhibitory effect of myelin is not observed in neurons transfected with Smurf1 DBM3/4, which possess axons that are 
significantly longer than the control neurons on myelin and are in fact, of similar lengths to control neurons on polyornithine (Figure 3.30). Thus, Smurf1 DBM3/4 may serve to overcome myelin inhibition of axon growth presumably by modulating the activity of RhoA, which is also the major downstream component of myelin signaling.

\section{4 p250GAP, a Rho GTPase activating protein, promotes axon growth downstream of Cdh1-APC}

\subsection{1 p250GAP, a brain-enriched RhoGAP is a novel interactor of $\mathrm{Cdh} 1$}

Besides Smurf1, RhoA activity is tightly regulated by Rho Guanine nucleotide Exchange Factors (GEFs) and Rho GTPase Activating Proteins (GAPs), respectively. I hypothesized that a RhoGAP, a negative Rho regulator, may be a potential Cdh1-APC substrate in axon growth. Thus, RhoGAPs that are brain-abundant and are implicated in neuronal morphogenesis (Appendix 4) were tested for their interaction with Cdh1 in a series of coimmunoprecipitation analyses (Appendix 4). Among the RhoGAPs analyzed, p250GAP, a RhoA/Cdc42 GAP (Nakazawa et al., 2008; Nakazawa et al., 2003) emerged as a novel interactor of Cdh1. Lysates of heterologous cells transfected with control and Cdh1, control
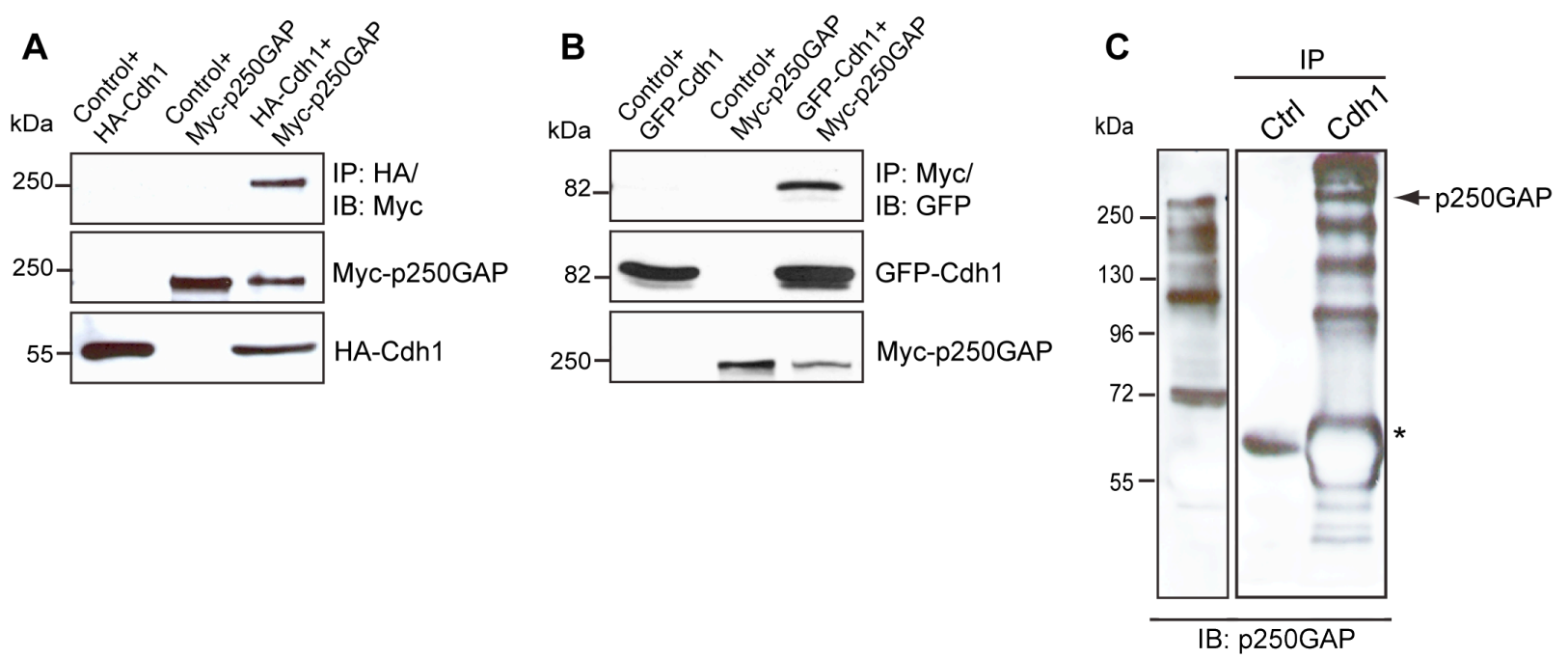

Figure 3.31 p250GAP interacts with Cdh1. A. Lysates of 293T cells transfected with control vector pCDNA3 and HA-Cdh1, or control pCMV5 vector and Myc-p250GAP, or HA-Cdh1 and Myc-p250GAP plasmids were subjected to immunoprecipitation with the HA antibody and immunoblotting with the Myc antibody. B. Lysates of 293 T cells transfected with control vector pCDNA3 and GFP-Cdh1, or GFP and Myc-p250GAP plasmid, or plasmids encoding GFP-Cdh1 and Myc-p250GAP were subjected to immunoprecipitation with the Myc antibody followed by immunoblotting with the GFP antibody. C. P9 mouse brain lysates were subjected to immunoprecipitation with control serum or Cdh1 antiserum followed by immunoblotting with p250GAP polyclonal antibody. Arrow indicates p250GAP and asterisk, $\operatorname{IgG}_{\mathrm{H}}$.

and p250GAP or both Cdh1 and p250GAP expression plasmids were subjected to coimmunoprecipitation assays. Exogenous p250GAP interacts robustly with Cdh1 (Figure 3.31A, 
B). In addition, p250GAP and Cdh1 form a physical complex in the mammalian brain (Figure $3.31 \mathrm{C})$.
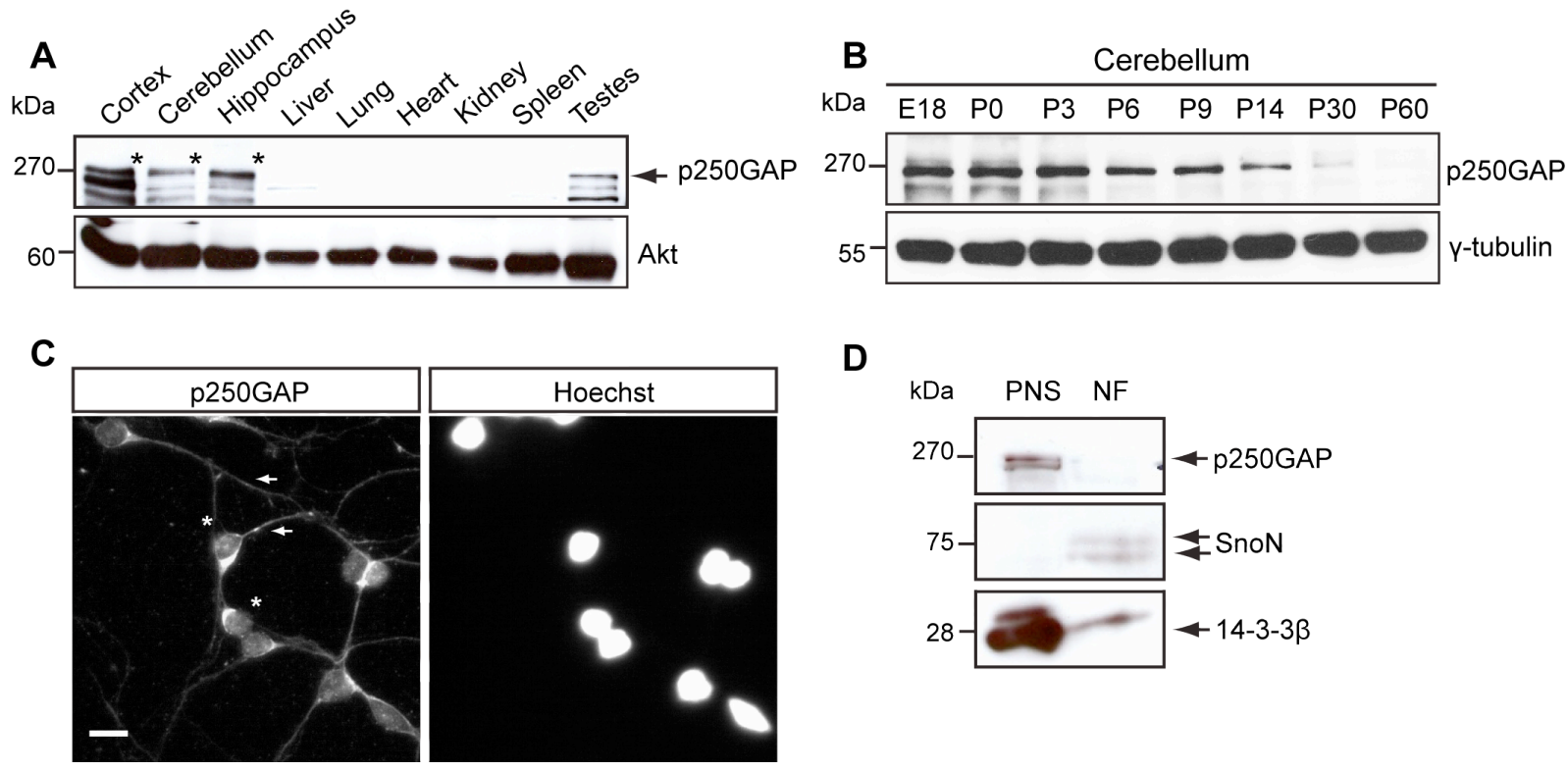

D

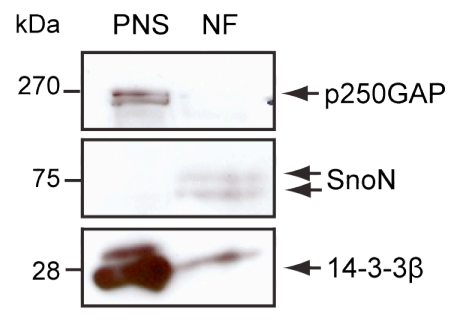

Figure 3.32 p250GAP is brain-abundant and localizes to the cytoplasm in neurons. A. Lysates of indicated tissues collected from wild type P60 mouse were subjected to immunoblotting using the p250GAP antibody. Akt served as loading control. Asterisks indicate p250GAP. B. Cerebella were collected from wild type rats at the indicated days and lysates were subjected to immunoblotting using the p250GAP antibody. $\gamma$-tubulin served as loading control. $\mathrm{E}=$ embryonic, $\mathrm{P}=$ postnatal. C. Granule neurons at $\mathrm{DIV} 2$ were subjected to immunocytochemistry with the p250GAP antibody and the nuclear dye bisbenzimide Hoechst 33258 . Arrows and asterisk indicate axons and soma, respectively. Scale bar equals $10 \mu \mathrm{m}$. D. Cortical neurons were subjected to subcellular fractionation. Nuclear (NF) and postnuclear supernatant (PNS) fractions were immunoblotted using p250GAP, SnoN and 14-3-3 $\beta$ antibodies. SnoN and 14-3-3 $\beta$ served as controls for NF and PNS, respectively. Subcellular fractionation was performed by Dr. Judith Stegmüller, MPI-EM, Göttingen.

Consistent with previous findings (Nakazawa et al., 2003), I found that p250GAP is brain-enriched in the adult mouse (Figure 3.32A). To examine the temporal expression profile of p250GAP, cerebella were collected from rat at different ages and the lysates were immunoblotted with p250GAP polyclonal antibody. p250GAP expression is high in late embryonic and early postnatal brain (E18, P0-P14), but its levels decrease over time (Figure 3.32B).

To identify the subcellular localization of p250GAP, granule neurons were subjected to immunocytochemistry using the p250GAP antibody. p250GAP localizes to the cytoplasm and processes in cultured neurons (Figure 3.32C). The cytoplasmic localization of the RhoGAP was further confirmed using subcellular fractionation analyses. Subcellular fractionation was performed by Dr. Judith Stegmüller, MPI-EM, Göttingen. p250GAP segregates entirely to the postnuclear supernatant fraction (Figure 3.32D). Taken together, my data suggest that 
p250GAP, a novel Cdh1-interactor is a brain-abundant RhoGAP and at the subcellular level, localizes to the cytoplasm where it may modulate RhoA activity.

\subsubsection{Knockdown of p250GAP reduces axon growth in cerebellar granule neurons}

In order to study the function of p250GAP in axon growth regulation, I triggered an acute knockdown of p250GAP in granule neurons and performed functional assays. A previously identified target region (Nakazawa et al., 2008) was used to generate a p250GAP RNAi plasmid and its p250GAP knockdown effect was validated in heterologous cells. 293T cells were transfected with control vector or p250GAP RNAi plasmid together with full-length p250GAP and analysis of lysates confirmed the efficient knockdown of p250GAP by the RNAi construct (Figure 3.33A).
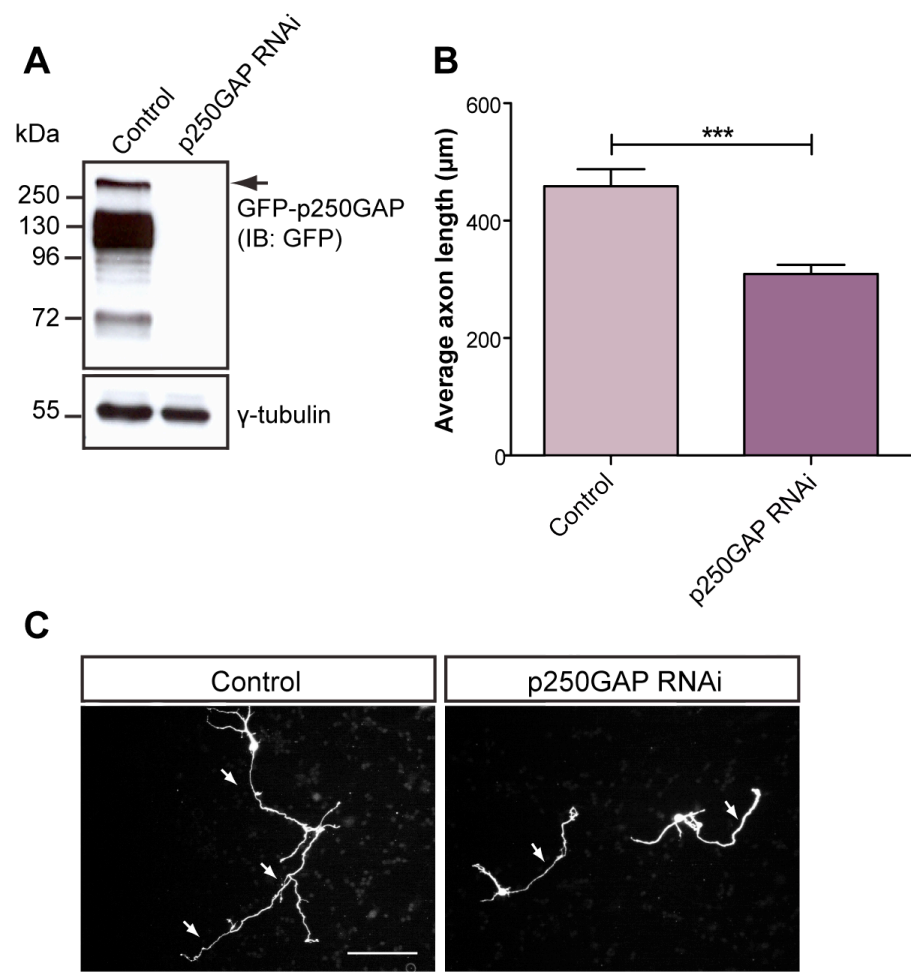

Figure 3.33 p250GAP promotes axon growth. A. 293T cells were transfected with a plasmid encoding GFPp250GAP together with control vector U6 or the p250GAP RNAi plasmid. Lysates were subjected to immunoblotting using the GFP antibody. $\gamma$-tubulin served as the loading control. B. Cerebellar granule neurons were transfected at DIV 0 with control vector U6 or the p250GAP RNAi plasmid and maintained in conditioned medium. At DIV 4, neurons were analyzed as in Figure 3.3. A total of 300 neurons was measured (t-test, $* * * \mathrm{p}<0.0001$, values indicate mean $+\mathrm{SEM})$. C. Representative images of transfected neurons in B. Scale bar equals $100 \mu \mathrm{m}$. Arrows indicate axons.

Next, cultured granule neurons were transfected at DIV 0 with either control or the p250GAP RNAi plasmid and subjected to axon growth assays at DIV 4. Knockdown of p250GAP results in a significant decrease in axon length (Figure 3.33B, C). 


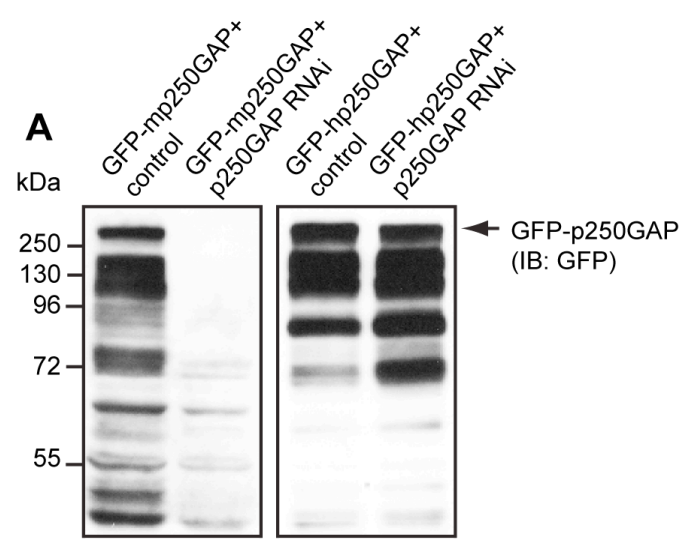

B
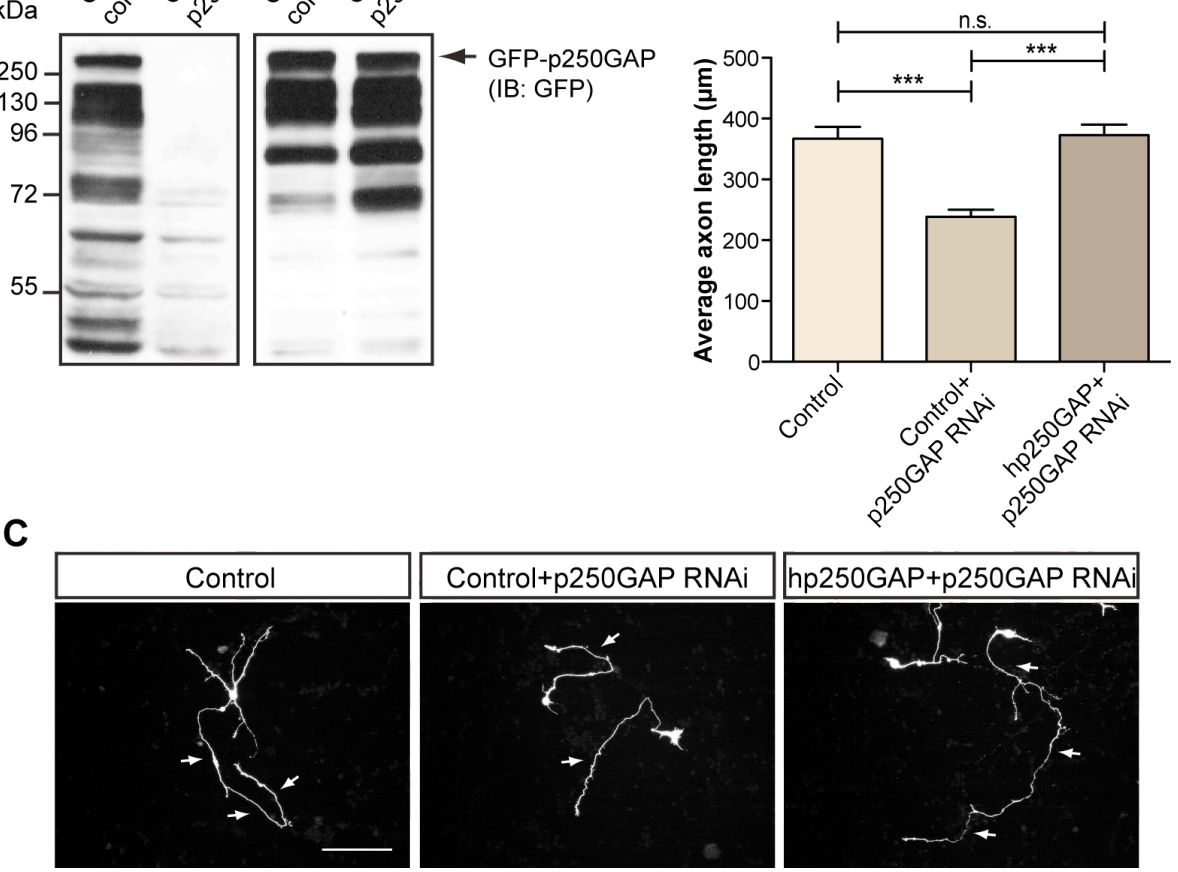

Figure 3.34 A rescue plasmid reverts p250GAP knockdown phenotype. A. Lysates of 293T cells transfected with GFP-tagged mouse (mp250GAP) or human (hp250GAP) p250GAP together with control vector U6 or mouse/rat-specific p250GAP RNAi plasmid were immunoblotted using the GFP antibody. B. Granule neurons were transfected at DIV 0 with control vectors pCDNA3 and U6, or p250GAP RNAi together with pCDNA3 or human p250GAP plasmids and maintained in conditioned medium. At DIV 4, neurons were analyzed as in Figure 3.3. A total of 273 neurons was measured (t-test, ${ }^{* * *} \mathrm{p}<0.0001$, n.s. $=$ not significant, values indicate mean $+\mathrm{SEM}$ ). C. Representative images of transfected neurons in B. Scale bar equals $100 \mu \mathrm{m}$. Arrows indicate axons.

To determine whether the p250GAP-induced axonal phenotype is the result of a specific knockdown of p250GAP, rescue assays were performed using a plasmid encoding human p250GAP that harbors several silent mutations in the stretch corresponding to the rodent targeting region. In $293 \mathrm{~T}$ cells, the rodent-specific RNAi fails to induce knockdown of human p250GAP (Figure 3.34A). In granule neurons, expression of human p250GAP together with rodent p250GAP RNAi significantly restores axonal length to baseline length in control neurons (Figure 3.34B, C). In summary, my results identify p250GAP as an axon growth promoting protein.

\subsection{3 p250GAP is not degraded in a proteasome-dependent manner and its protein levels do not accumulate in the Cdh1-heterozygotes}

Owing to its interaction with Cdh1 and its axon growth-promoting effect, I asked if p250GAP is a target of Cdh1-APC in the control of axonal morphogenesis. To test if p250GAP is degraded in a proteasome-dependent manner, granule neurons were treated with vehicle or 
lactacystin for $10 \mathrm{~h}$ and p250GAP expression was probed for in the lysates. In contrast to Smurf1, p250GAP does not accumulate on proteasome inhibition (Figure 3.35A). The lack of response of p250GAP to proteasome inhibition was additionally confirmed by treating neurons with $10 \mathrm{nM}$ bortezomib, another highly specific inhibitor of the $26 \mathrm{~S}$ proteasome. Lysates were simultaneously immunoblotted for Smurf1, a positive control (Figure 3.35B). Further, to explore whether p250GAP levels respond to reduced Cdh1-APC activity, p250GAP expression was evaluated in brain lysates from $\mathrm{Cdh}^{+/+}$and $\mathrm{Cdh}^{+/-}$mice. I found that $\mathrm{p} 250 \mathrm{GAP}$ expression is invariable between the wild-type and $\mathrm{Cdh}^{+/-}$mice brains (Figure 3.36A) while the positive control, SnoN, a known target of Cdh1-APC (Stegmuller et al., 2006), accumulates in the heterozygotes (Figure 3.3B).

A

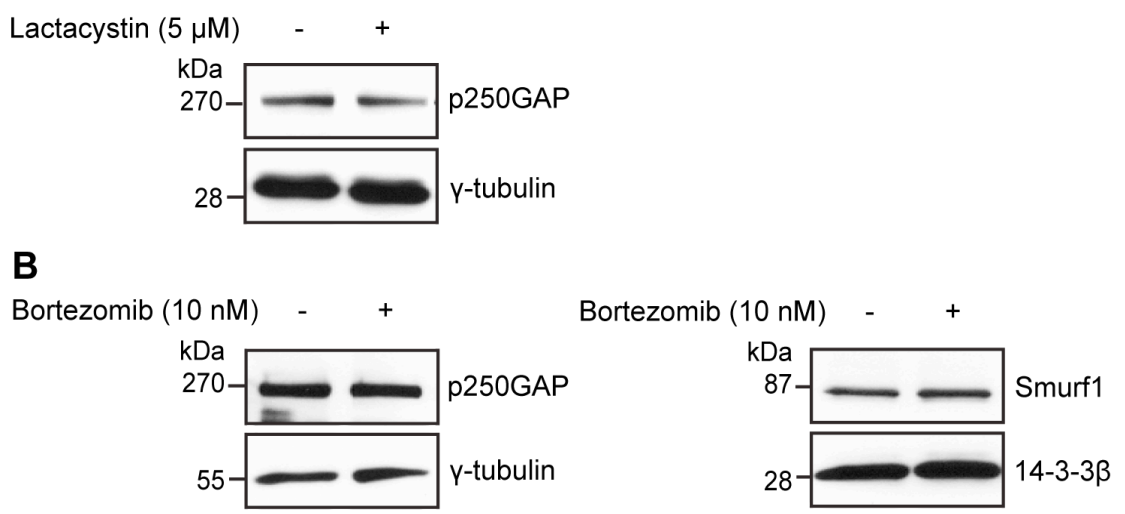

Figure 3.35 p250GAP is unresponsive to proteasome inhibition. A. Lysates of granule neurons treated with vehicle or $5 \mu \mathrm{M}$ lactacystin for $10 \mathrm{~h}$ were subjected to immunoblotting using the p250GAP antibody. 14-3-3 $\beta$ served as the loading control. B. Lysates of granule neurons treated with vehicle or $10 \mathrm{nM}$ bortezomib for $10 \mathrm{~h}$

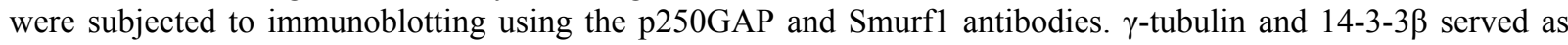
loading controls, respectively.

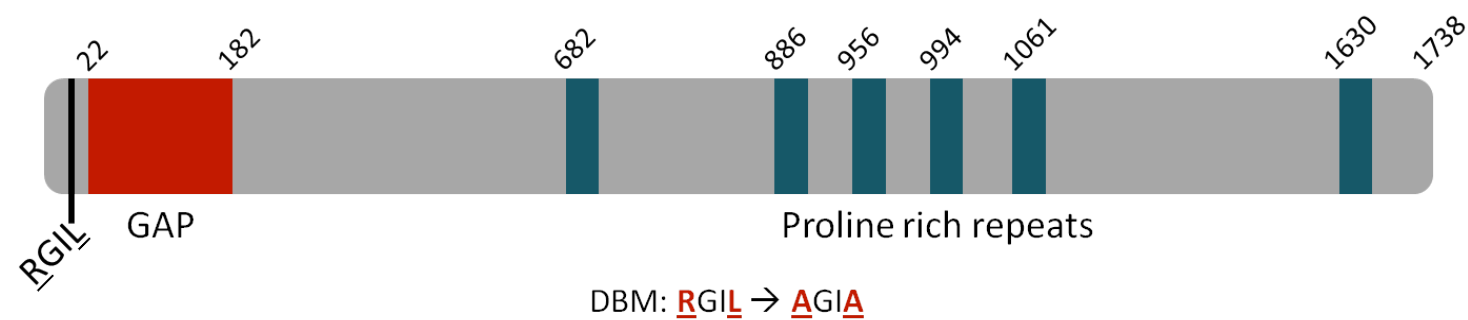

Schematic 3B. Putative D-box in p250GAP. Human p250GAP sequence consists of 1738 amino acids with a GAP domain at its N-terminus and proline-rich motifs towards the C-terminus. The sequence harbors one putative D-box at its N-terminus. In the generation of the D-box mutant, the conserved residues $\mathrm{R}$ and $\mathrm{L}$ were mutated to alanine. 

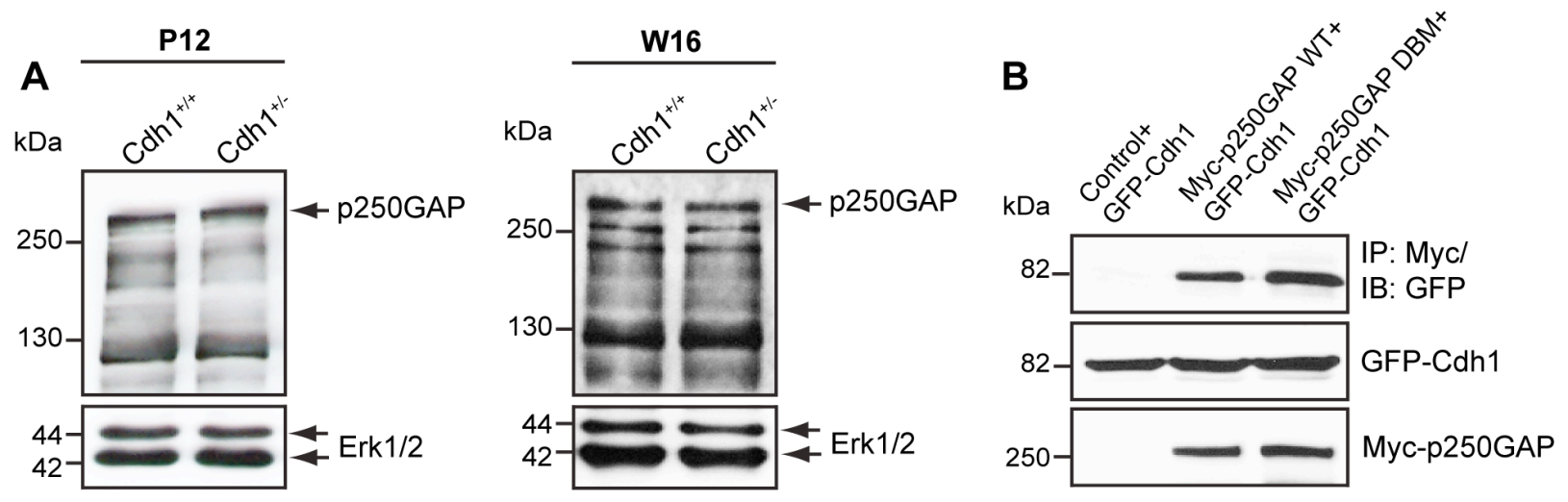

Figure 3.36 p250GAP levels are unaffected by Cdh1-APC. A. Cerebellar lysates of P12 and W16 Cdh1 $1^{+/+}$and $\mathrm{Cdh}^{+/-}$mice were immunoblotted using the p250GAP antibody. Erk1/2 served as loading control. B. Lysates of 293 T cells transfected with GFP-Cdh1 plasmid together with control vector pCDNA3, Myc-p250GAP wild-type (Myc-p250GAP WT) or the D-box mutant (Myc-p250GAP DBM) were subjected to immunoprecipitation using the Myc antibody followed by immunoblotting with the GFP antibody.

Moreover, mutation of a conserved N-terminal $\underline{R} G \underline{L} \underline{L}$ sequence (Schematic 3B), the only putative Cdh1-recognition site in p250GAP, neither stabilizes the protein nor interferes with its binding to Cdh1 in co-immunoprecipitation assays in heterologous cells (Figure 3.36B). In essence, the results indicate that p250GAP is not targeted for proteasomal degradation by Cdh1-APC.

\subsection{4 p250GAP undergoes non-proteolytic ubiquitination at its C-terminus}

Since my data suggest that p250GAP is not degraded by the proteasome, I asked whether p250GAP undergoes a non-proteolytic but dynamic activity-modifying ubiquitination. Covalent attachment of ubiquitin to substrates has been previously shown to also modulate their function or mediate cellular processes such as receptor internalization, sorting or transcriptional regulation without eliciting proteolytic degradation (Hicke, 2001; Welchman et al., 2005). Di-ubiquitination of the Ras GTPase HRas has been shown to stabilize its association with the endosome and enhance its ability to trigger downstream Raf/MAPK signaling (Jura et al., 2006). In a neuronal context, inactivation of the small GTPase Rap2A by Nedd 4-1-mediated mono-ubiquitination has been implicated in the control of dendritic growth (Kawabe et al., 2010).

To test the hypothesis of non-proteolytic ubiquitination of p250GAP, p250GAP fusion constructs were generated in which a ubiquitin moeity was appended to the $\mathrm{N}$ - and $\mathrm{C}$ - terminus of the protein resulting in ubiquitin-p250GAP and p250GAP-ubiquitin, respectively. Previous work on ubiquitin fusion proteins has shown that proteins carrying a non-removable ubiquitin moiety at their $\mathrm{N}$-terminus or in the middle of their sequence are proteolytically degraded 
whereas C-terminal ubiquitin conjugates are metabolically stable (Johnson et al., 1995; Qian et al., 2002).
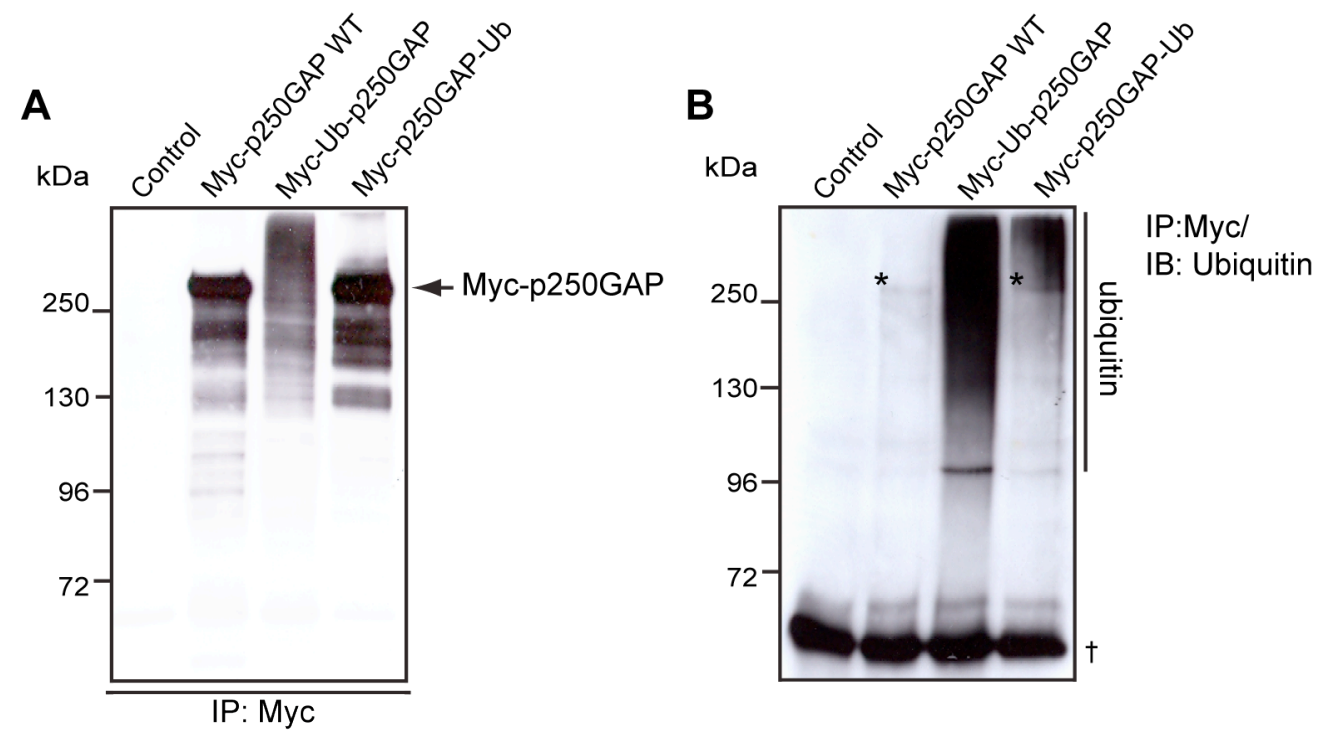

Figure 3.37 p250GAP-ubiquitin is not de-stabilized. A. Lysates of 293T cells transfected with pCDNA3 control vector, Myc-p250GAP wild-type (Myc-p250GAP WT), Myc-ubiquitin-p250GAP (Myc-Ub-p250GAP) or Mycp250GAP-ubiquitin (Myc-p250GAP-Ub) plasmid were subjected to immunoblotting using the Myc antibody. B. Lysates of 293T transfected with pCDNA3 control vector, Myc-p250GAP WT, Myc-ubiquitin-p250GAP or Mycp250GAP-ubiquitin plasmid were subjected to immunoprecipitation using the Myc antibody followed by immunoblotting with ubiquitin antibody. Asterisks indicate ubiquitinated p250GAP, ' $\dagger$ ' indicates $\operatorname{IgG}_{\mathrm{H}}$.

In preliminary experiments, I found that ubiquitin-p250GAP is highly unstable and produces a smear in Western blot analysis indicative of potential poly-ubiquitination and degradation while p250GAP-ubiquitin is as stable as the wild-type form (Figure 3.37A). Next, lysates of 293 T cells expressing Myc-tagged p250GAP WT, ubiquitin-p250GAP or p250GAPubiquitin plasmids were subjected to immunoprecipitation using the Myc antibody followed by immunoblotting with ubiquitin antibody. While very little ubiquitin is associated with p250GAP WT resulting in a narrow band, p250GAP-ubiquitin produces a similar band and a slight high molecular weight smear above $250 \mathrm{kDa}$. However, ubiquitin-p250GAP produces an intense poly-ubiquitination and degradation smear (Figure 3.37B). Given that p250GAP does not respond to proteasome inhibition (Figure 3.35) and that the ubiquitin-p250GAP fusion protein is destabilized, it is very unlikely that endogenous p250GAP undergoes N-terminal ubiquitination. Taken together, my experiments indicate that p250GAP undergoes nonproteolytic ubiquitination close to or at its C-terminus. 

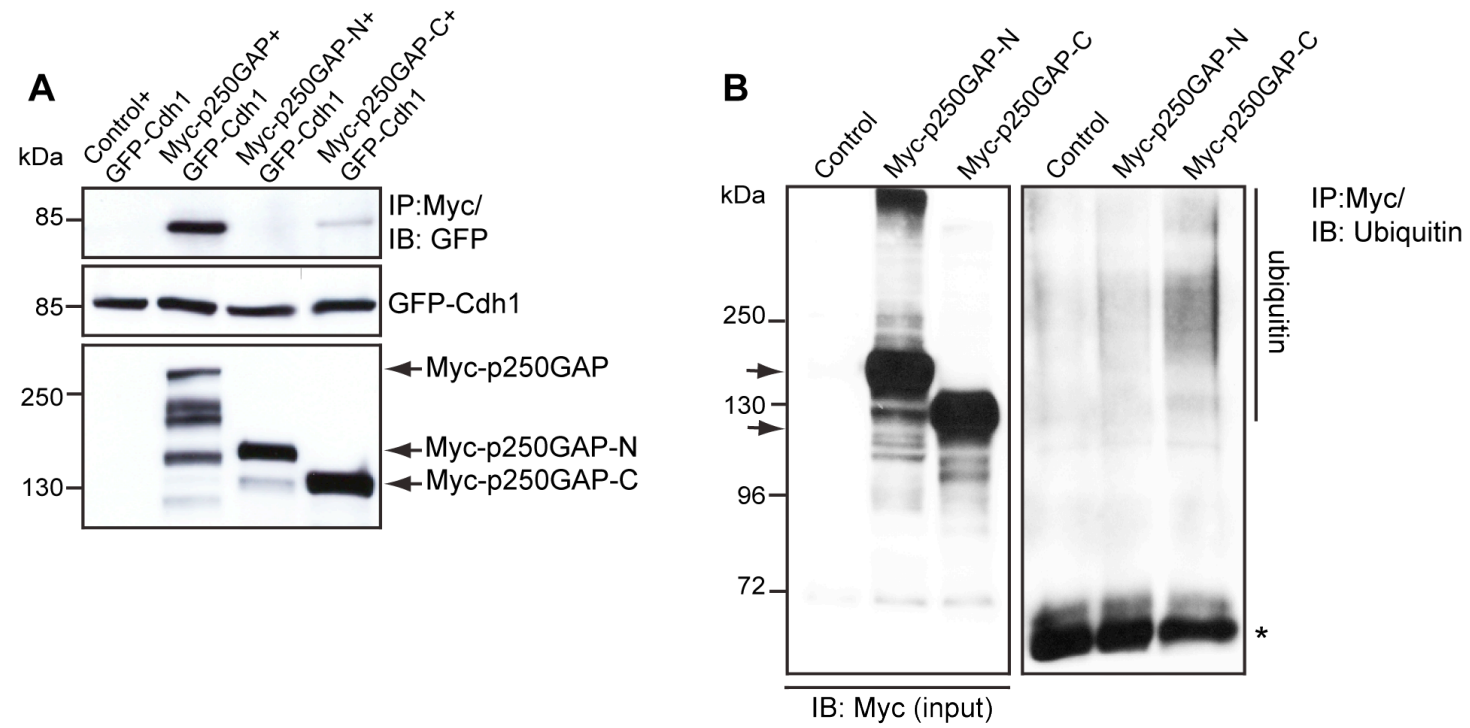

Figure 3.38 C-terminal fragment of p250GAP interacts with Cdh1. A. Lysates of 293T cells transfected with GFP-Cdh1 together with control vector pCMV-Myc, or plasmids encoding Myc-tagged full length, N- or Cterminal fragments of p250GAP (Myc-p250GAP, Myc-p250GAP-N or Myc-p250GAP-C) were subjected to immunoprecipitation with the Myc antibody followed by immunoblotting with the GFP antibody. B. Lysates of 293 T cells transfected with control vector pCMV-Myc, Myc-p250GAP, Myc-p250GAP-N or Myc-p250GAP-C plasmid were immunoprecipitated using the Myc antibody followed by immunoblotting with ubiquitin antibody. Asterisk indicates $\operatorname{IgG}_{\mathrm{H}}$.

To corroborate my results, I tested the interaction of Cdh1 with $\mathrm{N}$ - and C-terminal fragments of p250GAP in coimmunoprecipitation assays in HEK 293T cells. Lysates of 293T cells transfected with Myc-tagged full-length, N- (1-860 AA) or C-terminal (861-1738 AA) fragments of p250GAP together with GFP-Cdh1 were subjected to immunoprecipitation with the Myc antibody followed by immunoblotting with the GFP antibody. I found that Cdh1 interacts specifically with the C-terminal fragment of p250GAP (Figure 3.38A). Besides, ubiquitination assays using lysates of $293 \mathrm{~T}$ expressing $\mathrm{N}$ or C-terminal p250GAP reveal robust ubiquitination of the C-terminal fragment while p250GAP-N is associated with less ubiquitin (Figure 38B). Collectively, my results suggest the involvement of p250GAP C-terminus in its interaction with Cdh1 and subsequent non-destabilizing ubiquitination.

\subsubsection{C-terminally ubiquitinated p250GAP is stable but fails to promote axon growth}

I next set out to investigate whether C-terminal ubiquitination modifies the activity of p250GAP in the control of axon growth. In a similar rescue experiment as described in section 3.4.2, cultured granule neurons were transfected at DIV 0 with control or p250GAP RNAi plasmid together with p250GAP WT, ubiquitin-p250GAP or p250GAP-ubiquitin expression plasmids and axon lengths were analyzed at DIV 4. While there is again a rescue of axon length upon expression of p250GAP WT in the background of p250GAP knockdown, neither 
ubiquitin-p250GAP nor p250GAP-ubiquitin reverts the p250GAP knockdown phenotype (Figure 3.39).

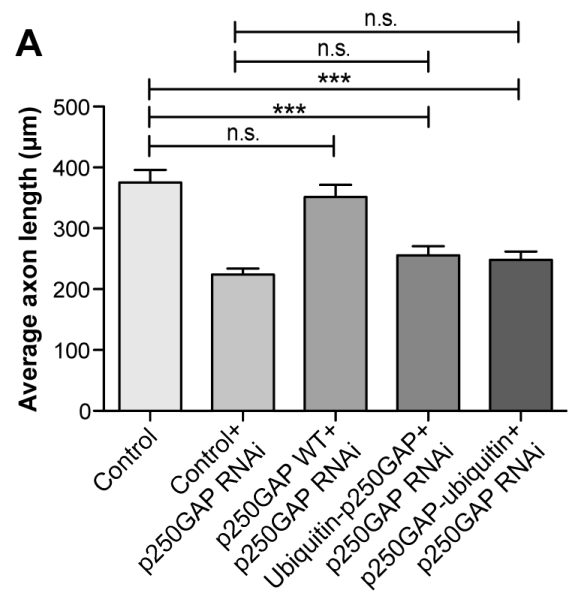

B
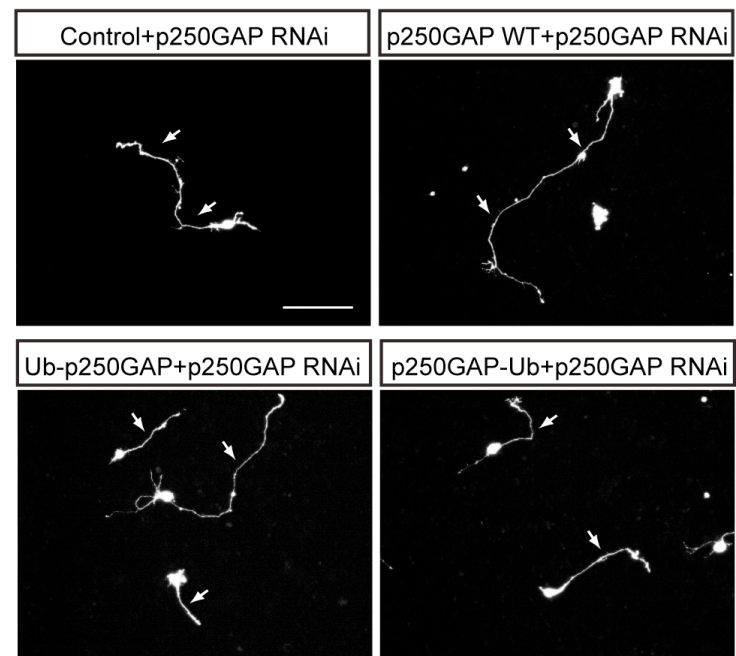

Figure 3.39 p250GAP-ubiquitin exhibits a loss-of-function in axon growth. A. Granule neurons were transfected at DIV 0 with the control vector pCDNA3 together with U6 or the p250GAP RNAi plasmid, or p250GAP RNAi together with p250GAP WT, Ubiquitin-p250GAP (Ub-p250GAP) or p250GAP-ubiquitin (p250GAP-Ub) plasmids and maintained in conditioned medium. At DIV 4, neurons were analyzed as in Figure 3.3. A total of 533 neurons was measured (ANOVA, ${ }^{* * *} \mathrm{p}<0.0001$, n.s. $=$ not significant, values indicate mean +SEM). B. Representative images of transfected neurons in A. Scale bar equals $100 \mu \mathrm{m}$. Arrows indicate axons.

The loss-of-function of ubiquitin-p250GAP is ascribable to its destabilization while that of p250GAP-ubiquitin may imply an activity-modifying ubiquitination. The results support the notion that C-terminal ubiquitination of p250GAP does not destabilize the protein but interferes with its function in axon growth regulation. However, whether Cdh1-APC is the E3 ligase that facilitates the activity-modifying C-terminal ubiquitination of p250GAP is unclear.

\subsection{6 p250GAP regulates axon growth downstream of Cdh1-APC}

A

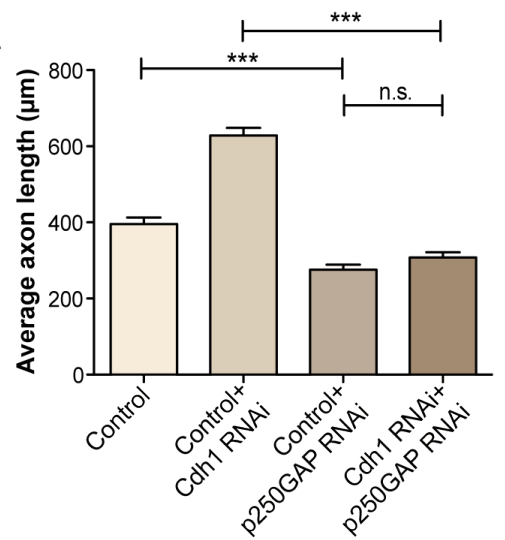

B

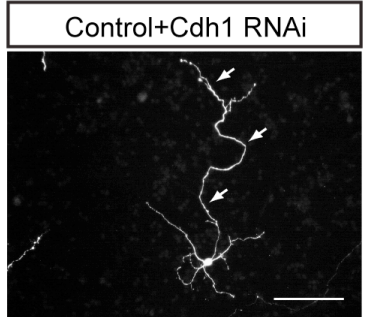

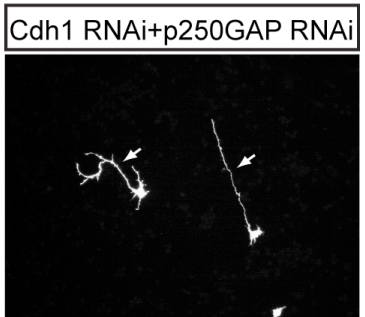

Figure 3.40 p250GAP promotes axon growth downstream of Cdh1-APC. A. Granule neurons transfected with control U6, U6 and Cdh1 RNAi, or U6 and p250GAP RNAi or both Cdh1 RNAi and p250GAP RNAi plasmids were maintained in conditioned medium and analyzed at DIV 4 as described in Figure 3.3. A total of 635 neurons was measured (ANOVA, $* * * p<0.0001$, n.s. $=$ not significant, values indicate mean + SEM). B. Representative images of transfected neurons in A. Scale bar equals $100 \mu \mathrm{m}$. Arrows indicate axons. 
To address whether p250GAP operates together with Cdh1-APC in axon growth control, epistasis experiments were performed to evaluate axon growth. Axon length was measured in Cdh1/p250GAP double knockdown neurons, Cdh1 knockdown or p250GAP knockdown neurons and compared to control neurons. Cdh1 knockdown enhances and p250GAP knockdown reduces axon length. However, neurons transfected with both RNAi plasmids display the short axon phenotype reminiscent of p250GAP knockdown alone (Figure 3.40). The results designate p250GAP as a direct downstream component of the Cdh1-APC pathway.

\subsection{7 p250GAP acts in shared pathway together with Smurf1 in axon growth regulation}

It is known that Smurfl preferentially degrades inactive RhoA in cells (Wang et al., 2003). Besides, Smurfl degradation of RhoA is a membranal event given the predominant cytosolic/membrane localization of RhoA (Michaelson et al., 2001). Thus, I speculated that a RhoGAP, which inactivates RhoA may facilitate RhoA degradation by Smurf1 in axon growth control and also serve to locally enrich Smurf1 at the membrane/growth cones, which is important for its role in axon initiation (Cheng et al., 2011). To address this possibility, I tested a potential interaction between p250GAP and Smurf1. Lysates of 293T cells transfected with control and HA-Smurf1, control and Myc-p250GAP or both HA-Smurf1 and Myc-p250GAP expression plasmids were subjected to immunoprecipitation using the Myc antibody followed by immunoblotting with the HA antibody, and vice versa. p250GAP specifically interacts with Smurf1 in heterologous cells (Figures 3.41).
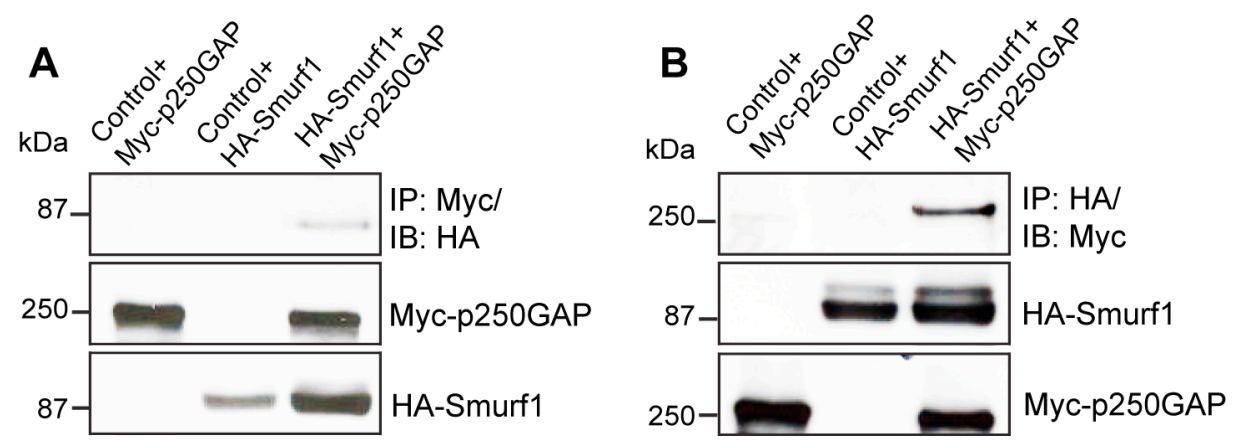

Figure 3.41 p250GAP interacts with Smurf1 in heterologous cells. A. Lysates of 293T cells transfected with control vector pCMV5 and Myc-p250GAP, or control pCDNA3 vector and HA-Smurf1, or both HA-Smurf1 and Myc-p250GAP plasmids were subjected to immunoprecipitation with the Myc antibody followed by immunoblotting with the HA antibody. B. Reciprocal co-immunoprecipitation of $\mathbf{A}$.

Next, axon assays were performed on granule neurons transfected with either p250GAP RNAi or Smurf1 RNAi plasmid together with the appropriate control vectors or with both RNAi plasmids. While both p250GAP and Smurf1 knockdown individually inhibit axon 
growth compared to control neurons, the double knockdown neurons do not display an additive effect of the RNAi-s but instead have axon lengths no different from the average axon length of the single knockdown neurons (Figure 3.42A). Additionally, to identify which of the proteins Smurfl or p250GAP exhibits the dominant phenotype, granule neurons were transfected with control vectors together with p250GAP RNAi or Smurf1 DBM3/4 or both p250GAP RNAi and Smurf1 DBM3/4 plasmids and proceeded with axon growth assays. While p250GAP RNAi inhibits axon length and Smurf1 DBM3/4 is growth-promoting, the presence of both factors results in short axons similar to p250GAP knockdown (Figure 3.42B, C). Collectively, the data indicate that p250GAP promotes axon length in a linear pathway together with Smurf1 and that the effect p250GAP is epistatic to that of Smurf1.
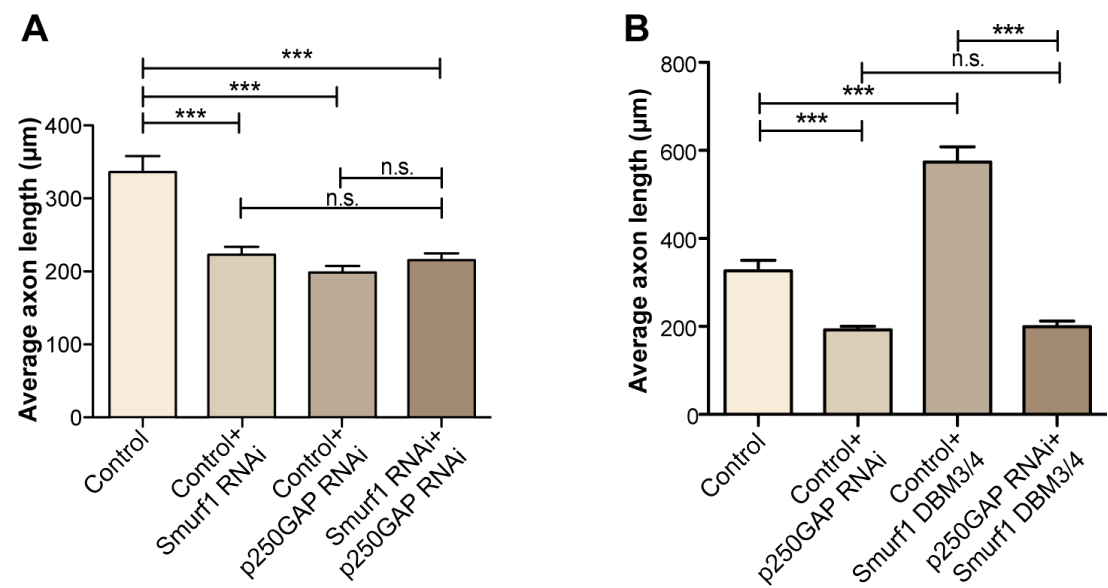

$c$
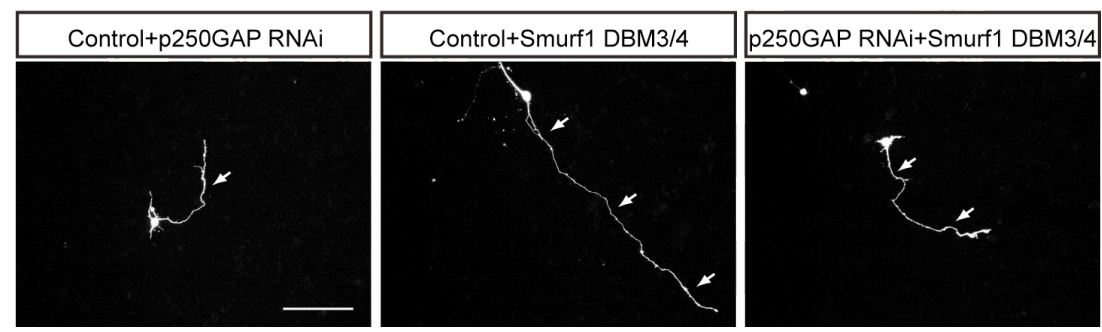

Figure 3.42 p250GAP and Smurf1 control axon growth in a shared pathway. A. Neurons transfected with control U6, U6 and p250GAP RNAi, or U6 and Smurf1 RNAi or both p250GAP RNAi and Smurf1 RNAi plasmids were maintained in conditioned medium and analyzed at DIV 4 as described in Figure 3.3. A total of 467 neurons were measured (ANOVA, ${ }^{* * *}$ p $<0.0001$, n.s. $=$ not significant, values indicate mean + SEM). B. Neurons transfected with control U6, U6 and p250GAP RNAi, U6 and Smurf1 DBM3/4 or both p250GAP RNAi and Smurf1 DBM3/4 plasmids were maintained in conditioned medium and analyzed at DIV 4 as described in Figure 3.3. A total of 324 neurons was measured (ANOVA, $* * * p<0.0001$, n.s. $=$ not significant, values indicate mean + SEM). C. Representative images of transfected neurons in B. Scale bar equals $100 \mu \mathrm{m}$. Arrows indicate axons. 


\section{5 p250GAP and Smurf1 control axon growth and migration in the developing cerebellum in vivo}

\subsubsection{Knockdown of p250GAP or Smurf1 in the developing cerebellum causes a dramatic failure of granule neurons to migrate to the internal granule layer}

To examine the function of p250GAP and Smurf1 in the developing cerebellar cortex, we carried out in vivo analyses of rat cerebella electroporated with p250GAP and Smurf1 RNAi plasmids. In vivo electroporation of rat cerebella was performed in collaboration with Dr. Judith Stegmüller and Shih-Ju Lee, MPI-EM, Göttingen. While I isolated and screened GFP-positive cerebella, fixed and embedded tissue, S.J. Lee performed cryo-sections, immunohistochemistry and analyzed the individual sections together with Dr. Stegmüller.

The control and knockdown plasmids used for in vivo electroporation are bi-cistronic constructs expressing GFP in addition to the RNAi hairpin. Plasmid DNA mix consisting of control vector U6-CMV-EGFP, p250GAP RNAi-CMV-EGFP or Smurf1 RNAi-CMV-EGFP plasmid or both knockdown plasmids together with a plasmid expressing $B C L_{X L}$, a prosurvival protein, were injected into P4 rat cerebella (Schematic 3C). The pups were subjected to repeated electric pulses. Cerebella were isolated at P9 and coronal sections were immunostained for GFP.
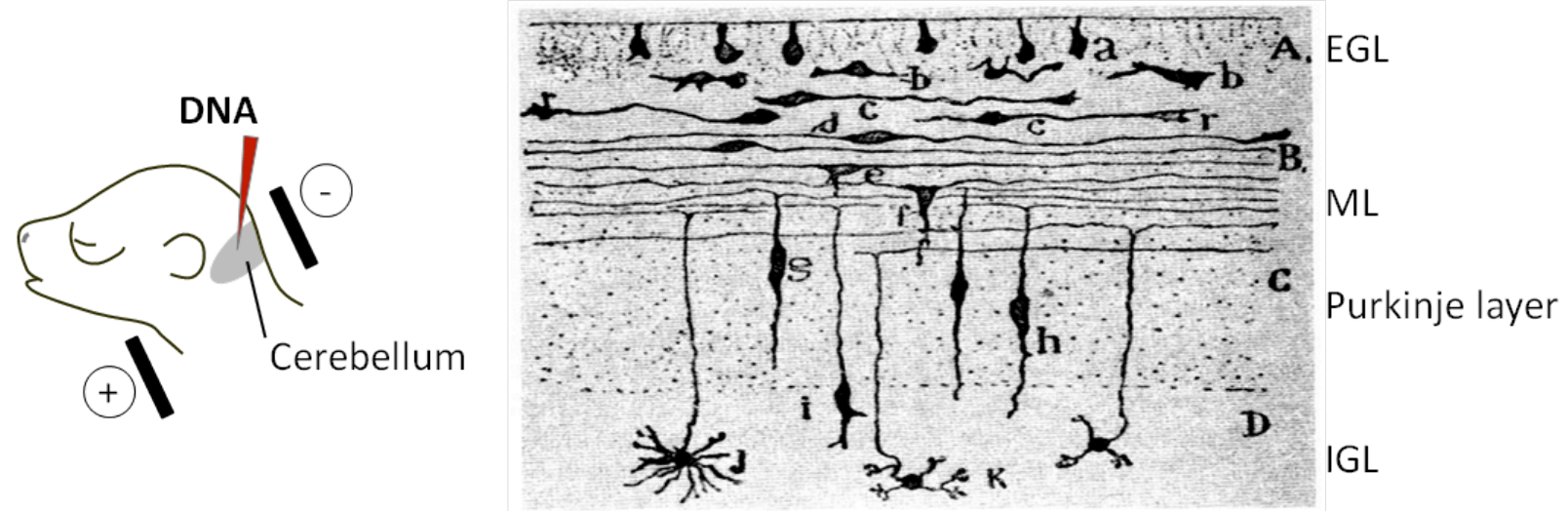

Schematic 3C. In vivo electroporation of the rat cerebellum. Left, electroporation of the cerebellum of a P4 pup. Redrawn from Konishi et al., 2004. Right, granule neuron migration during cerebellar development (Cajal, 1890). Granule neuron precursors from the germinal layers migrate to a transient external granule layer (EGL) where they develop processes. These extend bi-directionally to give rise to bi-polar axons along the molecular layer (ML). As axons continue to extend along the ML, the soma migrates longitudinally through the Purkinje layer and into the internal granule layer where it undergoes dendritic differentiation. Axons of granule neurons fasciculate to form parallel fibers characteristic of the ML. 

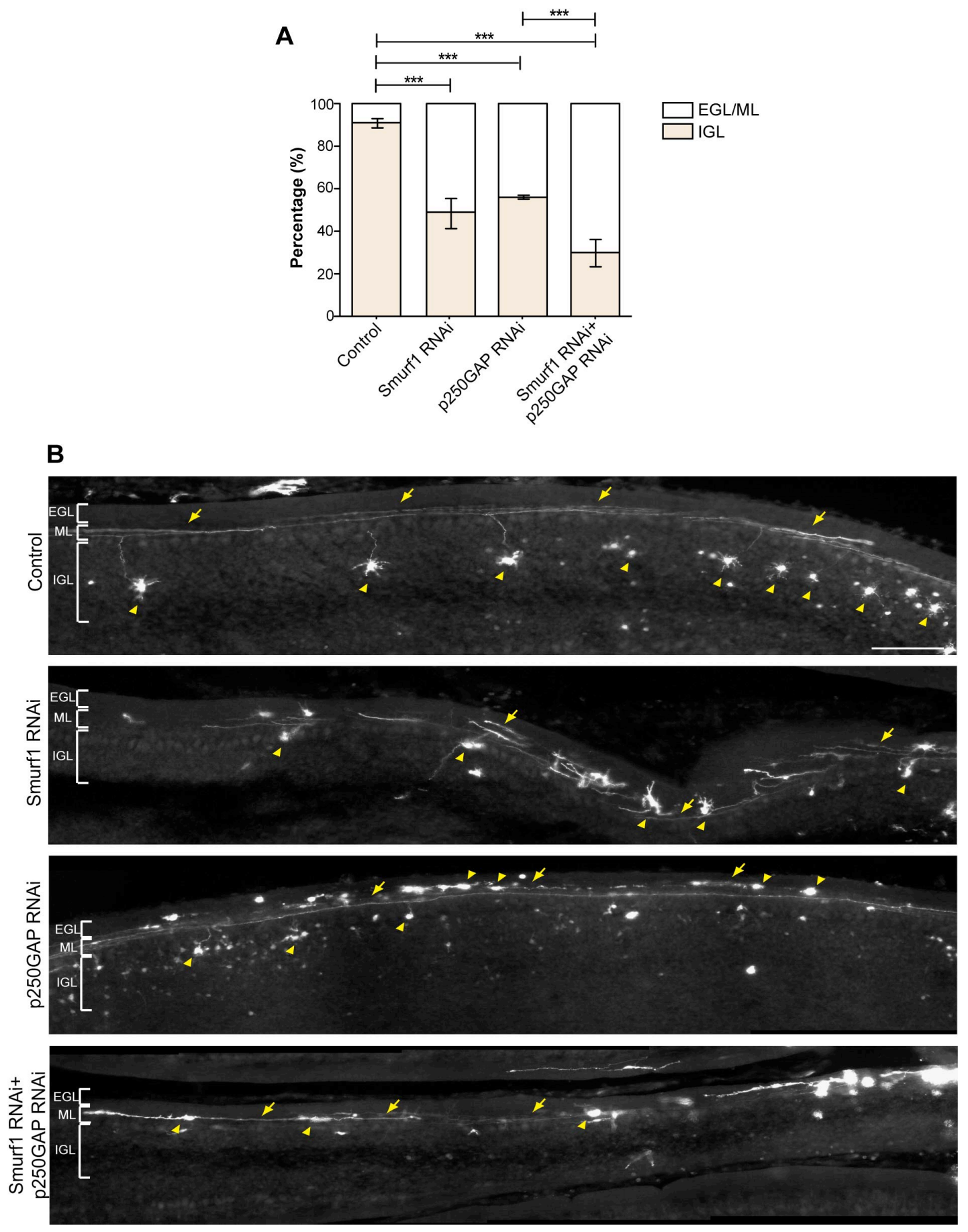

Figure 3.43 Smurf1 and p250GAP are essential for cerebellar granule neuron migration in vivo. In vivo electroporation of rat cerebella was performed in collaboration with Dr. Judith Stegmüller and Shih-Ju Lee, MPIEM, Göttingen. While I isolated and screened GFP-positive cerebella, fixed and embedded tissue, S.J. Lee performed cryo-sections, immunohistochemistry and analyzed the individual sections together with Dr. Stegmüller. A. The control U6-CMV-EGFP plasmid, p250GAP RNAi-CMV-EGFP (p250GAP RNAi), Smurf1 RNAi-CMV-EGFP (Smurf1 RNAi), or both p250GAP RNAi and Smurf1 RNAi bi-cistronic plasmids together with $\mathrm{BCL}_{\mathrm{XL}}$ plasmid, were injected into the cerebellum of $\mathrm{P} 4$ rat pups. At P9, cerebella were isolated and coronal sections were subjected to immunohistochemistry using the GFP antibody. The location of transfected neurons was assessed as either external granule layer (EGL)/molecular layer (ML) or internal granule layer (IGL). A total of 10527 neurons was counted (ANOVA, $* * * p<0.0001$, values indicates mean $+\mathrm{SEM}$ ). B. Representative images of each condition. Scale bar equals $100 \mu \mathrm{m}$. Arrows indicate parallel fibers and processes and arrow-heads indicate cell bodies. 
Analysis of GFP-positive cerebella of control versus knockdown injections revealed a striking phenotype in the knockdown conditions. In control cerebella, granule neurons exhibit a developmental profile typical of P9. They migrate inward from the cerebellar cortex, which consists of a transient external granule layer (EGL), and descend into the internal granule layer (IGL) across the intermediate molecular layer (ML). In doing so, granule neurons form bifurcated axons that extend in the ML and fasciculate into parallel fiber bundles to span the entire length of the cerebellum. The granule neurons eventually develop a dendritic arbor when they reach their final destination (IGL) (Schematic 3C).

In contrast, we discovered that under p250GAP or Smurf1 knockdown, the neurons display an aberrant phenotype. Here, more granule neurons are stalled in the EGL/ML. While $90 \%$ of the control neurons migrate to the IGL, only $56 \%$ and $49 \%$ of p 250 GAP and Smurf1 knockdown neurons descend, respectively. The migration defect is further exacerbated in the double knockdown condition, in which barely 30\% neurons reach the IGL (Figure 3.43). This is possibly the outcome of additive effects of p250GAP and Smurf1-regulated migration. Our analyses indicate that both $\mathrm{p} 250 \mathrm{GAP}$ and Smurf1 are crucial for the proper migration of cerebellar granule neurons in vivo.

\subsection{2 p250GAP or Smurf1 knockdown distorts the development and elongation of} granule neuron parallel fibers in the early postnatal rat cerebellum

In our in vivo analyses, we found additional morphological defects in the knockdown neurons. The stalled neurons in the knockdown conditions extend shorter and rather thick axons that fail to develop into characteristic parallel fibers. The quantification of short and thick versus long and thin processes revealed a stark phenotype in the knockdown neurons (Figure 3.44A, C).

Further, we also observed a glaring difference in the axonal lengths between control and knockdown neurons. We had to develop an arbitrary technique to quantify this difference, since it is impossible to measure the length of the parallel fibers of the control neurons. We measured the length of the parallel fibers as long as we could trace them in a section and thus determined the minimal length of these processes. For knockdown neurons, however, it was still feasible to measure the entire axon length as we could determine the tips of short grown axons. Our assays revealed that control axons extend for at least $390 \mu \mathrm{m}$, while p250GAP or Smurfl or the double knockdown neurons have an average axon length of $150 \mu \mathrm{m}$ (Figure 3.44B, C). While p250GAP and Smurf1 appear to act in independent pathways in granule 
neuron migration, the two proteins act together in the control of axon growth in vitro (Figure 3.44) and in vivo suggesting common downstream cascades that may involve the small GTPase RhoA.
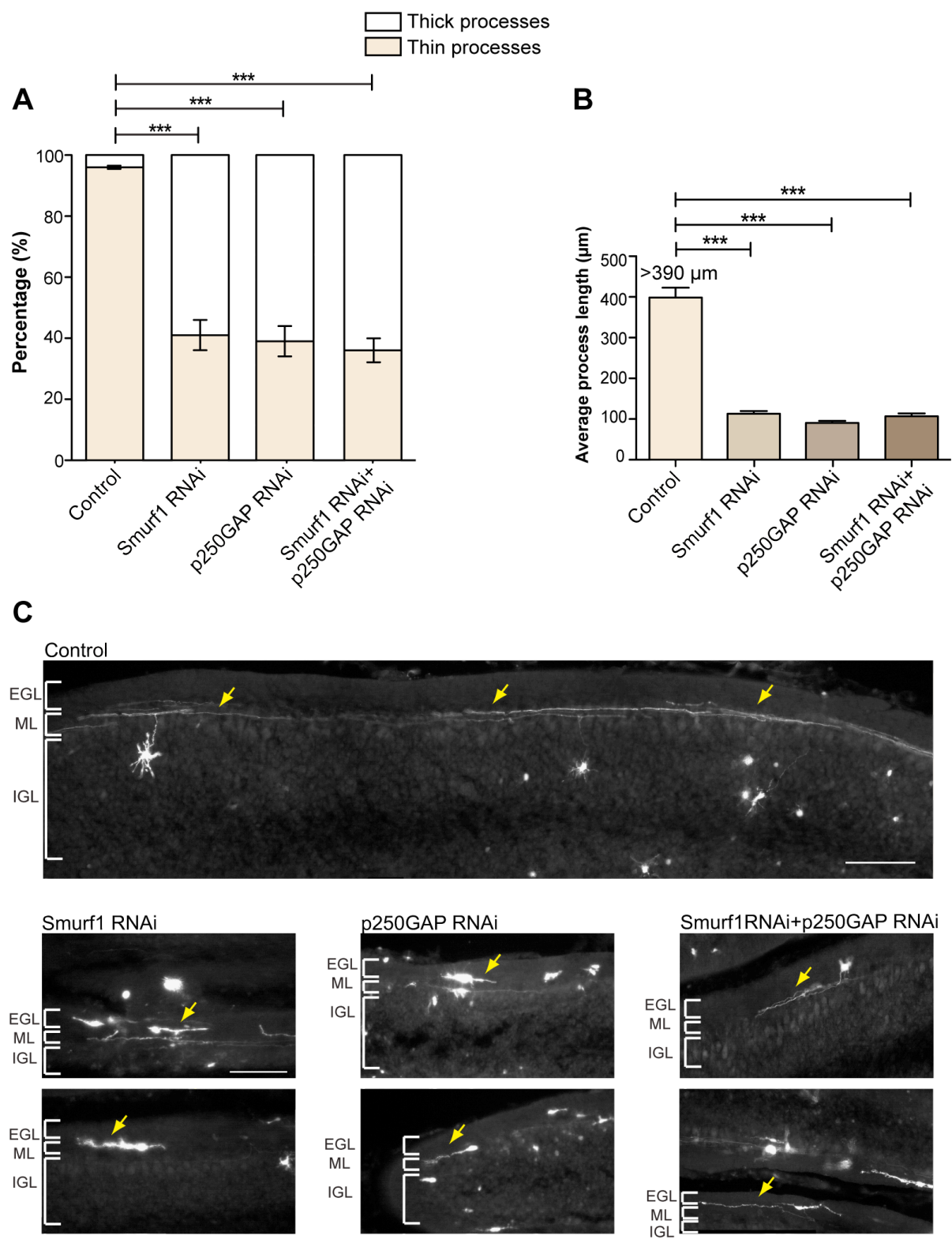

Figure 3.44 Smurf1 and p250GAP regulate granule neuron axonal morphogenesis in vivo. In vivo electroporation of rat cerebella was performed in collaboration with Dr. Judith Stegmüller and Shih-Ju Lee as described in 3.45. A. Quantification of thin (normal) processes and short, thick processes. A total of 1200 processes was counted (ANOVA, ${ }^{* * *} \mathrm{p}<0.0001$, values indicate mean $+\mathrm{SEM}$ ). B. Quantification of axonal length. A total of 325 neurons was measured (ANOVA, $* * * p<0.0001$, values indicate mean + SEM). C. Representative images of each condition in $\mathbf{A}$ and $\mathbf{B}$. EGL=external granule layer, $\mathrm{ML}=$ molecular layer, $\mathrm{IGL}=$ internal granule layer. Scale bar equals $100 \mu \mathrm{m}$. Arrows indicate parallel fibers and processes.

Collectively, our in vivo analyses reiterate a role for p250GAP and Smurf1 in axon extension in the developing cerebellar cortex and uncover a novel role for the two proteins in regulating cerebellar granule neuron migration. 


\section{Discussion}

Neuronal development is tightly regulated by a plethora of extrinsic and intrinsic molecular events. The extrinsic factors evoke signaling cascades to permit or retard axon growth by inducing neuronal transcriptional activity or by modulating the activity states of cellular proteins. The effects of these are in turn, spatially and temporally fine-tuned by an intricately coordinated protein turnover. Thus, by controlling the turnover of axon growth modulators, the cell-intrinsic ubiquitin proteasome system (UPS) contributes to a multi-level regulation of neuronal development.

Cdh1-APC is a multi-subunit E3 ligase discovered as an axon growth inhibitor in the mammalian brain (Konishi et al., 2004). Though studies have identified downstream pathways in the cell-intrinsic role of Cdh1-APC in axon growth (Lasorella et al., 2006; Stegmuller et al., 2006), what remains unknown is how the complex integrates signals from extrinsic factors viz. neurotrophins, in its function. My study elaborates the cell-autonomous role of the E3 ligase Cdh1-APC in axon growth regulation and attempts to link extrinsic players and their intracellular modulators to the process.

The flip side of axon growth is axon regeneration. In contrast to the adult PNS, the CNS is limited in its regenerative capacity. Not only are injured CNS neurons more prone to extrinsic inhibition by myelin and glial scar but they are also incapable of triggering transcriptional programs for repair (Liu et al., 2011; Vargas and Barres, 2007; Yiu and He, 2006). Intrinsic inhibitors of axon regeneration are invariably inhibitors of axonal growth during development (Lasorella et al., 2006; Moore et al., 2009; Park et al., 2008; Yu et al., 2011). Thus, by resolving neurodevelopmental processes, it is likely that we can break the fetters in neuronal repair. Thus, my study offers an insight not only into Cdh1-APC pathway of axon growth inhibition but also underscores its potential role in adult regeneration.

\subsection{MAPK and PI3K signaling antagonize Cdh1-inhibition of axon growth}

The mitogen activated protein kinase (MAPK) pathway integrates signals from neurotrophins, particularly nerve growth factor (NGF) and brain-derived neurotrophic factor (BDNF), to induce cAMP response element binding protein (CREB)-dependent transcriptional activity in promoting neurite growth (Lonze and Ginty, 2002). Pharmacological inhibition of 
MAPK signaling reduces axon growth in Cdh1 knockdown neurons in vitro. Moreover, analyses of cerebella from Cdh1 heterozygotes show evidence of induced MAPK signaling.

The phosphotidylinositol 3-kinase (PI3K) pathway on the other hand, affects both transcriptional activity and cytoskeletal proteins in response to neurotrophins to promote neurite growth (Goold and Gordon-Weeks, 2004). My experiments indicate a putative role for PI3K in Cdh1-APC control of axon growth though the relevant mechanism is still unclear.

The results suggest that Cdh1-APC may act as an intrinsic brake to counteract extrinsic factors (such as neurotrophins) that elicit intracellular cascades in promoting axon growth. Despite being in an axon growth-stimulating environment rich in trophic factors, the existence of intrinsic inhibitory proteins may ensure that a fully differentiated/mature axon does not overshoot its synaptic targets. It is unknown, however, whether components of MAPK or PI3K pathways are substrates of Cdh1-APC. Conversely, a MAPK or PI3K-regulation of Cdh1 activity is also plausible owing to the reported phosphorylation-dependent regulation of Cdh1 activity (Huynh et al., 2009).

\subsection{Cdh1-APC controls axon growth by modulating cytoskeletal proteins}

Extrinsic factors not only induce neuronal transcriptional activity but also modulate the activity states of cytoskeletal proteins to permit cytoskeletal events in axon growth and extension. Cdh1-APC inhibits axon growth in the mammalian brain (Konishi et al., 2004), with the transcription factors SnoN and Id2 described as its two prominent substrates in this respect (Lasorella et al., 2006; Stegmuller et al., 2006). However, it is unclear how Cdh1-APC may control the activity of cytoskeletal proteins particularly components regulating actin and microtubule dynamics, given that the latter are the major contributors to axon initiation and extension.

Rho GTPases are major effectors of extrinsic signaling (Dickson, 2001; Govek et al., 2005). The small GTPases have an unsurpassing role in the regulation of cytoskeletal dynamics and have been extensively described in neurite initiation and outgrowth, axon extension, axon pathfinding, dendritic formation and axon regeneration (Govek et al., 2005). Thus, I examined whether Cdh1-APC exerts its role in axon growth by modulating the classical Rho GTPases RhoA, Rac1 or Cdc42.

Early studies that deciphered the role of Rho GTPases in neurite initiation and outgrowth were performed in PC12 cells. Local activation of Rac1 and Cdc42 at neurite tips 
induce lamellipodia and filopodia formation while dominant negative Rac1 (Rac1 DN) or Cdc42 DN inhibit neurite outgrowth (Aoki et al., 2004). RhoA activation, on the contrary, is associated with neurite retraction while RhoA DN produces the protrusive structures characteristic of Rac1 or Cdc42 activation (Govek et al., 2005). In a very broad sense Rac1 and $\mathrm{Cdc} 42$ are described as 'positive' regulators while RhoA is a 'negative' regulator of neurite outgrowth. This is also true of their respective roles in axon initiation and extension. However, there are many exceptions to this rule (Govek et al., 2005).

The finding that RhoA overexpression overcomes Cdh1 knockdown-induced axon growth in granule neurons suggests that RhoA GTPase may mediate Cdh1-APC-dependent axon growth inhibition. Further, the lack of additive effects of RhoA and Cdh1 is apparent and the RhoA phenotype is dominant in the background of Cdh1 knockdown. RhoA WT reduces axon length in Cdh1 knockdown neurons but on its own, has no effect on axon growth; similarly, RhoA DN has a profound growth promoting effect on its own and no additive effect when expressed together with Cdh1 RNAi.

Unlike RhoA, coexpression of any of Rac1 WT, Rac1 CA or Rac1 DN in Cdh1 RNAi neurons results in a significant reduction in axon length. Though Rac1 GTPase largely promotes axon extension, contrasting effects have also been observed. Rac1 CA expressing transgenic mice show reduced Purkinje cell axon terminals (Luo et al., 1996). Rac1 DN on the contrary, enhances axon growth in certain systems, viz. Drosophila motor neurons (Kim et al., 2003). Thus, critical levels of Rac1 activity may be required to regulate axon growth and the system falters whenever Rac1 activity is manipulated. Besides, a common explanation to CA and DN mutants giving rise to similar phenotypes is that Rho GTPase signaling has a cyclic mode of action. If protrusive structures cycle between extension and retraction in growth cone motility, then blocking this dynamic process either with a CA or DN mutant will have the same net effect of inhibited axon growth (Luo, 2000).

While neither Cdc42 WT nor DN has any effect, CA inhibits the Cdh1 RNAi-long axon phenotype. Cdc42 WT may be prone to regulation by GAPs, which are enriched in the developing brain (Moon and Zheng, 2003; Tcherkezian and Lamarche-Vane, 2007) (inhibiting its activity) and this may explain why WT does not exhibit an inhibitory effect while a CA mutant abrogates axon growth in these neurons. Again, a strongly growth-inhibiting effect of Cdc42 CA has been noted before in neuritogenesis (Allen et al., 2000). Also, though Cdc42 is extensively characterized in regulating neuronal polarity, its role in the regulation of axon growth is relatively unexplored. 


\subsection{E3 ligase Smurf1: a novel Cdh1 substrate that potentiates axon growth}

Aside from inhibiting axon growth in development, Cdh1-APC has also been implicated in myelin inhibition of axon growth (Konishi et al., 2004). Myelin is the notorious cause for the lack of adult regenerative capacity in the brain (McKerracher et al., 1994; Mukhopadhyay et al., 1994; Schwab, 1993). Myelin proteins exert their inhibitory effect by binding to Nogo receptor and the $75^{\mathrm{NTR}}$ co-receptor complex (Wang et al., 2002). The latter displaces inactive RhoA from RhoA GDI facilitating its activation by GEFs (Yamashita and Tohyama, 2003). Thus, the inhibitory effect of myelin is relayed forward by RhoA activation and RhoA is unanimously the crucial downstream component of myelin signaling. These observations taken together with my finding that RhoA overcomes Cdh1 RNAi-induced axon growth, prompted me to further dissect the molecular mechanism of Cdh1-APC/RhoA crosstalk.

I reasoned that RhoA being growth inhibitory itself is unlikely to be a direct substrate of the growth inhibiting Cdh1-APC, and hence postulated that proteins negatively regulating RhoA/RhoA activity may sandwich the Cdh1-APC-to-RhoA-gap. Interestingly, RhoA levels are regulated by proteasomal turnover. Smurf1, a homologous to E6AP C-terminus (HECT) ubiquitin ligase has been described in great detail as a negative regulator of RhoA levels (Boyer et al., 2006; Sahai et al., 2007; Wang et al., 2003).

Smurf1 belongs to the family of HECT ubiquitin ligases that actively participates in substrate ubiquitination by forming a ubiquitin-conjugated intermediate. Like all HECT ligases, Smurf1 harbors a catalytic cysteine residue in its C-terminal HECT domain that binds the activated ubiquitin before it is transferred to the substrate.

Orginally discovered for its role in bone morphogenetic protein (BMP) signaling as a negative regulator of Smads 1 and 5 in embryonic pattern formation (Zhu et al., 1999) and later in JNK signaling upstream of MEKK2 in osteoblast differentiation (Yamashita et al., 2005), Smurf1 has also been described as a RhoA antagonist (Boyer et al., 2006; Sahai et al., 2007; Wang et al., 2003). In the latter respect, Smurf1 has been reported as a neurite growth promoting protein (Bryan et al., 2005; Sato and Heuckeroth, 2008; Vohra et al., 2007). In a contemporaneous paper, Smurfl's role upstream of RhoA in axon extension in CNS neurons was uncovered (Cheng et al., 2011). Even as Smurfl was identified in the lab as a novel interactor of Cdh1, a parallel study published the interaction in the context of osteoblast differentiation (Wan et al., 2011). 
Smurf1 but not its homolog Smurf2 specifically interacts with Cdh1 in vitro and in vivo. Initial characterization revealed that Smurf1 is present both in the nucleus and cytoplasm in neurons, a feature that supports its involvement in shuttling Smad7 between nucleus and cytoplasm in transforming growth factor $\beta$ (TGF- $\beta$ ) receptor turnover (Ebisawa et al., 2001). Axon assays confirmed the axon growth-promoting role of Smurf1 in cerebellar granule neurons and cortical neurons emphasizing a generalizable growth-regulating effect of the HECT ligase.

I further identified that the axon growth-promoting role of Smurf1 depends on both its nuclear and cytoplasmic localization since expression of both NLS- and NES-Smurfl only partially rescues the Smurfl knockdown-induced short axon phenotype. These observations have the following interpretations: (i) Smurf1 NLS may be spatially incompetent in degrading RhoA and hence only partially effective in axon growth regulation, (ii) NES-Smurfl's inability to completely rescue Smurfl knockdown indicates as yet elusive mechanisms of Smurf1 regulation and/or unidentified Smurf1 targets in the nucleus are key to its role in axon growth induction, and (iii) a Smurf1 NES mutant (Smurf1 NLS) fails to promote Smad7-dependent inhibition of TGF-ß-transcriptional activity (Tajima et al., 2003). The latter has been implicated in concert with Cdh1-APC in axon growth inhibition (Stegmuller et al., 2008).

In epistasis analysis, I found that Smurfl is a downstream component of the Cdh1-APC pathway. This finding prompted me to speculate that Smurf1 is a potential Cdh1 substrate. The following biochemical observations further bolstered my hypothesis. Smurf1 is regulated by proteasomal turnover in heterologous cells (Cui et al., 2011). Smurf1 protein expression is induced upon proteasomal inhibition in granule neurons suggesting similar UPS modulation of Smurf1 levels in neurons. The key finding that Smurfl protein but not mRNA is up-regulated in the Cdh1 heterozygotes indicates that Smurf1 stability depends on Cdh1 levels. In addition, Smurf1 ubiquitination is slightly but consistently reduced in brain lysates from Cdh1 heterozygotes lending support to a Cdh1-mediated proteasomal turnover of Smurf1 in the mammalian brain.

A recent report indicated a Cdh1-dependent Smurf1 activation and autoubiquitination in osteoblasts (Wan et al., 2011). The authors proposed a mechanism whereby Cdh1 independent of its own E3 ligase activity, enhances the instrinsic ligase activity of Smurf1 by disrupting an autoinhibitory homodimer. Under Cdh1 knockdown however, Smurfl is inactivated and downstream cascades viz. those involving MEKK2 that are normally inhibited by Smurf1, are induced to facilitate osteoblast differentiation. While the paper proposes a 
'ligase-independent' role for Cdh1 in Smurf1 turnover, the ubiquitination assays in heterologous cells, presented in the Results section, identify a Cdh1 'ligase-dependent' Smurf1 ubiquitination. Smurf1 ubiquitination is induced by Cdh1 WT but not by Cdh1 9D, a catalytically inactive mutant that is dissociated from the APC core. Moreover, previous findings that overexpression of wild-type or a constitutively active Cdh1 has no gain-offunction in axon growth suggest that the axon growth modulatory role of Cdh1 is dependent on its association with the APC core (Judith Stegmueller, Azad Bonni, Department of Pathology, Harvard Medical School, unpublished results). Taken together, my results reveal an E3 ligasedependent role for Cdh1 in regulating Smurf1 levels in the developing nervous system. The existence of different mechanisms of Smurf1 regulation by Cdh1 in different cell types may ensure different roles for Smurfl in the bone and neuronal tissues. Also, differences in the availability of other components of the Cdh1-APC machinery including the APC core itself for functions besides cell cycle regulation in the mitotic osteoblasts versus postmitotic neurons may account for such varied mechanisms of Cdh1-Smurf1 interaction in the two cell types.

Cdh1 recognizes signature sequences in substrates (Glotzer et al., 1991; Littlepage and Ruderman, 2002; Pfleger and Kirschner, 2000), mutations of which enhance substrate stability (Lasorella et al., 2006; Stegmuller et al., 2006; Stroschein et al., 2001). Notably, the D-box mutants (DBMs) of SnoN and Id2, Cdh1 targets in axon growth regulation, are remarkably stabilized and exhibit a gain-of-function in axon growth (Lasorella et al., 2006; Stegmuller et al., 2006). Similarly, Smurf1 D-boxes 3 and 4 mediate its interaction with Cdh1 and hence, are crucial for promoting axon growth downstream of Cdh1-APC. Interestingly, D-box 3 is at position 358 and lysines K355 and K357 in close proximity (see Schematic 3A in Results) have been identified as the key lysine residues involved in Smurf1 ubiquitination by another Smurf1 regulator FBXL15 (Cui et al., 2011). It may be worth to validate the role of these residues in Cdh1-mediated Smurf1 ubiquitination.

Does the Cdh1/Smurf1 pathway involve one or both of the known axon growthregulating Cdh1 targets, SnoN and Id2? Experiments involving the simultaneous expression of Smurf1 RNAi and SnoN DBM or Id2 DBM, the growth-promoting gain-of-function mutants of SnoN and Id2 respectively, showed an absence of epistasis of either condition. Thus, Smurf1 downstream of Cdh1-APC controls axon growth independent of SnoN or Id2. Overall, my findings implicate several independent targets in the Cdh1-APC pathway of axon growth control emphasizing the importance of UPS in a multimodal regulation of neuronal development. The phenomenon is reminiscent of the role of Cdh1-APC in recruiting multiple 
substrates viz. Cdc20, Cylin B1, Securin and Aurora for proteasomal degradation in cell cycle control (Li and Zhang, 2009).

\subsection{Smurf1-degradation of RhoA is central to Cdh1-APC/Smurf1 pathway}

Further, Smurf1 operates downstream of Cdh1 in the Cdh1-APC/RhoA axis as a regulator of RhoA. Wang et al first reported a Smurf1-dependent ubiquitination and degradation of RhoA (Wang et al., 2003). They discovered that Smurfl as an effector of Cdc42/Rac1-Par6-PKC $\zeta$ pathway, degrades RhoA at the leading edge of protrusions in tumor cells. RhoA degradation by Smurf1 soon came to be known as a pivotal mechanism not only in epithelial-to-mesenchymal transition (EMT) in metastasis (Sahai et al., 2007) but also in regulating neurite growth in neuroblastoma cells (Bryan et al., 2005), axon elongation in enteric neurons (Vohra et al., 2007) and axon development in the CNS (Cheng et al., 2011). Concurrent with these findings, a degradation-resistant RhoA mutant annuls axon growth stimulated either by Cdh1 RNAi or Smurf1 DBM3/4. Thus, Smurf1-degradation of RhoA is central to the Cdh1-APC/RhoA pathway of axon growth control. Taken together, an E3 ligase cascade of Cdh1-APC and Smurf1 exerts control of axon growth upstream of the small GTPase RhoA.

One would expect that, as a component of the Cdh1-APC pathway, RhoA levels are similarly affected (reduced) as are Smurf1 in the Cdh1 heterozygotes. However, neither total nor active RhoA levels are affected in the $\mathrm{Cdh}^{+/-}$mice. Indeed, RhoA is a downstream component of several cascades involved in a plethora of neuronal processes viz. neuritogenesis (Mackay et al., 1995), axonal and dendritic morphogenesis (Dickson, 2001; Govek et al., 2005; Luo, 2000), neuronal migration (Govek et al., 2011), and stress fiber formation (Kranenburg et al., 1997) and hence, no single pathway may entirely abrogate its global levels. Moreover, changes in RhoA activity in neurite retraction are specifically detected at the cell periphery (Yamaguchi et al., 2001). The study by Cheng and colleagues also points to a preferential localization of active Smurfl in the tips of axons in RhoA turnover (Cheng et al., 2011). Concordant with these observations, hippocampal neurons transfected with Cdh1 RNAi show reduced RhoA staining at axon tips. This may reflect a local increase in Smurf1 levels.

Huynh and colleagues in 2009 reported a predominant catalytic role for Cdh1 in the nucleus in the control of axon growth (Huynh et al., 2009). How then does Cdh1-APC affect local RhoA levels at axon tips? The apparent paradox can be explained by a localized activity of Cdh1-APC in the cytoplasm. Indeed, only small amounts of Cdh1 are detected in the 
cytoplasm (Konishi et al., 2004) suggesting that APC-associated Cdh1 activity may be very low outside the nucleus and localized Cdh1-APC activity may cause local changes in RhoA levels at axon tips. Moreover, Smurf1 levels are well up-regulated in the Cdh1 heterozygotes, a single allele loss does not produce a pronounced effect on global RhoA levels, RhoA being further downstream in the pathway.

The identification of extranuclear substrates of Cdh1-APC in regulating other aspects of neuronal development supports the existence of an active Cdh1-APC complex in the cytoplasm. In response to ephrin A signaling, Cdh1-APC controls the abundance of AMPA receptor, GluR1 in hippocampal synaptic plasticity (Fu et al., 2011). The identification of Dboxes that facilitate Cdh1-GluR1 interaction and ubiquitination of the receptor by wild-type but not a dominant negative Cdh1 confirm a direct role for Cdh1-APC in regulating GluR1 levels in dendritic spines (Fu et al., 2011). Reduced late-long term potentiation (L-LTP) in hippocampal slices from $\mathrm{Cdh} 1$ heterozygotes further underscores the role of Cdh1 in regulating unknown substrates at synapses ( $\mathrm{Li}$ et al., 2008). Unlike mammalian systems, Cdh1 fusion proteins in Drosophila predominantly localize to the cytoplasm and axons in postmitotic neurons (Silies and Klambt, 2010). In the fly, synaptic proteins GLR-1 and liprin- $\alpha$ are both regulated by Cdh1-APC activity in the control of synapse size and activity (Juo and Kaplan, 2004; van Roessel et al., 2004). The detection of endogenous Cdh1 in different cellular compartments is obscured due to a lack of good antibodies for immunostaining despite efforts of several labs including ours. Discrepancies in subcellular localization of Cdh1 hence arise from the relative differences in protein distribution between various tissues and across species.

My finding reiterates a role for Smurf1/RhoA pathway in axon growth with Cdh1-APC as an additional member upstream. In a broader context, my work adds to the repertoire of E3 ligase-regulated small GTPases in neuronal development. Besides Smurf1/RhoA discussed above, Smurf2, another E3 ligase has been shown to degrade Rap1B GTPase, an essential downstream player of the PI3K/Akt pathway in the establishment of hippocampal neuron polarity (Schwamborn et al., 2007). Another recent report has ascribed a role for Nedd4, a HECT ubiquitin ligase in the inactivation of Rap2A GTPase in facilitating dendritic extension and arborization (Kawabe and Brose, 2011).

\subsection{Smurf1 promotes neuronal migration and axonal growth in vivo}

Granule neuron migration in cerebellar development is illustrated in Schematic $3 \mathrm{C}$ in Results. 
In vivo knockdown of Smurf1 results in a prominent deficit of granule neuron migration. In addition, these neurons also display conspicuous defects in axonal morphology. In contrast to thin and long processes that develop into characteristic parallel fibers in the control condition, the Smurf1-RNAi neurons possess very short and thick processes and have no parallel fibers.

Our in vivo results are consistent with the study by Cheng and colleagues that reported aberrant migration of Smurfl knockdown neurons in cortical development (Cheng et al., 2011). They observed a failure of radial migration of cortical neurons upon Smurfl knockdown in addition to polarity defects in these neurons. These findings taken together with my in vitro and in vivo assays, establish a more generalized role for Smurfl in axon growth and neuronal migration in the developing mammalian brain.

The data on Smurfl's role in cerebellar granule neuron migration open portals to a likely involvement of RhoA in the process. RhoA GTPase has been extensively studied in cortical neuron migration. In the developing cortex, RhoA expression is confined to ventricular and subventricular zones (VZ/SVZ). It is however, sharply down-regulated during cortical neuron migration (in the intermediate zone or IZ) as a result of tight regulation by upstream proteins including the proneural bHLH factor, neurogenin-2 and the cyclin-dependent kinase inhibitor, p27 ${ }^{\text {Kipl }}$ (Ge et al., 2006; Nguyen et al., 2006). Expression of RhoA DN has been shown to rescue migratory defects in Ngn-2 null embryos (Hand et al., 2005) and restore migration to the cortical plate (CP) under p2 ${ }^{\text {Kip1 }}$ knockdown (Nguyen et al., 2006). These studies establish RhoA as a negative regulator of radial migration in cortical development. A similar check on RhoA levels or activity may be required to drive cerebellar granule neuron migration, suggestive of a role for Smurf1 upstream.

\subsection{Smurf1 is a candidate in regenerative therapy}

CNS axon regeneration is very limited owing to extrinsic inhibition as well as an intrinsic lack of growth capacity in mature neurons (Yang and Yang, 2012; Yiu and He, 2006). Myelin proteins are renowned extracellular inhibitors of axon regeneration whose effects converge on the downstream player, RhoA. A few studies have shown that such extrinsic suppression can be overcome by relieving intrinsic inhibition (Konishi et al., 2004; Moore et al., 2009; Park et al., 2008). For example, Cdh1 knockdown overrides myelin inhibition of axon growth (Konishi et al., 2004). Smurf1 DBM3/4, that is resistant to Cdh1-degradation, similarly enhances axon growth on myelin. Thus, by overcoming intrinsic growth incapacity, a 
stabilized Smurfl potentiates axon growth on non-permissive substrates, possibly by regulating RhoA.

A recent report identified Id2 DBM in alleviating spinal cord injury (Yu et al., 2011). Dorsal root ganglion (DRG) neurons transfected with Id2 DBM produce longer axons that fill the lesion compared to control neurons following dorsal funiculotomy. In addition, Id2 DBM greatly reduces axonal dieback after lesion, an early event after axon severing (Yu et al., 2011). Id2 DBM may overcome the Cdh1-APC-dependent intrinsic inhibition (Lasorella et al., 2006) to facilitate axon recovery. Smurfl DBM, that overrules myelin inhibition of axon growth in vitro, may similarly represent a promising candidate for therapeutic intervention.

\subsection{The Cdh1-APC/Smurf1/RhoA pathway: scope for further research}

To sum up, my study identifies Smurf1 as a novel substrate of Cdh1-APC upstream of RhoA in axon growth control (Schematic 4A). Future studies will require a more comprehensive analysis of the Smurf1 knockout and the generation of a conditional mutant. The Smurfl conventional knockout does not show any overt phenotype (Yamashita et al., 2005). However, the analysis has been rather restricted to bone formation, and points to an induced MEKK2-mediated osteoblast formation in the $\mathrm{Smurfl}^{-/-}$mice (Yamashita et al., 2005). The report also indicates increased Smurf2 transcript levels in these mice and attempts to explain this as a compensatory mechanism. Recently, Narimatsu and colleagues characterized Smurf1/2 double knockout mice, which die around embryonic day 10 (E10) (Narimatsu et al., 2009). The group discovered neural tube closure defects, as well as impaired cochlear planar cell polarity (PCP) signaling in the Smurf1/2/- embryos, the individual contribution of the Smurfs to these deformities is however, unclear. Rescue experiments with Smurfl overexpression in Smurf2 ${ }^{-/}$and Smurf2 overexpression in $\mathrm{Smurf1}^{-/-}$mice may be more informative. A redundancy of the two Smurfs may not be entirely fitting in all tissues considering that the two HECT ligases have varied roles in the developing brain (Bryan et al., 2005; Cheng et al., 2011; Schwamborn et al., 2007; Vohra et al., 2007) and in at least one instance, they may have contrasting roles (Fukunaga et al., 2008).

An inducible mutant of Smurf1 will be a valuable tool to further exploit the role of Smurf1 and RhoA in CNS axon growth and regeneration. It may be convenient to study local RhoA levels in developing axons in these animals and in the event of induced RhoA, to perform rescue assays with RhoA DN or RhoA/Rho-kinase (ROCK) inhibitors. Additionally, manipulation of RhoA activity in myelinated tissues after nerve injury (viz. spinal cord injury 
or optic nerve crush) may offer an insight into the potential role of Smurfl/RhoA in axon regeneration.

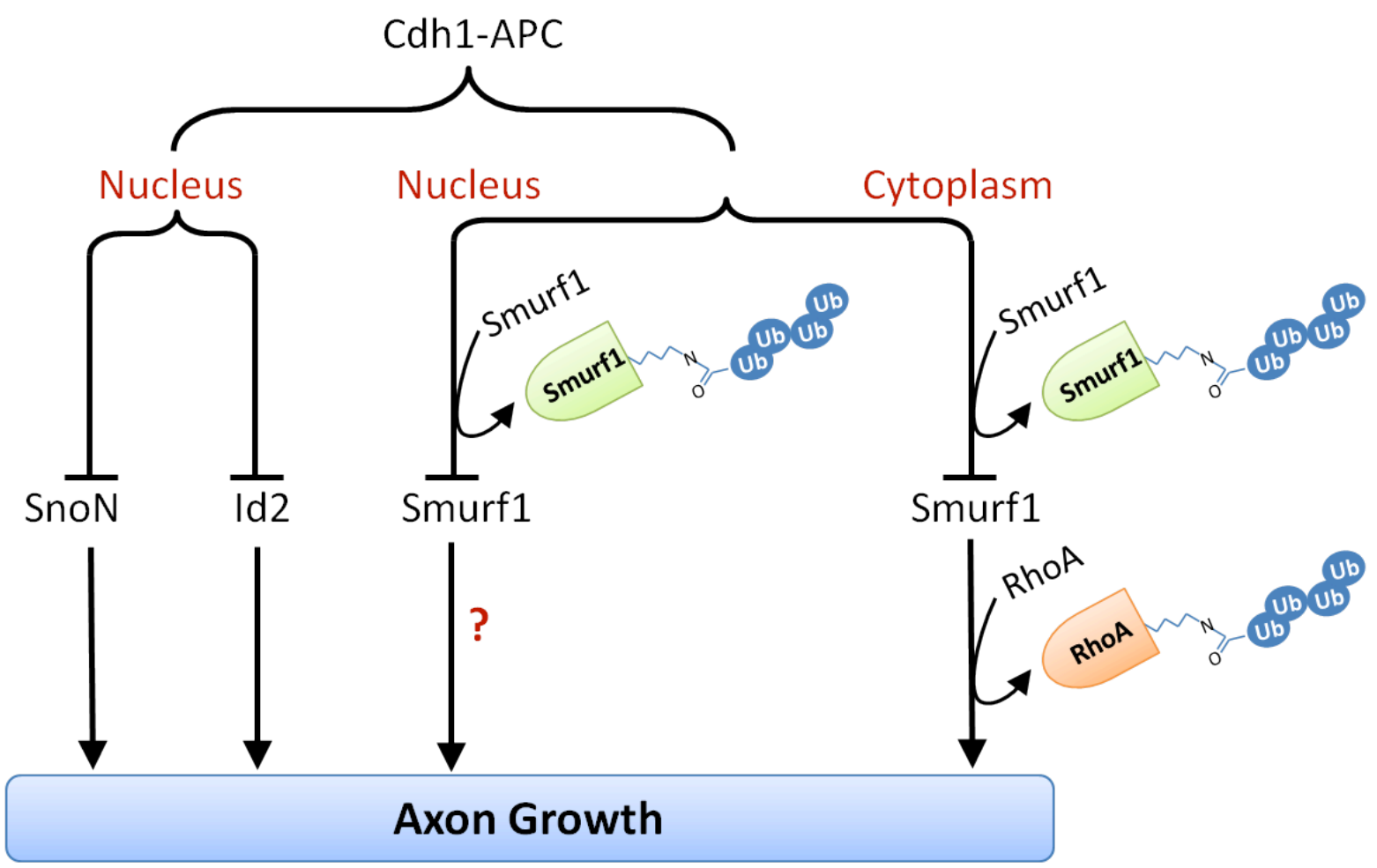

Schematic 4A. The Cdh1-APC/Smurf1/RhoA pathway of axon growth regulation. The E3 ligase Cdh1-APC targets Smurf1 for proteasomal degradation and thus, contributes to axon growth inhibition in a RhoA-dependent manner. While RhoA is the cytoplasmic target of Smurf1 in axon growth control, Smurf1's nuclear substrates are unknown. The Cdh1-APC/Smurf1 pathway is independent of the two known Cdh1 targets, SnoN and Id2. The existence of several parallel pathways of Cdh1-regulation is suggestive of a multimodal regulation of axon growth by the E3 ligase.

In addition to regulating RhoA, the role of Smurf1 in controlling neuronal morphogenesis may extrapolate along other pathways. By destabilizing hPEM-2, a Cdc42 GEF, Smurf1 may also exert a negative influence on the activity of Cdc42 (Yamaguchi et al., 2008), another positive regulator of Cdh1-mediated axon growth inhibition. Also, our in vitro assays point to a potential role of Smurf1 in dendritic development (Shih-Ju Lee and Judith Stegmüller, unpublished results). This raises the possibility of a cross-talk between Smurf1 and Cdc20, the mitotic activator of the APC complex. The E3 ligase, Cdc20-APC promotes dendritic growth in postmitotic neurons (Kim et al., 2009). Given that Cdc20 recognizes similar degrons as Cdh1 (Glotzer et al., 1991), Smurf1 is a candidate interactor of Cdc20-APC. Finally, it will be of therapeutic value to identify the extracellular molecular events upstream of Cdh1-APC/Smurf1/RhoA for cues to circumvent myelin inhibition of axon growth after injury and repair. 


\subsection{GAPs are candidate mediators of $\mathrm{Cdh} 1$-dependent RhoA regulation}

As mentioned above, in the Cdh1-APC/RhoA pathway of axon growth regulation, I postulated that proteins negatively regulating RhoA/RhoA activity may sandwich the Cdh1APC-to-RhoA-gap.

Besides Smurf1, RhoA is subject to extensive regulation by guanine nucleotide exchange factors (GEFs), GTPase activating proteins (GAPs) and guanine nucleotide exchange inhibitors (GDIs). The RhoGAPs inactivate Rho GTPases by enhancing their intrinsic GTPhydrolyzing activity. I speculated that a RhoA GAP may be a potential Cdh1-APC substrate and operate upstream of RhoA in the Cdh1-APC/RhoA pathway of axon growth regulation.

RhoGAPs have been assigned important roles in neuronal function. The prototypical p190GAP is enriched in the mouse brain and in fact, the most abundantly phosphorylated protein in whole brain lysates. p190GAP knockouts display stark axon guidance defects in the brain and axon fasciculation defects in cranial nerves and die perinatally (Brouns et al., 2001). p190GAP enhances dendritic spine density in hippocampal neurons downstream of the polarity protein Par6, by inactivating RhoA (Zhang and Macara, 2008). Additionally, p190GAP as a RhoA inactivator, has been implicated in long-term memory formation in fear conditioning (Lamprecht et al., 2002). Another RhoGAP, oligophrenin-1 (Ophn-1) was discovered as a risk gene in X-linked mental retardation (Billuart et al., 1998) and subsequently described as a player in dendritic spine morphogenesis in a Homer/Ophn-1/RhoA pathway (Govek et al., 2004).

Ubiquitination as a posttranslational modification in the regulation of RhoGAPs is a recently noted concept and is extremely scarcely explored. p190GAP was among the first to be described as a UPS-target (Su et al., 2003) and lately identified as a substrate of Cdh1-APC in cell motility (Naoe et al., 2010). In neurons, ubiquitination and proteasomal degradation was first described for the RacGAP alpha1-chimaerin though the E3 ligase is currently unknown (Marland et al., 2011).

The interaction between p190GAP and Cdh1-APC however, could not be reproduced prompting me to search for other brain-abundant GAPs. Among the GAPs tested, p250GAP, a brain-enriched RhoA/Cdc42GAP emerged as a novel interactor of Cdh1-APC. 


\subsection{Growth-promoting p250GAP undergoes activity-modifying ubiquitination}

p250GAP, as has been previously reported, is brain-abundant and is expressed throughout postnatal development (Nakazawa et al., 2003). It is cytosolic given that its major role is to inactivate the cytoskeletal GTPases RhoA and Cdc42 (Nakazawa et al., 2008; Nakazawa et al., 2003). The RhoGAP is an N-methyl D-aspartate (NMDA)-receptor associated regulator of dendritic spine morphology and dendritic plasticity (Nakazawa et al., 2008; Nakazawa et al., 2003; Wayman et al., 2008). Earlier this year, p250GAP was proposed as a susceptibility gene for schizophrenia and schizotypal personality traits (Ohi et al., 2012).

My functional assays revealed an axon growth-promoting role for p250GAP in cerebellar granule neurons. Further, I found that the RhoA GAP operates downstream of Cdh1APC in a linear pathway in axon growth control, however, my results fail to support a proteasomal turnover of p250GAP by Cdh1-APC.

Ubiquitination of proteins may not only target them for proteasomal degradation but also modify the activity or the functional state of a protein (Hicke, 2001), as is the case with other posttranslational modifications viz. phosphorylation or glycosylation. For example, the HECT E3 ligase Nedd4-1 mediates the mono- or di-ubiquitination of the small GTPase, Rap2A. This blocks the activity of Rap2A and its effector kinase and thereby enhances dendritic extension and arborization (Kawabe et al., 2010).

The N-end rule of ubiquitin-mediated proteasomal degradation of proteins can be recapitulated with fusion proteins harboring a non-removable ubiquitin at their $\mathrm{N}$-terminus (Johnson et al., 1995). This is also true of fusion proteins with ubiquitin in the middle of the sequence (Qian et al., 2002). However, fusion proteins with a C-terminal ubiquitin are metabolically stable (Qian et al., 2002).

Consistent with these reports, N-terminal but not C-terminal fusion of ubiquitin destabilizes p250GAP. Interestingly, wild-type p250GAP is also not destabilized and likely associated with mono, di- or multi-ubiquitin in these assays. The very high molecular weight of the protein $(>250 \mathrm{kDa})$ makes it challenging to perceive the individual ubiquitin bands, in the case of di- or multi-ubiquitination. Further analyses will require the use of commercially available mono- and poly-ubiquitination specific antibodies. 
In axon growth assays, none of the two ubiquitin fusion proteins rescues p250GAP knockdown. p250GAP-ubiquitin which is not degraded may be functionally inactive and hence, ineffective in promoting axon growth. Taken together, my results propose that p250GAP undergoes activity-modifying non-destabilizing ubiquitination in axon growth control.

Whether ubiquitin-modification of p250GAP is catalyzed by Cdh1-APC remains an open question. Some evidence of this can be piled up based on the observation that Cdh1interacting C-terminal fragment of p250GAP WT is also the fragment that is ubiquitinated when overexpressed in heterologous cells. Also, in addition to catalyzing chain-ubiquitination, Cdh1-APC is known to mediate mono-ubiquitination of multiple lysine residues in substrates (Dimova et al., 2012). However, owing to the large size of the protein and a correspondingly large number of lysine residues (76), the potential sites of ubiquitination of wild-type p250GAP can only be predicted by mass spectrometry. Here, it is also noteworthy that unlike Smurf1, p250GAP contains only one putative D-box sequence (ㅁGIL) at its N-terminus and does not possess any other Cdh1 recognition motifs reported so far. A p250GAP D-box mutant does not exhibit any loss-of- or reduced Cdh1 interaction nor increased stability.

\subsection{0 p250GAP is indispensible for neuronal development in vivo}

In vivo knockdown of p250GAP in the rat cerebellum results in a failure of granule neuron migration. At P9, viz. 5 days post-electroporation, a significant number of granule neurons are stuck in the EGL/ML as compared to control transfection. Moreover, the p250GAP knockdown processes are underdeveloped and have an abnormal morphology. Owing to aberrant process growth, the p250GAP knockdown neurons do not form characteristic parallel fibers. Overall, p250GAP affects axonal growth and migration in the developing mammalian brain. As with Smurf1, p250GAP may serve to control granule neuron axon growth and migration in vivo by negatively regulating RhoA (Schematic 4B).

\subsection{Smurf1 and p250GAP: a potential cooperative control of RhoA}

Interestingly, in vivo knockdown of both p250GAP and Smurf1 leads to additive effects on neuronal migration but non-additive effects on axonal lengths. The results suggest that Smurf1 and p250GAP may participate in independent pathways in the control of granule neuron migration but may engage in a cross-talk in the regulation of axon growth. 


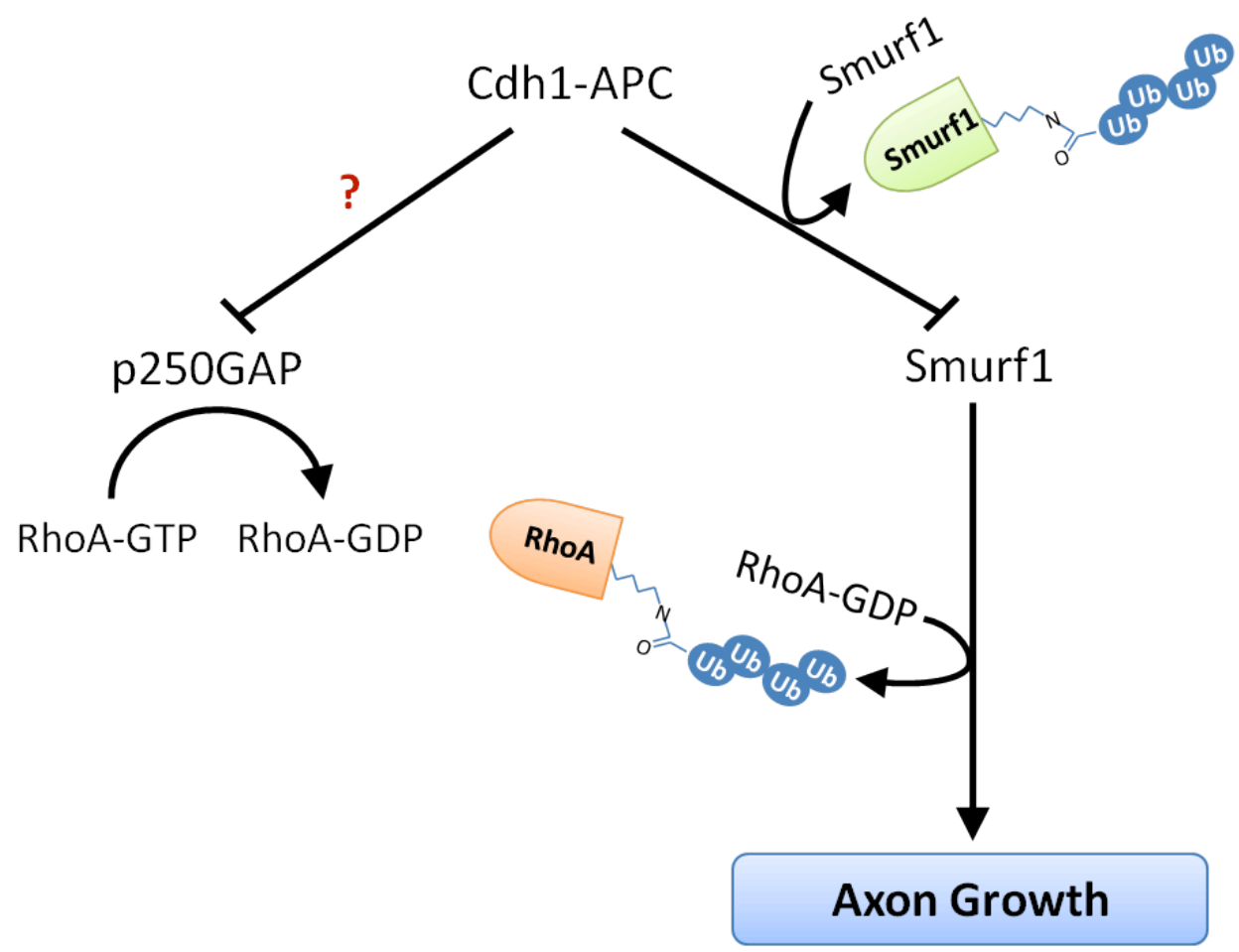

Schematic 4B. p250GAP and Smurf1 operate downstream of Cdh1-APC in axon growth. Both RhoA regulating proteins p250GAP and Smurf1 promote axon growth downstream of Cdh1-APC. Given that Smurf1 preferentially degrades inactive RhoA and that p250GAP inactivates RhoA, it is likely that the two proteins engage in a potential cross-talk in RhoA regulation in axon growth.

Given that Smurf ligases preferentially degrade inactive GTPases, as has been reported for Smurf1-degradation of RhoA (Wang et al., 2003) and Smurf2-degradation of Rap1B (Schwamborn et al., 2007), it is reasonable that Smurfl activity may depend on a RhoGAP that locally inactivates RhoA.

The following in vitro experiments further support the involvement of the two proteins in a shared pathway. Smurf1 and p250GAP physically interact in heterologous cells. In vitro axon assays under Smurf1 and p250GAP double knockdown recapitulate the results obtained in vivo in that there is no additive effect of the RNAi-s on axon lengths in these neurons. Additionally, knockdown of p250GAP overrules Smurf1 DBM3/4-induced axon growth. A possible explanation for this is an inability of Smurf1 (or the stabilized Smurf1 DBM3/4) to degrade 'active' RhoA, which accumulates in the absence of p250GAP.

In addition to inactivating RhoA, p250GAP may serve as a scaffold to locally enrich Smurf1 and facilitate Smurf1-dependent ubiquitination and degradation of RhoA. The potential cooperative engagement of Smurf1 and p250GAP in RhoA degradation requires a careful analysis of total and active RhoA levels under the presence and absence of one or both 
proteins. Besides, it will be worthwhile to assess a possible p250GAP-dependent differential distribution of Smurf1 to axon tips or sites of inactive RhoA.

\subsection{Open questions}

My study has shown that the Cdh1-APC pathway in axon growth control is intertwined with the effectors of extrinsic signaling cascades. Notably, components of the MAPK pathway and the cytoskeletal proteins, RhoA and Cdc42 GTPases are direct mediators of Cdh1-APC in axon growth control. MAPK activity antagonizes Cdh1-growth inhibition while RhoA and Cdc42 are positive regulators of Cdh1-APC activity.

I found that the Cdh1-APC/RhoA pathway involves at least two RhoA regulators Smurf1 and p250GAP. Smurf1 emerged as a novel 'substrate' of Cdh1 and additionally, I characterized the molecular mechanism of Cdh1/Smurf1/RhoA cascade in axon growth. I also uncovered a potential role for Smurf1 DBM3/4, a Cdh1-degradation resistant mutant, in axon regeneration. How the pathway counteracts myelin proteins to permit axon growth after injury, is an open question for the future.

p250GAP also promotes axon growth downstream of Cdh1-APC and undergoes a nondestabilizing ubiquitination, which is possibly accompanied by a loss-of-function in axon growth. My data support the notion that ubiquitination may play a role in the functional modification of a RhoGAP in neuronal development. However, whether this modification is catalyzed by the E3 ligase Cdh1 remains unclear.

My study also postulates Smurf1 and p250GAP in a cooperative control of RhoA downstream of Cdh1 in axon growth control. Both Smurf1 and p250GAP are indispensible for proper axon growth and granule neuron migration in the developing cerebellum and the two proteins participate in a shared pathway in axon growth regulation in vitro and in vivo. Assessing local changes in RhoA in response to Smurf1 and/or p250GAP expression in axon growth and neuronal migration represents a prospective area for future research.

My results together with further studies along these lines will help decipher how Cdh1$\mathrm{APC}$, as an upstream player, orchestrates cytoskeletal dynamics in axon growth and regeneration and from a broader perspective, address how a given cell-intrinsic mechanism can control developmental and regenerative programs. 


\section{Summary}

Neuronal differentiation begins with initiation of the axon. Both extrinsic factors in the neuronal environment and cell-autonomous players contribute to successful axonal morphogenesis. The E3 ubiquitin ligase, Cdh1-Anaphase Promoting Complex (Cdh1-APC) is implicated in cell-intrinsic inhibition of axonal growth in the mammalian brain. In restricting axon growth, Cdh1-APC targets the transcription factors SnoN and Id2 for proteasomal degradation. However, whether the E3 ligase collaborates with extrinsic signaling in controlling axon growth is unknown. In this study, I show that mitogen activated protein kinase (MAPK), phosphatidylinositol 3-kinase (PI3K) and Rho GTPases, effectors of extrinsic trophic signaling, interface with the Cdh1-APC pathway. While MAPK and PI3K activity antagonize Cdh1 inhibition of axon growth, the small Rho GTPases RhoA and Cdc42 are positive mediators of the pathway. A mechanistic insight into the Cdh1-APC/RhoA relationship identified the homologous to E6-AP (HECT) E3 ligase, smad ubiquitination regulatory factor-1 (Smurf1), a negative regulator of RhoA, as a substrate of Cdh1-APC. Smurf1 promotes axon growth downstream of Cdh1-APC by targeting RhoA to proteasomal degradation. Smurf1 binds to Cdh1-APC in a D-box dependent manner and consequently, a stable Smurf1 D-box mutant overcomes Cdh1 inhibition by degrading RhoA and exhibits a gain-of-function in axon growth both on permissive substrates and on myelin. p250 GTPase activating protein (p250GAP), a RhoA inactivator, also participates in the Cdh1-APC pathway alongside Smurf1. Again a positive regulator of axon growth, p250GAP shares a linear pathway downstream of Cdh1-APC. Taken together, my study places Cdh1-APC at the intersection of intrinsic and extrinsic signaling cascades that controls axon growth. Further, my results reiterate an emerging role for the E3 ligase as a suppressor of axon extension and uncover novel candidates for regenerative therapy. 


\section{References}

Allen, M.J., Shan, X., and Murphey, R.K. (2000). A role for Drosophila Drac1 in neurite outgrowth and synaptogenesis in the giant fiber system. Mol Cell Neurosci 16, 754-765.

Almeida, A., Bolanos, J.P., and Moreno, S. (2005). Cdh1/Hct1-APC is essential for the survival of postmitotic neurons. J Neurosci 25, 8115-8121.

Aoki, K., Nakamura, T., and Matsuda, M. (2004). Spatio-temporal regulation of Rac1 and Cdc42 activity during nerve growth factor-induced neurite outgrowth in PC12 cells. J Biol Chem 279, 713-719.

Arevalo, J.C., and Wu, S.H. (2006). Neurotrophin signaling: many exciting surprises! Cell Mol Life Sci 63, 1523-1537.

Atwal, J.K., Massie, B., Miller, F.D., and Kaplan, D.R. (2000). The TrkB-Shc site signals neuronal survival and local axon growth via MEK and P13-kinase. Neuron 27, 265-277.

Barde, Y.A., Edgar, D., and Thoenen, H. (1982). Purification of a new neurotrophic factor from mammalian brain. EMBO J 1, 549-553.

Barresi, V., Ragusa, A., Fichera, M., Musso, N., Castiglia, L., Rappazzo, G., Travali, S., Mattina, T., Romano, C., Cocchi, G., et al. (2010). Decreased expression of GRAF1/OPHN-1$\mathrm{L}$ in the X-linked alpha thalassemia mental retardation syndrome. BMC Med Genomics 3, 28.

Bhakar, A.L., Tannis, L.L., Zeindler, C., Russo, M.P., Jobin, C., Park, D.S., MacPherson, S., and Barker, P.A. (2002). Constitutive nuclear factor-kappa B activity is required for central neuron survival. J Neurosci 22, 8466-8475.

Bilimoria, P.M., and Bonni, A. (2008). Cultures of cerebellar granule neurons. CSH Protoc 2008, pdb prot5107.

Billuart, P., Bienvenu, T., Ronce, N., des Portes, V., Vinet, M.C., Zemni, R., Roest Crollius, H., Carrie, A., Fauchereau, F., Cherry, M., et al. (1998). Oligophrenin-1 encodes a rhoGAP protein involved in X-linked mental retardation. Nature 392, 923-926.

Bingol, B., and Sheng, M. (2011). Deconstruction for reconstruction: the role of proteolysis in neural plasticity and disease. Neuron 69, 22-32.

Boyer, L., Turchi, L., Desnues, B., Doye, A., Ponzio, G., Mege, J.L., Yamashita, M., Zhang, Y.E., Bertoglio, J., Flatau, G., et al. (2006). CNF1-induced ubiquitylation and proteasome destruction of activated RhoA is impaired in Smurf1-/- cells. Mol Biol Cell 17, 2489-2497. 
Brouns, M.R., Matheson, S.F., and Settleman, J. (2001). p190 RhoGAP is the principal Src substrate in brain and regulates axon outgrowth, guidance and fasciculation. Nat Cell Biol 3, 361-367.

Bryan, B., Cai, Y., Wrighton, K., Wu, G., Feng, X.H., and Liu, M. (2005). Ubiquitination of RhoA by Smurf1 promotes neurite outgrowth. FEBS Lett 579, 1015-1019.

Burton, J.L., and Solomon, M.J. (2001). D box and KEN box motifs in budding yeast Hsl1p are required for APC-mediated degradation and direct binding to Cdc20p and Cdh1p. Genes Dev 15, 2381-2395.

Cajal, R.y. (1890). Manual de Anatomia Pathologica General (Handbook of general Anatomical Pathology), Vol 1.

Caroni, P., and Schwab, M.E. (1988). Antibody against myelin-associated inhibitor of neurite growth neutralizes nonpermissive substrate properties of CNS white matter. Neuron 1, 85-96.

Chau, V., Tobias, J.W., Bachmair, A., Marriott, D., Ecker, D.J., Gonda, D.K., and Varshavsky, A. (1989). A multiubiquitin chain is confined to specific lysine in a targeted short-lived protein. Science 243, 1576-1583.

Chen, D.F., Jhaveri, S., and Schneider, G.E. (1995). Intrinsic changes in developing retinal neurons result in regenerative failure of their axons. Proc Natl Acad Sci U S A 92, 7287-7291.

Chen, M.S., Huber, A.B., van der Haar, M.E., Frank, M., Schnell, L., Spillmann, A.A., Christ, F., and Schwab, M.E. (2000). Nogo-A is a myelin-associated neurite outgrowth inhibitor and an antigen for monoclonal antibody IN-1. Nature 403, 434-439.

Cheng, P.L., Lu, H., Shelly, M., Gao, H., and Poo, M.M. (2011). Phosphorylation of E3 ligase Smurf1 switches its substrate preference in support of axon development. Neuron 69, 231-243.

Cosker, K.E., and Eickholt, B.J. (2007). Phosphoinositide 3-kinase signalling events controlling axonal morphogenesis. Biochem Soc Trans 35, 207-210.

Coso, O.A., Chiariello, M., Yu, J.C., Teramoto, H., Crespo, P., Xu, N., Miki, T., and Gutkind, J.S. (1995). The small GTP-binding proteins Rac1 and Cdc42 regulate the activity of the JNK/SAPK signaling pathway. Cell 81, 1137-1146.

Cui, Y., He, S., Xing, C., Lu, K., Wang, J., Xing, G., Meng, A., Jia, S., He, F., and Zhang, L. (2011). SCFFBXL(1)(5) regulates BMP signalling by directing the degradation of HECT-type ubiquitin ligase Smurf1. EMBO J 30, 2675-2689.

Cyr, D.M., Hohfeld, J., and Patterson, C. (2002). Protein quality control: U-box-containing E3 ubiquitin ligases join the fold. Trends Biochem Sci 27, 368-375. 
da Fonseca, P.C., Kong, E.H., Zhang, Z., Schreiber, A., Williams, M.A., Morris, E.P., and Barford, D. (2011). Structures of APC/C(Cdh1) with substrates identify Cdh1 and Apc10 as the D-box co-receptor. Nature 470, 274-278.

Da Silva, J.S., Medina, M., Zuliani, C., Di Nardo, A., Witke, W., and Dotti, C.G. (2003). RhoA/ROCK regulation of neuritogenesis via profilin IIa-mediated control of actin stability. J Cell Biol 162, 1267-1279.

Dergham, P., Ellezam, B., Essagian, C., Avedissian, H., Lubell, W.D., and McKerracher, L. (2002). Rho signaling pathway targeted to promote spinal cord repair. J Neurosci 22, 65706577.

Dickson, B.J. (2001). Rho GTPases in growth cone guidance. Curr Opin Neurobiol 11, 103110.

Dimova, N.V., Hathaway, N.A., Lee, B.H., Kirkpatrick, D.S., Berkowitz, M.L., Gygi, S.P., Finley, D., and King, R.W. (2012). APC/C-mediated multiple monoubiquitylation provides an alternative degradation signal for cyclin B1. Nat Cell Biol 14, 168-176.

Drinjakovic, J., Jung, H., Campbell, D.S., Strochlic, L., Dwivedy, A., and Holt, C.E. (2010). E3 ligase Nedd4 promotes axon branching by downregulating PTEN. Neuron 65, 341-357.

Ebisawa, T., Fukuchi, M., Murakami, G., Chiba, T., Tanaka, K., Imamura, T., and Miyazono, K. (2001). Smurf1 interacts with transforming growth factor-beta type I receptor through Smad7 and induces receptor degradation. J Biol Chem 276, 12477-12480.

Fang, G., Yu, H., and Kirschner, M.W. (1998). Direct binding of CDC20 protein family members activates the anaphase-promoting complex in mitosis and G1. Mol Cell 2, 163-171.

Foe, I., and Toczyski, D. (2011). Structural biology: a new look for the APC. Nature 470, 182183.

Fournier, A.E., GrandPre, T., and Strittmatter, S.M. (2001). Identification of a receptor mediating Nogo-66 inhibition of axonal regeneration. Nature 409, 341-346.

Fu, A.K., Hung, K.W., Fu, W.Y., Shen, C., Chen, Y., Xia, J., Lai, K.O., and Ip, N.Y. (2011). APC $(\mathrm{Cdh} 1)$ mediates EphA4-dependent downregulation of AMPA receptors in homeostatic plasticity. Nat Neurosci 14, 181-189.

Fukunaga, E., Inoue, Y., Komiya, S., Horiguchi, K., Goto, K., Saitoh, M., Miyazawa, K., Koinuma, D., Hanyu, A., and Imamura, T. (2008). Smurf2 induces ubiquitin-dependent degradation of Smurfl to prevent migration of breast cancer cells. J Biol Chem 283, 3566035667.

Ge, W., He, F., Kim, K.J., Blanchi, B., Coskun, V., Nguyen, L., Wu, X., Zhao, J., Heng, J.I., Martinowich, K., et al. (2006). Coupling of cell migration with neurogenesis by proneural bHLH factors. Proc Natl Acad Sci U S A 103, 1319-1324. 
Gieffers, C., Peters, B.H., Kramer, E.R., Dotti, C.G., and Peters, J.M. (1999). Expression of the $\mathrm{CDH} 1$-associated form of the anaphase-promoting complex in postmitotic neurons. Proc Natl Acad Sci U S A 96, 11317-11322.

Glotzer, M., Murray, A.W., and Kirschner, M.W. (1991). Cyclin is degraded by the ubiquitin pathway. Nature 349, 132-138.

Gmachl, M., Gieffers, C., Podtelejnikov, A.V., Mann, M., and Peters, J.M. (2000). The RING$\mathrm{H} 2$ finger protein APC11 and the E2 enzyme UBC4 are sufficient to ubiquitinate substrates of the anaphase-promoting complex. Proc Natl Acad Sci U S A 97, 8973-8978.

Goldberg, D.J., and Burmeister, D.W. (1986). Stages in axon formation: observations of growth of Aplysia axons in culture using video-enhanced contrast-differential interference contrast microscopy. J Cell Biol 103, 1921-1931.

Goldberg, J.L., Klassen, M.P., Hua, Y., and Barres, B.A. (2002). Amacrine-signaled loss of intrinsic axon growth ability by retinal ganglion cells. Science 296, 1860-1864.

Goold, R.G., and Gordon-Weeks, P.R. (2004). Glycogen synthase kinase 3beta and the regulation of axon growth. Biochem Soc Trans 32, 809-811.

Govek, E.E., Hatten, M.E., and Van Aelst, L. (2011). The role of Rho GTPase proteins in CNS neuronal migration. Dev Neurobiol 71, 528-553.

Govek, E.E., Newey, S.E., Akerman, C.J., Cross, J.R., Van der Veken, L., and Van Aelst, L. (2004). The X-linked mental retardation protein oligophrenin-1 is required for dendritic spine morphogenesis. Nat Neurosci 7, 364-372.

Govek, E.E., Newey, S.E., and Van Aelst, L. (2005). The role of the Rho GTPases in neuronal development. Genes Dev 19, 1-49.

GrandPre, T., Nakamura, F., Vartanian, T., and Strittmatter, S.M. (2000). Identification of the Nogo inhibitor of axon regeneration as a Reticulon protein. Nature 403, 439-444.

Groll, M., Bajorek, M., Kohler, A., Moroder, L., Rubin, D.M., Huber, R., Glickman, M.H., and Finley, D. (2000). A gated channel into the proteasome core particle. Nat Struct Biol 7, 10621067.

Groll, M., Ditzel, L., Lowe, J., Stock, D., Bochtler, M., Bartunik, H.D., and Huber, R. (1997). Structure of 20S proteasome from yeast at 2.4 A resolution. Nature 386, 463-471.

Guertin, D.A., and Sabatini, D.M. (2005). An expanding role for mTOR in cancer. Trends Mol Med 11, 353-361.

Hallbook, F., Ibanez, C.F., and Persson, H. (1991). Evolutionary studies of the nerve growth factor family reveal a novel member abundantly expressed in Xenopus ovary. Neuron 6, 845858. 
Hand, R., Bortone, D., Mattar, P., Nguyen, L., Heng, J.I., Guerrier, S., Boutt, E., Peters, E., Barnes, A.P., Parras, C., et al. (2005). Phosphorylation of Neurogenin2 specifies the migration properties and the dendritic morphology of pyramidal neurons in the neocortex. Neuron 48, 4562.

Harada, A., Furuta, B., Takeuchi, K., Itakura, M., Takahashi, M., and Umeda, M. (2000). Nadrin, a novel neuron-specific GTPase-activating protein involved in regulated exocytosis. J Biol Chem 275, 36885-36891.

Harel, N.Y., and Strittmatter, S.M. (2006). Can regenerating axons recapitulate developmental guidance during recovery from spinal cord injury? Nat Rev Neurosci 7, 603-616.

Harper, J.W., Burton, J.L., and Solomon, M.J. (2002). The anaphase-promoting complex: it's not just for mitosis any more. Genes Dev 16, 2179-2206.

Hauf, S., Waizenegger, I.C., and Peters, J.M. (2001). Cohesin cleavage by separase required for anaphase and cytokinesis in human cells. Science 293, 1320-1323.

Hendrickson, C., Meyn, M.A., 3rd, Morabito, L., and Holloway, S.L. (2001). The KEN box regulates $\mathrm{Clb} 2$ proteolysis in $\mathrm{G} 1$ and at the metaphase-to-anaphase transition. Curr Biol 11, 1781-1787.

Herrero-Mendez, A., Almeida, A., Fernandez, E., Maestre, C., Moncada, S., and Bolanos, J.P. (2009). The bioenergetic and antioxidant status of neurons is controlled by continuous degradation of a key glycolytic enzyme by APC/C-Cdh1. Nat Cell Biol 11, 747-752.

Hershko, A., and Ciechanover, A. (1998). The ubiquitin system. Annu Rev Biochem 67, 425479.

Hicke, L. (2001). Protein regulation by monoubiquitin. Nat Rev Mol Cell Biol 2, 195-201.

Hoeffer, C.A., and Klann, E. (2010). mTOR signaling: at the crossroads of plasticity, memory and disease. Trends Neurosci 33, 67-75.

Hoffman, P.N. (2010). A conditioning lesion induces changes in gene expression and axonal transport that enhance regeneration by increasing the intrinsic growth state of axons. Exp Neurol 223, 11-18.

Hsieh, S.H., Ferraro, G.B., and Fournier, A.E. (2006). Myelin-associated inhibitors regulate cofilin phosphorylation and neuronal inhibition through LIM kinase and Slingshot phosphatase. J Neurosci 26, 1006-1015.

Hsu, J.Y., Reimann, J.D., Sorensen, C.S., Lukas, J., and Jackson, P.K. (2002). E2F-dependent accumulation of hEmil regulates S phase entry by inhibiting APC(Cdh1). Nat Cell Biol 4, 358366. 
Huang, E.J., and Reichardt, L.F. (2001). Neurotrophins: roles in neuronal development and function. Annu Rev Neurosci 24, 677-736.

Huang, E.J., and Reichardt, L.F. (2003). Trk receptors: roles in neuronal signal transduction. Annu Rev Biochem 72, 609-642.

Huber, A.B., Weinmann, O., Brosamle, C., Oertle, T., and Schwab, M.E. (2002). Patterns of Nogo mRNA and protein expression in the developing and adult rat and after CNS lesions. $\mathrm{J}$ Neurosci 22, 3553-3567.

Huynh, M.A., Stegmuller, J., Litterman, N., and Bonni, A. (2009). Regulation of Cdh1-APC function in axon growth by Cdh1 phosphorylation. J Neurosci 29, 4322-4327.

Ikeuchi, Y., Stegmuller, J., Netherton, S., Huynh, M.A., Masu, M., Frank, D., Bonni, S., and Bonni, A. (2009). A SnoN-Ccd1 pathway promotes axonal morphogenesis in the mammalian brain. J Neurosci 29, 4312-4321.

Inoue, Y., and Imamura, T. (2008). Regulation of TGF-beta family signaling by E3 ubiquitin ligases. Cancer Sci 99, 2107-2112.

Irniger, S., Piatti, S., Michaelis, C., and Nasmyth, K. (1995). Genes involved in sister chromatid separation are needed for B-type cyclin proteolysis in budding yeast. Cell $81,269-$ 278.

Jacobs, H.W., Keidel, E., and Lehner, C.F. (2001). A complex degradation signal in Cyclin A required for G1 arrest, and a C-terminal region for mitosis. EMBO J 20, 2376-2386.

Jaquenoud, M., van Drogen, F., and Peter, M. (2002). Cell cycle-dependent nuclear export of Cdh1p may contribute to the inactivation of APC/C(Cdh1). EMBO J 21, 6515-6526.

Jarius, S., Wandinger, K.P., Horn, S., Heuer, H., and Wildemann, B. (2010). A new Purkinje cell antibody (anti-Ca) associated with subacute cerebellar ataxia: immunological characterization. J Neuroinflammation 7, 21.

Jaspersen, S.L., Charles, J.F., and Morgan, D.O. (1999). Inhibitory phosphorylation of the APC regulator Hct1 is controlled by the kinase Cdc28 and the phosphatase Cdc14. Curr Biol 9, 227236.

Johnson, E.S., Ma, P.C., Ota, I.M., and Varshavsky, A. (1995). A proteolytic pathway that recognizes ubiquitin as a degradation signal. J Biol Chem 270, 17442-17456.

Juo, P., and Kaplan, J.M. (2004). The anaphase-promoting complex regulates the abundance of GLR-1 glutamate receptors in the ventral nerve cord of C. elegans. Curr Biol 14, 2057-2062.

Jura, N., Scotto-Lavino, E., Sobczyk, A., and Bar-Sagi, D. (2006). Differential modification of Ras proteins by ubiquitination. Mol Cell 21, 679-687. 
Kalil, K., Szebenyi, G., and Dent, E.W. (2000). Common mechanisms underlying growth cone guidance and axon branching. J Neurobiol 44, 145-158.

Kaplan, D.R., Hempstead, B.L., Martin-Zanca, D., Chao, M.V., and Parada, L.F. (1991a). The trk proto-oncogene product: a signal transducing receptor for nerve growth factor. Science 252, 554-558.

Kaplan, D.R., Martin-Zanca, D., and Parada, L.F. (1991b). Tyrosine phosphorylation and tyrosine kinase activity of the trk proto-oncogene product induced by NGF. Nature $350,158-$ 160.

Kaplow, M.E., Korayem, A.H., and Venkatesh, T.R. (2008). Regulation of glia number in Drosophila by Rap/Fzr, an activator of the anaphase-promoting complex, and Loco, an RGS protein. Genetics 178, 2003-2016.

Kawabe, H., and Brose, N. (2011). The role of ubiquitylation in nerve cell development. Nat Rev Neurosci 12, 251-268.

Kawabe, H., Neeb, A., Dimova, K., Young, S.M., Jr., Takeda, M., Katsurabayashi, S., Mitkovski, M., Malakhova, O.A., Zhang, D.E., Umikawa, M., et al. (2010). Regulation of Rap2A by the ubiquitin ligase Nedd4-1 controls neurite development. Neuron 65, 358-372.

Kim, A.H., Puram, S.V., Bilimoria, P.M., Ikeuchi, Y., Keough, S., Wong, M., Rowitch, D., and Bonni, A. (2009). A centrosomal Cdc20-APC pathway controls dendrite morphogenesis in postmitotic neurons. Cell 136, 322-336.

Kim, M.D., Kamiyama, D., Kolodziej, P., Hing, H., and Chiba, A. (2003). Isolation of Rho GTPase effector pathways during axon development. Dev Biol 262, 282-293.

King, R.W., Glotzer, M., and Kirschner, M.W. (1996). Mutagenic analysis of the destruction signal of mitotic cyclins and structural characterization of ubiquitinated intermediates. Mol Biol Cell 7, 1343-1357.

King, R.W., Peters, J.M., Tugendreich, S., Rolfe, M., Hieter, P., and Kirschner, M.W. (1995). A 20 S complex containing CDC27 and CDC16 catalyzes the mitosis-specific conjugation of ubiquitin to cyclin B. Cell 81, 279-288.

Kisselev, A.F., Akopian, T.N., Woo, K.M., and Goldberg, A.L. (1999). The sizes of peptides generated from protein by mammalian 26 and $20 \mathrm{~S}$ proteasomes. Implications for understanding the degradative mechanism and antigen presentation. J Biol Chem 274, 33633371 .

Klein, R., Jing, S.Q., Nanduri, V., O'Rourke, E., and Barbacid, M. (1991). The trk protooncogene encodes a receptor for nerve growth factor. Cell 65, 189-197.

Koegl, M., Hoppe, T., Schlenker, S., Ulrich, H.D., Mayer, T.U., and Jentsch, S. (1999). A novel ubiquitination factor, E4, is involved in multiubiquitin chain assembly. Cell 96, 635-644. 
Konishi, Y., Stegmuller, J., Matsuda, T., Bonni, S., and Bonni, A. (2004). Cdh1-APC controls axonal growth and patterning in the mammalian brain. Science 303, 1026-1030.

Kraft, C., Vodermaier, H.C., Maurer-Stroh, S., Eisenhaber, F., and Peters, J.M. (2005). The WD40 propeller domain of Cdh1 functions as a destruction box receptor for APC/C substrates. Mol Cell 18, 543-553.

Kranenburg, O., Poland, M., Gebbink, M., Oomen, L., and Moolenaar, W.H. (1997). Dissociation of LPA-induced cytoskeletal contraction from stress fiber formation by differential localization of RhoA. J Cell Sci 110 ( Pt 19), 2417-2427.

Kuczera, T., Stilling, R.M., Hsia, H.E., Bahari-Javan, S., Irniger, S., Nasmyth, K., Sananbenesi, F., and Fischer, A. (2011). The anaphase promoting complex is required for memory function in mice. Learn Mem 18, 49-57.

Kuhn, T.B., Brown, M.D., and Bamburg, J.R. (1998). Rac1-dependent actin filament organization in growth cones is necessary for betal-integrin-mediated advance but not for growth on poly-D-lysine. J Neurobiol 37, 524-540.

Lamprecht, R., Farb, C.R., and LeDoux, J.E. (2002). Fear memory formation involves p190 RhoGAP and ROCK proteins through a GRB2-mediated complex. Neuron 36, 727-738.

Lasorella, A., Stegmuller, J., Guardavaccaro, D., Liu, G., Carro, M.S., Rothschild, G., de la Torre-Ubieta, L., Pagano, M., Bonni, A., and Iavarone, A. (2006). Degradation of Id2 by the anaphase-promoting complex couples cell cycle exit and axonal growth. Nature 442, 471-474.

Lehmann, M., Fournier, A., Selles-Navarro, I., Dergham, P., Sebok, A., Leclerc, N., Tigyi, G., and McKerracher, L. (1999). Inactivation of Rho signaling pathway promotes CNS axon regeneration. J Neurosci 19, 7537-7547.

Leverson, J.D., Joazeiro, C.A., Page, A.M., Huang, H., Hieter, P., and Hunter, T. (2000). The APC11 RING-H2 finger mediates E2-dependent ubiquitination. Mol Biol Cell 11, 2315-2325.

Levi-Montalcini, R., and Hamburger, V. (1951). Selective growth stimulating effects of mouse sarcoma on the sensory and sympathetic nervous system of the chick embryo. J Exp Zool 116, $321-361$.

Li, M., Shin, Y.H., Hou, L., Huang, X., Wei, Z., Klann, E., and Zhang, P. (2008). The adaptor protein of the anaphase promoting complex $\mathrm{Cdh} 1$ is essential in maintaining replicative lifespan and in learning and memory. Nat Cell Biol 10, 1083-1089.

Li, M., and Zhang, P. (2009). The function of APC/CCdh1 in cell cycle and beyond. Cell Div $4,2$.

Listovsky, T., Oren, Y.S., Yudkovsky, Y., Mahbubani, H.M., Weiss, A.M., Lebendiker, M., and Brandeis, M. (2004). Mammalian Cdh1/Fzr mediates its own degradation. EMBO J 23, 1619-1626. 
Littlepage, L.E., and Ruderman, J.V. (2002). Identification of a new APC/C recognition domain, the A box, which is required for the Cdh1-dependent destruction of the kinase AuroraA during mitotic exit. Genes Dev 16, 2274-2285.

Littlepage, L.E., Wu, H., Andresson, T., Deanehan, J.K., Amundadottir, L.T., and Ruderman, J.V. (2002). Identification of phosphorylated residues that affect the activity of the mitotic kinase Aurora-A. Proc Natl Acad Sci U S A 99, 15440-15445.

Liu, C.M., Hur, E.M., and Zhou, F.Q. (2012). Coordinating Gene Expression and Axon Assembly to Control Axon Growth: Potential Role of GSK3 Signaling. Front Mol Neurosci 5, 3.

Liu, K., Tedeschi, A., Park, K.K., and He, Z. (2011). Neuronal intrinsic mechanisms of axon regeneration. Annu Rev Neurosci 34, 131-152.

Lonze, B.E., and Ginty, D.D. (2002). Function and regulation of CREB family transcription factors in the nervous system. Neuron 35, 605-623.

Low, L.K., and Cheng, H.J. (2006). Axon pruning: an essential step underlying the developmental plasticity of neuronal connections. Philos Trans R Soc Lond B Biol Sci 361, 1531-1544.

Luo, L. (2000). Rho GTPases in neuronal morphogenesis. Nat Rev Neurosci 1, 173-180.

Luo, L., Hensch, T.K., Ackerman, L., Barbel, S., Jan, L.Y., and Jan, Y.N. (1996). Differential effects of the Rac GTPase on Purkinje cell axons and dendritic trunks and spines. Nature 379, 837-840.

MacDonald, J.I., Gryz, E.A., Kubu, C.J., Verdi, J.M., and Meakin, S.O. (2000). Direct binding of the signaling adapter protein Grb2 to the activation loop tyrosines on the nerve growth factor receptor tyrosine kinase, TrkA. J Biol Chem 275, 18225-18233.

Mackay, D.J., Nobes, C.D., and Hall, A. (1995). The Rho's progress: a potential role during neuritogenesis for the Rho family of GTPases. Trends Neurosci 18, 496-501.

Maestre, C., Delgado-Esteban, M., Gomez-Sanchez, J.C., Bolanos, J.P., and Almeida, A. (2008). Cdk5 phosphorylates Cdh1 and modulates cyclin B1 stability in excitotoxicity. EMBO J 27, 2736-2745.

Maisonpierre, P.C., Belluscio, L., Squinto, S., Ip, N.Y., Furth, M.E., Lindsay, R.M., and Yancopoulos, G.D. (1990). Neurotrophin-3: a neurotrophic factor related to NGF and BDNF. Science 247, 1446-1451.

Marland, J.R., Pan, D., and Buttery, P.C. (2011). Rac GTPase-activating protein (Rac GAP) alpha1-Chimaerin undergoes proteasomal degradation and is stabilized by diacylglycerol signaling in neurons. J Biol Chem 286, 199-207. 
McGee, A.W., and Strittmatter, S.M. (2003). The Nogo-66 receptor: focusing myelin inhibition of axon regeneration. Trends Neurosci 26, 193-198.

McKerracher, L., David, S., Jackson, D.L., Kottis, V., Dunn, R.J., and Braun, P.E. (1994). Identification of myelin-associated glycoprotein as a major myelin-derived inhibitor of neurite growth. Neuron 13, 805-811.

Mi, S., Lee, X., Shao, Z., Thill, G., Ji, B., Relton, J., Levesque, M., Allaire, N., Perrin, S., Sands, B., et al. (2004). LINGO-1 is a component of the Nogo-66 receptor/p75 signaling complex. Nat Neurosci 7, 221-228.

Michaelson, D., Silletti, J., Murphy, G., D'Eustachio, P., Rush, M., and Philips, M.R. (2001). Differential localization of Rho GTPases in live cells: regulation by hypervariable regions and RhoGDI binding. J Cell Biol 152, 111-126.

Miller, J.J., Summers, M.K., Hansen, D.V., Nachury, M.V., Lehman, N.L., Loktev, A., and Jackson, P.K. (2006). Emil stably binds and inhibits the anaphase-promoting complex/cyclosome as a pseudosubstrate inhibitor. Genes Dev 20, 2410-2420.

Miyamoto, Y., Yamauchi, J., Tanoue, A., Wu, C., and Mobley, W.C. (2006). TrkB binds and tyrosine-phosphorylates Tiam1, leading to activation of Rac1 and induction of changes in cellular morphology. Proc Natl Acad Sci U S A 103, 10444-10449.

Monnier, P.P., Sierra, A., Schwab, J.M., Henke-Fahle, S., and Mueller, B.K. (2003). The Rho/ROCK pathway mediates neurite growth-inhibitory activity associated with the chondroitin sulfate proteoglycans of the CNS glial scar. Mol Cell Neurosci 22, 319-330.

Moon, S.Y., and Zheng, Y. (2003). Rho GTPase-activating proteins in cell regulation. Trends Cell Biol 13, 13-22.

Moore, D.L., Blackmore, M.G., Hu, Y., Kaestner, K.H., Bixby, J.L., Lemmon, V.P., and Goldberg, J.L. (2009). KLF family members regulate intrinsic axon regeneration ability. Science 326, 298-301.

Moore, D.L., and Goldberg, J.L. (2011). Multiple transcription factor families regulate axon growth and regeneration. Dev Neurobiol 71, 1186-1211.

Mukhopadhyay, G., Doherty, P., Walsh, F.S., Crocker, P.R., and Filbin, M.T. (1994). A novel role for myelin-associated glycoprotein as an inhibitor of axonal regeneration. Neuron 13, 757767.

Nakamura, T., Komiya, M., Sone, K., Hirose, E., Gotoh, N., Morii, H., Ohta, Y., and Mori, N. (2002). Grit, a GTPase-activating protein for the Rho family, regulates neurite extension through association with the TrkA receptor and N-Shc and CrkL/Crk adapter molecules. Mol Cell Biol 22, 8721-8734. 
Nakano-Kobayashi, A., Kasri, N.N., Newey, S.E., and Van Aelst, L. (2009). The Rho-linked mental retardation protein OPHN1 controls synaptic vesicle endocytosis via endophilin A1. Curr Biol 19, 1133-1139.

Nakazawa, T., Kuriu, T., Tezuka, T., Umemori, H., Okabe, S., and Yamamoto, T. (2008). Regulation of dendritic spine morphology by an NMDA receptor-associated Rho GTPaseactivating protein, p250GAP. J Neurochem 105, 1384-1393.

Nakazawa, T., Watabe, A.M., Tezuka, T., Yoshida, Y., Yokoyama, K., Umemori, H., Inoue, A., Okabe, S., Manabe, T., and Yamamoto, T. (2003). p250GAP, a novel brain-enriched GTPase-activating protein for Rho family GTPases, is involved in the N-methyl-d-aspartate receptor signaling. Mol Biol Cell 14, 2921-2934.

Namekata, K., Harada, C., Taya, C., Guo, X., Kimura, H., Parada, L.F., and Harada, T. (2010). Dock3 induces axonal outgrowth by stimulating membrane recruitment of the WAVE complex. Proc Natl Acad Sci U S A 107, 7586-7591.

Naoe, H., Araki, K., Nagano, O., Kobayashi, Y., Ishizawa, J., Chiyoda, T., Shimizu, T., Yamamura, K., Sasaki, Y., Saya, H., et al. (2010). The anaphase-promoting complex/cyclosome activator Cdh1 modulates Rho GTPase by targeting p190 RhoGAP for degradation. Mol Cell Biol 30, 3994-4005.

Narimatsu, M., Bose, R., Pye, M., Zhang, L., Miller, B., Ching, P., Sakuma, R., Luga, V., Roncari, L., Attisano, L., et al. (2009). Regulation of planar cell polarity by Smurf ubiquitin ligases. Cell 137, 295-307.

Neumann, S., Bradke, F., Tessier-Lavigne, M., and Basbaum, A.I. (2002). Regeneration of sensory axons within the injured spinal cord induced by intraganglionic cAMP elevation. Neuron 34, 885-893.

Neumann, S., and Woolf, C.J. (1999). Regeneration of dorsal column fibers into and beyond the lesion site following adult spinal cord injury. Neuron 23, 83-91.

Nguyen, L., Besson, A., Heng, J.I., Schuurmans, C., Teboul, L., Parras, C., Philpott, A., Roberts, J.M., and Guillemot, F. (2006). p27kip1 independently promotes neuronal differentiation and migration in the cerebral cortex. Genes Dev 20, 1511-1524.

Nusser, N., Gosmanova, E., Zheng, Y., and Tigyi, G. (2002). Nerve growth factor signals through TrkA, phosphatidylinositol 3-kinase, and Rac1 to inactivate RhoA during the initiation of neuronal differentiation of PC12 cells. J Biol Chem 277, 35840-35846.

Ohi, K., Hashimoto, R., Nakazawa, T., Okada, T., Yasuda, Y., Yamamori, H., Fukumoto, M., Umeda-Yano, S., Iwase, M., Kazui, H., et al. (2012). The p250GAP gene is associated with risk for schizophrenia and schizotypal personality traits. PLoS One 7, e35696. 
Ostapenko, D., Burton, J.L., Wang, R., and Solomon, M.J. (2008). Pseudosubstrate inhibition of the anaphase-promoting complex by Acm1: regulation by proteolysis and Cdc28 phosphorylation. Mol Cell Biol 28, 4653-4664.

Ozdamar, B., Bose, R., Barrios-Rodiles, M., Wang, H.R., Zhang, Y., and Wrana, J.L. (2005). Regulation of the polarity protein Par6 by TGFbeta receptors controls epithelial cell plasticity. Science 307, 1603-1609.

Park, K.K., Liu, K., Hu, Y., Smith, P.D., Wang, C., Cai, B., Xu, B., Connolly, L., Kramvis, I., Sahin, M., et al. (2008). Promoting axon regeneration in the adult CNS by modulation of the PTEN/mTOR pathway. Science 322, 963-966.

Patapoutian, A., and Reichardt, L.F. (2001). Trk receptors: mediators of neurotrophin action. Curr Opin Neurobiol 11, 272-280.

Pfleger, C.M., and Kirschner, M.W. (2000). The KEN box: an APC recognition signal distinct from the D box targeted by Cdh1. Genes Dev 14, 655-665.

Prinjha, R., Moore, S.E., Vinson, M., Blake, S., Morrow, R., Christie, G., Michalovich, D., Simmons, D.L., and Walsh, F.S. (2000). Inhibitor of neurite outgrowth in humans. Nature 403, 383-384.

Prinz, S., Hwang, E.S., Visintin, R., and Amon, A. (1998). The regulation of Cdc20 proteolysis reveals a role for APC components $\mathrm{Cdc} 23$ and $\mathrm{Cdc} 27$ during $\mathrm{S}$ phase and early mitosis. Curr Biol 8, 750-760.

Qian, S.B., Ott, D.E., Schubert, U., Bennink, J.R., and Yewdell, J.W. (2002). Fusion proteins with $\mathrm{COOH}$-terminal ubiquitin are stable and maintain dual functionality in vivo. J Biol Chem $277,38818-38826$.

Qiu, J., Cai, D., Dai, H., McAtee, M., Hoffman, P.N., Bregman, B.S., and Filbin, M.T. (2002). Spinal axon regeneration induced by elevation of cyclic AMP. Neuron 34, 895-903.

Reid, T., Furuyashiki, T., Ishizaki, T., Watanabe, G., Watanabe, N., Fujisawa, K., Morii, N., Madaule, P., and Narumiya, S. (1996). Rhotekin, a new putative target for Rho bearing homology to a serine/threonine kinase, PKN, and rhophilin in the rho-binding domain. J Biol Chem 271, 13556-13560.

Reimann, J.D., Gardner, B.E., Margottin-Goguet, F., and Jackson, P.K. (2001). Emil regulates the anaphase-promoting complex by a different mechanism than Mad2 proteins. Genes Dev 15, 3278-3285.

Reis, A., Levasseur, M., Chang, H.Y., Elliott, D.J., and Jones, K.T. (2006). The CRY box: a second APCcdh1-dependent degron in mammalian cdc20. EMBO Rep 7, 1040-1045.

Ren, X.D., Kiosses, W.B., and Schwartz, M.A. (1999). Regulation of the small GTP-binding protein Rho by cell adhesion and the cytoskeleton. EMBO J 18, 578-585. 
Rodriguez-Rodriguez, P., Fernandez, E., Almeida, A., and Bolanos, J.P. (2012). Excitotoxic stimulus stabilizes PFKFB3 causing pentose-phosphate pathway to glycolysis switch and neurodegeneration. Cell Death Differ.

Rotin, D., and Kumar, S. (2009). Physiological functions of the HECT family of ubiquitin ligases. Nat Rev Mol Cell Biol 10, 398-409.

Sahai, E., Garcia-Medina, R., Pouyssegur, J., and Vial, E. (2007). Smurf1 regulates tumor cell plasticity and motility through degradation of RhoA leading to localized inhibition of contractility. J Cell Biol 176, 35-42.

Sambrook, J., and Russell, D.W. (2006). Calcium-phosphate-mediated Transfection of Eukaryotic Cells with Plasmid DNAs. CSH Protoc 2006.

Sander, E.E., van Delft, S., ten Klooster, J.P., Reid, T., van der Kammen, R.A., Michiels, F., and Collard, J.G. (1998). Matrix-dependent Tiam1/Rac signaling in epithelial cells promotes either cell-cell adhesion or cell migration and is regulated by phosphatidylinositol 3-kinase. $\mathrm{J}$ Cell Biol 143, 1385-1398.

Sarner, S., Kozma, R., Ahmed, S., and Lim, L. (2000). Phosphatidylinositol 3-kinase, Cdc42, and Rac1 act downstream of Ras in integrin-dependent neurite outgrowth in N1E-115 neuroblastoma cells. Mol Cell Biol 20, 158-172.

Sato, Y., and Heuckeroth, R.O. (2008). Retinoic acid regulates murine enteric nervous system precursor proliferation, enhances neuronal precursor differentiation, and reduces neurite growth in vitro. Dev Biol 320, 185-198.

Schreiber, A., Stengel, F., Zhang, Z., Enchev, R.I., Kong, E.H., Morris, E.P., Robinson, C.V., da Fonseca, P.C., and Barford, D. (2011). Structural basis for the subunit assembly of the anaphase-promoting complex. Nature 470, 227-232.

Schwab, M., Lutum, A.S., and Seufert, W. (1997). Yeast Hct1 is a regulator of Clb2 cyclin proteolysis. Cell 90, 683-693.

Schwab, M.E. (1993). Experimental aspects of spinal cord regeneration. Curr Opin Neurol Neurosurg 6, 549-553.

Schwab, M.E. (2004). Nogo and axon regeneration. Curr Opin Neurobiol 14, 118-124.

Schwab, M.E., Kapfhammer, J.P., and Bandtlow, C.E. (1993). Inhibitors of neurite growth. Annu Rev Neurosci 16, 565-595.

Schwamborn, J.C., Muller, M., Becker, A.H., and Puschel, A.W. (2007). Ubiquitination of the GTPase Rap1B by the ubiquitin ligase Smurf2 is required for the establishment of neuronal polarity. EMBO J 26, 1410-1422. 
Sczaniecka, M., Feoktistova, A., May, K.M., Chen, J.S., Blyth, J., Gould, K.L., and Hardwick, K.G. (2008). The spindle checkpoint functions of Mad3 and Mad2 depend on a Mad3 KEN box-mediated interaction with Cdc20-anaphase-promoting complex (APC/C). J Biol Chem 283, 23039-23047.

Shirayama, M., Toth, A., Galova, M., and Nasmyth, K. (1999). APC(Cdc20) promotes exit from mitosis by destroying the anaphase inhibitor Pds1 and cyclin Clb5. Nature 402, 203-207.

Silies, M., and Klambt, C. (2010). APC/C(Fzr/Cdh1)-dependent regulation of cell adhesion controls glial migration in the Drosophila PNS. Nat Neurosci 13, 1357-1364.

Smith, D.M., Kafri, G., Cheng, Y., Ng, D., Walz, T., and Goldberg, A.L. (2005). ATP binding to PAN or the 26S ATPases causes association with the 20S proteasome, gate opening, and translocation of unfolded proteins. Mol Cell 20, 687-698.

Stegmuller, J., Huynh, M.A., Yuan, Z., Konishi, Y., and Bonni, A. (2008). TGFbeta-Smad2 signaling regulates the Cdh1-APC/SnoN pathway of axonal morphogenesis. J Neurosci 28, 1961-1969.

Stegmuller, J., Konishi, Y., Huynh, M.A., Yuan, Z., Dibacco, S., and Bonni, A. (2006). Cellintrinsic regulation of axonal morphogenesis by the Cdh1-APC target SnoN. Neuron 50, 389400 .

Stroschein, S.L., Bonni, S., Wrana, J.L., and Luo, K. (2001). Smad3 recruits the anaphasepromoting complex for ubiquitination and degradation of SnoN. Genes Dev 15, 2822-2836.

Su, L., Agati, J.M., and Parsons, S.J. (2003). p190RhoGAP is cell cycle regulated and affects cytokinesis. J Cell Biol 163, 571-582.

Tajima, Y., Goto, K., Yoshida, M., Shinomiya, K., Sekimoto, T., Yoneda, Y., Miyazono, K., and Imamura, T. (2003). Chromosomal region maintenance 1 (CRM1)-dependent nuclear export of Smad ubiquitin regulatory factor 1 (Smurf1) is essential for negative regulation of transforming growth factor-beta signaling by Smad7. J Biol Chem 278, 10716-10721.

Tang, Z., Li, B., Bharadwaj, R., Zhu, H., Ozkan, E., Hakala, K., Deisenhofer, J., and Yu, H. (2001). APC2 Cullin protein and APC11 RING protein comprise the minimal ubiquitin ligase module of the anaphase-promoting complex. Mol Biol Cell 12, 3839-3851.

Tcherkezian, J., and Lamarche-Vane, N. (2007). Current knowledge of the large RhoGAP family of proteins. Biol Cell 99, 67-86.

Thornton, B.R., Ng, T.M., Matyskiela, M.E., Carroll, C.W., Morgan, D.O., and Toczyski, D.P. (2006). An architectural map of the anaphase-promoting complex. Genes Dev 20, 449-460.

Trakhtenberg, E.F., and Goldberg, J.L. (2012). Epigenetic regulation of axon and dendrite growth. Front Mol Neurosci 5, 24. 
Tursun, B., Schluter, A., Peters, M.A., Viehweger, B., Ostendorff, H.P., Soosairajah, J., Drung, A., Bossenz, M., Johnsen, S.A., Schweizer, M., et al. (2005). The ubiquitin ligase Rnf6 regulates local LIM kinase 1 levels in axonal growth cones. Genes Dev 19, 2307-2319.

Uhlmann, F., Lottspeich, F., and Nasmyth, K. (1999). Sister-chromatid separation at anaphase onset is promoted by cleavage of the cohesin subunit Scc1. Nature 400, 37-42.

van Roessel, P., Elliott, D.A., Robinson, I.M., Prokop, A., and Brand, A.H. (2004). Independent regulation of synaptic size and activity by the anaphase-promoting complex. Cell 119, 707-718.

Vargas, M.E., and Barres, B.A. (2007). Why is Wallerian degeneration in the CNS so slow? Annu Rev Neurosci 30, 153-179.

Verma, R., Aravind, L., Oania, R., McDonald, W.H., Yates, J.R., 3rd, Koonin, E.V., and Deshaies, R.J. (2002). Role of Rpn11 metalloprotease in deubiquitination and degradation by the 26S proteasome. Science 298, 611-615.

Visintin, R., Craig, K., Hwang, E.S., Prinz, S., Tyers, M., and Amon, A. (1998). The phosphatase $\mathrm{Cdc} 14$ triggers mitotic exit by reversal of Cdk-dependent phosphorylation. Mol Cell 2, 709-718.

Vodermaier, H.C., Gieffers, C., Maurer-Stroh, S., Eisenhaber, F., and Peters, J.M. (2003). TPR subunits of the anaphase-promoting complex mediate binding to the activator protein CDH1. Curr Biol 13, 1459-1468.

Vohra, B.P., Fu, M., and Heuckeroth, R.O. (2007). Protein kinase Czeta and glycogen synthase kinase-3beta control neuronal polarity in developing rodent enteric neurons, whereas SMAD specific E3 ubiquitin protein ligase 1 promotes neurite growth but does not influence polarity. J Neurosci 27, 9458-9468.

Wan, L., Zou, W., Gao, D., Inuzuka, H., Fukushima, H., Berg, A.H., Drapp, R., Shaik, S., Hu, D., Lester, C., et al. (2011). Cdh1 regulates osteoblast function through an APC/C-independent modulation of Smurf1. Mol Cell 44, 721-733.

Wan, Y., Liu, X., and Kirschner, M.W. (2001). The anaphase-promoting complex mediates TGF-beta signaling by targeting SnoN for destruction. Mol Cell 8, 1027-1039.

Wang, H.R., Zhang, Y., Ozdamar, B., Ogunjimi, A.A., Alexandrova, E., Thomsen, G.H., and Wrana, J.L. (2003). Regulation of cell polarity and protrusion formation by targeting RhoA for degradation. Science 302, 1775-1779.

Wang, K.C., Kim, J.A., Sivasankaran, R., Segal, R., and He, Z. (2002). P75 interacts with the Nogo receptor as a co-receptor for Nogo, MAG and OMgp. Nature 420, 74-78.

Wayman, G.A., Davare, M., Ando, H., Fortin, D., Varlamova, O., Cheng, H.Y., Marks, D., Obrietan, K., Soderling, T.R., Goodman, R.H., et al. (2008). An activity-regulated microRNA 
controls dendritic plasticity by down-regulating p250GAP. Proc Natl Acad Sci U S A 105, 9093-9098.

Weissman, A.M. (2001). Themes and variations on ubiquitylation. Nat Rev Mol Cell Biol 2, 169-178.

Welchman, R.L., Gordon, C., and Mayer, R.J. (2005). Ubiquitin and ubiquitin-like proteins as multifunctional signals. Nat Rev Mol Cell Biol 6, 599-609.

Yamaguchi, K., Ohara, O., Ando, A., and Nagase, T. (2008). Smurf1 directly targets hPEM-2, a GEF for $\mathrm{Cdc} 42$, via a novel combination of protein interaction modules in the ubiquitinproteasome pathway. Biol Chem 389, 405-413.

Yamaguchi, Y., Katoh, H., Yasui, H., Mori, K., and Negishi, M. (2001). RhoA inhibits the nerve growth factor-induced Racl activation through Rho-associated kinase-dependent pathway. J Biol Chem 276, 18977-18983.

Yamashita, M., Ying, S.X., Zhang, G.M., Li, C., Cheng, S.Y., Deng, C.X., and Zhang, Y.E. (2005). Ubiquitin ligase Smurf1 controls osteoblast activity and bone homeostasis by targeting MEKK2 for degradation. Cell 121, 101-113.

Yamashita, T., and Tohyama, M. (2003). The p75 receptor acts as a displacement factor that releases Rho from Rho-GDI. Nat Neurosci 6, 461-467.

Yang, P., and Yang, Z. (2012). Enhancing intrinsic growth capacity promotes adult CNS regeneration. J Neurol Sci 312, 1-6.

Yao, T., and Cohen, R.E. (2002). A cryptic protease couples deubiquitination and degradation by the proteasome. Nature 419, 403-407.

Yiu, G., and He, Z. (2006). Glial inhibition of CNS axon regeneration. Nat Rev Neurosci 7, 617-627.

Yoneda, Y., Hieda, M., Nagoshi, E., and Miyamoto, Y. (1999). Nucleocytoplasmic protein transport and recycling of Ran. Cell Struct Funct 24, 425-433.

Yu, P., Zhang, Y.P., Shields, L.B., Zheng, Y., Hu, X., Hill, R., Howard, R., Gu, Z., Burke, D.A., Whittemore, S.R., et al. (2011). Inhibitor of DNA binding 2 promotes sensory axonal growth after SCI. Exp Neurol 231, 38-44.

Zachariae, W., Schwab, M., Nasmyth, K., and Seufert, W. (1998). Control of cyclin ubiquitination by CDK-regulated binding of Hctl to the anaphase promoting complex. Science $282,1721-1724$.

Zhang, H., and Macara, I.G. (2008). The PAR-6 polarity protein regulates dendritic spine morphogenesis through p190 RhoGAP and the Rho GTPase. Dev Cell 14, 216-226. 
Zhang, Y., Yao, W., Qiu, J., Qian, W., Zhu, C., and Zhang, C. (2011). The involvement of down-regulation of Cdh1-APC in hippocampal neuronal apoptosis after global cerebral ischemia in rat. Neurosci Lett 505, 71-75.

Zhu, H., Kavsak, P., Abdollah, S., Wrana, J.L., and Thomsen, G.H. (1999). A SMAD ubiquitin ligase targets the BMP pathway and affects embryonic pattern formation. Nature 400, 687-693.

Zur, A., and Brandeis, M. (2001). Securin degradation is mediated by fzy and fzr, and is required for complete chromatid separation but not for cytokinesis. EMBO J 20, 792-801. 


\section{Appendix I - Plasmids}

\begin{tabular}{|c|c|}
\hline Plasmid & $\underline{\text { Source/Constructed by }}$ \\
\hline $\begin{array}{l}\text { pCMV5-Flag-Smurf1, } \\
\text { pCMV5-HA-Renilla-Smurf1 WT, } \\
\text { pCMV5-HA-Renilla-Smurf1 DBM, } \\
\text { pBluescript U6/Smurf1 RNAi, } \\
\text { pBluescript U6/p250GAP RNAi, } \\
\text { pCMV-Myc-p250GAP-N, } \\
\text { pCMV-Myc-p250GAP-C }\end{array}$ & Dr. Judith Stegmüller, MPI-EM \\
\hline $\begin{array}{l}\text { RhoA, Rac } 1 \text { and } \text { Cdc } 42 \text { constructs in } \\
\text { pCEFL }\end{array}$ & $\begin{array}{l}\text { Prof. Sylvio Gutkind, National Institutes of } \\
\text { Health, Bathesda, MD, USA }\end{array}$ \\
\hline pCMV5-HA-Smurf1 & $\begin{array}{l}\text { Prof. Joan Massagué, Memorial Sloan-Kettering } \\
\text { Cancer Center, New York, NY, USA }\end{array}$ \\
\hline pCMV-HIS-ubiquitin & $\begin{array}{l}\text { Prof. Wade Harper, Harvard Medical School, } \\
\text { Boston, MA, USA }\end{array}$ \\
\hline pCMV5B-Flag-Smurf2 & Prof. Jeffrey Wrana via Addgene \\
\hline $\begin{array}{l}\text { pCDNA3-6xMyc-p250GAP, } \\
\text { pEGFP-p250GAP (mouse and human) }\end{array}$ & $\begin{array}{l}\text { Dr. Takanobu Nakazawa, Institute of Medical } \\
\text { Science, University of Tokyo, Tokyo, Japan }\end{array}$ \\
\hline $\begin{array}{l}\text { pCDNA3-6xMyc-ubiquitin-p250GAP, } \\
\text { pCDNA3-6xMyc-p250GAP-ubiquitin }\end{array}$ & $\begin{array}{l}\text { Dr. Judith Stegmüller, Nicola Schwedhelm, } \\
\text { Madhuvanthi Kannan, MPI-EM }\end{array}$ \\
\hline $\begin{array}{l}\text { Smurf1 RNAi-CMV-EGFP, } \\
\text { p250GAP RNAi-CMV-EGFP }\end{array}$ & Shih-Ju Lee, MPI-EM \\
\hline pKH3-HA-p190GAP & $\begin{array}{l}\text { Prof. S. J. Parsons, University of Virginia, VA, } \\
\text { USA }\end{array}$ \\
\hline Myc-Ophn1 & $\begin{array}{l}\text { Prof. Linda Van Aelst, Cold Spring Harbor } \\
\text { Laboratory, Cold Spring Harbor, NY, USA }\end{array}$ \\
\hline pTB701-HA-Graf & $\begin{array}{l}\text { Prof. Yoshitaka Ono, Biosignal Research Center, } \\
\text { Kobe University, Kobe, Japan }\end{array}$ \\
\hline pEGFP-Nadrin & $\begin{array}{l}\text { Prof. Utako Kato, Institute of Chemical Research, } \\
\text { Kyoto University, Kyoto, Japan }\end{array}$ \\
\hline
\end{tabular}




\section{Appendix II - Primers}

\begin{tabular}{|c|c|c|}
\hline Construct & $\underline{\text { Primers }}$ & $\underline{\text { Restriction sites }}$ \\
\hline Smurfl (RT-PCR) & $\begin{array}{l}\text { Sense: AAGGCTCTGCAAGGCGCGGC } \\
\text { Antisense: GGTTTCCTCCACCGCTGTCAGC }\end{array}$ & $\overline{--}$ \\
\hline Cdh1 (wild-type) (PCR) & $\begin{array}{l}\text { Sense: ACTCCGCACTGCTGAAGAAT } \\
\text { Antisense: CCAGTCCACCAAGTTGAGGT }\end{array}$ & -- \\
\hline $\begin{array}{l}\text { Cdhl (heterozygote) } \\
\text { (PCR) }\end{array}$ & $\begin{array}{l}\text { Sense: TTGGTTTTCGGGACCTGGGAC } \\
\text { Antisense: CACAAGGGGCTCTTTACG }\end{array}$ & -- \\
\hline $\begin{array}{l}\text { pCMV5-HA-Smurf1- } \\
\text { DBM1 (SDM) }\end{array}$ & $\begin{array}{l}\text { Sense: } \\
\text { CCGGATACCAGGCTTTGGATGCATGCAAACTAGCCCCCTCAGATACTGATGC } \\
\text { Antisense: } \\
\text { GCATCAGTATCTGAGGGGGCTAGTTTGCATGCATCCAAAGCCTGGTATCCGG }\end{array}$ & -- \\
\hline $\begin{array}{l}\text { pCMV5-HA-Smurf1- } \\
\text { DBM2 (SDM) }\end{array}$ & $\begin{array}{l}\text { Sense: GCTCGGTGGTGGACTGCGCAGGACTGGCAGAAAATGAAGGAACG } \\
\text { Antisense: CGTTCCTTCATTTTCTGCCAGTCCTGCGCAGTCCACCACCGAGC }\end{array}$ & -- \\
\hline $\begin{array}{l}\text { pCMV5-HA-Smurf1- } \\
\text { DBM3 (SDM) }\end{array}$ & $\begin{array}{l}\text { Sense: GCTGAAAGTCCTCGCACACGAAGCCTCGCTTCAGCAGCCCCAAG } \\
\text { Antisense: CTTGGGGCTGCTGAAGCGAGGCTTCGTGTGCGAGGACTTTCAGC }\end{array}$ & -- \\
\hline $\begin{array}{l}\text { pCMV5-HA-Smurf1- } \\
\text { DBM4 (SDM) }\end{array}$ & $\begin{array}{l}\text { Sense: GGTGGTGTGGCCGCCGAGTGGGCTTACTTGCTGTGCCATG } \\
\text { Antisense: CATGGCACAGCAAGTAAGCCCACTCGGCGGCCACACCACC }\end{array}$ & -- \\
\hline
\end{tabular}




\begin{tabular}{|c|c|c|}
\hline $\begin{array}{l}\text { pCMV5-HA-Smurf1- } \\
\text { DBM5 (SDM) }\end{array}$ & $\begin{array}{l}\text { Sense: GACTGGGTCCACGGCAGTCCCGGCCCAAGGCTTCAAG } \\
\text { Antisense: CTTGAAGCCTTGGGCCGGGACTGCCGTGGACCCAGTC }\end{array}$ & - \\
\hline $\begin{array}{l}\text { pCMV5-HA-Smurf1-Res } \\
\text { (SDM) }\end{array}$ & $\begin{array}{l}\text { Sense: GATACCGGATACCAGCGTTTAGACCTCTGTAAACTAAACCCCTCAG } \\
\text { Antisense: CTGAGGGGTTTAGTTTACAGAGGTCTAAACGCTGGTATCCGGTATC }\end{array}$ & -- \\
\hline $\begin{array}{l}\text { pCMV5-HA-NES- } \\
\text { Smurf1-Res }\end{array}$ & $\begin{array}{l}\text { Sense: } \\
\text { AATTCATGCTTCAGCTACCACCGCTTGAGAGACTTACTCTTGATCTTCAGCTA } \\
\text { CCACCGCTTGAGAGACTTACTCTTGATGGTAC } \\
\text { Antisense: } \\
\text { CATCAAGAGTAAGTCTCTCAAGCGGTGGTAGCTGAAGATCAAGAGTAAGTCT } \\
\text { CTCAAGCGGTGGTAGCTGAAGCATG }\end{array}$ & EcoRI, KpnI \\
\hline $\begin{array}{l}\text { pCMV5-HA-NLS- } \\
\text { Smurf1-Res }\end{array}$ & $\begin{array}{l}\text { Sense: } \\
\text { AATTCATGGATCCAAAAAAGAAGAGAAAGGTAGATCCAAAAAAGAAGAGAAA } \\
\text { GGTAGGTAC } \\
\text { Antisense: } \\
\text { CTACCTTTCTCTTCTTTTTTGGATCTACCTTTCTCTTCTTTTTTGGATCCATG }\end{array}$ & EcoRI, KpnI \\
\hline $\begin{array}{l}\text { pCMV5-HA-NLS- } \\
\text { Smurf1-Res/NES mutant } \\
\text { (SDM) }\end{array}$ & $\begin{array}{l}\text { Sense: GGCGGCCTGGATAAAGCAGACGCCAACGACTGGAAGTCGAAC } \\
\text { Antisense: GTTCGACTTCCAGTCGTTGGCGTCTGCTTTATCCAGGCCGCC }\end{array}$ & - \\
\hline pCEFL-RhoA K6,7R & $\begin{array}{l}\text { Sense: GGCTGCCATCCGGAGGAGACTGGTGATTGTTGGTG } \\
\text { Antisense: CACCAACAATCACCAGTCTCCTCCGGATGGCAGCC }\end{array}$ & -- \\
\hline $\begin{array}{l}\text { pCDNA3-6xMyc- } \\
\text { p250GAP DBM (SDM) }\end{array}$ & $\begin{array}{l}\text { Sense: CAGAAGCTGAAGCAGGCGGGAATCGCGAAAGAGAGGGTGTTTG } \\
\text { Antisense: CAAACACCCTCTCTTTCGCGATTCCCGCCTGCTTCAGCTTCTG }\end{array}$ & -- \\
\hline
\end{tabular}




\begin{tabular}{|c|c|c|}
\hline $\begin{array}{l}\text { Ubiquitin-p250GAP } \\
\text { cloning, fragment A }\end{array}$ & $\begin{array}{l}\text { Sense: TAATACGACTCACTATAGGG (T7 promoter primer) } \\
\text { Antisense: ATATGACAAGTGTCTCATCAGGAACTC }\end{array}$ & $\begin{array}{r}\text { PshAI } \\
\text { (antisense) }\end{array}$ \\
\hline $\begin{array}{l}\text { pGEM }{ }^{\circledR} \text { T Easy/PstI site } \\
\text { mutant }(\mathrm{SDM})\end{array}$ & $\begin{array}{l}\text { Sense: GAATTCGCGGCCGCCAAAAGGTCGACCATATGGGAGAGCTC } \\
\text { Antisense: GAGCTCTCCCATATGGTCGACCTTTTGGCGGCCGCGAATTC }\end{array}$ & -- \\
\hline $\begin{array}{l}\text { Human ubiquitin primers } \\
\text { (PCR) }\end{array}$ & $\begin{array}{l}\text { Sense: ATACTGCAGAATGCAGATCTTCGTGAAGAC } \\
\text { Antisense: ATACTGCAGTTCCCACCTCTGAGACGG }\end{array}$ & PstI \\
\hline $\begin{array}{l}\text { p250GAP-ubiquitin } \\
\text { cloning, fragment B }\end{array}$ & $\begin{array}{l}\text { Sense: ATATGTACAGTTATGCTGGTTTGGC } \\
\text { Antisense: ATATCCTTAAGTACTAACTTTTAAAAGTCC }\end{array}$ & BsrGI, AflII \\
\hline $\begin{array}{l}\text { Fragment B, stop codon } \\
\text { mutant/ replacement with } \\
\text { HpaI site (SDM) }\end{array}$ & $\begin{array}{l}\text { Sense: } \\
\text { CAGATCCATGCAGAAGTTAACCTGCGAGCAATAGAGTTGAAGCAGCCTC } \\
\text { Antisense: } \\
\text { GAGGCTGCTTCAACTCTATTGCTCGCAGGTTAACTTCTGCATGGATCTG }\end{array}$ & -- \\
\hline $\begin{array}{l}\text { Human ubiquitin primers } \\
\text { (PCR) }\end{array}$ & $\begin{array}{l}\text { Sense: ATAGTTAACATGCAGATCTTCGTGAAGAC } \\
\text { Antisense: TATGTTAACTTACCCACCTCTGAGACGG }\end{array}$ & HpaI \\
\hline
\end{tabular}




\section{Appendix III - Antibodies}

\begin{tabular}{|c|c|}
\hline Antibody & Source \\
\hline $\begin{array}{l}\text { Total Akt rabbit mAb, } \\
\text { phospho Ser }{ }^{273} \text { Akt rabbit mAb, } \\
\text { phospho-Erk1/2 rabbit mAb, } \\
\text { total RhoA rabbit mAb }\end{array}$ & Cell Signaling \\
\hline Total Erk1/2 rabbit pAb & Promega \\
\hline Cdh1 mouse mAb & Santa Cruz and Abcam \\
\hline $\begin{array}{l}\text { SnoN rabbit } \mathrm{pAb}, \\
\text { 14-3-3ß mouse mAb, } \\
\text { Cdc } 27 \text { mouse } \mathrm{mAb}, \\
\text { Smurf1 mouse mAb, } \\
\text { c-myc mouse mAb, } \\
\text { Ubiquitin (P4D1) mouse mAb }\end{array}$ & Santa Cruz \\
\hline $\begin{array}{l}\gamma \text {-tubulin mouse } \mathrm{mAb} \\
\text { Flag mouse } \mathrm{mAb}\end{array}$ & Sigma Aldrich \\
\hline HA mouse $\mathrm{mAb}$ & Covance, Munich \\
\hline GFP rabbit antiserum & Home-made \\
\hline p250GAP rabbit antiserum & $\begin{array}{l}\text { Dr. Takanobu Nakazawa, Institute of Medical } \\
\text { Science, University of Tokyo, Tokyo, Japan }\end{array}$ \\
\hline
\end{tabular}




\section{Appendix IV - GAP Screen}

\begin{tabular}{ll}
\hline Brain-abundant RhoGAP & Neuronal Significance \\
\hline \hline Graf/ARHGAP-26 & $\begin{array}{l}\text { Immunological target in subacute cerebellar ataxia (Jarius et } \\
\text { al., 2010), X-linked alpha-thalessemia mental retardation } \\
\text { (Barresi et al., 2010) }\end{array}$ \\
\hline Nadrin/ARHGAP-17 & $\begin{array}{l}\text { Calcium-dependent exocytosis in nerve endings (Harada et } \\
\text { al., 2000) }\end{array}$ \\
\hline Oligophrenin-1 & $\begin{array}{l}\text { X-linked mental retardation (Billuart et al., 1998); dendritic } \\
\text { spine morphology (Govek et al., 2004); synapse maturation } \\
\text { and plasticity (Nakano-Kobayashi et al., 2009) }\end{array}$ \\
\hline p190GAP & $\begin{array}{l}\text { Axon guidance, axon fasciculation in the CNS (Brouns et } \\
\text { al., 2001); dendritic spine number (Zhang and Macara, } \\
\text { 2008); long-term memory (Lamprecht et al., 2002) }\end{array}$ \\
\hline p250GAP/Grit & $\begin{array}{l}\text { NMDA-receptor associated regulation of dendritic spine } \\
\text { morphology and dendritic plasticity (Nakazawa et al., 2003; } \\
\text { Wayman et al., 2008) }\end{array}$ \\
\hline
\end{tabular}



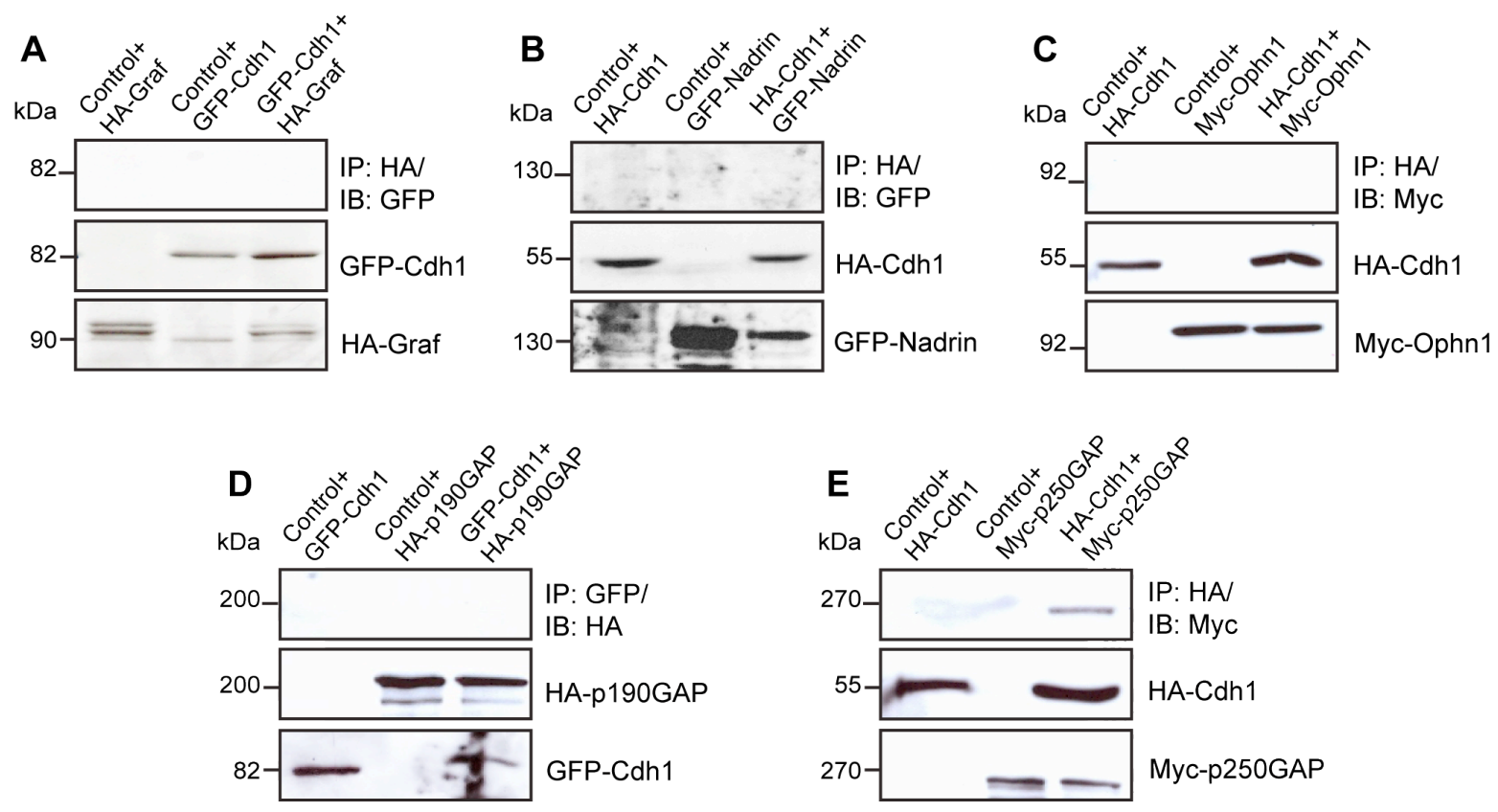

GAP Screen. A. Lysates of 293T transfected with control vector pEGFP and HA-Graf, pTB701 and GFP-Cdh1 or HA-Graf and GFP-Cdh1 plasmids were subjected to immunoprecipitation with the HA antibody followed by immunoblotting with GFP antibody. B. Lysates of $293 \mathrm{~T}$ transfected with control vector pEGFP and HA-Cdh1, pCMV5 and GFP-Nadrin or HA-Cdh1 and GFP-Nadrin plasmids were subjected to immunoprecipitation with the HA antibody followed by immunoblotting with GFP antibody. C. Lysates of 293T transfected with control vector pCDNA3.1 and HA-Cdh1, pCMV5 and Myc-Ophn1 or HA-Cdh1 and Myc-Ophn1 plasmids were subjected to immunoprecipitation with the HA antibody followed by immunoblotting with Myc antibody. D. Lysates of 293T transfected with control vector pKH3 and GFP-Cdh1, pEGFP and HA-p190GAP or GFP-Cdh1 and HA-p190GAP plasmids were subjected to immunoprecipitation with the GFP antibody followed by immunoblotting with the HA antibody. E. Lysates of $293 \mathrm{~T}$ transfected with control vector pCDNA3 and HA-Cdh1, pCMV5 and Mycp250GAP or HA-Cdh1 and Myc-p250GAP plasmids were subjected to immunoprecipitation with the HA antibody followed by immunoblotting with Myc antibody. 


\section{MADHUVANTHI KANNAN}

Place and date of birth: Chennai (India), 31.12.1984

\section{EDUCATION}

Georg-August-Universität, Germany

PhD Molecular and Cellular Neurobiology

2005-06

University of Leicester, $U K$

BSc Biochemistry

\section{RESEARCH EXPERIENCE}

PhD thesis: "The role of the E3 ubiquitin ligase Cdh1-APC in axon 2008growth in the mammalian brain", Dr. Judith Stegmüller, Max Planck Institute of Experimental Medicine, Göttingen, Germany

MSc thesis: "Studying the effect of mevastatin on the expression of ageing related genes in cortical neurons sufficient and depleted in heme", Dr. Tanya Chernova, MRC Toxicology Unit, Leicester, UK

\section{PUBLICATIONS}

Kannan, $\mathbf{M}$ et al. The E3 ligase Cdh1-APC operates upstream of the RhoA GTPase activating protein p250GAP in the control of axon growth in the mammalian brain. In preparation.

Kannan, $\mathbf{M}$ et al. (2012). The E3 ligase Cdh1-anaphase promoting complex operates upstream of the E3 ligase Smurf1 in the control of axon growth. Development. In press.

Kannan, M et al. (2010). Mevastatin accelerates loss of synaptic proteins and neurite degeneration in aging cortical neurons in a heme-independent manner. Neurobiol Aging 31(9), 1543-53.

\section{ACADEMIC ENCOMIA}

- Open University Scholarship for Masters Studies

2005-06

University of Leicester, UK

- KVPY National Science Fellowship for Undergraduate Research 